\title{
Effect of Mechanical Stimulation on Mesenchymal Stem Cell Seeded Cartilage Constructs
}

\author{
Karin Wartella \\ Virginia Commonwealth University
}

Follow this and additional works at: https://scholarscompass.vcu.edu/etd

Part of the Biomedical Engineering and Bioengineering Commons

(C) The Author

\section{Downloaded from}

https://scholarscompass.vcu.edu/etd/2231

This Dissertation is brought to you for free and open access by the Graduate School at VCU Scholars Compass. It has been accepted for inclusion in Theses and Dissertations by an authorized administrator of VCU Scholars Compass. For more information, please contact libcompass@vcu.edu. 
${ }^{\circ}$ Karin A Wartella, 2010

All Rights Reserved 
EFFECT OF MECHANICAL STIMULATION ON MESENCHYMAL STEM CELL SEEDED CARTILAGE CONSTRUCTS

A dissertation submitted in partial fulfillment of the requirements for the degree of Doctor of Philosophy, Biomedical Engineering at Virginia Commonwealth University.

By

KARIN ANASTASIA WARTELLA

M.S., Virginia Commonwealth University 2005

B.S., King's College, 1996

Director: JENNIFER S. WAYNE, PHD

PROFESSOR, BIOMEDICAL ENGINEERING

Virginia Commonwealth University

Richmond, Virginia

August, 2010 


\section{Acknowledgment}

I would like to thank several people for support with my dissertation project. First, I would like to express my sincere gratitude and appreciation to my advisor Dr Jennifer Wayne for all her patience, guidance, and direction with my research project. I would also like to thank my committee members: Drs D-Y. Fei, R. Rao, H.Yang, and W. Zuelzer for their support and participation in my dissertation project. A special thanks to my Mom, Dad, and Nana for their love, care, support, and encouragement. I would also like to especially thank my sister and best friend, Jennifer for all her love, support, and guidance with seeing me through to the end of my dissertation. I would also like to thank my extended family for their support and a special thanks to both the past and present members of the Orthopaedic Research Laboratory, Kelly, Johnny, Pete, Joseph, and Meade. I am particularly appreciative of all the help given by Aparna Maiti with Western Blot Analysis. Additionally, I would like to thank the Department of Biomedical Engineering, School of Engineering, and the Department of Orthopaedics, especially Drs. Hull, Cardea, and Jiranek for the bone marrow samples from hip replacement patience. I would also like to thank Kensey Nash Corporation for the endless supply of collagen type I sponge scaffolds. Thanks to my special running buddies Christy and Nicole for letting me "vent" during all our mileage. Finally, I would like especially to thank Scott Sturm for all his support and encouragement especially with PS. 
Table of Contents

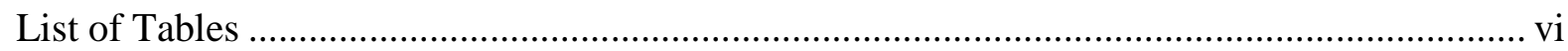

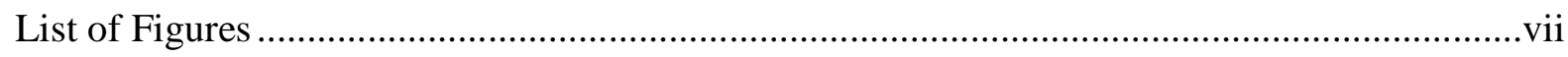

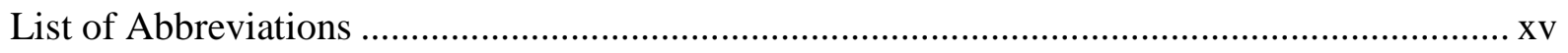

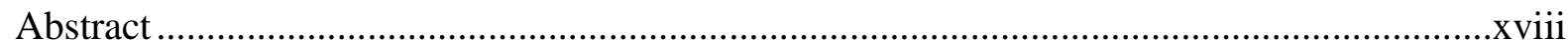

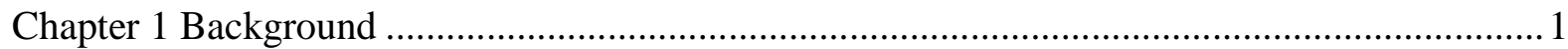

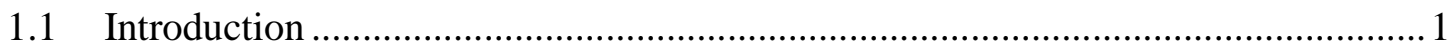

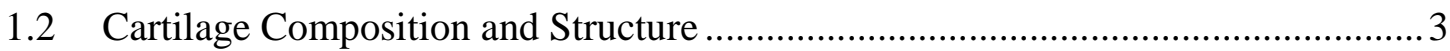

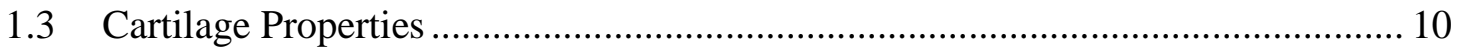

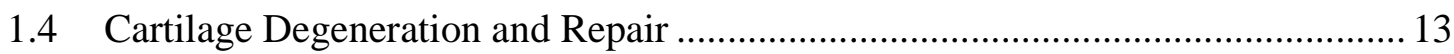

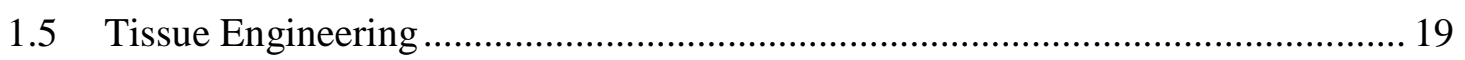

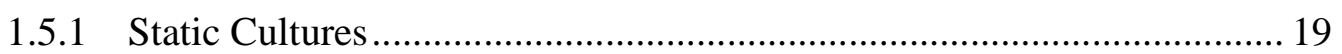

1.5.2 Application of Compression ......................................................... 23

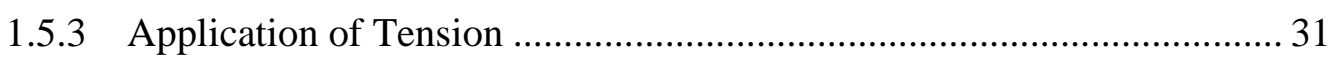

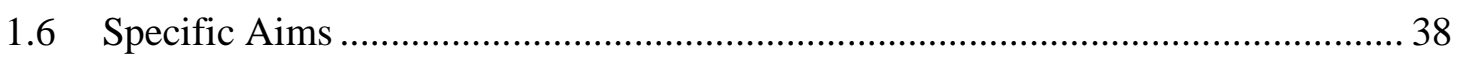

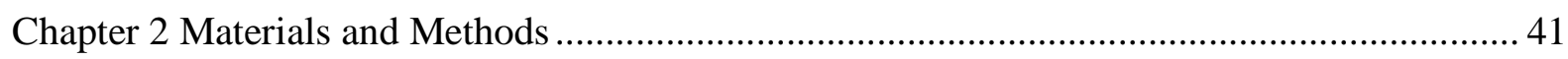

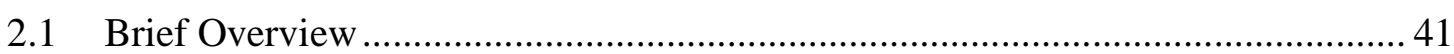

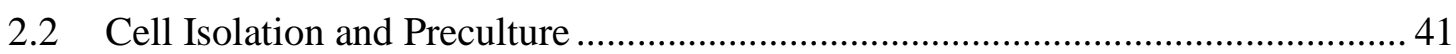

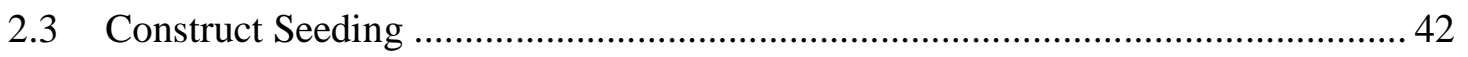

2.4 Optimal Seeding Density and Static Culture Time …......................................... 44

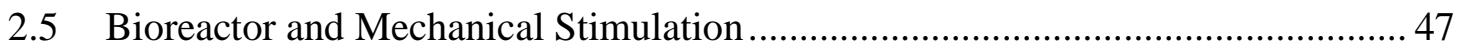

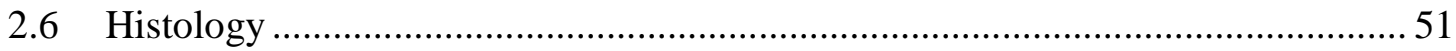

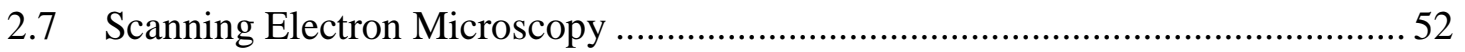

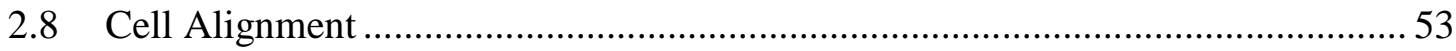

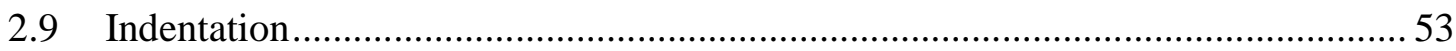

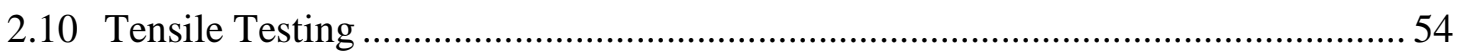

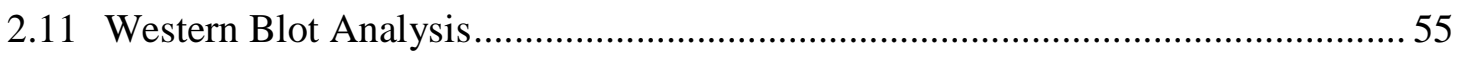




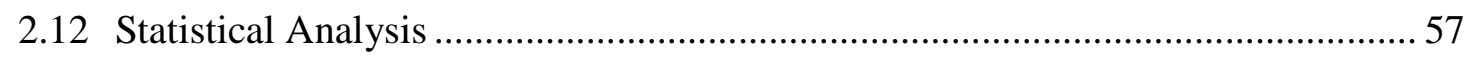

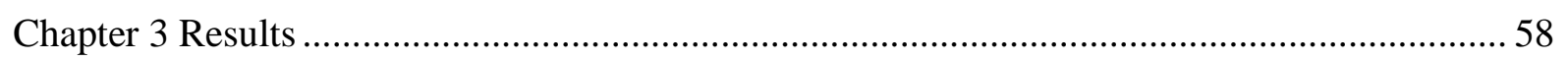

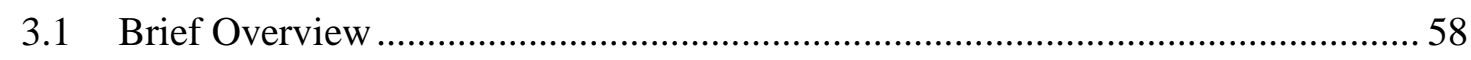

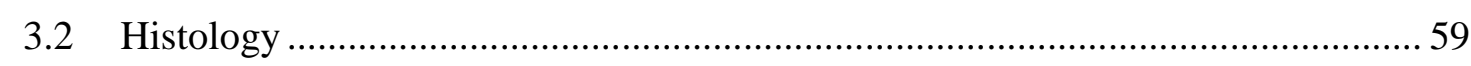

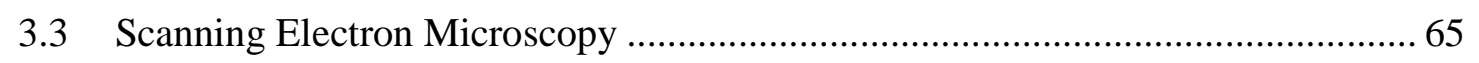

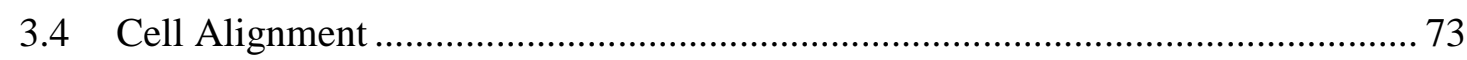

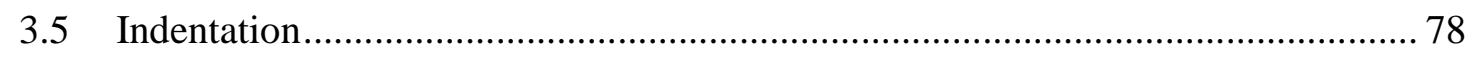

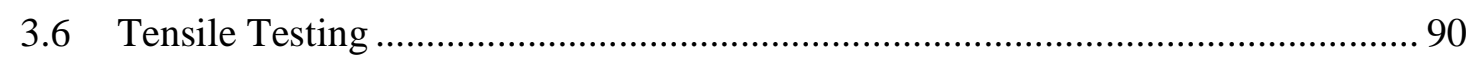

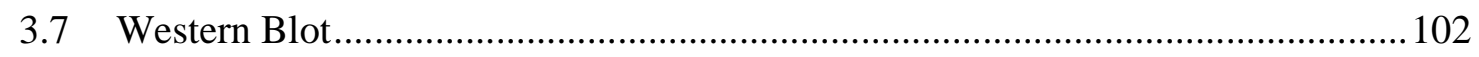

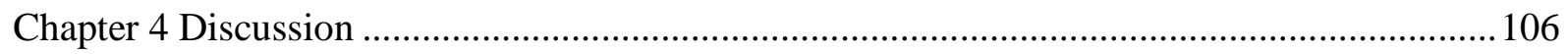

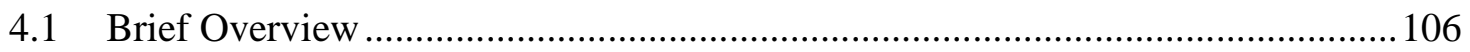

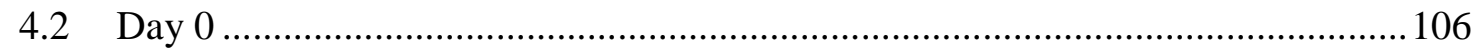

4.3 Compression and Tension Without Offset ........................................................ 111

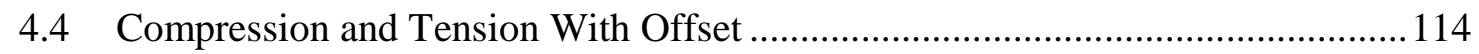

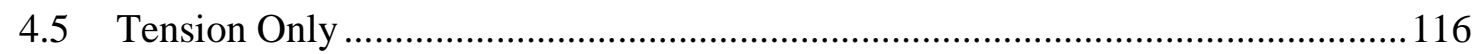

4.6 Conclusions, Limitations, and Future work ........................................................ 118

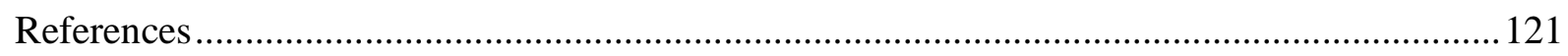

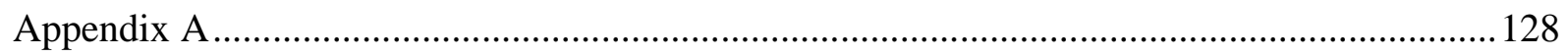

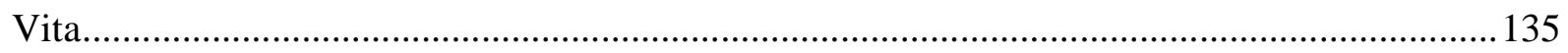


List of Tables

Table 3.1 Data summary for all constructs indentation tested............................................. 88

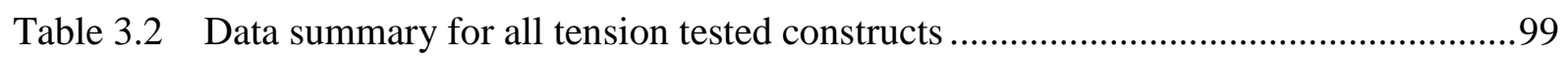


Figure 1.1 Image of alcian blue stained bovine articular cartilage illustrating the superficial tangential zone structure with regard to A) proteoglycans, B) chondrocyte cells, and C) collagen type II

Figure 1.2 Schematic of the Zones of Articular Cartilage depicting the Superficial Zone, Intermediate Zone, Deep Zone, and Calcified Zone. Cells in the Superficial Zone lay in a parallel pattern to the surface; the Intermediate Zone has chondrocytes in a more random pattern, and the Deep Zone the cells are in a columnar pattern.

Figure 1.3 Schematic illustrating the collagen fiber arrangement through the layers of cartilage tissue. The fiber in the Superficial Zone are arranged in a pattern parallel to the surface, in the Middle Zone they are more random, and in the Deep Zone they are perpendicular to the surface and insert through the tide mark into the calcified cartilage area

Figure 2.1 Photograph of Kensey Nash Collagen Type I scaffolding in sterile packaging ........ 42

Figure 2.2 Photograph of seeded constructs in chondrogenic media within a 6-well plate ........ 43

Figure 2.3 Schematic and image of the permeability testing apparatus 45

Figure 2.4 Permeability values for the 3 culture times, 2 seeding densities $\left(A=2 \times 10^{3}\right.$ and $B=4$ $\mathrm{x} 10^{3}$ cells $/ \mathrm{mm}^{3}$ ), and 3 pressures

Figure 2.5 Alcian blue histological pictures of constructs. A) Higher density at week 2, B) lower density at week $2, \mathrm{C}$ ) higher density at week 1 , and D) lower density at week 1 . $(10 \mathrm{X})$

Figure 2.6 Photograph of the bioreactor loaded with samples, placed within the incubator, and connected to the peristaltic pump

Figure 2.7 Schematic of the stimulation algorithm for stimulation 1 at $10 \%$ cyclic strain stimulation for both compression and tension.

Figure 2.8 Schematic of the stimulation algorithm for stimulation 2 at $10 \%$ total strain stimulation with 5\% offset and 5\% cyclic strain for both compression and tension........50

Figure 2.9 Schematic of the stimulation algorithm for stimulation 3 at $10 \%$ cyclic strain for tension only. 
Figure 3.1 Schematic diagram of the locations within constructs for histological and SEM sections: (A) external edge parallel to the tension direction, (B) external surface in the direction of tension, $(\mathrm{C})$ internal edge in the direction of tension parallel to the external edge, (D) internal plane section parallel to the surface in the direction of tension, and (E) internal edge perpendicular to the direction of tension (direction of compression is indicated with single-headed red arrow and tension is indicated with double-headed arrow).

Figure 3.2 Histology image of a Kensey Nash collagen type 1 scaffold of an internal edge section (A) and an interior plane parallel to surface section (B) (scale bar is $100 \mu \mathrm{m}$ ) ...59

Figure 3.3 Construct 155A (A and B) and Construct 186D (C and D), Day 0 with no mechanical stimulation. Shown are edge sections with black arrows indicating the exterior surface (A and $\mathrm{C}$ ) and a plane parallel to the surface (B and D). Cells are identified in the images with white circles and blue GAG staining with white arrows (scale bar is $100 \mu \mathrm{m}$ ).

Figure 3.4 Construct 163A (A and B) and Construct 209A (C and D) with both compression and tension without offset. Shown are edge sections, with black arrows indicating the exterior surface (A and C), and interior plane sections (B and D). Cells are identified in the images with white circles and blue GAG staining with white arrows (scale bar is 100 $\mu \mathrm{m})$.

Figure 3.5 Construct 186B (A and B) and Construct 188B (C and D) with both compression and tension with an offset, illustrating edge sections, with black arrows indicating the exterior surface (A and C) and interior plane sections (B and D). Cells are identified in the images with white circles and blue GAG staining with white arrows (scale bar is 100 $\mu \mathrm{m})$.

Figure 3.6 Construct 197B (A and B) and Construct 199A (C and D) with tension only, illustrating edge sections, with black arrows indicating the exterior surface (A and C) and interior plane sections (B and D). Cells are identified in the images with white circles and blue GAG staining with white arrows (scale bar is $100 \mu \mathrm{m}$ ).

Figure 3.7 Kensey Nash collagen type I scaffold (A) edge and (B) exterior surface section view (scale bar is $20 \mu \mathrm{m}$ )

Figure 3.8 Construct 212A with no stimulation, illustrating an (A) internal edge section view, (B) internal parallel to interior plane section, and (C) external surface section (scale bar is $20 \mu \mathrm{m})$. White circles indicate cells and a white arrow indicates matrix. .66

Figure 3.9 Construct 212B with no stimulation, illustrating an (A) internal edge section view, (B) internal parallel to interior plane section, and (C) external surface section (scale bar is $20 \mu \mathrm{m})$. White circles indicate cells and a white arrow indicates matrix. 
Figure 3.10 Construct 166C with both compression and tension without offset, illustrating an (A) internal edge section view, (B) internal parallel to interior plane section, and (C) internal edge section perpendicular to tension (tension direction is going in to picture). White circles indicate cells, white single-headed arrows indicate matrix, and white doubleheaded arrows indicate direction of tension application (scale bar is $20 \mu \mathrm{m}$ ).

Figure 3.11 Construct 170A with both compression and tension without offset, illustrating an (A) internal edge section view, (B) internal parallel to interior plane section, and (C) internal edge section perpendicular to tension (tension direction is going in to picture). White circles indicate cells, white single-headed arrows indicate matrix, and white doubleheaded arrows indicate direction of tension application (scale bar is $20 \mu \mathrm{m}$ ).

Figure 3.12 Construct 186B with both compression and tension with offset, illustrating an (A) internal edge section view, (B) internal parallel to interior plane section, and (C) internal edge section perpendicular to tension (tension direction is going in to picture). White circles indicate cells, white single-headed arrows indicate matrix, and white doubleheaded arrows indicate direction of tension application (scale bar is $20 \mu \mathrm{m}$ ). 70

Figure 3.13 Construct 188B with both compression and tension with offset, illustrating an (A) internal edge section view, (B) internal parallel to interior plane section, and (C) internal edge perpendicular to tension (tension direction is going in to picture). White circles indicate cells, white single-headed arrows indicate matrix, and white double-headed arrows indicate direction of tension application (scale bar is $20 \mu \mathrm{m}$ ).

Figure 3.14 Construct 198B with tension only, illustrating an (A) internal edge section, (B) internal parallel to interior plane section, and $(\mathrm{C})$ internal edge section perpendicular to tension (tension direction is going in to picture). White circles indicate cells, white single-headed arrows indicate matrix, and white double-headed arrows indicate direction of tension application (scale bar is $20 \mu \mathrm{m}$ ). .72

Figure 3.15 Construct 199A with tension only, illustrating an (A) internal edge section, (B) internal parallel to interior plane section, and $(\mathrm{C})$ internal edge section perpendicular to tension (tension direction is going in to picture). White circles indicate cells, white single-headed arrows indicate matrix, and white double-headed arrows indicate direction of tension application (scale bar is $20 \mu \mathrm{m}$ ).

Figure 3.16 Example analysis image of alignment completed on a compression and tension image of specimen $163 \mathrm{C}$ subjected to compression and tension with no offset (10X) ....74

Figure 3.17 Histogram of the frequency for cellular alignment in constructs with the respect to the direction of applied tension for all stimulation protocols. 
Figure 3.18 The relative frequency of the angle from the day 0 constructs with no stimulation .75

Figure 3.19 The relative frequency of the angle from the stimulated constructs with both compression and tension without offset. .75

Figure 3.20 The relative frequency of the angle from the stimulated constructs with both compression and tension and an offset. .76

Figure 3.21 The relative frequency of the angle from the stimulated constructs with tension only .77

Figure 3.22 Average cell angle for all stimulation protocols in the alignment analysis (+indicates $\mathrm{p}<0.001$ vs. Both no offset, Both with offset, and Tension only; *indicates $\mathrm{p}<0.001$ vs. Both with offset and Tension only) .78

Figure 3.23 Normalized creep response (displacement/construct thickness) from indentation testing of constructs (A) 156A, (B) 156B, and (C) 157C and the corresponding curve-fit

Figure 3.24 Normalized creep response (displacement/construct thickness) from indentation testing of construct (A) 157D, (B) 158E, and (C) $158 \mathrm{~F}$ and the corresponding curve-fit.

Figure 3.25 Normalized creep response (displacement/construct thickness) from indentation testing of construct (A) 207A, (B) 207B, and (C) 207C and the corresponding curve-fit.

Figure 3.26 Normalized creep response (displacement/construct thickness) from indentation testing of construct (A) 163A, (B) 163B, and (C) 163C and the corresponding curve-fit.

Figure 3.27 Normalized creep response (displacement/construct thickness) from indentation testing of construct (A) 167B, (B) 167C, and (C) 169C and the corresponding curve-fit.

Figure 3.28 Normalized creep response (displacement/construct thickness) from indentation testing of construct (A) 209A, (B) 209B, and (C) 209C and the corresponding curve-fit.

Figure 3.29 Normalized creep response (displacement/construct thickness) from indentation testing of construct (A) 184A, (B) 184B, and (C) 184C and the corresponding curve-fit. 
Figure 3.30 Normalized creep response (displacement/construct thickness) from indentation testing of construct (A) 186A, (B) 186C, and (C) 188A and the corresponding curve-fit.

Figure 3.31 Normalized creep response (displacement/construct thickness) from indentation testing of construct (A) 205B, (B) 205C, and (C) 205D and the corresponding curve-fit.

Figure 3.32 Normalized creep response (displacement/construct thickness) from indentation testing of construct (A) 178A, (B) 178C, and (C) 181A and the corresponding curve-fit.

Figure 3.33 Normalized creep response (displacement/construct thickness) from indentation testing of construct (A) 197A, (B) 197B, and (C) 198B and the corresponding curve-fit.

Figure 3.34 Normalized creep response (displacement/construct thickness) from indentation testing of construct (A) 208A, (B) 208B, and (C) 208C and the corresponding curve-fit.

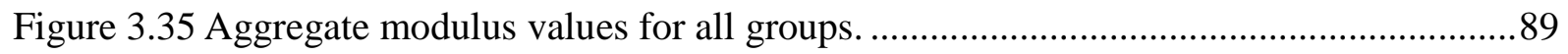

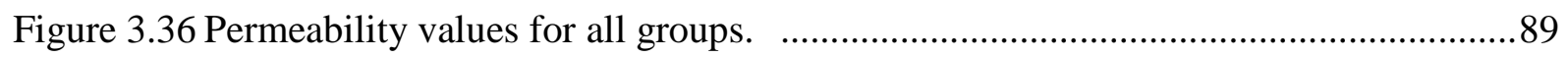

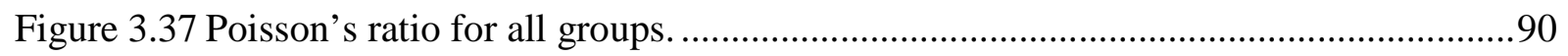

Figure 3.38 Stress versus strain curves for constructs (A) 156A, (B) 156B, (C) 157C, (D) 157D, and (E) 158E; the black line indicates the region used for calculation of modulus..........91

Figure 3.39 Stress versus strain curves for constructs (A) 158F, (B) 207A, (C) 207B, and (D) 207C; the black line indicates the region used for calculation of modulus

Figure 3.40 Stress versus strain curves for constructs (A) 163A, (B) 163B, (C) 163C, (D) 167B, and (E) $167 \mathrm{C}$; the black line indicates the region used for calculation of modulus

Figure 3.41 Stress versus strain curves for constructs (A) 169C, (B) 209A, (C) 209B, and (D) 209C; the black line indicates the region used for calculation of modulus

Figure 3.42 Stress versus strain curves for constructs (A) 184A, (B) 184B, (C) 184C, (D) 186A, and $186(\mathrm{C})$; the black line indicates the region used for calculation of modulus. .95

Figure 3.43 Stress versus strain curves for constructs (A) 188A, (B) 205B, (C) 205C, and (D) 205D; the black line indicates the region used for calculation of modulus .96 
Figure 3.44 Stress versus strain curves for constructs (A) 178A, (B) 178C, (C) 181A, (D) 197A, and (E) 197B; the black line indicates the region used for calculation of modulus

Figure 3.45 Stress versus strain curves for constructs (A) 198A (B) 208A, (C) 208B, and (D) $208 \mathrm{C}$; the black line indicates the region used for calculation of modulus .98

Figure 3.46 Cross-sectional area for each of the groups......................................................100

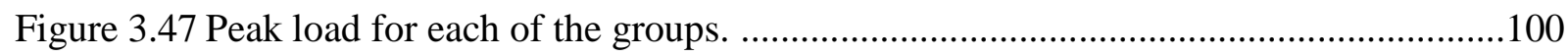

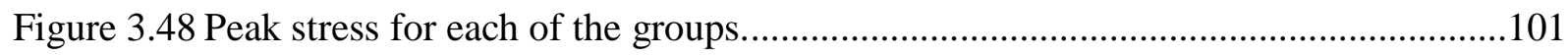

Figure 3.49 Tensile modulus for each of the tested groups. ....................................................101

Figure 3.50 Picture of western blot gels after treatment with antibody to aggrecan was completed A) major band of aggrecan, B) minor band of aggrecan, and C) GAPDH (loading control). 102

Figure 3.51 Relative protein intensity for aggrecan from the upper band for human articular cartilage and all tested groups normalized to the GAPDH band. 103

Figure 3.52 Relative protein intensity for aggrecan from the lower band for human articular cartilage and all tested groups normalized to the GAPDH band 104

Figure A.1 Construct 155A (A and B) and Construct 186D (C and D), Day 0 with no mechanical stimulation. Shown are edge sections with black arrows indicating the exterior surface (A and C) and a plane parallel to the surface (B and D). (scale bar is $100 \mu \mathrm{m}$ ).

Figure A.2 Construct 155A (A and B) and Construct 186D (C and D), Day 0 with no mechanical stimulation. Shown are edge sections with black arrows indicating the exterior surface (A and C) and a plane parallel to the surface (B and D). (scale bar is $100 \mu \mathrm{m}$ )

Figure A.3 Construct 163A (A and B) and Construct 209A (C and D) with both compression and tension without offset. Shown are edge sections, with black arrows indicating the exterior surface (A and C), and interior plane sections (B and D). (scale bar is $100 \mu \mathrm{m}$ ).

Figure A.4 Construct 163A (A and B) and Construct 209A (C and D) with both compression and tension without offset. Shown are edge sections, with black arrows indicating the exterior surface (A and C), and interior plane sections (B and D). (scale bar is $100 \mu \mathrm{m}$ ). 
Figure A.5 Construct 186B (A and B) and Construct 188B (C and D) with both compression and tension with an offset, illustrating edge sections, with black arrows indicating the exterior surface (A and C) and interior plane sections (B and D). (scale bar is $100 \mu \mathrm{m}$ )

Figure A.6 Construct 186B (A and B) and Construct 188B (C and D) with both compression and tension with an offset, illustrating edge sections, with black arrows indicating the exterior surface (A and C) and interior plane sections (B and D). (scale bar is $100 \mu \mathrm{m}$ )

Figure A.7 Construct 197B (A and B) and Construct 199A (C and D) with both compression and tension with an offset, illustrating edge sections, with black arrows indicating the exterior surface (A and C) and interior plane sections (B and D). (scale bar is $100 \mu \mathrm{m}$ ) 130

Figure A.8 Construct 197B (A and B) and Construct 199A (C and D) with both compression and tension with an offset, illustrating edge sections, with black arrows indicating the exterior surface (A and C) and interior plane sections (B and D). (scale bar is $100 \mu \mathrm{m}$ ) 130

Figure A.9 Construct 212A with no stimulation, illustrating an (A) internal edge section view and (B) internal parallel to interior plane section (scale bar is $20 \mu \mathrm{m}$ )

Figure A.10 Construct 212B with no stimulation, illustrating an (A) internal edge section view and (B) internal parallel to interior plane section (scale bar is $20 \mu \mathrm{m}$ )

Figure A.11 Construct 166C with both compression and tension without offset, illustrating an (A) internal edge section view and (B) internal parallel to interior plane section, white double-headed arrows indicate direction of tension application (scale bar is $20 \mu \mathrm{m}$ ).....132

Figure A.12 Construct 170A with both compression and tension without offset, illustrating an (A) internal edge section view and (B) internal parallel to interior plane section, white double-headed arrows indicate direction of tension application (scale bar is $20 \mu \mathrm{m}$ ).....132

Figure A.13 Construct 186B with both compression and tension with offset, illustrating an (A) internal edge section view and (B) internal parallel to interior plane section, white double-headed arrows indicate direction of tension application (scale bar is $20 \mu \mathrm{m}$ ).....132

Figure A.14 Construct 188B with both compression and tension with offset, illustrating an (A) internal edge section view and (B) internal parallel to interior plane section, white double-headed arrows indicate direction of tension application (scale bar is $20 \mu \mathrm{m}$ ).....132

Figure A.15 Construct 198B with tension only, illustrating an (A) internal edge section view and (B) internal parallel to interior plane section, white double-headed arrows indicate direction of tension application (scale bar is $20 \mu \mathrm{m}$ ). 
Figure A.16 Construct 198B with tension only, illustrating an (A) internal edge section view and (B) internal parallel to interior plane section, white double-headed arrows indicate direction of tension application (scale bar is $20 \mu \mathrm{m}$ ). 


\section{LIST OF ABBREVIATIONS}

degree

3-D

three-dimensional

ANOVA

analysis of variance

BMP

bone morphogenetic protein

${ }^{0} \mathrm{C}$

degrees Celsius

cAMP

cyclic adenosine 3'-5' monophosphate

$\mathrm{CO}_{2}$

carbon dioxide

CDC

Center for Disease Control

DNA

deoxyribonucleic acid

DC

direct current

DMEM

Dulbecco's Modified Eagles Medium

E

Young's modulus

ECM

extracellular matrix

FBS

fetal bovine serum

FGF-2

fibroblast growth factor 2

GAG

glycosaminoglycan

GAPDH

Glyceraldehyde-3-phosphate dehydrogenase

$\mathrm{H}_{\mathrm{A}}$

aggregate modulus

$\mathrm{H} \& \mathrm{E}$

hematoxylin and eosin 
$\mathrm{Hz}$

IGF-I or II

$\mathrm{k}$

$\mathrm{kDa}$

$\mathrm{kgfcm}^{-2}$

$\mathrm{kPa}$

LVDT

MMP

MSCs

$\mathrm{MPa}$

$\mathrm{N}$

$\mathrm{Nu}$

$\mathrm{OA}$

PBS

PBT

PEGT

PG(s)

RT-PCR

$\sigma$

$\varepsilon$

SA

SEM hertz

Insulin-like growth factor-I or II

permeability

kilodaltons

kilogram force/centimeters squared

kilopascals

linear variable differential transducer

metalloproteinases

mesenchymal stem cells

megapascals

Newtons

Poisson's Ratio

osteoarthritis

phosphate buffered saline

poly(butylenes teraphathalate)

poly(ethylene glycol teraphathalate)

proteoglycan(s)

reverse transcription polymerase chain reaction

stress

strain

stretch activated

scanning electron microscopy 
Stdev

STZ

TIMP

TGF- $\beta$ standard deviation

superficial tangential zone

tissue inhibitor metalloproteinases

transforming growth factor-beta 


\section{EFFECT OF MECHANICAL STIMULATION ON MESENCHYMAL STEM CELL SEEDED CARTILAGE CONSTRUCTS}

\section{BY KARIN ANASTASIA WARTELLA, M.S.}

A dissertation submitted in partial fulfillment of the requirements for the degree of Doctor of Philosophy at Virginia Commonwealth University.

Virginia Commonwealth University, 2010

Major Director: Jennifer S. Wayne, PhD

Professor, Biomedical Engineering

Due to the high incidence of injury and osteoarthritis and the limited nature of articular cartilage self-repair, alternate means are necessary to replace the damaged tissue and regain an articulating surface that not only stops the progression of disease but functions the same as a healthy articular surface. This study focus on the early stages of damage, particularly when the outer surface layer, the superficial tangential zone, becomes damaged and fibrillation begins. We hope to achieve this by creating a tissue replacement product through tissue engineering using an abundant cell source of mesenchymal stems cells seeded in Collagen Type I scaffolds, and treated not only with chondrogenic growth media in culture, but three different stimulation algorithms to improve structural and functional properties.

The cell source was obtained from bone marrow specimens from healthy human subjects undergoing hip replacement surgery. Cells were expanded, seeded on collagen type I scaffolds and grown statically for 1 week. Following 1 week, constructs were either analyzed for histology, SEM, alignment, indentation, tensile, and aggrecan content with Western Blot analysis 
or stimulated for 2 additional weeks. Each of the following algorithms comprises one of the three stimulation groups, which were chosen to produce characteristics similar to the superficial tangential zone of articular cartilage: application of compression and tension without offset, application of compression and tension with offset, and tension alone.

Results showed GAG staining from histology in all test groups, however there was more abundance in the stimulated groups. For SEM results there appeared more matrix components and organization in the stimulation groups with both compression and tension. Evaluation of cell alignment showed the test group with both compression and tension without offset trending toward alignment with the direction of applied tension indicating the stimulation algorithm was producing strain effects to cause the cells to preferentially align. Additionally, this group had the best mechanical property outcome. Western blot results showed the group with compression and tension without offset had similar aggrecan content to native articular cartilage results, as well as the other stimulation groups, indicating stimulation aids in producing aggrecan content similar to the superficial tangential zone.

The overall results showed the group stimulated with both compression and tension without offset had the best structure and functional properties of the group. Although the mechanical properties were inferior to native articular cartilage, they were within range of repair tissue. Further exploration with both compression and tension without offset stimulation in longer culturing can enhance these aspects and lead to prevention of further cartilage degradation in patients with early signs of osteoarthritis or damaged articulating surface. 


\section{CHAPTER 1 BACKGROUND}

\subsection{INTRODUCTION}

It is estimated in the US alone that increasing medical care costs and loss of productivity is up to $\$ 300$ billion for disability related problems in adults. The CDC and US Census Bureau list arthritis and rheumatism as the most prevalent cause of disabilities accounting for $19 \%$ of the total; which will likely increase with the growing population of adults over the age $65^{1}$. Osteoarthritis (OA) is the most common type of arthritic diseases causing major disability usually starting with stiffness in the joint and a gradual onset of pain ${ }^{2}$; clinical characteristics of pain, effusion, gait problems, and loss of articular cartilage, with subchondral bone remodeling lead to an OA diagnosis ${ }^{3}$. Causes of OA can be from a multitude of sources such as genetic defects, overuse leading to injury, metabolic diseases (diabetes, excess weight), malformation of joint, and trauma, to name a few ${ }^{3}$.

Osteoarthritis involves a disruption of the hyaline articular surface coinciding with damage to the underlying subchondral bone ${ }^{4}$. Articular cartilage is a specialized connective tissue covering all moveable joints within the body, with no blood vessels, nerves, or connection to the lymphatic system ${ }^{5}$. Undamaged cartilage provides a smooth, almost frictionless surface that distributes the load of articulation across the joint, minimizing the stresses exerted on the underlying bone, keeping its integrity for decades of wear even under high repeatable stresses ${ }^{6}$. However, once damage does occur, the composition and structure of cartilage as well as the environment of the joint do not allow for a repair mechanism that restores the properties of the original articulating surface.

Currently, restoration of the articulating surface to reduce pain involves one of a multitude of therapeutic strategies. These methods range from techniques that require modest 
adjustment to the damaged region to complete replacement of the damaged joint. Some modest methods are arthroscopy, shaving, debridement, and drilling into underlying bone, to name of few. One of the up and coming areas is to repair the surface with a functional tissue engineered replacement ${ }^{7}$. The surface layer, composed of two layers, is covered in lamina splendens, a thin clear film that can be mechanically stripped from underlying cartilage and is composed of a cellfree amorphous layer, proteoglycan monomers and link protein on or in association with lower layer composed of cells, collagens, and proteoglycans ${ }^{5,8}$. Removal of the surface layer, known as the superficial tangential zone (STZ) increases the permeability of the tissue and probably increases loading of the macro molecular framework during compression. Disruption or remodeling of the dense collagenous matrix in the STZ is the earliest detectable structural changes in experimentally induced degeneration of articular cartilage ${ }^{5}$. The focus of this research is to create a tissue replacement product similar in appearance and properties to the superficial tangential zone of articular cartilage (Figure 1.1), which includes the major extracellular matrix components of: proteoglycans macromolecules, chondrocyte cells aligned parallel to the surface, and a dense network of collagen type II aligned parallel to the surface. 


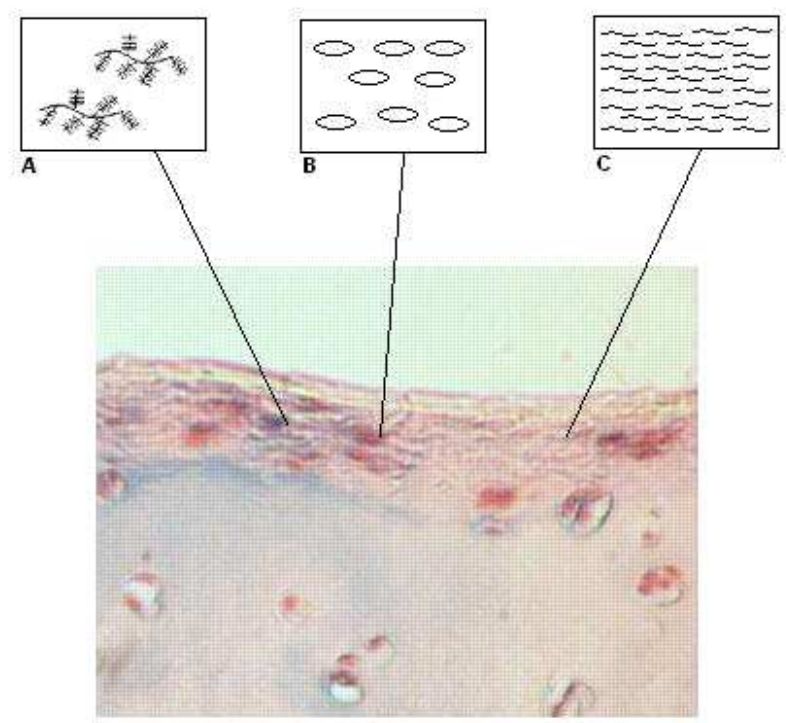

Figure 1.1 Image of alcian blue stained bovine articular cartilage illustrating the superficial tangential zone structure with regard to A) proteoglycans, B) chondrocyte cells, and C) collagen type II.

\subsection{CARTILAGE COMPOSITION AND STRUCTURE}

\section{Components of Cartilage}

Cartilage tissue is part of the connective tissue family composed of cells, interstitial fluid, and a matrix macromolecular support. The cells contribute a small amount, approximately $1 \%$ to the total volume of cartilage and similar to other tissues in its family, its mechanical properties are derived from the matrix. There is only one cell type residing within the tissue, chondrocytes, a highly specialized cell that varies within the different areas with regard to size, shape, and most likely metabolic activity ${ }^{8}$. These cells are responsible for the synthesis of the matrix and may recognize changes of the mechanics of the matrix. These cells do not form contacts with each other as many other cell types do; they surround themselves with the matrix molecular components. Chondrocytes usually are spheroidal in appearance and are the producers of Collagen type II, proteoglycans (PGs), and non-collagenous proteins. The continued activity of the cells within cartilage suggests the tissue is constantly undergoing remodeling, with both 
synthesis of the framework molecules and enzymes for degradation of the matrix ${ }^{8}$. Chondrocytes respond to their environmental changes in mechanical loading, growth factors, and interleukins ${ }^{9}$. With age, it is thought the ability of chondrocytes to maintain the extracellular matrix (ECM) declines, leading to degradation of the tissue ${ }^{8}$.

ECM is composed of both tissue fluid and structural support, which gives cartilage its structure and strength ${ }^{8}$. Water comprises $65-80 \%$ of the weight of healthy cartilage tissue with collagens and PGs macromolecules bearing the majority of the loading forces. Some of the water is allowed to move in and out of the tissue freely, which contains dissolved salts of sodium, potassium, calcium, and chloride. Water flow in and out of cartilage acts as a nutrient exchange for chondrocytes ${ }^{9}$. The interactions of the water and its components influence how cartilage tissue reacts when a mechanical force is applied. There are gases, small proteins, metabolites, and a large concentration of cations within the tissue fluid, which act to balance the proteoglycan negative charges, although the tissue is always in a state of imbalance. The Donnan effect is a result of tissue swelling from the increase in the osmolarity of the tissue, due to the negative charges associated with the proteoglycans. The inorganic ions associated with the PGs create the Donnan osmotic pressure by an imbalance between the interstitium and the external bathing solution, which causes the tissue to swell. At equilibrium, tensile forces of the collagen network balance the swelling pressure; therefore the tissue is always in a state of prestress ${ }^{10}$.

Cartilage tissue contains a $20-40 \%$ wet weight of collagens, proteoglycans, and noncollagenous proteins. Individually, collagens, proteoglycans, and noncollagenous proteins contribute to the dry weight of cartilage $60 \%, 25-35 \%$, and $15-20 \%$, respectively. The distribution of collagen fibers is uniform throughout the tissue, except for the collagen dense 
superficial zone. Collagen type II is the predominant type found within cartilage (90-95\%), forming the primary components of the ECM framework, with the remaining types of V, VI, IX, $\mathrm{X}$, and $\mathrm{XI}$ as minor contributions ${ }^{8,9}$. It is thought that Collagen type $\mathrm{X}$, which is usually located near the chondrocytes of the calcified zone, plays a role in calcification of cartilage ${ }^{8}$. Collagen type VI is found surrounding the chondrocytes in cartilage, which plays an important role in chondrocyte attachment to the matrix ${ }^{8}$, however, it does not seem to play a part in fiber formation, since it is easily isolated from tissue without enzymatic degradation ${ }^{11}$. Collagen types V and XI are believed to aid in regulation of fiber size; type IX may function as a spacer between fibrils, glue, or for interacting with proteoglycan molecules ${ }^{11}$.

Proteoglycan (PG) macromolecules are composed of a central core protein with one or more covalently bound carbohydrate side chains called glycosaminoglycans (GAGs) ${ }^{8,12}$. The GAG side chains are an assembly of unbranched long chains of polysaccharide with repeating disaccharides with an amino sugar. There is a negative charged carboxylate or sulfate group on each of the disaccharide units resulting in sequences of negative charges that serve to attract cations and repel other negative molecules. Hyaluronic acid, chondroitin sulfate, keratan sulfate, and dermatan sulfate are the types of GAGs found in cartilage tissue ${ }^{8}$. There are two main types of PGs classified as either aggregating or non-aggregating PGs. The largest aggregating PG, aggrecan, has many chains of chondroitin sulfate and keratan sulfate, which interacts with hyaluronic acid to create large PG complexes. This highly stable molecule provides a significant attraction for water molecules to hydrate the cartilage matrix ${ }^{12}$. The smaller non-aggregating PGs called decorin and fibromodulin may assist in organization and stabilization of Collagen type II framework. 
The remaining noncollagenous proteins and glycoproteins are not as well discerned as the collagens and PGs. These molecules are usually a protein core with a few connected monosaccharides or oligosaccharides. Their function appears to organize and maintain the matrix meshwork. Two that aid with chondrocyte binding are anchorin CII, binding chondrocytes to collagen fibrils, and cartilage oligomeric protein, which may aid with cartilage matrix synthesis. The non-collagenous proteins fibronectin and tenascin may have a role in matrix organization, cell-matrix interactions, and response to arthritis and OA, however, their function is still poorly understood ${ }^{8}$.

\section{Cartilage Zones and Regions}

The highly ordered organization of collagens, proteoglycans, and other molecules within articular cartilage is created by the chondrocytes. The composition, organization, and mechanical properties of the matrix along with the cell function vary with distance from the articulating surface. Additionally, there is a difference in composition, organization, and function of the matrix with regard to cell location ${ }^{8}$. Cartilage is considered composed of four distinct layers or zones between the articular surface to the subchondral bone, they are: the surface or superficial tangential zone, middle or transitional zone, the deep or radial zone, and calcified zones (Figure 1$)^{5}$. 


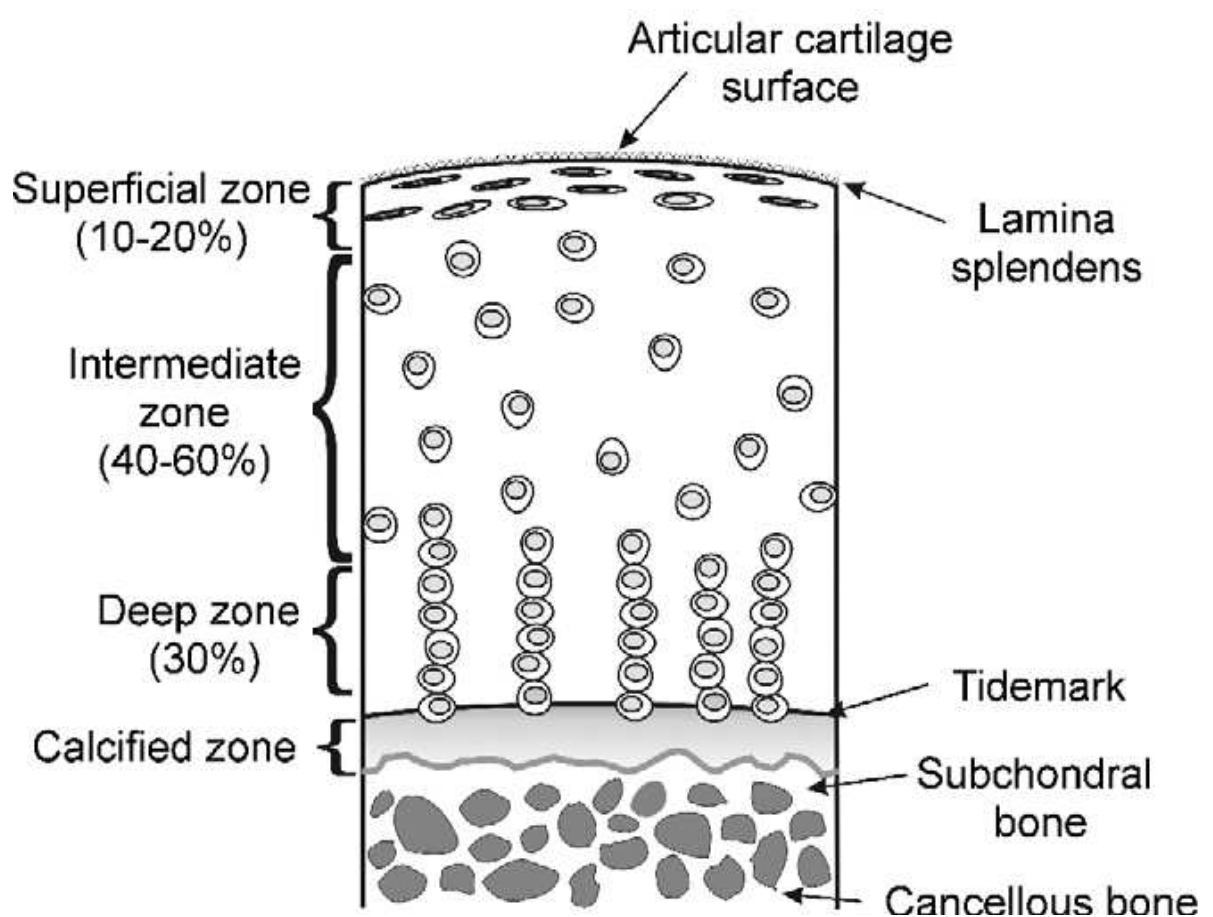

Figure 1.2 Schematic of the Zones of Articular Cartilage depicting the Superficial Zone, Intermediate Zone, Deep Zone, and Calcified Zone. Cells in the Superficial Zone lay in a parallel pattern to the surface; the Intermediate Zone has chondrocytes in a more random pattern, and the Deep Zone the cells are in a columnar pattern ${ }^{5}$.

These zones differ with their amounts of water, proteoglycan, and collagen; additionally, the aggrecan sizes vary between the layers. Furthermore, organization of the collagen network and chondrocyte size, shape, orientation differ between the zones ${ }^{8}$. 


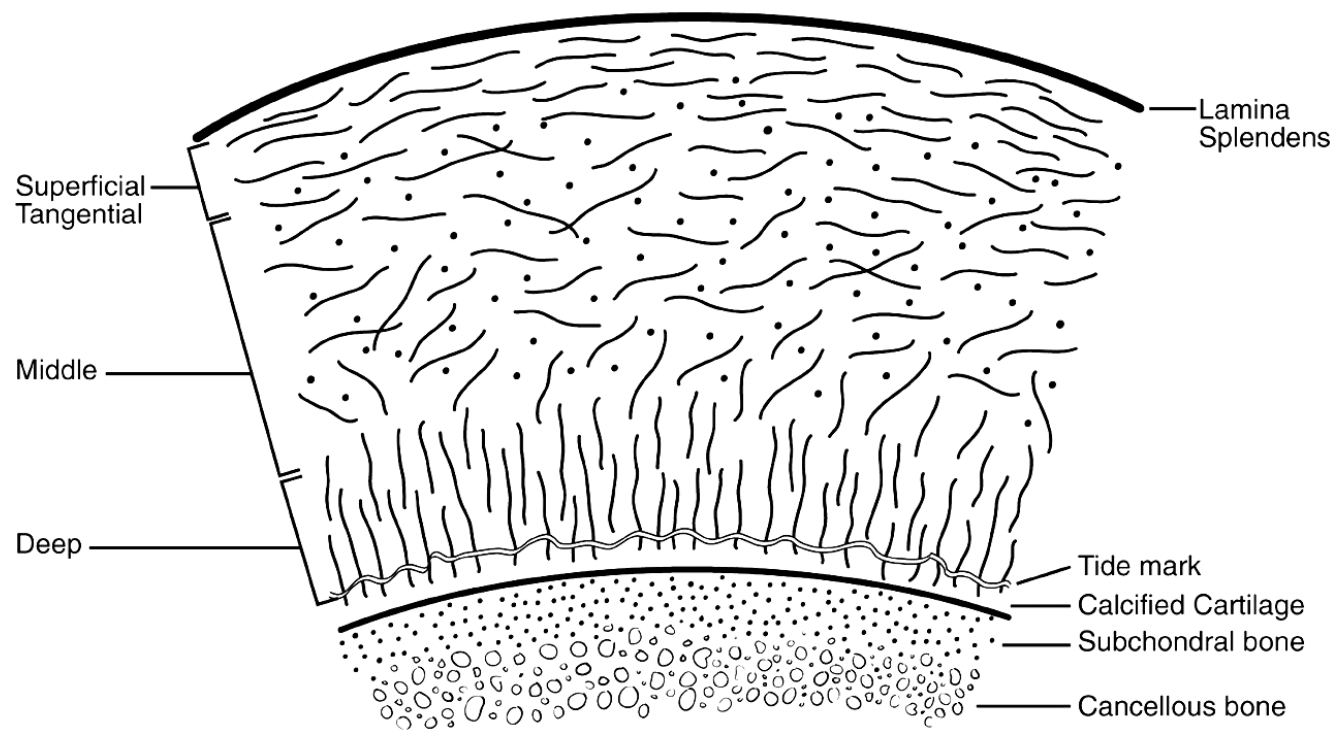

Figure 1.3 Schematic illustrating the collagen fiber arrangement through the layers of cartilage tissue. The fiber in the Superficial Zone are arranged in a pattern parallel to the surface, in the Middle Zone they are more random, and in the Deep Zone they are perpendicular to the surface and insert through the tide mark into the calcified cartilage area.

The STZ is located on the outermost layer of articular cartilage, which is the thinnest zone and covered by a layer of lamina splendens ${ }^{8,9}$. The lamina splendens is a sheet of fibrils with little polysaccharide and no cells, corresponding to a clear film and is believed to have a role in low friction lubrication ${ }^{8,13}$. The superficial zone's composition and ultrastructural arrangement gives it the unique mechanical and biologic properties. This zone contains ellipsoid-shaped cells arranged parallel to the articulating surface. These chondrocytes produce the high concentration of collagen matrix and the low concentration of proteoglycan with respect to the remaining zones. The collagen fibers of the superficial zone are a densely arranged weave parallel to the articular surface that aids in the mechanical strength (giving a higher tensile stiffness to this layer), maintains fluid pressurization within the tissue, and controls passage of nutrients and waste in and out of the cartilage tissue. Since this zone acts as a barrier to large molecules, when the layer is damaged, an inflammatory response may further damage the underlying tissue with inflammatory molecules achieving access to deeper cartilage zones ${ }^{8}$. 
The transitional zone, situated directly below the superficial tangential zone, contains fibers of collagen with a greater diameter laid out in a more random pattern, which the name implies, transitions between the superficial and deep zones. The chondrocytes have a more spheroid shape in appearance and seem more metabolically active in the transitional layer, since they have more organelles, endoplasmic recticulum, and Golgi membranes ${ }^{8,9}$. The volume of the transitional zone is much larger, with a higher concentration of proteoglycans and a lower concentration of both water and collagen than the superficial zone.

The deep zone contains collagen fibrils that are the largest in diameter within articular cartilage, with their orientation as perpendicular to the surface. Within this layer the chondrocytes appear spheroidal in shape and are arranged in a columnar pattern. The concentration of proteoglycans within this zone is at the highest in cartilage with the water content at the lowest concentration. The collagen fibers pass from this layer into the tidemark area, which corresponds to the boundary between the calcified and uncalcified cartilage ${ }^{8}$.

Below the deep zone is the calcified cartilage zone, which separates the uncalcified cartilage from the underlying bone ${ }^{8}$. The layer is thought to block the exchange of nutrients between articular cartilage and the bone, limiting cartilage to receive all its nutrition from the synovial fluid. In this zone the cells have a much smaller volume and contain low amounts of endoplasmic recticulum and Golgi membranes. Some regions have the cells completely surrounded by the calcified cartilage, which implies a very low metabolic activity in these cells, but they may play a role in development of osteoarthritis ${ }^{8}$.

Within a given zone, there are three distinct regions within articular cartilage tissue, a pericellular, a territorial, and an interterritorial region. The pericellular and territorial regions assist in binding the chondrocyte membrane to the matrix molecules, protecting the cells during 
loading and deformation. There is little or no fibril collagen in the pericellular region, it is rich in proteoglycans and non-collagenous proteins, and forms a thin layer completely around chondrocyte membrane ${ }^{8,9}$. Chondrocytes in this region have extensions of their cytoplasm that reach through the pericellular into the territorial region.

Territorial matrix surrounds the pericellular matrix of single chondrocytes, but in some locations it surrounds pairs or groups. Within the deep zone of cartilage the territorial region will surround the entire columns of chondrocytes. The majority of mature articular cartilage volume contains the interterritorial matrix, which contains the larger diameter collagen fibrils. This region is not organized to surround the chondrocytes, but progress from the surface in a parallel, to a random, and progress down to a perpendicular orientation in the deep zone ${ }^{8}$. The interterritorial zone is responsible for much of the biomechanical properties of articular cartilage that differ between the various zones ${ }^{9}$.

\subsection{CARTILAGE PROPERTIES}

\section{Cartilage Mechanical Behavior and Modeling}

Compression of cartilage tissue compacts the proteoglycan negative charges closer together and increases the repelling strength, which adds to the compressive stiffness. The mechanical behavior of cartilage forces is dependent on fluid flow through the collagen/PG network. Modeling cartilage as a combination of both fluid and solid components is referred to as a biphasic model of articular cartilage tissue. All solid-like components, proteoglycans, collagen, cells, and lipids comprise the solid phase, with the fluid comprising the interstitial solution that moves through the matrix. The solid phase is modeled as an incompressible elastic material with the fluid phase modeled as incompressible and inviscid. During instantaneous 
loading, the behavior of cartilage illustrates an incompressible, elastic solid without sufficient time for fluid to be released ${ }^{14}$. Maintenance of the applied load results in fluid flow within and out of the tissue causing tissue deformation.

\section{Cartilage Mechanical Properties}

Often a confined compression test is used to determine material properties of articular cartilage such as tissue creep and stress relaxation. In creep, a constant load is applied through a porous platen and the tissue is allowed to displace as a measure of time. Since the fluid cannot escape the matrix instantaneously, it requires time to come to equilibrium. In cartilage, the initial displacement is rapid with a large flow of fluid escaping the tissue, which is followed by a slowing in the rate of displacement and the flow of fluid out of the tissue. When equilibrium is achieved, there is a constant displacement and flow of fluid has ceased. Stress relaxation involves applying a constant deformation while the force is measured that is needed to maintain the tissue displacement ${ }^{14}$.

The confined compression creep test fitted to the biphasic model allows for determination of two material properties, the aggregate modulus and the tissue permeability. The measure of stiffness when fluid flow has ended is considered the aggregate modulus value and the resistance to fluid flow through the tissue matrix is the permeability. For normal articular cartilage tissue, the aggregate modulus is typically in the range of 0.5 to $0.9 \mathrm{MPa}$ and permeability is commonly in the range of $10^{-15}$ to $10^{-16} \mathrm{~m}^{4} / \mathrm{Ns}{ }^{15}$. Values of permeability are not constant throughout the tissue, with the superficial tangential zone having the highest value for permeability, allowing for

fluid flow at the surface and the deep zone exhibiting the lowest value ${ }^{16-18}$. Additionally, as cartilage tissue is compressed the permeability value decreases, thus most fluid comes from the surface region of cartilage and with heavier loads there is less fluid flow ${ }^{19,20}$. 
Tissue indentation testing is another valuable test for evaluating the mechanical properties of articular cartilage. Additionally, this test can be performed in situ allowing the cartilage tissue to stay attached to the subchondral bone. The other benefit of this test is it determines three independent material properties using the indenter when the biphasic model is applied to the data. The values determined are the aggregate modulus, Poisson's ratio, and permeability. Poisson's ratio is usually less than 0.4 , often approaching zero, illustrating cartilage as not completely incompressible material as once thought due to the fluid, which confirms the model of both a interstitial fluid phase and a solid matrix phase ${ }^{14}$.

Cartilage mechanical properties have also been evaluated for both its shear and tensile characteristics. For shear testing, application of small torsional loads to cartilage cylindrical plugs are often used to evaluate the tissue response. Results showed no real change of volume to cartilage to allow flow of fluid and since the fluid is mostly water with a very low viscosity, there is little resistance to shear forces ${ }^{21}$. Tensile testing of articular cartilage helps to illustrate the properties of anisotropy, inhomogeneity, and age-related changes. Tests are performed by thinly slicing the cartilage into sheets, once it is removed from the underlying bone. The slices are cut parallel to the articulating surface with a microtome and punched into a dumbbell shape. Samples from an orientation parallel to the split lines exhibit a higher tensile stiffness than those in a perpendicular direction. In skeletally mature individuals, there is a decrease in both tensile strength and stiffness from the superficial tangential zone to the lower zones, which is exactly opposite that in skeletally immature cartilage ${ }^{22}$.

Articular cartilage is considered viscoelastic, due to its time-dependent mechanical behavior and stress-strain behavior dependent on the rate of strain ${ }^{23}$. The mechanisms for this behavior are from fluid flow-independence and flow-dependence. The inter-molecular friction 
from the PG matrix is responsible for the flow-independent mechanism and the interstitial fluid flow and its resultant frictional drag are responsible for the flow-dependent mechanism. The drag from fluid flow is the main viscoelastic behavior in healthy cartilage. In damaged cartilage where there is an increase in fluid and a decrease in frictional drag, there is less stress protection for the matrix components ${ }^{24}$.

Joint lubrication is also important to the mechanical properties of articular cartilage. It is thought that both fluid film and boundary lubrication are responsible for the low coefficient of

friction in synovial joints, normally around $0.001^{25}$. For fluid film lubrication of the joint surfaces to work effectively, the film must be thicker in comparison to their roughness. Film thickness is dependent on fluid viscosity, gap shape between surfaces, their velocity, and the surface stiffness. Additionally, a low coefficient of friction can be obtained without the fluid film by a mechanism known as boundary lubrication. Boundary lubrication involves adherence of molecules to the surface that are sheared instead of the fluid film. Currently, it is thought that both of these mechanisms are responsible for the low friction in articular cartilage, particularly the fluid film lubrication at high loads and the boundary lubrication at low loads ${ }^{14}$.

\subsection{CARTILAGE DEGENERATION AND REPAIR}

\section{Degeneration}

Progressive loss to the normal structure and function of articular cartilage, along with attempted repair, remodeling, and sclerosis of the subchondral bone are the general characteristics of an osteoarthritis diagnosis. There is usually an accompaniment of pain, restricted motion, grating sensation with movement, effusions, and deformity to the joint. OA progresses differently in individuals with a chain of events resulting in changes to the cells and 
matrix leading to a loss of structure and function, usually in conjunction with cartilage repair and bone remodeling ${ }^{24}$. The earliest signs of $\mathrm{OA}$ at the histological level are fibrillation of the superficial tangential zone, with some extension into the underlying middle zone; proteoglycan decreased staining in the top two zones; blood vessels extension beyond the tidemark from the subchondral bone; and subchondral bone remodeling. OA progresses from disruption of the superficial layers to surface irregularities and eventually large clefts, leading to fissures as deep as the subchondral bone ${ }^{24}$.

It is thought that many mechanisms are responsible for the loss of cartilage. Although the exact pathway is not known, researchers believe there are three stages: disturbance of the cartilage matrix, the response by chondrocytes to damaged tissue, and a decline in the response of synthesis by chondrocytes with progressive tissue loss ${ }^{24}$. In stage one, with or without fibrillated appearance, there is an alteration in the framework of the matrix or at the molecular level, leading to an increase in water. With this increase in water, there is usually a decrease in aggregating PGs, concentration of PGs, and decreased GAG length, which all leads to increased permeability and decreased stiffness.

There are a variety of causes that lead to stage one; high-intensity impact, torsional loading, accelerated matrix degenerations, or metabolic changes that disrupt the chondrocytes ability to maintain the cartilage matrix. Stage two starts when chondrocytes detect damage and osmolarity changes, charge concentration, or strain, responding with mediators to stimulate a cellular response of chondrocyte proliferation in conjunction with increased anabolic and catabolic activity. Chondrocytes may act to restore tissue, sustain the altered state, or increase the cartilage volume. Increased activity of chondrocytes can remain for years and stop the progression of OA temporarily. When there is a failure to restore the articular cartilage at this 
point, it leads to the third stage of OA development. The decline at this point results in both damage and death to chondrocytes, due to the loss of stabilization and protection from the matrix. This may be the result of damage to the tissue with disruption of the chondrocytes, creating a downregulation of the chondrocytes in response to anabolic cytokines ${ }^{24}$.

The relationship between joint usage and degeneration of articular cartilage is an integral part for comprehension of prevention and treatment of OA. Exercise such as running, even at strenuous levels, does not affect the progression of the disease when the individual has normal articulation surfaces, alignment, stability, innervation, and control of muscle. In fact, cartilage matrix synthesis is stimulated in cartilage during application of cyclical loading. However, if there is no load or application of a static load, then the cartilage begins to degrade the matrix and progress to joint degeneration ${ }^{24}$. Demanding repetitive activities will speed up the development of OA, particularly occupations with high physical demand including work in construction, mining, drill operators or any intense joint loading. Additionally, individuals with any sort of joint abnormality also have a greater risk of developing OA with normal activities of daily living 26 .

\section{$\underline{\text { Repair and Regeneration }}$}

Repair of the articular surfaces refers to restoring the articulating surface after damage, but with new tissue that does not replicate the native composition, structure, and function and restoration. Regeneration is restoration of the surface that mimics exactly normal articular cartilage ${ }^{26}$. To repair the damaged articular surface, there have been many studies and procedures developed to stop the progression and restore the articulating surface for usage. There are usually two types of defects when a repair strategy is implemented, which are partial thickness defects and full thickness defects ${ }^{24}$. Defects that are clefts without penetration down 
to the subchondral bone are partial thickness and do not heal on their own. It is thought without the subchondral penetration, there are no progenitor cells to aid with restoration. However, there is a response in the tissue surrounding the fissure, but it fails to adequately repair. Full thickness defects do penetrate down to the subchondral bone, but the fibrocartilaginous tissue repair has insufficient mechanical characteristics and over time the cartilage continues to degenerate. Even though the repair mechanism in full thickness defects is deficient, many of the current strategies are based on this process ${ }^{26}$.

Some methods used to stimulate formation of a new articulating surface include subchondral bone penetration, osteotomy, distraction of the joint, grafts of soft-tissue, transplantation of cells, addition of growth factors, and implant of artificial matrices. These procedures vary in their outcome among individuals, and the results do not replicate native articular cartilage, but there are benefits seen with a decrease in OA symptoms and better joint functioning ${ }^{24}$. Arthroscopic repairs, involving lavage and debridement, reduce pain by rinsing out the debris and eliminating the damaged tissue and along with penetration to the subchondral bone to allow for a fibrous repair. Studies done using this procedure had varying results, partially from the unpredictable nature of the fibrin clot and additional influences, particularly age and activity level ${ }^{24}$. Additionally, various factors such as lack of randomized studies, short follow-up periods, indistinct evaluations, and possible placebo effect make it difficult to describe the indications of this procedure ${ }^{7}$.

Osteotomy involves removal of a bone to re-align the joint articulation away from the damaged joint surface, particularly in the hip or knee joints. It is sometimes accompanied by debridement and penetration of the subchondral bone. Use of the osteotomy in some patients has led to decreased evidence of joint degeneration, decreased density of the subchondral bone, and 
an increase in the joint space. Osteotomy is used to decrease the loads on the most damaged articulating surface, bringing the regions that have intact articulating surface in opposition to surfaces lacking, correct joint misalignment to correct dysfunction and alleviate symptoms, and may eventual lead to formation of a new articular surface. Patients with an osteotomy tend to have pain and recurrent $\mathrm{OA}$ at long-term followup. Additional factors that can negatively affect the outcome of osteotomy results are older age, obesity, degeneration severity, joint instability, movement restriction, under or over correction with the osteotomy, and a loss post-operatively of the correction ${ }^{24,26}$.

Treatment of defects with autologous soft-tissue grafts is another procedure recognized as a potential repair mechanism for articular cartilage tissue ${ }^{7}$. The procedure involves debridement of the tissue with placement of a soft-tissue graft from a variety of sources at the defect site. Most often periosteum or perichondrium is used between the resected articular surfaces ${ }^{26}$. The rationale is that the germ layer of this tissue type has continuous chondrogenic activity and the stem cells within are capable of activation and restoration of the lesion in which it is placed ${ }^{7}$. Although results are varied, clinical trials of this procedure have some reports of a hyaline-like repair ${ }^{7}$. However, tissue grafts have yet to accomplish a complete restoration of the articular cartilage defect along with long-term stability of the repair site ${ }^{7}$.

Surgeons also have used osteochondral plugs for repair of small lesions with a procedure that has been in use for decades. In this method, a portion of healthy autologous cartilage tissue is removed from a donor site and transplanted into the portion that is damaged. This procedure does not seem to have long-term benefits, particularly since the tissue integration of the donor with the defect does not stand up to normal loading, and there is a high instance of donor site morbidity. Additionally, surgeons have used the same procedure with donor tissue from 
cadaveric specimens; however, there can be an immunological response to the tissue implanted and a possibility of cross contamination from the cadaver. Improved functioning from both procedures is limited, but young patients respond well to autologous implantation and older patients to allografts as long as the specimen is fresh with a good number of healthy chondrocytes 5 .

As an alternative to the repair of degraded cartilage, tissue engineering has been explored to replace the damaged portion of tissue. Replacement using tissue engineering approaches involves use of a combination of cells, biofactors, and scaffold material ${ }^{27}$. Cells often considered for use in engineering of cartilage tissue are: isolated chondrocytes, perichondrial or periosteal cells, synovial cells, mesenchymal stem cells (MSCs), adipose derived cells, and embryonic stem cells ${ }^{7,28}$. A supporting scaffold material is needed for seeding cells once a sufficient number of cells are cultured. Researchers consider a number of factors when choosing the type of scaffold matrix for use such as: porosity, cell adhesion, stability, biocompatibility, mechanical properties, structural organization, and availability ${ }^{7}$. Scaffolds used in tissue engineering have included: decellularized cartilage and bone matrices, collagens, collagen/hyaluronan, fibrin, hydroxyapatite, porous polylactic acid, synthetic polymers, Teflon, Dacron, agarose, and gelatin ${ }^{24,29}$. In addition to cells and scaffolds, biofactors such as transforming growth factor- $\beta$ (TGF- $\beta$ ), bone morphogenetic protein (BMP), fibroblastic growth factor-2 (FGF-2), and insulin-like growth factor (IGF-I) have been used to increase transplanted cell metabolism ${ }^{24,28}$. Through the combination of cells, scaffolds, and biofactors it is possible to create a functional tissue engineering replacement for articular cartilage. 


\subsection{TISSUE ENGINEERING}

\subsubsection{Static Cultures}

\section{Culturing of Cartilage Explants}

As mentioned previously, researchers have used explants from healthy tissue donors to restore the damaged surface for pain relief and to bring back some function ${ }^{30}$. Evaluation of culture conditions for cartilage explants is important to ensure cell viability and continued growth of cells and matrix. In a study performed by Brighton et al., healthy cartilage tissue was removed from the femoral condyle of healthy male New Zealand White rabbits ${ }^{31}$. Samples explants were dissected from the subchondral bone to a $1 \mathrm{~mm}$ thickness by 2-3 mm width by 10 mm length and evaluated for storage under various conditions. Some explants were frozen to $-180{ }^{\circ} \mathrm{C}$ by a method that freezes then thaws the samples, followed by a 7 day culture in standard medium. Other explants were cultured at 4,21 , and $37{ }^{\circ} \mathrm{C}$ in a standard tissue culture medium for 7,21 , or 60 days. The cryoprotective agents used did not penetrate enough to allow chondrocytes within the matrix to survive the freezing process. Also, the samples stored at $21{ }^{0} \mathrm{C}$ did not survive in culture, but the $37{ }^{0} \mathrm{C}$ stored explant slices remained viable for up to 60 days 31.

Culture conditions are very important to the future maintenance and growth of cartilage explants. A study that examined the addition of insulin-like growth factor I and II on the production of proteoglycans in cultured bovine articular cartilage explants found an increase in PG production in cultures with IGF-I than IGF-II over serum alone ${ }^{32}$. Strehl et al. obtained healthy explants from the femoral trochlear region of human patients undergoing patella regrooving surgery ${ }^{33}$. Samples were placed in one of the following culture mediums: serumfree medium, $10 \%$ fetal calf serum, or $10 \%$ autologous human serum medium, and cultured 
statically for $14,28,42$, or 56 days. Additionally, a perfused culture system was evaluated with the serum-free medium for the above mention time periods. Results showed samples maintained their differentiated state for up to 14 days. For long-term culture, the best results were obtained from perfused explants in the serum-free medium when compared both morphologically and histologically to fresh cartilage samples. These samples had a decrease in the amount of Collagen type II and aggrecan production, but did not increase the amount of Collagen type I produced that the static cultures exhibited when compared to normal articular cartilage ${ }^{33}$.

Ficklin et al. evaluated bovine articular cartilage explants before and after in vitro growth ${ }^{34}$. Slices were obtained from medial ridge of the patellofemoral groove and cut medial-lateral (normal and parallel to the split-line), anterior-posterior (normal), and axial (normal to the surface). Some samples were evaluated for their mechanical or biochemical properties before in vitro culture and some samples were evaluated after $14 \mathrm{~d}$ culture. For mechanical testing in confined compression, samples were loaded to 15 and $30 \%$ strain until equilibrium and a 0.1 $0.3 \%$ oscillatory strain was superimposed on each strain. The authors used the dynamic data results to obtain the $\mathrm{H}_{\mathrm{A}}$ (aggregate modulus) and $\mathrm{k}_{0}$ and $\mathrm{M}$ (permeability constants). In unconfined compression, with impermeable platens the same testing was completed as listed above, with results producing Young's modulus (E) and Poisson's ratio. Unconfined compression with a porous platen was completed with a $10 \%$ offset equilibrium strain and shear was applied with 4 cycles of 0 to $+0.5 \%$ followed by 4 cycles of 0 to $-0.5 \%$, from this data the authors determined the shear modulus. Ficklin et al. found that both aggregate modulus, Young's modulus, and the shear modulus did not depend on the direction or strain level, however Poisson's ratio was different for the various cuts, which is attributed to anisotropy. The 
authors found after in vitro growth the permeability constants stayed the same, but $\mathrm{H}_{\mathrm{A}}$ and $\mathrm{E}$ decreased $^{34}$.

\section{Culturing of Cartilage Constructs}

The various statically cultured studies completed on cartilage explants have lead researchers to experiment with culturing of cartilage tissue constructs using various cells sources, scaffolding material, and bio-additives. Early studies with static cultures of cartilage constructs have been investigated in culture conditions that usually vary in components and time period. Nehrer et al. used a Collagen type I-GAG copolymer or a cross-linked Collagen type II scaffold material seeded with isolated canine chondrocytes from the knee joint ${ }^{35}$. Scaffolds were seeded with $2 \times 10^{7}$ cells $/ \mathrm{mL}$ and cultured for 3 hours, 7 days, and 14 days. Histology revealed positive staining for safranin-O in both scaffold types indicating the presence of GAG, which was also confirmed with GAG assay results of $2 \%$ GAG (by weight). Additionally, there was a higher GAG content the longer the constructs were in culture. The Collagen type II scaffold showed cells that were more rounded than those of the type I, but this difference was thought to be most likely due to the age of the chondrocytes used in the study ${ }^{35}$.

Lee et al. evaluated MSCs photo-encapsulated in collagen mimetic peptides along with another polymer for strength and treated with a chondrogenic pathway media. Results showed increased production of GAG as well as both Collagen type II and X, compared to the cellseeded polymer alone scaffolds ${ }^{36}$. Huang et al. compared hydrogels seeded with chondrocytes against those seeded with MSCs ${ }^{37}$. Isolated cells were seeded at a density of $20 \times 10^{6}$ cells per $\mathrm{ml}$ in agarose and cultured for $14,28,42$, or 56 days. Results showed an increase in tensile properties over time for both chondrocyte and MSC seeded constructs ${ }^{37}$. Huang et al. used human MSCs seeded in agarose at the three different seeding densities of 2, 6, and $9 \times 10^{6}$ 
cells $/ \mathrm{ml}^{38}$. With the addition of TGF- $\beta 3$, all cultures illustrated markers for Collagen type II and aggrecan when analyzed with RT-PCR. The higher initial seeding constructs exhibited more cartilage markers after culturing. Histology also showed dark blue staining in the constructs using alcian blue stain ${ }^{38}$.

In a study conducted by Lee et al., canine chondrocytes were isolated from shavings from the trochlear ridges in the stifle (knee) joint ${ }^{39}$. Cells were isolated and seeded after the third cell passage in $0.5 \mathrm{ml}$ on porcine Collagen type II scaffolds (initial $9 \mathrm{~mm}$ diameter to account for shrinkage and 1.5 to $2.0 \mathrm{~mm}$ thick) at a density of $2 \times 10^{6}$ cells $/ \mathrm{ml}$. Constructs were cultured in vitro for 28 days, trimmed to a $4 \mathrm{~mm}$ diameter, and 2 constructs per dog ( 6 dogs total) were implanted in defects of the opposite knee, where no original cartilage was left intact. Sutures were used to keep the construct in the defect site. After the surgical procedure, the animals were allowed to ambulate as normal for 15 weeks prior to euthanasia. Results were evaluated with histological staining using Hematoxylin and Eosin (H \& E), Safranin-OlFast green, and monoclonal antibody for Collagen type II. Additionally, mechanical testing was performed using an indentation tester ${ }^{39}$.

Histological results showed cells distributed throughout the scaffolds, with a majority of the cells around the periphery of the scaffold, which were elongated in shape and the cells further in the scaffold were more rounded. The amount of reparative tissue in the implanted site was $88 \%$ more than untreated defects with a combination of hyaline cartilage $(42+/-10 \%)$ and fibrocartilage $(52+/-11 \%)$ filling the defect. $\quad H \&$ E staining showed hyaline cartilage near the edges of the implant, which contain positive staining for Collagen type II confirmed by immunohistochemistry, with fibrocartilage located in the central region of the construct. Safranin-O staining only demonstrated a large amount of proteoglycans in the repair tissue of 
one implant. Indentation testing revealed a 6-fold lower equilibrium stiffness and 20-fold lower dynamic stiffness than normal canine articular cartilage ${ }^{39}$.

Mauck et al. used bone marrow obtained from 3-6 month-old bovine tibia or femur to isolate mesenchymal stem cells ${ }^{40}$. Additionally, the carpometacarpal joints were used to isolate chondrocytes from the articular cartilage. MSCs and chondrocytes were expanded for 3 or 2 passages prior to seeding, respectively. Constructs were created with a 1:1 solution of agarose to cells and Dulbecco's modified eagles medium (DMEM). After gelation at room temperature for 20 minutes, constructs were punched at a diameter of $5 \mathrm{~mm}$. Every 2 week interval 3-4 samples were removed for evaluation by mechanical testing for up to $70 \mathrm{~d}$ in culture ${ }^{40}$.

Unconfined compression using 2 impermeable platens was used to perform creep testing with a $0.02 \mathrm{~N}$ force and determine the equilibrium compressive Young's modulus. Additional dynamic testing was performed after equilibrium was reached using $1 \%$ oscillatory deformation at $0.1,0.5$, and $1.0 \mathrm{~Hz}$ with dynamic modulus calculated at each frequency. The equilibrium and dynamic modulus results showed a dependency on culture time and cell type. The chondrocyte seeded constructs resulted in an equilibrium Young's modulus of 140+/-18 $\mathrm{kPa}$ and the MSC laden scaffolds $48+/-12 \mathrm{kPa}$ after $70 \mathrm{~d}$ in culture. Additionally, chondrocytes seeded scaffolds were significantly stiffer than the MSC constructs after 70d in culture. Results showed values of 1.4+/-0.2 MPa for chondrocyte constructs and 0.8+/-1 MPa for MSC constructs for dynamic modulus. ${ }^{40}$.

\subsubsection{Application of Compression}

\section{Culturing of Cartilage Explants}

Application of compressive loading to articular cartilage can further influence its structure and composition. Similar to static cultures, analysis of articular cartilage response to 
mechanical forces began with investigation into compression of articular cartilage explants. Jones et al. evaluated cartilage strips harvested from 9 month old calves placed in a petri dish with another petri dish placed on top of the samples ${ }^{41}$. Weights were placed on the top dish to statically compress the samples up to 4 days with forces of $15 \mathrm{kgfcm}^{-2}$ or $30 \mathrm{kgfcm}^{-2}$. Samples were removed at 0,2 , and 4 days and examined for their proteoglycan synthesis. Results showed that the larger, $30 \mathrm{kgfcm}^{-2}$ load significantly reduced the production, with decomposition of proteoglycans seen as early as $6 \mathrm{hrs}$ of incubation. The $15 \mathrm{kgfcm}^{-2}$ load appeared to have no significant effect on the PG content. The researchers hypothesize that the reduction from the larger load reduces water content by $20-40 \%$ thereby increasing the concentration of macromolecules in the matrix giving rise to a diminution in PG synthesis ${ }^{41}$.

Palmoski et al. used a specially designed apparatus to apply compressive stress to cartilage explants with a piston driven downward by air pressure ${ }^{42}$. Articular cartilage plugs with a $3 \mathrm{~mm}$ diameter were obtained from the femoral condyles of normal adult canines. Over a 2-hour period, explants were stimulated with static, 60 seconds on and off, or 4 seconds on and 11 seconds off with compressive loading at a load magnitude of 11,55 , or $110 \mathrm{~N} / \mathrm{mm}^{2} \times 10^{-4}$. After incubation, the cartilage was analyzed for net GAG synthesis and release of pre-labeled GAGs from the cartilage into the media ${ }^{42}$. Statically compressed cultures had a reduction in the amount of GAG synthesis by $32-50 \%$ when compared to control samples as well as the applied 1-minute cyclic stress intervals. The 4/11 duty cycle results showed dependence on the stresses applied for the GAG results with the highest synthesis from the $55 \mathrm{~N} / \mathrm{mm}^{2} \times 10^{-4}$ load. Release of newly synthesized GAGs into the media was $1 \%$ for all the stimulation sequences ${ }^{42}$.

Femoropatellar cartilage obtained from 1-2 week old bovine calves was used in a study completed by Sah et al. ${ }^{43}$. Sections were cut with a microtome removing the first $100 \mu \mathrm{m}$ 
section, cutting the plane-parallel in two $1 \mathrm{~mm}$ thick slices, punched with a $3 \mathrm{~mm}$ diameter punch, and placed in media for 2-6 days prior to application of compression. The compression chamber, constructed from polysulphone, contained wells with nubs extending up from the base to serve as the bottom compression platen attached to an actuator for application of cyclic loading. Additionally, the nubs had a depression to keep the samples in place within the chamber. Similarly extensions out of the lid protruded downward to compress the sample from above allowing free flow of media around the circumference of the explants. The lid also contained micrometers for application of a pre-displacement to explants. Cyclic compression was applied with amplitudes between 1.3 to $10 \%$ for frequencies of $0.0001,0.001,0.01,0.1$, and $1.0 \mathrm{~Hz}$ for 23 hours. Significant results were seen at higher frequencies with strains of 1 to $5 \%$ showing biosynthesis and incorporation of GAG components ${ }^{43}$.

\section{Culturing of Cartilage Tissue Constructs}

Similarly to explants, application of forces to tissue engineered constructs is thought to improve the structure and function in vitro. Waldman et al. ${ }^{44}$ isolated articular chondrocytes from 6-9 month old calves. Cells were grown in a multi-layer of approximately 10 cells thick, with a density of $1.6 \times 10^{5}$ cells $/ \mathrm{mm}^{2}$, on the surface of porous calcium polyphosphate ceramic substrates. After 1, 8, or 15 days of incubation, cultures were placed in a loading chamber in a Mach-1 ${ }^{\mathrm{TM}}$ (Biosyntech, Laval, PQ). The Mach- ${ }^{\mathrm{TM}}$ mechanical tester had a custom designed titanium alloy platen for simultaneously stimulating 4 samples. The cultures were placed in the culture dish with a $2 \%$ agarose pre-constructed cylinder resting on the top surface to prevent disbursement of cells during loading. Samples were subjected to a single application of compressive cyclic loading at one of the following amplitudes: 9.8, 19.6, or $29.4 \mathrm{mN}$ and 
durations: 15,30 , or $60 \mathrm{~min}$ with a frequency of $1 \mathrm{~Hz}$. Cultures were either immediately removed for analyses or cultured for an additional 4 weeks under static conditions ${ }^{44}$.

Results showed chondrocytes were more responsive to loading applied early on in the culture period. Samples subjected to compression after $24 \mathrm{hr}$ had an increase in collagen and proteoglycan production. Additionally, there was an improvement in the mechanical properties with a twofold increase in both the equilibrium stress and the modulus seen in the cultured samples 4 weeks after application of loading. Little difference was seen between the different amplitudes of loading and 30 minutes of exposure showed the most benefit with a high accumulation of newly synthesized matrix molecules of collagen and proteoglycans. Researchers further analyzed the media to determine if the changes were due to increased synthesis or increased retention of ECM molecules. Results showed mechanical stimulation had little effect on the release of newly synthesized macromolecules into the media ${ }^{44}$.

A study completed by Lee et al. ${ }^{45}$ used chondrocytes isolated from the knee joint of adult canines for tissue constructs. Cells were expanded and seeded into Collagen type II scaffolds obtained by a method of freeze-drying porcine Collagen type II slurry into a sheet punched for seeding. Constructs were placed in polysulfone chambers as described earlier by Sah et al. ${ }^{43}$, for application of mechanical compression after $2,7,14,30 \mathrm{~d}$ pre-culture ${ }^{45}$. Constructs were either statically compressed for $0,10,25$, or $50 \%$ strain for $24 \mathrm{hr}$ or were dynamically compressed at $0.1 \mathrm{~Hz}$ for $24 \mathrm{hr}$ at $3 \%$ strain with static offset compression of $10 \%$. Results showed an up regulation in synthesis of both protein and proteoglycan relative to statically and non-stimulated controls. However, researchers found less accumulation of newly synthesized macromolecules within the type II scaffold compared to the statically stimulated and non-stimulated controls. The authors believe the higher accumulation in the media is from a greater amount of fluid flow 
out of the scaffold, matrix disruption, and/or a greater presence of metalloproteinases leading to more matrix turnover ${ }^{45}$.

Demarteau et al. ${ }^{46}$ used human articular chondrocytes isolated from the articular surface of post-mortem knees as a cell source and a 3-D biodegradable polymer of poly(ethylene glycol teraphathalate) (PEGT) and poly(butylenes teraphathalate) (PBT) foam for the scaffold material. Chondrocytes were expanded and $5 \times 10^{6}$ cells were seeded through a perfusion bioreactor in foams with dimensions of $8 \mathrm{~mm}$ diameter and $1 \mathrm{~mm}$ thickness. Constructs were kept in culture with orbital mixing for 3 or 14 day. An additional culture of 3 days with dynamic compression was applied to some of the constructs by placing constructs in the peripheral wells of a polysulphone container. Centered above each well was a cylindrical plunger with a micrometer screw adjustment to position precisely in contact with the surface of each construct. Additionally, the container had a magnetic stir bar in the center to stir and inlet/outlet ports for media exchange. A micro-stepper motor programmed with LabVIEW software controlled displacement of plungers and recorded load ${ }^{46}$.

The deformation consisted of 6 cycles of $2 \mathrm{hr}$ sinusoidal compression with $5 \%$ strain amplitude superimposed on a $5 \%$ strain offset at $0.1 \mathrm{~Hz}$, followed by a $10 \mathrm{hr}$ rest period. Samples were evaluated histologically with Safranin-O staining and immunohistochemically with antibody recognition of Collagen types I and II. Additionally, constructs were analyzed for biochemical content of GAGs and RT-PCR assays for Collagen type II, aggrecan, and Sox9 gene 46. There was no change in amount of Collagen type II, aggrecan, and Sox 9 expression with application of compression. A change was seen between the different time periods in static cultures with an increase in Collagen type II in $17 \mathrm{~d}$ compared to the $3 \mathrm{~d}$. The synthesis, accumulation, and release of GAG corresponded to the GAG content of the constructs before 
application of compression, constructs with an accumulation of at least $5 \mu \mathrm{g}$ (GAG)/ $\mu \mathrm{g}$ (DNA) prior to compression had greater amount after stimulation compared to free-swelling controls; however, this was not seen with mRNA Collagen type II expression. Demarteau et al. concluded chondrocyte cell's response under dynamic compression was primarily modulated by the ECM surrounding the cells such as quantity and composition at the time of stimulation ${ }^{46}$.

Pelaez et al. conducted experiments using cyclic compression on human mesenchymal stem cells seeded in fibrin gel scaffolds ${ }^{47}$. Human MSCs were passaged 3 or 4 times until a sufficient number for seeding was achieved. Cells were placed in 1.0X, 1.5X, or 2.0X of fibrin gel concentrations, to a final cell count of $10 \times 10^{6}$ cells $/ \mathrm{ml}$. The combined gel with cells mixture was placed in molds of $8 \mathrm{~mm}$ diameter with a depth of $1.5 \mathrm{~mm}$. Once solidified, constructs were placed in ITS supplemented media. Constructs were allowed to incubate for 20 hours prior to placement in a bioreactor system for application of mechanical stimulation ${ }^{47}$. The bioreactor system consisted of a chamber with depressions for placement of constructs with an impermeable loading platen located above the depression. Specimens were placed between two porous filters and situated into the depression. The device had a load cell to evaluate load response and a linear variable differential transducer for determination of displacement. Designers used a cam-follower system for application of sinusoidal displacements at different frequencies and magnitudes ${ }^{48}$.

Initial testing included a static load of $5 \%$ strain applied to the $1.0 \mathrm{X}$ constructs for 5 minutes followed by a sinusoidal displacement at $10 \%$ strain for 4 hours at $1.0 \mathrm{~Hz}$ for 3 days. Concentrations of $1.0 \mathrm{X}, 1.5 \mathrm{X}$, and $2.0 \mathrm{X}$ were stimulated for 2 days with the above stimulation. Furthermore, 1.0X concentration had the same loading but with additional frequencies of 0.1 , 0.5, and $1.0 \mathrm{~Hz}$. Results showed cyclic compression maintained the cell viability within the 
constructs as compared with static controls. Analysis of gel concentrations resulted in higher fibrin gel concentrations having a negative overall effect on cell viability with $1.0 \mathrm{X}$ being the least. Additionally, cell viability was best at the $1.0 \mathrm{~Hz}$ frequency. Aggrecan synthesis increased with all cyclic compression regardless of the gel concentration or the frequency of stimulation and Collagen type II up-regulated with the $1.0 \mathrm{X}$ gel concentration and $1.0 \mathrm{~Hz}$ frequency ${ }^{47}$.

A study conducted by $\mathrm{Ng}$ et al. applied dynamic compressive loading to cartilage constructs $^{49}$. Bovine chondrocytes were isolated from 2-3 month old calves and placed in agarose gel suspensions at a density of $60 \times 10^{6} \mathrm{cells} / \mathrm{ml}$. The agarose bilayer was created by adding 3\% agarose cell gel suspension into a mold $(6.35 \mathrm{~mm}$ diameter $\times 2.3 \mathrm{~mm})$ followed by $2 \%$ agarose cell gel suspension. Cored disks (4.76 diameter x $2.3 \mathrm{~mm})$ were punched and cultured in a chondrogenic media for $2 \mathrm{~d}$ prior to mechanical stimulation. Loading of disks involved a $10 \%$ strain application at $1 \mathrm{~Hz}$ for $3 \mathrm{hr} / \mathrm{d}, 5 \mathrm{~d} / \mathrm{wk}$ for up to 4 weeks. Constructs were tested in unconfined compression with two impermeable platens with a $0.02 \mathrm{~N}$ creep tare load followed by a ramp displacement of $1 \mu \mathrm{m} / \mathrm{sec}$ to $10 \%$ strain for stress relaxation testing, results determined the bulk compressive modulus and the bulk dynamic modulus for the samples. Additionally, samples were allowed to recover after initial mechanical testing and then the compressive modulus was determined related to construct depth-dependence using optical microscopy ${ }^{49}$.

After 28 days in culture, constructs loaded dynamically had a higher bulk compressive modulus than constructs in free-swelling culture. Additionally, all day 28 cultures were stiffer than Day 0 constructs. There were also significant increases in the dynamic modulus of the cyclically loaded constructs $(0.18+/-0.03 \mathrm{MPa})$ compared to the free-swelling $(0.12+/-0.03 \mathrm{MPa})$ 
and Day $0(0.10+/-0.02 \mathrm{MPa})$. Results of the mechanical properties from the depth-dependence analysis showed a significantly softer compressive modulus in the $2 \%$ layer compared to the $3 \%$ layer. As the culture time progressed, significant increases were observed in the $2 \%$ group for both dynamically loaded and the free-swelling cultures. Application of dynamic loading revealed a significant increase in local stiffness located in the middle and bottom of the construct that was greater than its entire $3 \%$ layer and the $2 \%$ layer of free-swelling constructs ${ }^{49}$.

Lima et al. isolated chondrocytes from the carpometacarpal of bovine joints ${ }^{50}$. Chondrocytes were combined in a cell suspension with agarose for a final density of $30 \times 10^{6}$ cells in $2 \%$ agarose gel with dimensions $0.5 \mathrm{~cm}$ diameter and $0.23 \mathrm{~cm}$ thickness. Constructs were precultured for up to $56 \mathrm{~d}$ and some cultures were supplemented with TGF- $\beta 3$ for either the first 14 or 21 culture days. Constructs were loaded with unconfined compression to a $10 \%$ peak strain at $1 \mathrm{~Hz}$ for $3 \mathrm{hr} / \mathrm{d}, 5 \mathrm{~d} / \mathrm{wk}$. Upon completion of culture the constructs were evaluated for their mechanical properties with unconfined compression testing and biochemical content specifically looking at GAG and collagen content. Mechanical properties were significantly different depending on culture conditions. In the absence of TGF- $\beta 3$ the dynamic group had a $33 \%$ increase in modulus over the free-swelling group. However, with the addition of TGF- $\beta 3$ an $87 \%$ decrease was seen in modulus over free-swelling conditions. GAG values for all groups were higher, but collagen content was significantly lower for all groups compared to native $\operatorname{articular~cartilage~}^{50}$.

Application of dynamic compression to tissue-engineered constructs during in vitro culture is important to produce many of the necessary properties for articular cartilage. The above studies show that cyclic compression can produce increases in GAG and collagen content from the construct cells necessary to lay down the proper matrix framework that is needed for 
appropriate cartilage functioning. Additionally, many stimulation profiles can also increase the mechanical properties making the construct stronger and able to withstand forces more closely associated with normal articular cartilage functioning.

\subsubsection{Application of Tension}

\section{Culturing of Chondrocytes}

Studies with application of tensile forces are less prominent due to more difficulty applying tension in culture conditions. Toyoda et al. ${ }^{51}$ examined application of tensile load to isolated chondrocytes obtained from Japanese White rabbit knees ${ }^{51}$. Cells were seeded on to a Flex-I plate from Flexcell ${ }^{\circledR}$ Corp. (McKeesport, PA) at a concentration of 5 x $10^{5}$ cells per plate. A custom system was built to apply force to the plates consisting of an acrylic chamber connected to vacuum pump. The system also included a solenoid valve, pressure regulator, pressure gauge, and an electric timer that control frequency, duration, and magnitude. When the chamber was evacuated the base of the plate deformed as the surface was stretched. Previous research showed equibiaxial strain existed at the center of the plate where radial and circumferential strain were equal ${ }^{51}$.

Cyclic strain was applied with a vacuum force of $80 \mathrm{~mm} \mathrm{Hg}$ for $24 \mathrm{hrs}$ with $3 \mathrm{sec}$ of applied load and $3 \mathrm{sec}$ rest. Analysis of the data showed after stretching a 7.7\% increase in the total cell area. Additionally, the cells at the highest point of load (periphery) exhibited elongation and alignment perpendicular to the application of tension. Collagen synthesis increased significantly in stimulated plates and an increase of tissue inhibitors of metalloproteinases, which can break down the matrix. Furthermore, no change in PG content was seen between stimulated and non-stimulated plates ${ }^{51}$. 
Honda et al. isolated chondrocytes from Japanese White rabbits and seeded at a density of $5 \times 10^{4}$ per $25 \mathrm{~mm}$ silicon Flexcell ${ }^{\circledR}$ plate type 1 (stretch) and 2 (non-stretch) (Flexcell ${ }^{\circledR}$ Corp., McKeesport, PA) including Collagen type II to ensure cell attachment to plates ${ }^{52}$. A Flexcell ${ }^{\circledR}$ strain system was used to apply the loading to the plate. Similar to the custom-built system described above by Toyoda et al. ${ }^{51}$, the Flexcell@ culture system consists of a plate or plates composed of a flexible membrane situated on top of a vacuum pump. When the vacuum is applied to the plates the membrane is stretched and strain is applied to the cells on the membrane. A computer system is used to control the frequency, magnitude, and duration of an applied load.

Honda et al. used a $17 \mathrm{kPa}$ load applied to the plate cyclically at a frequency of 30 cycles/min ${ }^{52}$. Results showed cells of a spindle-like shape with an alignment perpendicular to the application of loading. Histological staining with toluidine blue exhibited intense staining for Collagen type II on type 1 and 2 plates except for the region in the center of the plate on type 1 where the load was greatest. Immunostaining showed similar results with loss of staining in the center of type 1 plates indicating loss of proteoglycan and Collagen type II. The total PG incorporation was lower for type 1 plates than type 2 plates at $6 \mathrm{hr}$, but became negligible at 12 hr. The mRNA of Metalloproteinases (MMP) 1, 3, 9 and tissue inhibitor of metalloproteinases (TIMP) 1 increased on the type 1 plates, but was not seen on type 2, but there was no difference between the plate types for mRNA of MMP-2 and TIMP-2. The results of the MMP and TIMP assay indicate that there is destruction of cartilage components with high magnitude cyclic loading as seen with OA patients ${ }^{52}$.

Fukuda et al. investigated chondrocytes isolated from 10 month old calves seeded on Flexcell ${ }^{\circledR}$ plates coated with Collagen type $\mathrm{I}^{53}$. Cells were seeded at a density of $2 \times 10^{4}$ 
cells $/ \mathrm{ml}$ and cultured statically for 4 days before application of mechanical stimulation. Plates were used with the Flexcell ${ }^{\circledR}$ Strain instrument described above. Chondrocytes were stretched repeatedly for 3 seconds and then relaxed. For lower strain the plates were stretched every 6 minutes stretched to $5 \%$ and for higher strain at 10 cycles per minute, stretched for 3 seconds to 17\% elongation with 3 seconds of relaxation in between cycles. Fukuda et al. found that at low frequency and stretch the PG content was enhanced, however, high frequency and stretch reduced the PG content ${ }^{53}$. High stress conditions were found to enhance the DNA content indicating PG content was not effected by cell toxicity. Protein kinase, thought to be involved in somehow in the process of OA, significantly decreased for all tension stimulation conditions ${ }^{53}$.

DeWitt et al. applied tension to primary cultures of chondrocytes fashioned into a tissue by allowing the cells to form a 5-8 cells thick layer ${ }^{54}$. Chondrocytes isolated from 13-day-old embryonic chicks were placed at a density of $15 \times 10^{6}$ cells $/ 25 \mathrm{~mm}^{2}$ in plastic culture flasks. After 14 days, when the cultures had achieved the required cell thickness, they were removed intact from flask and divided in half with one portion placed in static cultured as a control and one for placement in the cyclical stretching apparatus. Briefly, cell sheets were clamped on either end and placed in tissue culture flasks with the top portion cut out. One clamp remained stationary and the other was displaced by shaft connected to a rotating axle and by rotation of the central axle, each clamp was displaced. Positioning of the clamp determined the amount of displacement applied to the cell sheets, with a maximum amount of $1 \mathrm{~cm}$ displacement. A variable speed gear box controlled the frequency of stretching ${ }^{54}$.

Mechanically stimulated cultures were allowed to equilibrate for 24 hours once placed in the apparatus. Cultures were adjusted to zero tension and a $5.5 \%$ strain was imposed at a frequency of $0.2 \mathrm{~Hz}$ for 24 hours. Results showed an increase in incorporation of GAG 
precursors in cultures subjected to mechanical stimulation. Additionally, cultures cyclically loaded for $24 \mathrm{hrs}$ showed an increase in DNA synthesis as compared to static cultures. The size of the GAGs synthesis was analyzed and researchers found a slight increase in molecule size in cultures subjected to mechanical stimulation. Evaluation of cyclic AMP levels showed an increase in cyclically stimulated cultures, probably due to distortion of the cells, which activates prostanoid synthesis, up regulating cAMP, indicating an up regulation in synthesis of proteoglycans, protein, and RNA ${ }^{54}$.

\section{Culturing of Cartilage Tissue Constructs}

Evaluation of the effects of tension on cells seeded in a scaffold better mimic native tissue. Yang et al. separated chondrocytes from 17-day-old embryonic chick sterna and expanded up to $5 \times 10^{6}$ cells $/ \mathrm{ml}^{55}$. Gelfoam sponges (Dupont, DE) cut into squares of $2 \times 2 \mathrm{~cm}$ and seeded with $100 \mu \mathrm{l}$ of cell suspension. Constructs were placed in a Bio-Stretch (ICCT Technologies, Ontario, Canada), which contains a clamp on one side to hold the construct edge and a mobile clip containing a metal bar, on the opposite end for grabbing the construct and applying tension ${ }^{55}$. A computer program called Bio-Stretch Manager adjusts the Bio-Stretch controller that manipulates magnets to apply stretch ${ }^{56}$. Constructs were stimulated at $1.0 \mathrm{~Hz}$ for $48 \mathrm{hr}$ at 5\% strain with high strain applied at the edges and low strain applied at the center.

Upon completion of stimulation, constructs had $2 \mathrm{~mm}$ removed from the center and peripheral regions for analyses. RT-PCR examination of Collagen type X levels showed an increase in both the clip and clamp with the center at low levels similar to non-stimulated. Western blot also confirmed an up regulation in Collagen type X protein levels. Markers made on the construct showed significant differences in strain profiles of the edges and center, with the edges receiving a 3 fold increase in strain. Researchers conducted additional testing with 
different applied strains of $2.5,5$, and $7.5 \%$ and found that only the center region in the $7.5 \%$ strain profile had significant production of Collagen type X. The $7.5 \%$ strain translates to $3.75 \%$ strain in the center indicating that this is the least amount of strain necessary for increased synthesis of Collagen type $\mathrm{X}^{55}$.

Vanderploeg et al. cultured immature bovine chondrocytes isolated from the femoropatellar groove ${ }^{57}$. Custom shaped polycarbonate molds were used to seed cells at $5 \times 10^{6}$ cells/ml in fibrin gel and cultured in static conditions prior to application of oscillatory tensile loading. Holes were punched in each end for application of load with a custom-made device. Briefly, the device contained a pole to secure the construct through one of the punched holes and a pole attached to a rake was placed in the second hole, which contained 8 tissue stimulation stations, to apply tension to all constructs simultaneously. Linear slides attached the rake to a DC motor to control the frequency and amplitude of tension application through an adjustment of the slides and the input voltage ${ }^{57}$.

Preliminary studies completed on constructs pre-cultured for 1,7 , or 14 days and stimulated for 68 hours at $1.0 \mathrm{~Hz}$ for $10 \%$ strain resulted in a relative insensitivity to strain magnitude with no significance in biosynthetic response, even when increased up to $20 \%$. A followup study pre-cultured constructs for 7 days and stimulated for only 48 hours at $1.0 \mathrm{~Hz}$ for $10 \%$ strain. Analysis of cell viability was high for all culture duration after mechanical stimulation. However, cells in the 7-day pre-culture developed a more stellate morphology. Oscillatory tensioned constructs showed twice as many cells exhibiting cytoskeletal projections than static cultures. Increased GAG content was seen in constructs subjected to 48 hours of mechanical stimulation, which was not seen in the 68-hour group. However, DNA content was increased in the 68-hour group, but was not seen in the 48-hour stimulated constructs ${ }^{57}$. 
Mesenchymal stem cells isolated from Wistar rats were seeded in a Collagen type IGAG scaffold researched by McMahon et al. ${ }^{58}$. Constructs were seeded at $2 \times 10^{6}$ cells $/ \mathrm{ml}$ on the dry scaffold, which was attached to silicone and clamped in the load frame of a uniaxial stretching device. After 5 days of static culture, constructs were subject to $10 \%$ strain at $1 \mathrm{~Hz}$ for 7 days. Results indicated an increase in GAG synthesis in stimulated compared to nonstimulated constructs. Furthermore, researchers evaluated the mechanism behind the increase in GAG synthesis in stimulated constructs by evaluating with a SA (stretch activated)-ion channel blocker. There was a decrease in GAG synthesis with the SA-ion blocker, but overall there was still an increase in GAG synthesis indicating that mechanotransduction pathways other than the SA-ion channel are also involved in synthesis ${ }^{58}$.

\section{Culturing of Tendon Tissue Constructs}

Application of tensile mechanical forces is also seen in tissue engineering of other soft tissues. Nirmalanandhan et al. evaluated MSCs obtained from the iliac crest of New Zealand White Rabbits to create tendon constructs ${ }^{59}$. Isolated cells were seeded on to Collagen type I sponge or Collagen type I gel at a density of $0.14 \times 10^{6}$ cells/construct in a custom-designed silicon plate with posts at either end of an elongated well for affixing the construct. Constructs were pre-cultured for 2 days prior to mechanical stimulation to allow for cell attachment to scaffolds and place in a pneumatic mechanical stimulation system. The system entailed 5 stations mounted inside an incubator that was computer controlled. Application of tensile loading was applied by stretching the plates with a pneumatic cylinder defined by amplitude, frequency, and rest between cycles. Additionally, an LVDT was attached to record the displacement data by monitoring the edge-toedge plate displacement ${ }^{59}$. 
Researchers evaluated constructs for overall change in dimensions and found that mechanical stimulation did not increase the size of the samples. The biomechanical properties of the cells seeded in collagen sponges, tested by application of constant tensile strain at $10 \% / \mathrm{s}$, had a significant improvement in the mean linear modulus $(0.016+/-.004 \mathrm{MPa})$ and linear stiffness $(0.048+/-.009 \mathrm{MPa})$ over the non-stimulated constructs $(0.005+/-.001 \mathrm{MPa}$ for modulus and 0.015+/-.004MPa for stiffness). Stimulated cell seeded collagen gel samples $(0.03+/-.014 \mathrm{MPa}$ for modulus and 0.021+/-.009 MPa for stiffness) did not have any improvement over their statically (0.021+/-.005 $\mathrm{MPa}$ for modulus and $0.011+/-.003 \mathrm{MPa}$ for stiffness) cultured counterparts. Failure mode results varied with two thirds of samples failing near the grips and one third near the central region. Overall, only favorable results were found in the cell seeded Collagen type I sponges ${ }^{59}$.

In a separate study conducted by Chokalingam et al., bone marrow was harvested from the mice and isolated for MSCs ${ }^{60}$. Cells were expanded and seeded at a density of $0.5 \times 10^{6}$ cells/scaffold on Collagen type I sponge scaffolds. After 2 days in static culture, each construct was assigned into one of the following stimulation categories: Day 0 non-stimulated, day 7 nonstimulated, day 7 stimulated, day 14 non-stimulated, and day 14 stimulated ${ }^{60}$. Constructs were stimulated using the same silicon plates and apparatus as described above in Nirmalanandhan et al. 59. Cells in stimulated constructs regardless of time period were elongated when examined with fluorescent stain, however they were randomly oriented with no regard to stimulation direction. Collagen type I gene expression was seen as a 12-fold increase in stimulated constructs compared to non-stimulated constructs. Linear stiffness was increased in stimulated constructs at a 2.3 fold increase for day 7 and a 1.6 fold increase for day $14^{60}$. 
In a study conducted by Abousleiman et al., investigators used MSCs obtained from Wistar Rat bone marrow and isolated the cells ${ }^{61}$. Cells between passages 3 and 5 were used for seeding in a decellularized human umbilical cord at dimensions of $6.5 \mathrm{~mm}$ diameter by $0.75 \mathrm{~mm}$ thickness at a density of 1 million cells/ml. After static culture for 1 hour, constructs were placed in bioreactor for application of $2 \%$ strain for $1 \mathrm{hr} /$ day at $0.0167 \mathrm{~Hz}$ and cultured for 1 to 2 weeks. Constructs were evaluated for fiber alignment, cell shape, and tensile mechanical properties upon completion of stimulation. The stimulated constructs had fibers in a parallel orientation and the non-stimulated had fibers in a more random pattern at both time periods. Additionally, the cells in the stimulated constructs had a shape more spindle-like and the nonstimulated more rounded. Stimulated constructs at 2 weeks had the highest value for modulus of elasticity $(9.91+/-2.22 \mathrm{MPa})$ compared to the non-stimulated constructs $(7.32+/-2.37 \mathrm{MPa})^{61}$.

Application of tension stimulation to tissue-engineered constructs during in vitro culture can aid in achieving a more cartilage like product. The above studies show that cyclic tension can direct the laying down of collagen fibrils in an aligned pattern, which is similar to that seen in the STZ layer of articular cartilage. Additionally, constructs treated with cyclic tensile loading have exhibited cells in a more spindle-like shape, which is also the type of cells in the STZ layer of articular cartilage. Tensile properties are also improved with application of tensile loading. Through tensile stimulation profiles constructs can achieve properties that are similar to normal articular cartilage.

\subsection{SPECIFIC AIMS}

With the incidence of $\mathrm{OA}$ on the rise, it is necessary to find a proper repair for cartilage degradation. The complex structure and properties of cartilage make it difficult to self heal or 
repair with current surgical procedures. To engineer a cartilage specific tissue it is necessary to consider the cell source, the type of matrix material, and the culturing environment. Replacement tissue may be possible through application of forces to tissue-engineered constructs in vitro. The STZ is an integral part of maintaining intact cartilage tissue. Evaluation of bovine osteochondral plugs in confined compression with the STZ portion removed via microtome showed an increased creep rate indicating increased fluid flow from increased permeability ${ }^{62}$. Once this layer is disintegrated, it affects fluid flow through increased permeability, which leads to decreased resistance to compressive forces. The deterioration process eventually destroys cartilage tissue down to the calcified zone. Early lesion detection and replacement of articular cartilage's STZ layer with a tissue-engineered replacement product could prevent the degradation process. This study focuses on engineering a tissue construct similar in structure and function to that of the STZ layer through use of a cells, scaffold material, and culture conditions that include biaxial application of load. The overall hypothesis is that with the application of both tension and compression to cell-seeded constructs, construct characteristics will be improved over static culture to become more similar to the STZ. The specific aims to test this hypothesis are as follows:

1-To create a tissue engineered construct using mesenchymal stem cells isolated from bone marrow aspirates of total hip replacement patients. Mesenchymal stem cells (MSC) were selected as the cell source due to their ability to manipulate their pathway into various connective tissue lineages ${ }^{63}$ and for the ease of obtaining them from total hip replacement patients for this study and as an autologous source for these constructs to be implanted clinically. MSCs have produced both cartilage components of proteoglycan and Procollagen type II in culture when treated with chondrogenic media ${ }^{64}$. Seeding of MSCs in a 3-D environment has previously 
shown cells changed to a round chondrocyte-like morphology, in addition to producing appropriate extracellular products ${ }^{37,65,66}$.

2-To apply stimulation protocols involving application of compression to stimulate the cells to induce synthesis of PGs and collagen type II. Previous studies on cartilage constructs showed dynamic loading produced an increase in proteoglycan and extracellular matrix synthesis 49,67,68, particularly over static culture. Additionally, compressive force improved material properties when cyclically loaded to cell seeded constructs seen with increased modulus ${ }^{49,69}$.

3-To apply tensile stimulation to induce cellular alignment similar to the superficial tangential zone of articular cartilage. Previous studies on fibroblast showed cyclic stretching elongated cells along the direction of stretch.

Application of both compression and tension stimulation to cartilage tissue engineered constructs is novel and has not been explored using this method. 


\section{CHAPTER 2 MATERIALS AND METHODS}

\subsection{BRIEF OVERVIEW}

To create a cartilage construct to mimic the superficial tangential zone of articular cartilage, three different stimulation algorithms of biaxial (compression and tension), and biaxial (compression and tension) with a static offset, and tension only were applied to cell seeded constructs for comparison to one another and to non-stimulated constructs. Constructs from all stimulation algorithms were evaluated for their structure and composition by mechanical testing with indentation and tension testing, light and scanning electron microscopy, and western blot analysis. Each stimulation algorithm had a total of 12 samples as well as Day 0 constructs; 9 evaluated mechanically with indentation and tensile testing, 2 evaluated with scanning electron microscopy (SEM), 2 evaluated with histology and used for alignment, and 3 evaluated with western blot analysis.

\subsection{CELL ISOLATION AND PRECULTURE}

Bone marrow was obtained from primary total hip replacement patients (age range 35-85 years old), harvested with Virginia Commonwealth University's Institutional Review Board approval, and placed in heparin to prevent clotting, with each harvest assigned a number for tracking during the study. The suspension was transferred to $15 \mathrm{ml}$ conical tube (US Scientific, Inc. Ocala, FL), spun down at 2500 revolutions per minute (RPM) in an IEC Centra CL2 centrifuge (Thermo Fisher Scientific Inc, Waltham, MA), and the supernatant placed in T-75 flask (US Scientific, Inc. Ocala, FL). A media containing high glucose Dulbecco's Modified Eagle Medium (DMEM) (Invitrogen Corp., Carlsbad, CA) supplemented with $10 \%$ heatinactivated Benchmark $^{\mathrm{TM}}$ Fetal Bovine Serum (Gemini Bio-Products, West Sacramento, CA) and 
antibiotic/antimycotic (Invitrogen Corp., Carlsbad, CA) was added to each flask. After 2-3 days, flasks were rinsed with 3 washes of phosphate buffered saline (PBS) (Invitrogen Corp., Carlsbad, CA) to remove all non-adherent cells ${ }^{65}$. Fibroblast-like cells formed on the culture flask surface and were expanded, using trypsin (Invitrogen Corp., Carlsbad, CA) to remove the adherent cells, at a 1:3 expansion for 2 passages and 1:2 for 1 passage for a total of 3 passages prior to scaffold seeding.

\subsection{CONSTRUCT SEEDING}

Expanded cells were seeded onto sterile fibrous bovine collagen type I pads (Figure 2.1)

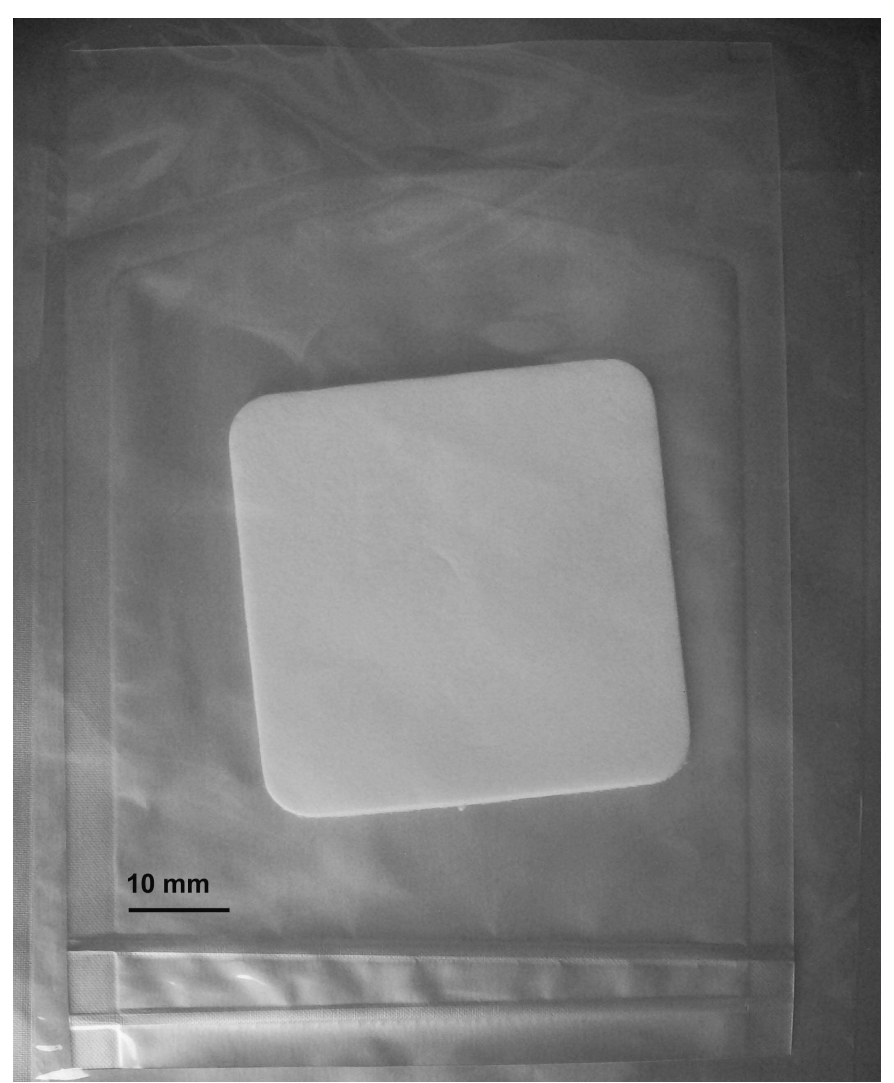

Figure 2.1 Photograph of Kensey Nash Collagen Type I scaffolding in sterile packaging.

from (Kensey Nash, Exton, PA) cut to rectangular dimensions length range $10 \mathrm{x}$ width range 20 $\mathrm{x}$ thickness range $2.0 \mathrm{~mm}$ in $50 \mathrm{ml}$ conical vial (VWR, West Chester, PA). Cells were seeded at 
an approximate density of $2.0 \times 10^{3}$ cells $/ \mathrm{mm}^{3}$ in $2 \mathrm{ml}$ of cell/chondrogenic media mixture. The chondrogenic media contained DMEM with antibiotic/antimycotic added, 1:100 dilution of ITSpremix (BD Biosciences, San Jose, CA), which is an aqueous solution containing human recombinant insulin $(12.5 \mathrm{mg})$, human transferrin $(12.5 \mathrm{mg})$, selenous acid (12.5 $\mu \mathrm{g})$, BSA (2.5 $\mathrm{g}$ ), and linoleic acid (10.7 $\mathrm{mg}), 1 \mathrm{mM}$ sodium pyruvate, $50 \mu \mathrm{g} / \mathrm{ml}$ ascorbate, $40 \mu \mathrm{g} / \mathrm{ml}$ proline, $100 \mathrm{mM}$ dexamethasone, and $10 \mathrm{ng} / \mathrm{ml}$ TGF- $\beta 1{ }^{65}$ previously shown to induce chondrogenic differentiation with the MSCs . Collagen scaffolds were placed in a 50ml conical vial (VWR, West Chester PA) and the cell/chondrogenic media mixture was added via pipette on the cut dry scaffolds and then placed on a shaker for $1 \mathrm{hr}$ to ensure cell penetration into the central region of the scaffold. Each construct was assigned a number and letter designation, with the number representing the cell source and the letter distinguishing between each construct from the same cell line. Constructs were then placed in 6 well plates (VWR, West Chester PA) with media

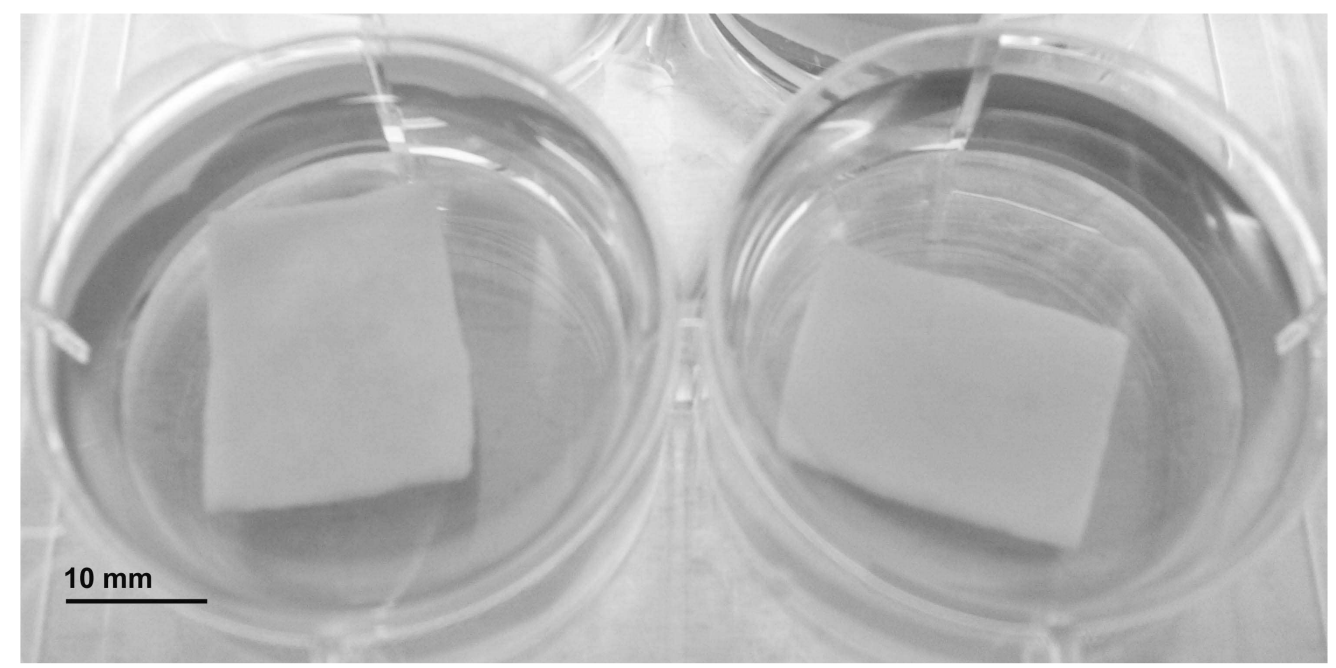

Figure 2.2 Photograph of seeded constructs in chondrogenic media within a 6-well plate.

(Figure 2.2). Media changes were performed every 3-4 days. After 1 week in static culture to allow cells to firmly attach and grow, seeded constructs were either evaluated for Day 0 results 
or 3 constructs at a time were loaded in the tissue stimulation bioreactor for application one of the stimulation algorithms.

\subsection{OPTIMAL SEEDING DENSITY AND STATIC CULTURE TIME}

A preliminary study was completed on the optimal seeding density for bone marrow cells from primary total hip replacement patients (age range from 38-68 years old). Cells were seeded on collagen type I scaffolds (Kensey Nash, Exton, PA), cut to square dimensions approximately $20 \times 22 \times 1.5 \mathrm{~mm}^{3}$ to achieve either a final seeding density of $2 \times 10^{3}$ cells $/ \mathrm{mm}^{3}$ or $4 \times 10^{3}$

cells $/ \mathrm{mm}^{3}$. Constructs were placed in chondrogenic media ${ }^{65}$ and cultured for 1,2 or 3 weeks. Each density had 2 constructs for each week. After culture, they were visually inspected for appearance change and a measurement of thickness (h) obtained. Constructs were cut with a circular punch for a final diameter of $7 \mathrm{~mm}$ and placed between 2 circular steel meshes within an acrylic holding chamber. The chamber was attached to an apparatus via polyethylene tubing and the permeability was evaluated by using flow of phosphate buffered saline through the device. Each specimen was tested 3 times at different pressure drops $(\Delta \mathrm{P})$ while flow $(\mathrm{Q})$ was measured (flow was calculated by the amount of fluid that passed through the construct in 60 seconds time), and permeability (k) calculated using Darcy's Law:

$$
k=\frac{Q h}{A \Delta P}
$$

By using this theory with known values for: h-the height the of the porous body (the construct), A-the cross-sectional area of the porous body, Q-resulting flow rate, and $\Delta \mathrm{P}$-the pressure 
difference driving the flow, then the permeability $(\mathrm{k})$ of the tissue can be calculated ${ }^{70}$. In our experiment, the pressure was applied by the amount of fluid place in the funnel to flow through the tissue (Figure 2.3) and by using a known volume, the amount of fluid to flow thorough the tissue in 60 seconds was measured and $\mathrm{k}$ was calculated from the resultant values.

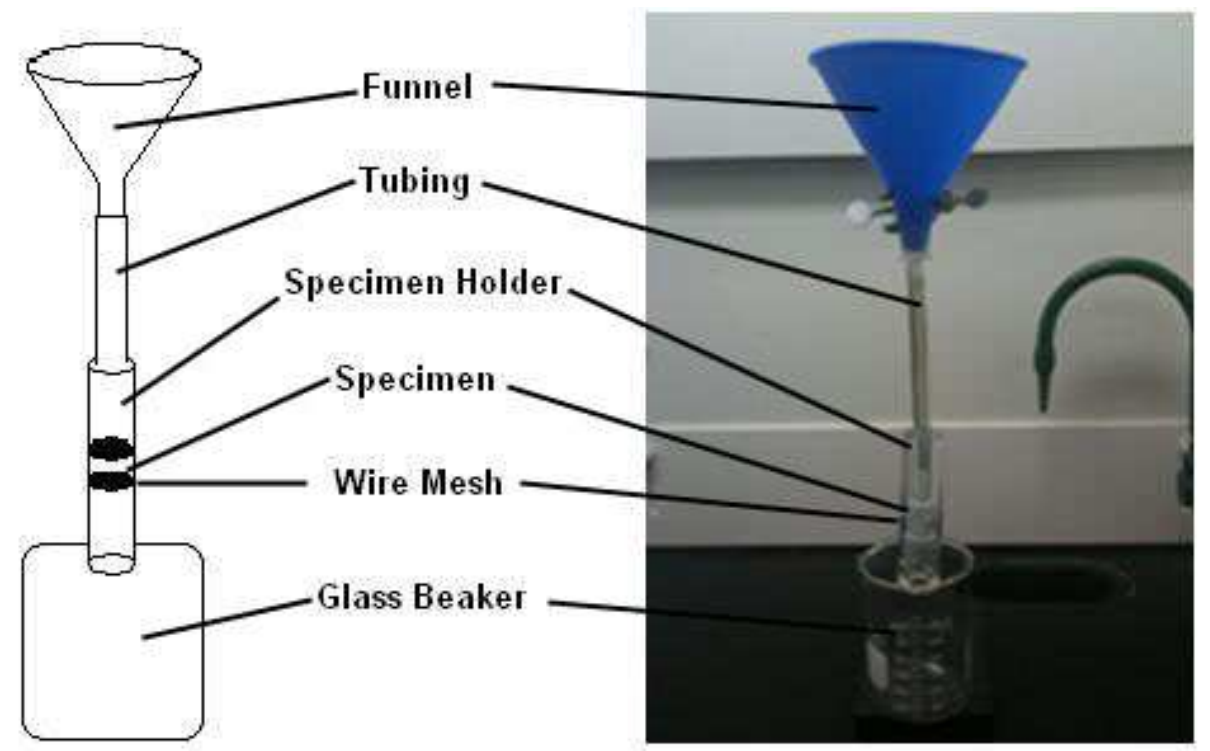

Figure 2.3 Schematic and image of the permeability testing apparatus.

Permeability values were averaged for each seeding density and culture time, and statistical comparisons made via an ANOVA to evaluate the effects of density and culture time on construct permeability ${ }^{71}$. Constructs from weeks 1 and 2 were fixed in $10 \%$ formalin. Alcian Blue Staining Kit (Poly Scientific, Bay Shore, NY), which stains strongly acidic mucopolysaccharides blue, nuclei pink to red, and cytoplasm pink, was used to evaluate constructs.

Constructs at both densities were visually assessed and appeared to contract over the culture period. With the numbers performed, a trend could be observed with high pressure exhibiting low permeability and low pressure exhibiting high permeability (Figure 2.4). Increasing culture time decreased permeability for samples seeded at low density as matrix 
production increased. At the higher density, a decreased permeability was seen with respect to culture time, except for 3-week cultures. The increase in permeability for 3-week cultures may be due to the collagen scaffold size limiting the area of cell expansion and increased cell death, decreasing matrix production. Further investigation is necessary. There was also no difference seen in the permeability between the two densities. Therefore, a lower density seeding is sufficient with longer culture time to increase matrix content. Gross visual assessment of the histological comparison showed more stained cells, matrix, and mucopolysaccarides for longer culture time (Figure 2.5).

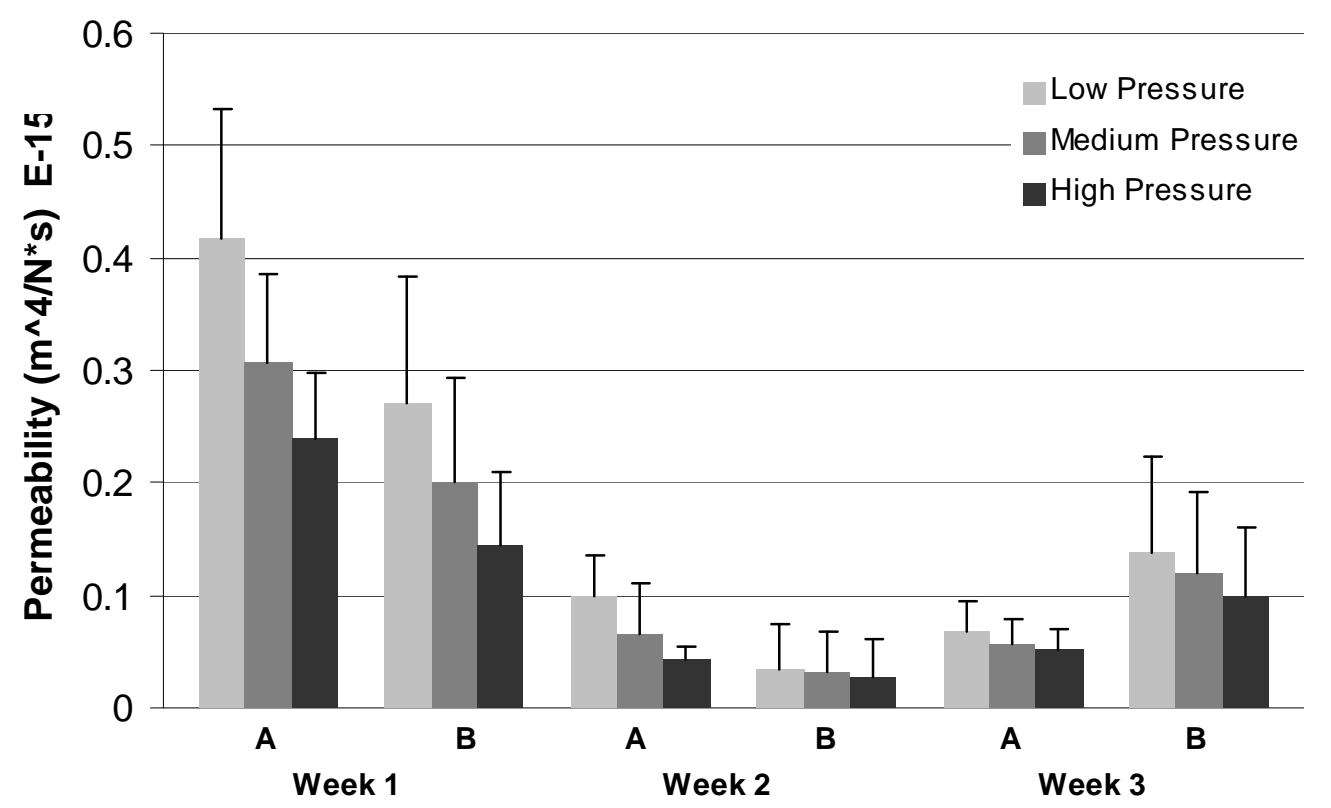

Figure 2.4 Permeability values for the 3 culture times, 2 seeding densities $\left(A=2 \times 10^{3}\right.$ and $B=4 \times 10^{3}$ cells $\left./ \mathrm{mm}^{3}\right)$, and 3 pressures. 


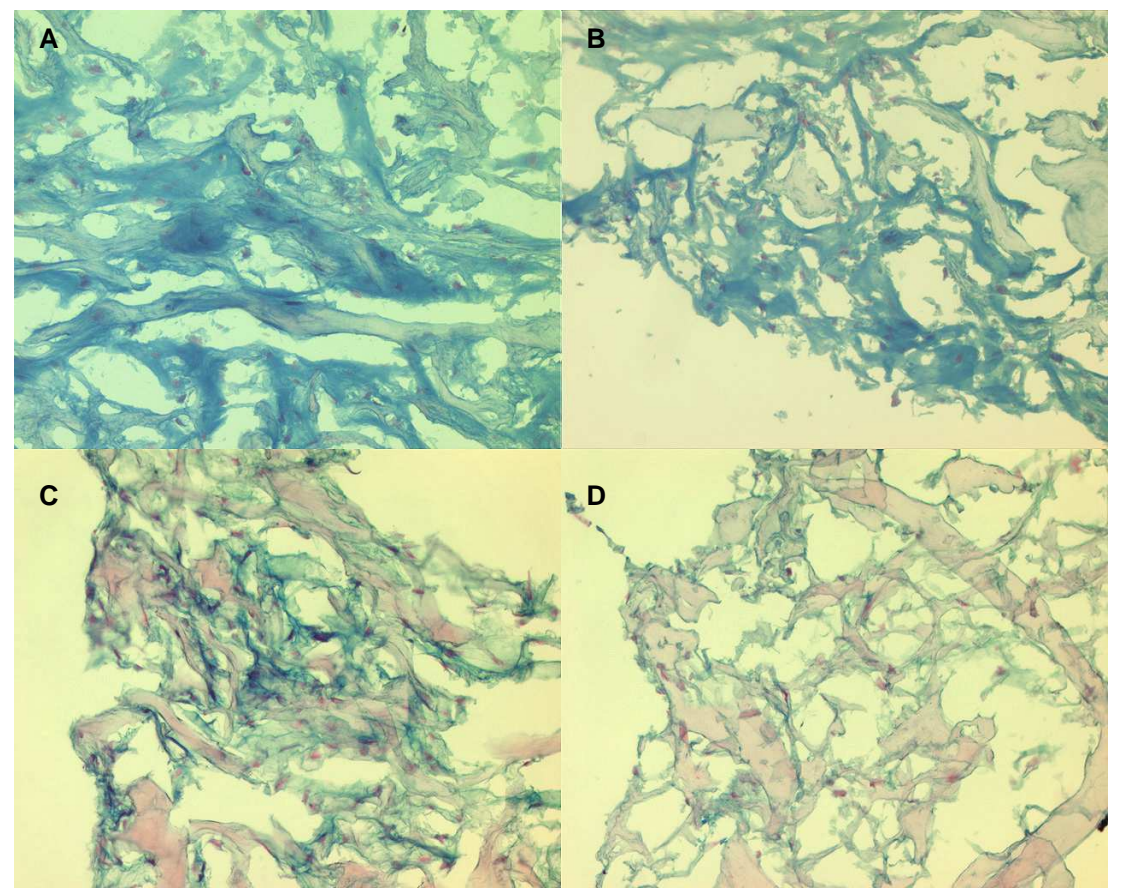

Figure 2.5 Alcian blue histological pictures of constructs. A) Higher density at week 2, B) lower density at week 2, C) higher density at week 1 , and D) lower density at week 1. (10X)

All tests showed cartilage constructs permeability was within the range for native articular $\left(1.1 \times 10^{-15}\right.$ to $\left.7.6 \times 10^{-15} \mathrm{~m}^{4} / \mathrm{N}-\mathrm{s}\right)$ cartilage ${ }^{72}$. Permeability on cultures greater than 2 weeks was lower due to the presence of more matrix components. Lower-density constructs were selected for the main study due to the results achieved above, but used at week 1 to avoid excessive shrinkage before placement in the tissue stimulation chamber.

\subsection{BIOREACTOR AND MECHANICAL STIMULATION}

The bioreactor ${ }^{73}$ was primarily composed of clear plastic material acrylic or polycarbonate to prevent corrosion of assembly components and to withstand ethylene oxide sterilization. The bioreactor contained an acrylic base stage for attachment of a tissue stimulation chamber, a compression assembly, and a tension assembly. Briefly, pistons were connected to actuators to provide linear movement along each axis with control provided by 
LABVIEW software application (National Instruments Corp., Austin, TX). The tension assembly had grips to clamp the specimen in place on a platform to support the tissue during application of compression. The tissue stimulation chamber was modified to stimulate 3 specimens simultaneously. The chamber was further improved to provide fluid flow through the chamber with a Fisher Scientific Variable-Flow Peristaltic Pump (Fisher Scientific, Pittsburgh, PA). The pump provided fluid flow through the chamber at a rate of $8.2 \mathrm{ml} / \mathrm{min}$ for 8 hours followed by no flow for 4 hours repeated twice daily. Chondrogenic media changes were performed on the chamber every 3-4 days (Figure 2.5).

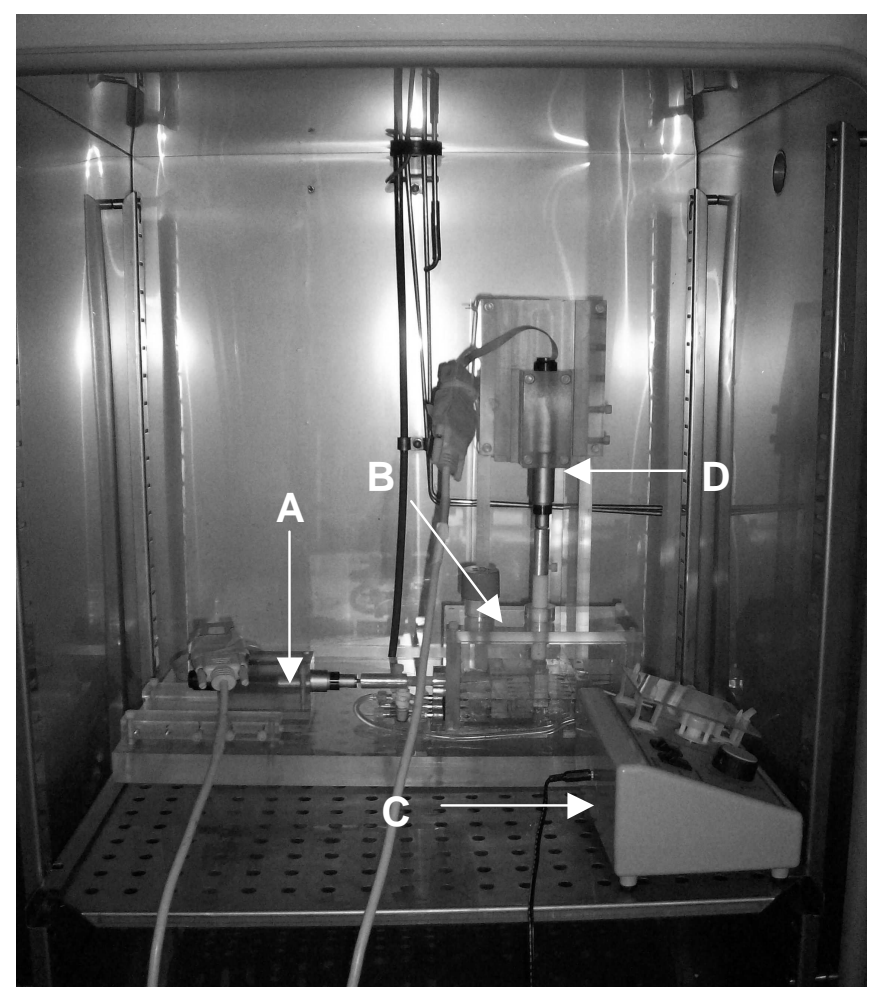

Figure 2.6 Photograph of the bioreactor within the incubator with A) tension actuator, B) tissue stimulation chamber, C) compression actuator, and D) peristaltic pump.

The bioreactor was placed in an IR Autoflow Stacked $\mathrm{CO}_{2}$ incubator (NuAire, Inc., Plymouth, $\mathrm{MN}$ ) at $37^{0} \mathrm{C}$ with $5 \% \mathrm{CO}^{2}$. One of the three stimulation algorithms was applied: 1) $10 \%$ cyclic compression applied for 1 hour followed by a 2 hour rest then application of $10 \%$ 
cyclic tension for 1 hour followed by a 3 hour rest and repeated twice (Figure 2.6); 2) 5\% offset compression with application of 5\% cyclic compression for 1 hour followed by a 2 hour rest then application of 5\% offset tension with application of 5\% cyclic tension for 1 hour followed by a 2 hour rest (Figure 2.7); or 3) $10 \%$ cyclic tension applied at a rate of $0.5 \mathrm{~mm} / \mathrm{sec}$ for 1 hour of stimulation followed by a 3 hour rest and repeated twice more (Figure 2.8). The percent of stimulation applied for compression was determined from the initial construct height and the tension offset was determined from the distance between of the exposed construct not in the tension clamps.

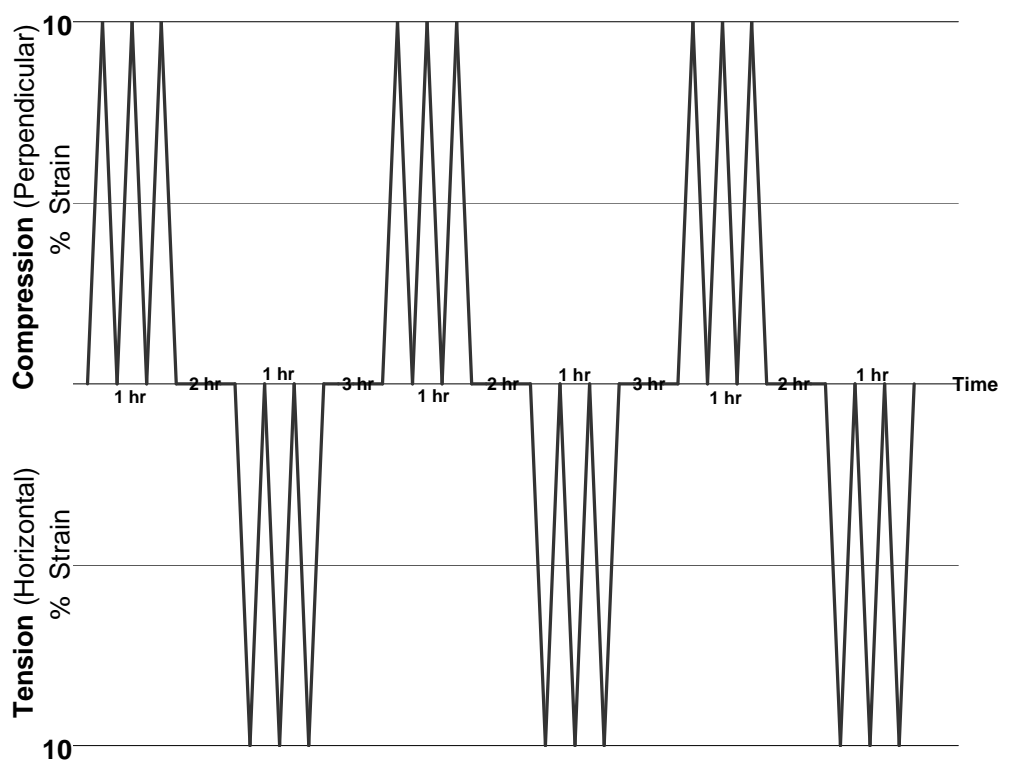

Figure 2.7 Schematic of the stimulation algorithm for stimulation 1 at $10 \%$ cyclic strain stimulation for both compression and tension. 


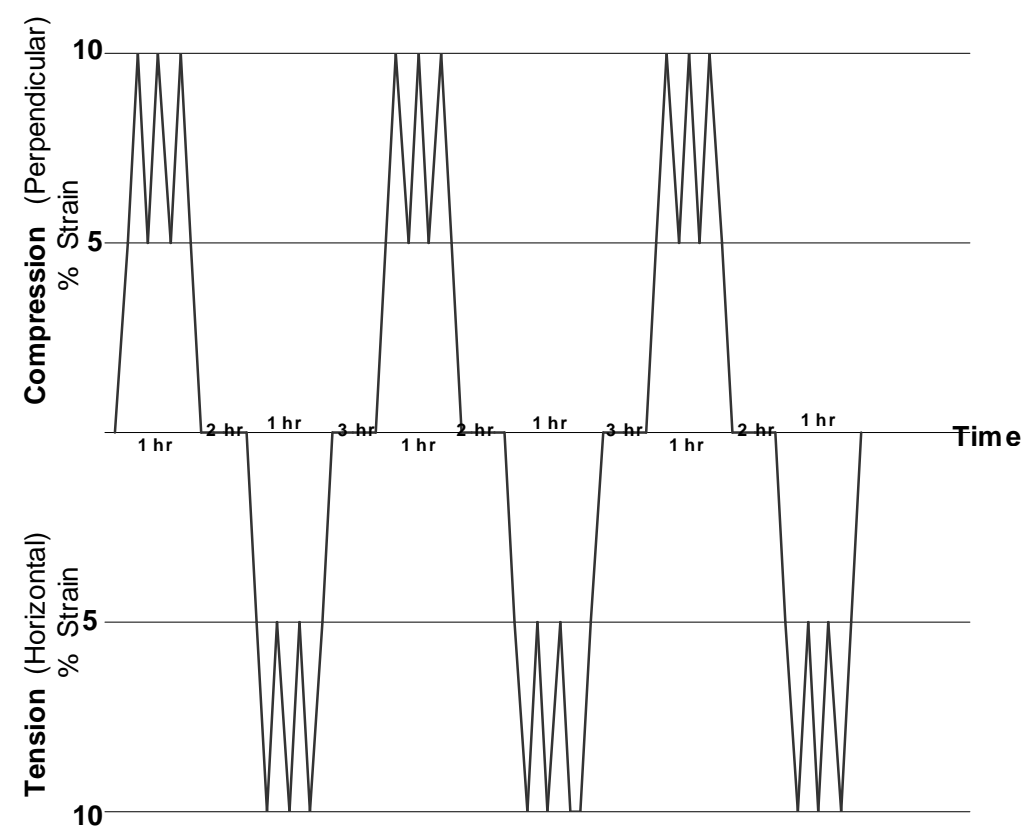

Figure 2.8 Schematic of the stimulation algorithm for stimulation 2 at $10 \%$ total strain stimulation with $5 \%$ offset and $5 \%$ cyclic strain for both compression and tension.

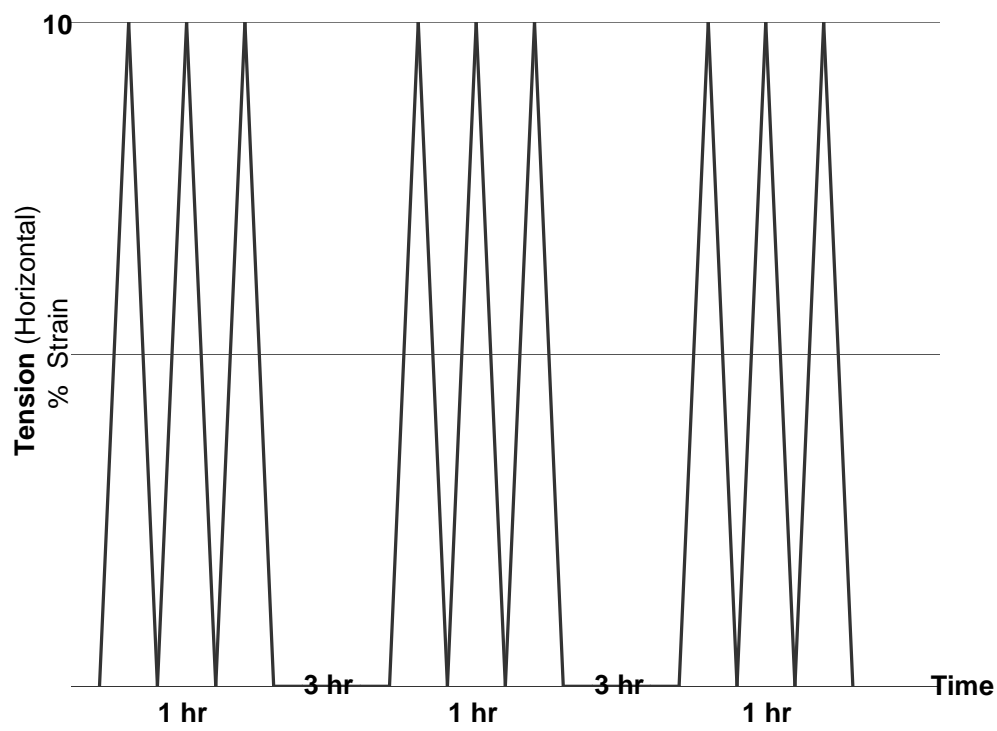

Figure 2.9 Schematic of the stimulation algorithm for stimulation 3 at $10 \%$ cyclic strain for tension only. 
During compression, tension grips held the construct horizontally in place, however there was no additional confinement during tension. Displacement was applied three times daily for 1 hr each with $1 \mathrm{hr}$ between each cycle and $2 \mathrm{hr}$ rest before the second and third set for a total of 2 weeks of stimulation. Loading regimens were chosen for this study based on previous stimulation data preformed in our laboratory on cartilage tissue constructs, in which we found applying $10 \%$ compression or tension resulted in favorable changes without damaging the construct in our bioreactor. Further, loading the construct 3 times daily resulted in the most favorable outcome compared to once and twice daily (data not published). Finally, an offset algorithm was added since previous studies have indicated a benefit with offset loading and application of compression ${ }^{46}$. Upon completion of stimulation, constructs were removed and evaluated by one of the following methods.

\subsection{HISTOLOGY}

Constructs were cut in half along the direction of tension and one half was fixed with $10 \%$ buffered formalin (the other half was used for SEM) for 15 minutes followed by 3 washes with PBS. The constructs were then placed in $20 \%$ sucrose in distilled water and the solution was allowed to infiltrate for several minutes. Sections were placed in Tissue-Tek OCT freezing media (Andwin Scientific, Tryon, NC) in an embedding mold cups for cryostat sectioning and frozen to $20{ }^{\circ} \mathrm{C}$ for at least $24 \mathrm{hr}$. The frozen blocks were removed from the mold and fixed on the sectioning post within a Microm HM500M cryostat (Thermo Fisher Scientific Inc, Waltham, MA). The constructs were sectioned representing the internal edge and the internal plane with the direction of tensile noted at 8 or 10 microns. The cuts were stained for histological evaluation of the presence of proteoglycans using the Alcian blue stain kit (Poly Scientific, Bay 
Shore, NY). Stained slides were viewed and photographed on an Eclipse TE200 microscope (Nikon, Melville, NY). Images were taken of the tissue samples using a DageMTI XLV 185 camera (Dage Technologies, Michigan City, IN) at a 10X magnification and processed using Exponent software (Dage Technologies, Michigan City, IN). Qualitative gross visual comparisons were made between constructs in static culture at Day 0 and stimulated cultures for construct structure and stain color.

\subsection{SCANNING ELECTRON MICROSCOPY}

Two Day 0 constructs and 2 samples from the 3 stimulation groups were evaluated using scanning electron microscopy (SEM). Constructs were first rinsed in $0.1 \mathrm{M}$ sodium cacodylate buffer for one wash followed by fixation in $2 \%$ Glutaraldehyde in $0.1 \mathrm{M}$ sodium cacodylate buffer for 10 minutes. The constructs were removed and stored in $0.1 \mathrm{M}$ sodium cacodylate buffer prior to sectioning. The constructs were sectioned to show the interior edge, interior plane, and edge perpendicular to tension and fixed to the SEM mounting post by the Virginia Commonwealth University Department of Anatomy and Neurobiology Microscope Facility. Samples were sputter coated with gold by an EMS 550x sputter coater (Electron Microscopy Sciences, Hatfield, PA) and viewed in a Zeiss EVO 50 XVP (Carl Zeiss MicroImaging, Inc., Thornwood, NY) scanning electron microscope. Zeiss SmartSEM® software (Carl Zeiss MicroImaging, Inc., Thornwood, NY) captured the images in digital photos. Qualitative visual comparisons were made between the Day 0 and the stimulation group samples for cell and matrix content along with morphology. 


\subsection{CELL ALIGNMENT}

Construct sections used for histological analysis were also used for determination of cell alignment. After the above-mentioned sectioning and staining process, slides were viewed under an Eclipse TE200 microscope (Nikon, Melville, NY). Images were taken of the tissue samples using a DageMTI XLV 185 camera (Dage Technologies, Michigan City, IN) at a 10X magnification and processed using Exponent software (Dage Technologies, Michigan City, IN). Each specimen had 14 pictures taken to evaluate of the cell angle in relation to the direction of tensile stimulation, 7 pictures of the cut parallel to the surface and 7 pictures from the side view cut. Images were processed using ImageJ (NIH freeware) software to evaluate for cell alignment. Briefly, ImageJ was calibrated to set scale for measurements, measurements were set to analyze area and perimeter; each cell was individually outlined with the free-hand tool and measured ${ }^{74}$. Results showed the cell area, perimeter, major \& minor axes, and the angle of the axis from the direction of tension. Total angle of each cell from the direction of tension application was determined for each specimen (approximately 670 to 770 total cells evaluated for each group) and averaged. Comparisons were made between the Day 0 samples and three different stimulation groups.

\subsection{INDENTATION TESTING}

For indentation and tensile testing, some of the constructs were cut in half with part of the construct used for histology or SEM and the remaining for mechanical evaluation. To evaluate the mechanical properties, indentation testing, which involves application of a load to the construct with the change in deformation measured over time, was performed using a customdesigned indentation tester on both Day 0 constructs and mechanically stimulated specimens. A 
constant load was applied to the construct while the equipment recorded the instantaneous and time-dependent creep. Samples were placed inside a tissue well on a central platform and a round steel washer was used to keep the tissue-engineered construct in place. The indenter tip was placed on the surface of the construct and a $1 \mathrm{gm}$ preload was applied for 10 minutes and then a $1 \mathrm{gm}$ load was applied. A PowerMAC (Apple, Cupertino, CA) computer with LabVIEW (National Instruments, Corp., Austin, TX) software recording the instantaneous and timedependent creep values. The load was applied for a 50 minute time period to allow the tissueengineered construct to come to equilibrium. The data was entered in to a curve-fitting program based on the biphasic theory ${ }^{75,76}$ that gives values for aggregate modulus, permeability, and Poisson's ratio for comparison between the Day 0 and stimulated constructs ( $\mathrm{N}=9$ for all groups).

\subsection{TENSILE TESTING}

After completion of indentation testing, nine constructs ( $\mathrm{N}=9$ for all groups) from each stimulation algorithm were tensile tested on a Bionix tensile testing device (MTS Systems, Eden Prairie, MN) with data acquisition software Testworks 4.0.6A (MTS Systems, Eden Prairie, $\mathrm{MN}$ ). Application of tensile loading was performed on dog-bone cut shaped specimens placed in grips and loaded at $5 \mathrm{~mm} / \mathrm{min}$. Thickness was measured with a Mitutoyo digital micrometer (Mitutoyo, Kawasaki, Japan) and width and gage length was measure with Mitutoyo digital calipers (Mitutoyo, Kawasaki, Japan). Plots of load versus extension and stress versus strain were used to determine peak load, peak stress, and calculate modulus for each of the tested samples. The linear portion between $90-50 \%$ of the peak stress of the stress-strain curve was used to calculate modulus. 


\subsection{WESTERN BLOT ANALYSIS}

For protein extraction, performed on three tissue constructs at Day 0 and after mechanical stimulation for all three stimulation algorithms ( $\mathrm{N}=3$ for all groups), constructs were rinsed in PBS and spun for $1 \mathrm{~min}$ at $100 \mathrm{RPM}$ to remove the majority of the media present in the scaffold. Constructs were cut with sterilized scissors into very small pieces, place on ice, and treated with lysis buffer consisting of Mammalian Protein Extraction Reagent (Thermo Fisher Scientific, Waltham, MA), Halt Protease Inhibitor (Thermo Fisher Scientific, Waltham, MA), and EDTA (ethylenediaminetetraacetic acid) (Thermo Fisher Scientific, Waltham, MA). Mixture was homogenized using a PowerGen Model 125 homogenizer (Fisher Scientific, Pittsburgh, PA). Once constructs were completely homogenized, mixture was removed and placed in $1.5 \mathrm{ml}$ eppendorf tubes (Eppendorf, Westbury, NY), then centrifuged at 14000 RPM for 10 minutes at $4^{0} \mathrm{C}$ in a Centrifuge Model 5402 (Eppendorf, Westbury, NY).

The supernatant containing the protein fraction was removed and placed in a $1.5 \mathrm{ml}$ eppendorf tube and stored at $-70^{\circ} \mathrm{C}$ until further analysis. Protein concentration was analyzed with a standard curve of serial diluted samples and Bradford dye (Bio-Rad Laboratories, Hercules, CA) and SmartSpec 3000 (Bio-Rad Laboratories, Hercules, CA). Human cartilage was used as a control group undergoing the same procedure as above with the exception that before homogenization, the cartilage was flash frozen with liquid nitrogen and crushed up with a mortar and pestle. Once the concentration was determined, the amount of sample with loading dye was added to one of the wells in the gel. A $10 \%$ acrylamide gel containing distilled water, $10 \%$ of $30 \%$ acrylamide/Bis (Bio-Rad Laboratories, Hercules, CA), 1.5 Tris pH 8.8 (Bio-Rad Laboratories, Hercules, CA), SDS (Sodium Dodecyl Sulfate) (Bio-Rad Laboratories, Hercules, CA), APS (Ammonium Persulfate) (Bio-Rad Laboratories, Hercules, CA) and TEMED 
(tetramethylethylene diamine) (National Diagnostic USA, Atlanta, GA) was used with a 5\% acrylamide stacking gel on top.

In addition to the protein samples, the gel was loaded with two-color protein molecular weight marker 928-40001 (Li-Cor, Lincoln, NE) and the electrophoresis Model 1000 (Bio-Rad Laboratories, Hercules, CA) set to run for 2 hours at 100 Volts. After completion of the 2 hour time period, the gel was removed and set up for protein transfer. A Scotchbrite pad (3M, Saint Paul, MN), western blotting filter paper (Thermo Fisher Scientific, Waltham, MA), the gel with the marker side down, Transblot Transfer Medium Pure Nitrocellulose Membrane (Bio-Rad Laboratories, Hercules, CA), another piece of filter paper, followed by a Scotchbrite pad were loaded into the cassette, placed in the transfer chamber and transfer buffer (kept at $4{ }^{0} \mathrm{C}$ containing 10X Tris/Glycine (Bio-Rad Laboratories, Hercules, CA), methanol, and distilled water was added. The chamber was connected to the electrophoresis and run at 100 Volts for 2 hours.

Upon completion, the membrane and gel were carefully removed and placed in Tris Buffered Saline (Bio-Rad Laboratories, Hercules, CA) and Tween-20 (Fisher Scientific, Pittsburgh, PA) within a plastic container. The gel was slowly removed from the membrane and the solution was poured out. Odyssey blocking buffer 927-40000 (Li-Cor, Lincoln, NE) was slowly added and the container was placed on a shaker for 10 minutes. After 10 minutes, the anti-aggrecan MAB19310 (Millipore, Billerica, MA) was added to the blocking solution and the container was placed in a refrigerator at $4{ }^{\circ} \mathrm{C}$ on a shaker Model 260350 Rocker II (Boekel Scientific, Feasterville, PA) overnight.

The following day the antibody solution was removed and after four 10 minute rinses with TBST on a shaker GeneMate 0S350 Orbital Shaker (GeneMate Technologies Pvt. Ltd. New 
Delhi, India), the secondary antibody Goat anti-mouse IR-dye (Li-Cor, Lincoln, NE) was added to a $1 \%$ milk TBST solution and placed in the membrane container for 2 hour. After the secondary antibody was removed, the membrane was rinsed four times with TBST for 5 minutes each. The membrane was then placed on an Odyssey Infrared Imaging System (Li-Cor, Lincoln, NE) scanner and the image scanned with Li-Cor Application Software Version 2.1 (Li-Cor, Lincoln, NE) on an Optiplex GX620 (Dell, Round Rock, TX) Computer. The captured photo was saved as a tiff file and analyzed for the protein content for comparison between the Day 0 and stimulated constructs.

Following scanning of the membrane, Odyssey blocking buffer was again added to the membrane with antibody to GAPDH (Santa Cruz Biotechnology, Santa Cruz, CA) added for comparison as a loading control and the same procedures as used for anti-aggrecan antibody were used. Tiff files were converted using Photoshop (Adobe, Santa Jose, CA) to a high quality jpeg and analyzed using Alphaease software (Cell Biosciences, Santa Clara, CA).

\subsection{STATISTICAL ANALYSIS}

All statistical analysis was performed with SAS (Cary, NC) statistical software. Alignment, indentation, tensile, and western blot values were analyzed for each stimulation algorithm and Day 0 constructs. Statistical comparisons were made via a one-way analysis of variance (ANOVA) and a Tukey-Kramer multiple comparison test (alpha=0.05) to evaluate the effects of stimulation algorithm on the construct properties. 


\section{CHAPTER 3 RESULTS}

\subsection{BRIEF OVERVIEW}

To develop a cartilage tissue engineered construct with properties similar to the superficial tangential zone with matrix content and arrangement, three stimulation algorithms were evaluated and compared to Day 0 constructs. Evaluation of the properties was completed using histology, scanning electron microscopy (SEM), indentation testing, tensile testing, cellular alignment, and western blot analysis for aggrecan with the results reported below.

For clarification, the locations within each construct for histological and SEM sections are diagramed below in Figures 3.1. In the diagram, a single-headed red arrow indicates the direction of compression and double-headed for tension. For histology, Figure 3.1 depicts an internal edge section (C) and an internal surface section (D). All histology sections were taken from within the scaffold or construct. SEM sections were made from an internal edge section (C), internal surface sections (D), and perpendicular surface sections (E) (Figure 3.1). Additionally, some SEM surface images were taken of the external surface as indicated by (B) in Figures 3.1.
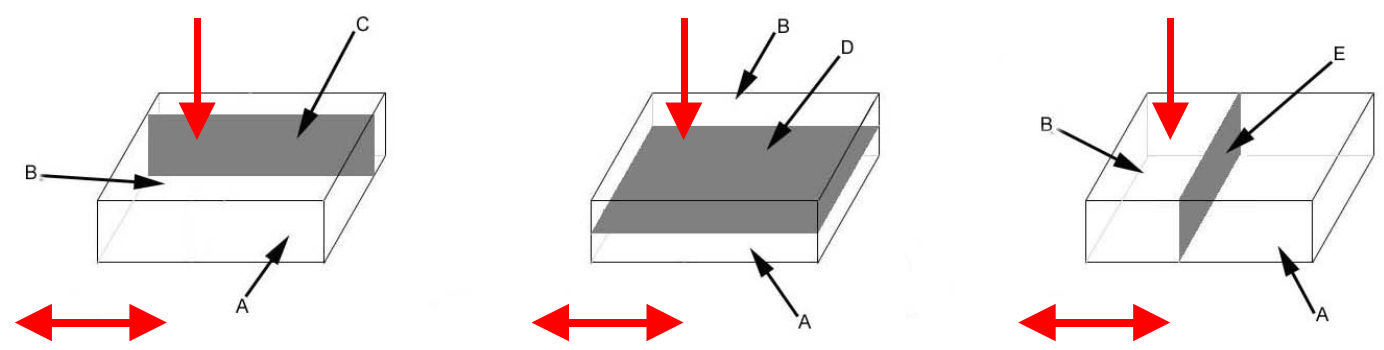

Figure 3.1 Schematic diagram of the locations within constructs for histological and SEM sections: (A) external edge parallel to the tension direction, (B) external surface in the direction of tension, (C) internal edge in the direction of tension parallel to the external edge, (D) internal plane section parallel to the surface in the direction of tension, and (E) internal edge perpendicular to the direction of tension (direction of compression is indicated with single-headed red arrow and tension is indicated with double-headed arrow). 


\subsection{HISTOLOGY}

\section{$\underline{\text { Scaffold Only }}$}

Evaluation of Collagen type I scaffold alone stained with alcian blue stain show a pink coloration of the collagen. Figures 3.2 shows two different sections of the scaffold, where both exhibit similar pink coloration. Figure 3.2(A) is an internal edge section (refer to Figure 3.1C) of the scaffold and Figure 3.2(B) is an interior plane parallel (refer to Figure 3.1D) to the surface section. Both sections illustrate the porous nature of the scaffold, with large areas of open spacing, where no scaffold material was present, and no blue coloration due to the absence of mucopolysaccharides (proteoglycans).
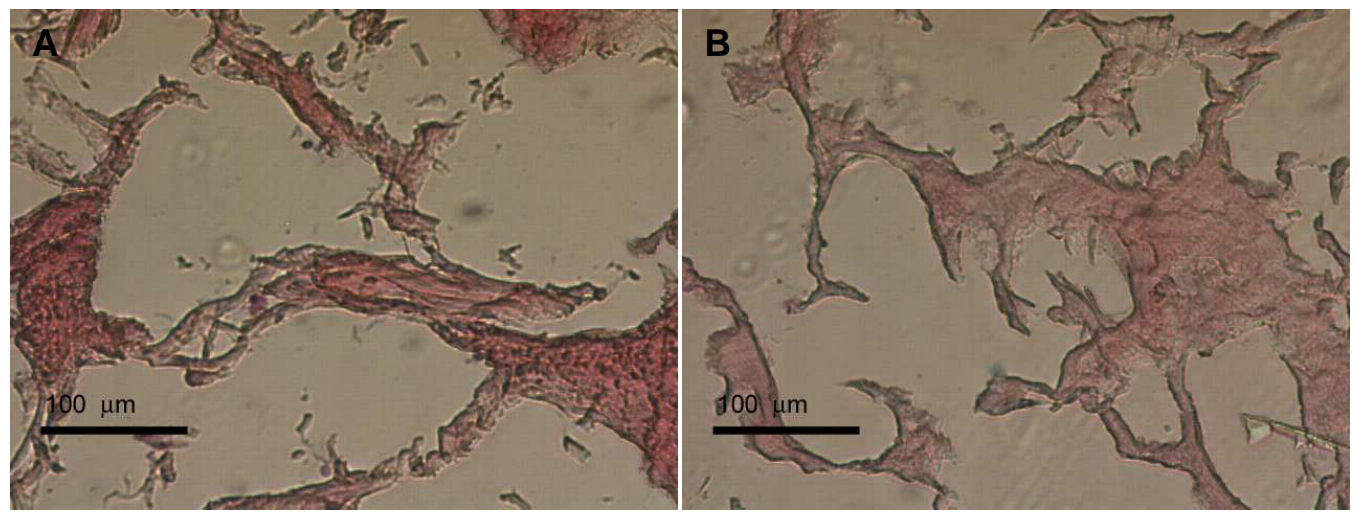

Figure 3.2 Histology picture of a Kensey Nash Collagen type 1 scaffold of an internal edge section (A) and an interior plane parallel to surface section (B) (scale bar is $100 \mu \mathrm{m}$ ).

$\underline{\operatorname{Day} O}$

In the Day 0 constructs, which were kept in static culture for 7 days prior to fixation, there was much less void space within the scaffold, where it exhibited matrix production in addition to the initial scaffold as illustrated by a decrease in the open pore area within the constructs (Figure 3.3). The seeding method was sufficient as the cells (showing a darkish pink stain) are seen throughout the construct, as well as on the surface of the scaffold. Additionally, the cells (indicated by white circles) no longer showed the stellate formation that they do in 
monolayer, but more of a rounded, spheroid shape, exhibiting a characteristic similar to chondrocytes. Figures 3.3(A) and 3.3(C) are of an internal edge section (refer to Figure 3.1C) of the construct and show the distribution of cells throughout the thickness of the collagen scaffold. Some cells appeared to be elongated in their shape, but not in any particular direction that was readily apparent. The cells in the constructs produced GAGs, as illustrated by the blue staining (indicated by white arrows), although there was more abundance of GAGs in the edge sections as compared to their surface counterparts. The interior plane sections (refer to Figure 3.1D) of both constructs (Figures 3.3(B) and 3.3(D)) showed very little blue staining, indicating not much GAG production deeper within the construct. Additional pictures of the Day 0 constructs can be viewed in Appendix A.

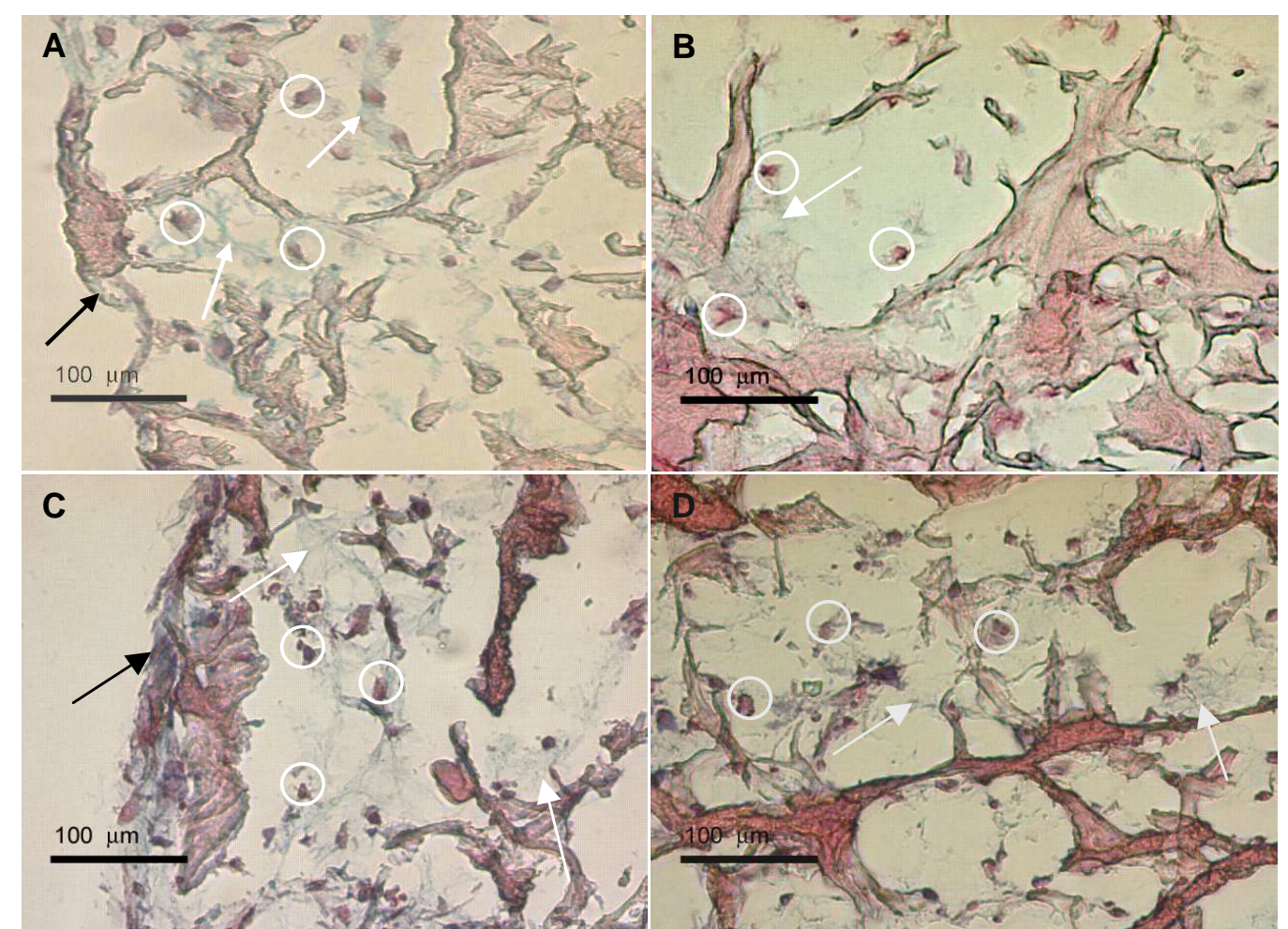

Figure 3.3 Construct 155A (A and B) and Construct 186D (C and D), Day 0 with no mechanical stimulation. Shown are edge sections with black arrows indicating the exterior surface (A and $\mathrm{C}$ ) and a plane parallel to the surface (B and D). Cells are identified in the images with white circles and blue GAG staining with white arrows (scale bar is $100 \mu \mathrm{m})$. 


\section{Compression and tension without offset}

The constructs for compression and tension without offset were pre-cultured for 7 days prior to placement in the bioreactor, then stimulated for 2 weeks with both compression and tension without offset stimulation. In these constructs, cells (indicated by white circles) stained a darkish-pink and were seen throughout the thickness of the constructs (Figure 3.4), similar to the Day 0 constructs. Cell conformation was more rounded with some cells even elongated, but there did not seem to be any direction of preferential alignment from solely gross visual evaluation. Blue staining of GAGs (indicated by white arrows) was visible in the edge sections (Figures 3.4(A) and (C)) as well as the interior plane sections (Figure 3.4(B) and (D)), which was not the case in Day 0 constructs. Since the interior plane sections showed more interior of the construct, there were more GAGs present further within the construct than the Day 0 constructs. As in the Day 0 constructs, the constructs without offset exhibited production of extracellular matrix components in addition to the initial collagen scaffold, which was readily seen by a decrease in the porous areas present. There was more GAG staining present in constructs with compression and tension without offset than tension alone, indicating compression facilitated an increase in GAG expression. Additional pictures of the compression and tension with no offset constructs can be viewed in Appendix A. 


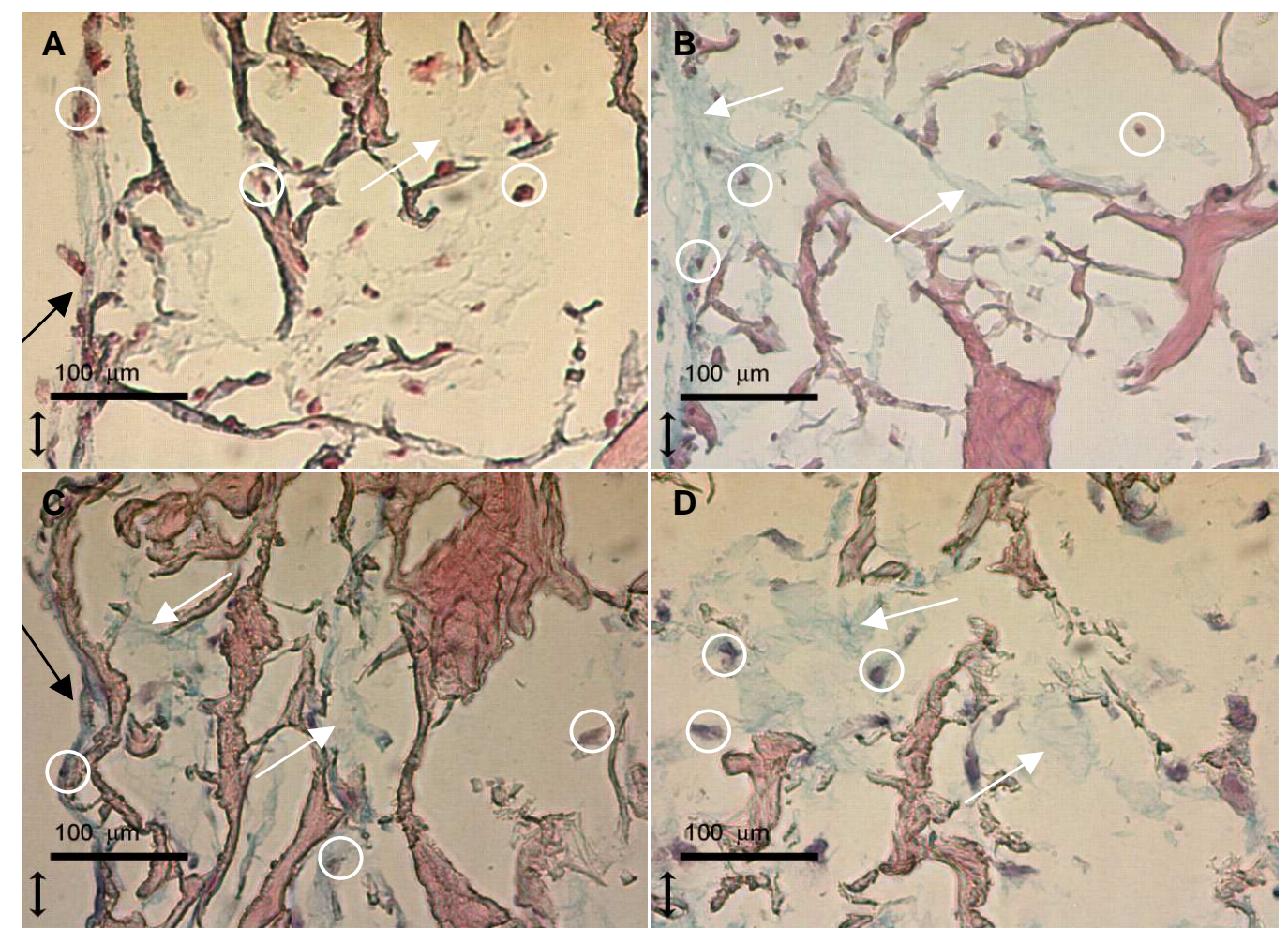

Figure 3.4 Construct 163A (A and B) and Construct 209A (C and D) with both compression and tension without offset. Shown are edge sections, with black arrows indicating the exterior surface (A and C), and interior plane sections (B and D). Cells are identified in the images with white circles and blue GAG staining with white arrows (scale bar is $100 \mu \mathrm{m}$ ).

\section{Compression and tension with offset}

Following the 7-day pre-culture period, the constructs for compression and tension with an offset were inserted in the bioreactor and stimulated for 2 weeks by compression with an offset and tension with an offset. Dark pink stained cells (indicated by white circles) were permeated throughout the offset stimulated constructs and were in a round morphological conformation (Figure 3.5). Additionally, the cells were elongated but with no discernable configuration of a preferential arrangement. The edge sections showed a large amount of blue staining near and around the interior plane (the surface of the edge section was indicated with a black arrow) (Figure 3.5(A) and (C)) indicating deposition of GAGs (indicated by white arrows). Additionally, the interior plane sections in both constructs (Figure 3.5(B) and 3.5(D)), showed 
blue staining similar in amount and arrangement to the constructs without offset. As in the Day 0 and constructs without offset stimulation, further matrix production was readily apparent when compared to scaffold alone. Similar to the without offset constructs, constructs stimulated with both compression and tension with an offset had an increased amount of GAG present than tension alone, which suggested there was a qualitative increase when compression was applied in addition to tension, that didn't appear in tension alone. Additional pictures of the compression and tension with no offset constructs can be viewed in Appendix A.

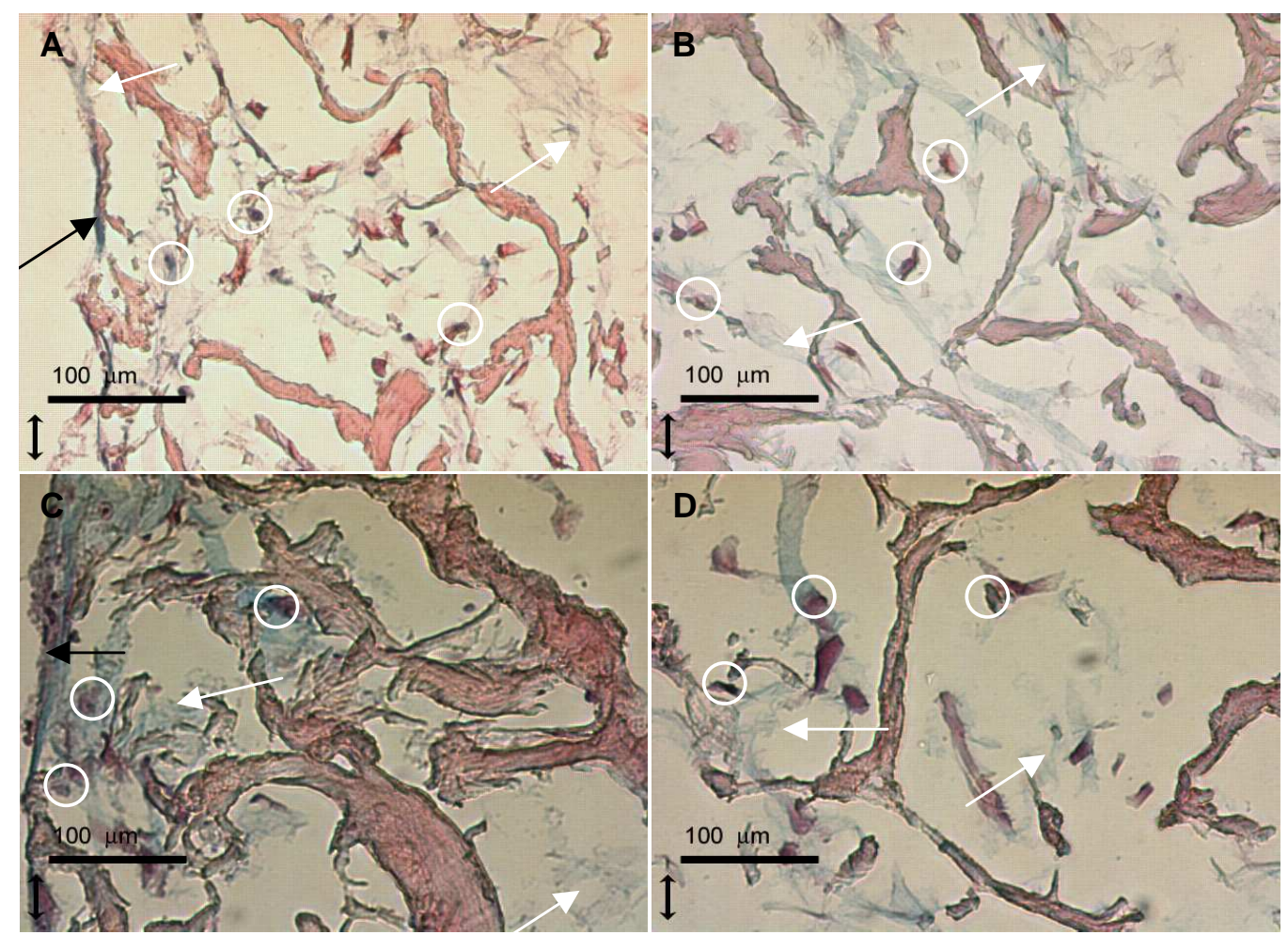

Figure 3.5 Construct 186B (A and B) and Construct 188B (C and D) with both compression and tension with an offset, illustrating edge sections, with black arrows indicating the exterior surface (A and C) and interior plane sections (B and D). Cells are identified in the images with white circles and blue GAG staining with white arrows (scale bar is $100 \mu \mathrm{m}$ ).

\section{$\underline{\text { Tension Only }}$}

Tension only constructs were stimulated for 2 weeks with tension with no offset stimulation, following the 7-day pre-culture period. Throughout the construct thickness, dark 
pink stained cells (indicated by white circles) were seen (Figure 3.6), however, there were less visible in construct 197B (Figure 3.6(A) and (B)). The tension stimulated constructs had cells with a rounded morphology in both the edge and interior plane sections, with some cells exhibiting elongation. Alcian blue staining for GAGs was seen at the surface (indicated by black arrows) in the edge section of construct 199A, but very little was seen in construct 197B (Figure 3.6(A) and (C)). However, in the interior plane sections for both constructs, there is a large amount of staining for GAGs, comparable to the staining seen in the other stimulated constructs (Figure 3.6(B) and (D)). Tension only constructs also had noticeable extracellular matrix deposition in addition to the original scaffold. Further pictures of the tension only constructs can be viewed in Appendix A.

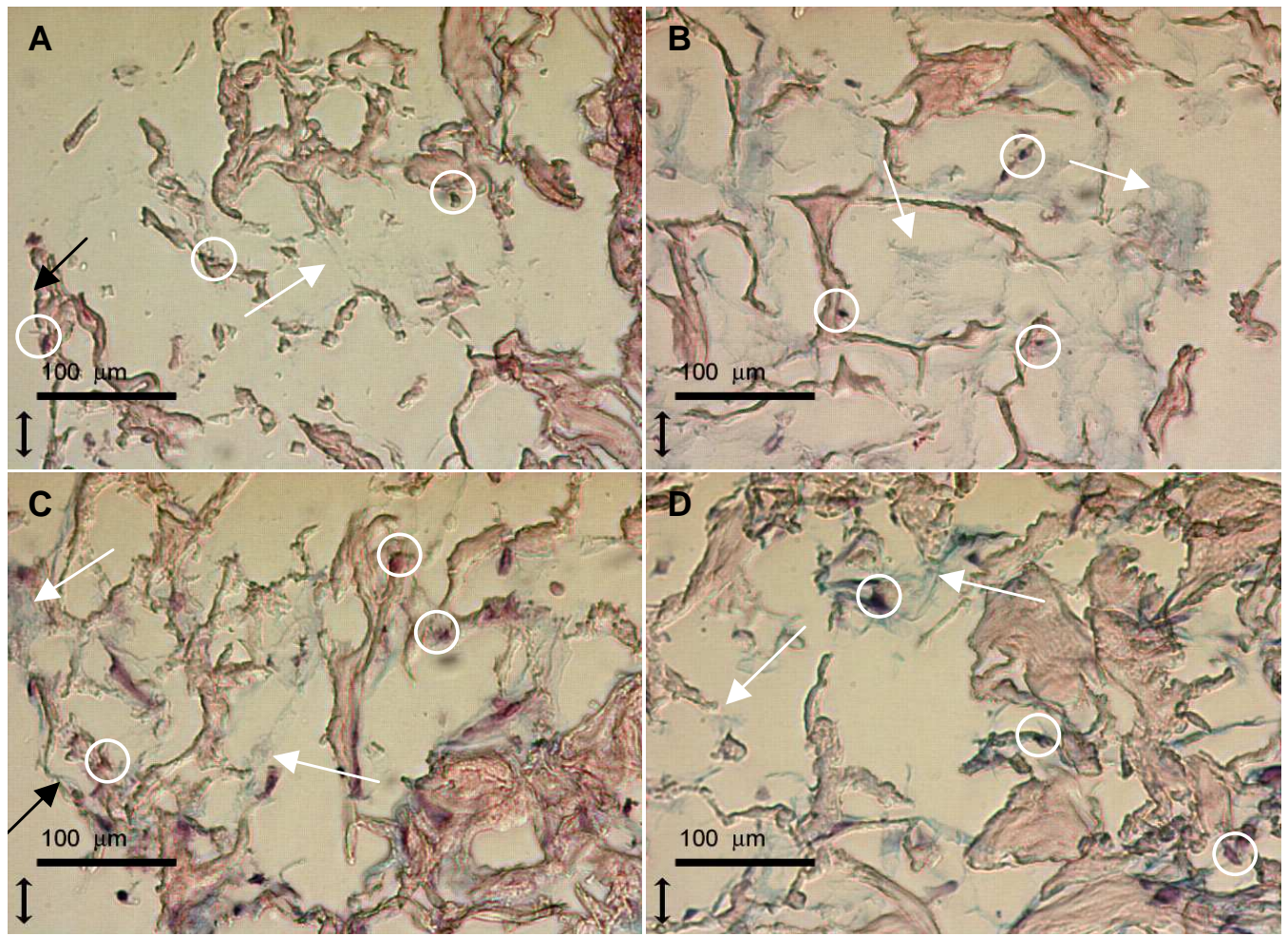

Figure 3.6 Construct 197B (A and B) and Construct 199A (C and D) with tension only, illustrating edge sections, with black arrows indicating the exterior surface (A and $\mathrm{C}$ ) and interior plane sections (B and D). Cells are identified in the images with white circles and blue GAG staining with white arrows (scale bar is $100 \mu \mathrm{m}$ ). 


\subsection{SCANNING ELECTRON MICROSCOPY}

\section{Scaffold Only}

The Scanning electron microscopy (SEM) images of the Kensey Nash Collagen type I scaffolds showed a scaffold structure with a high porosity (Figure 3.7). Both the edge section (Figure 3.7(A)) and exterior surface section (Figure 3.7 (B)) had pores throughout their depth, which appeared to vary in size anywhere from 20 to 200 microns. The collagen type I scaffold exhibited a fibrous structure with a large number of surface areas available for possible cellular attachment. Both internal and external sections of the scaffold were similar in their appearance. Additional pictures of the collagen type I scaffolds can be viewed in Appendix A.

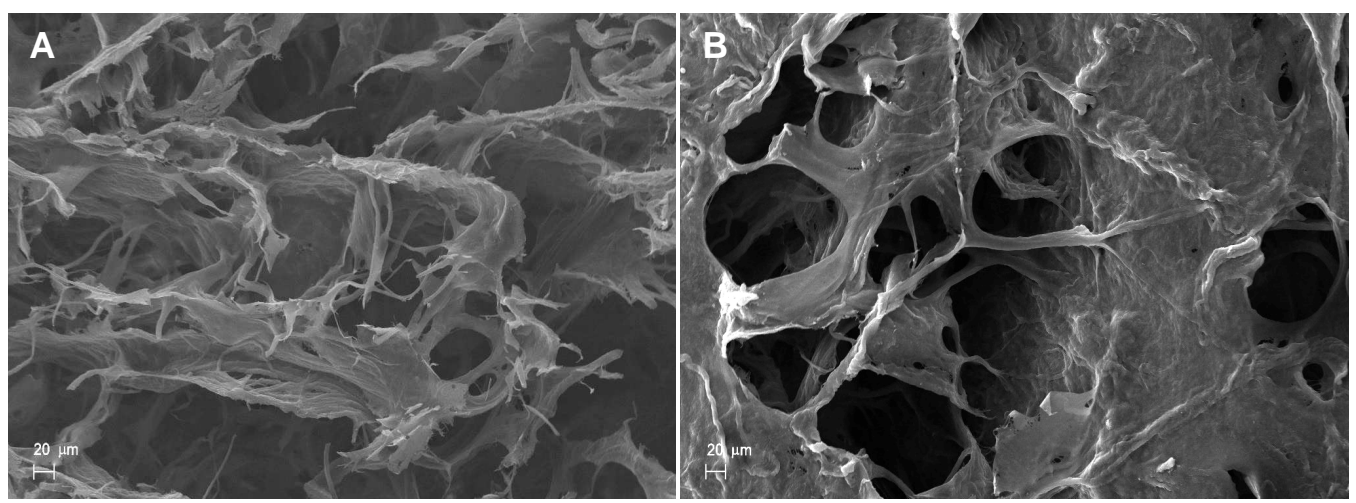

Figure 3.7 Kensey Nash Collagen type I scaffold (A) edge and (B) exterior surface section view (scale bar is 20 $\mu \mathrm{m})$.

$\underline{\text { Day } 0}$

Constructs cultured statically for 7 days exhibited matrix deposition in the pores of the Collagen type I scaffold (Figure 3.8 and 3.9), although there was still some porosity present. There were some visible cells (indicated by white circles) attached to the matrix in the internal edge section views (Figures 3.8(A) and 3.9(A)). Additionally, the placement of the matrix deposition was in a linear arrangement running vertical in Figure 3.8(A) and horizontal in Figure 3.9(A). The internal construct interior plane sections (Figure 3.8(B) and 3.9(B)) also had some 
cells attached with a round morphology and a decreased porosity compared to scaffold alone due to production of matrix materials by the cells. The external surface was completely covered and filled in with matrix material within 7 days of static culture (Figure 3.8(C) and 3.9(C)). Additional SEM images of Day 0 constructs can be viewed in Appendix A.

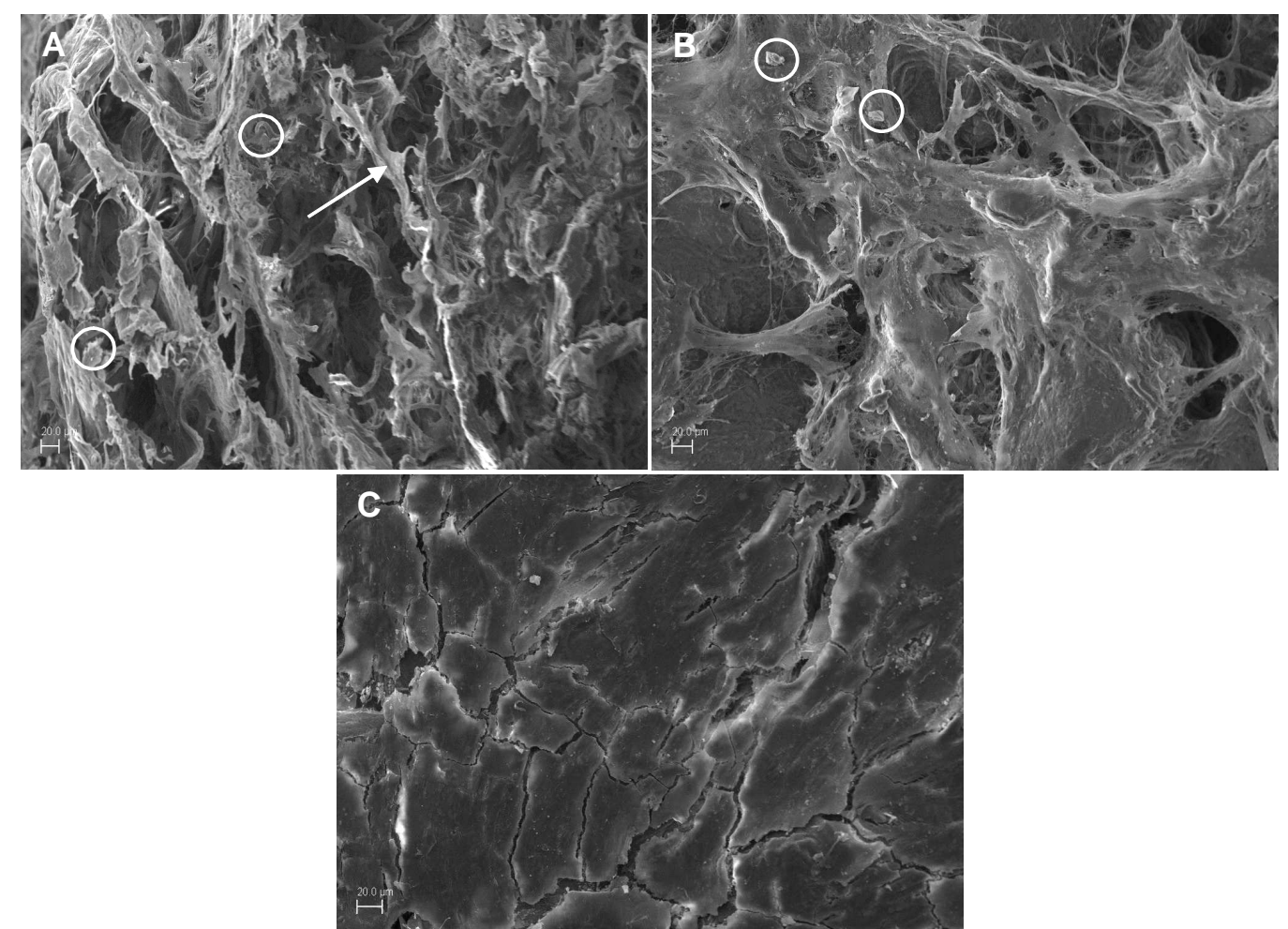

Figure 3.8 Construct 212A with no stimulation, illustrating an (A) internal edge section view, (B) interior plane section parallel to tension, and (C) external surface section (scale bar is $20 \mu \mathrm{m}$ ). White circles indicate cells and a white arrow indicates matrix. 

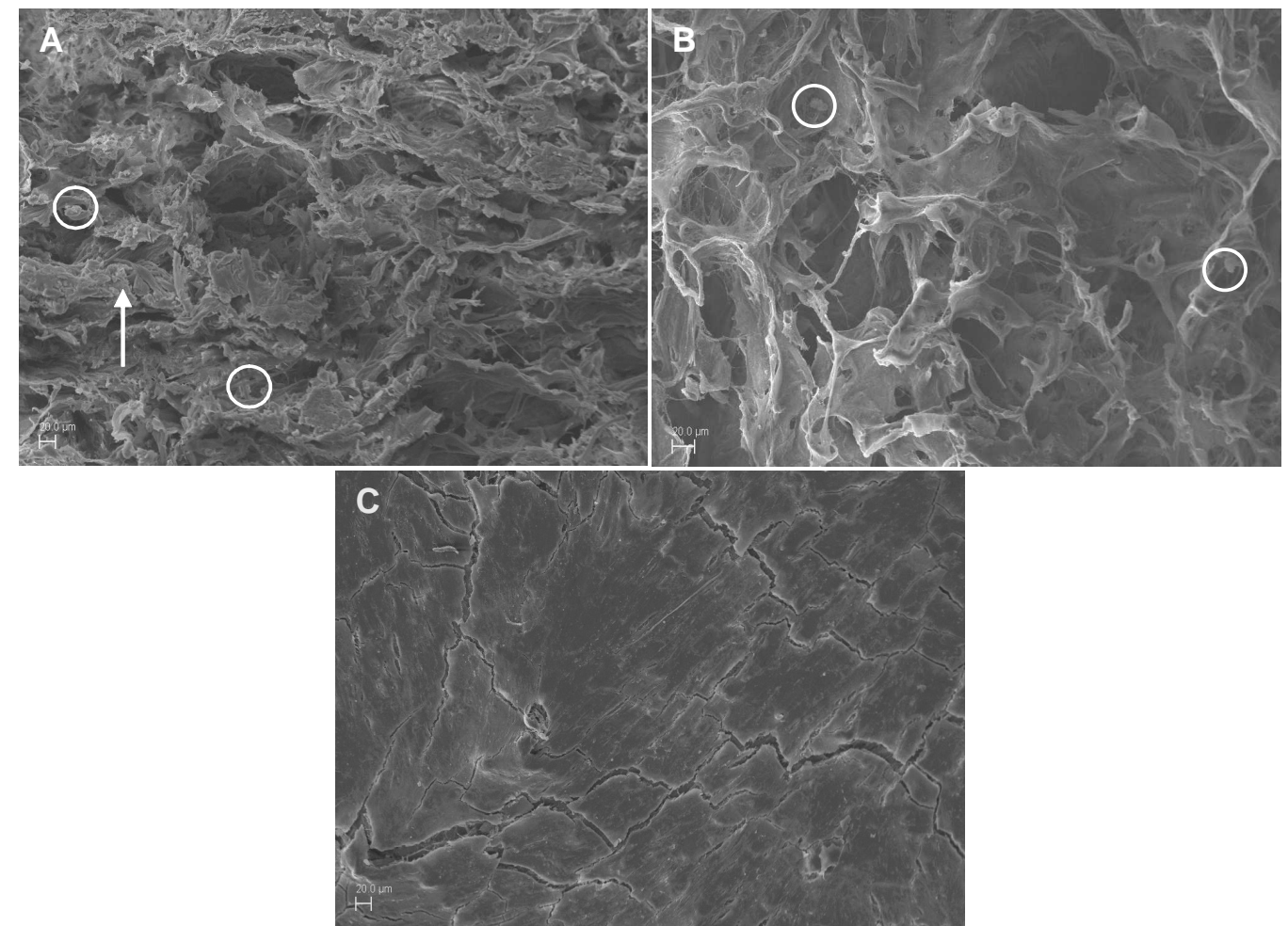

Figure 3.9 Construct 212B with no stimulation, illustrating an (A) internal edge section view, (B) internal parallel to interior plane section, and (C) external surface section (scale bar is $20 \mu \mathrm{m}$ ). White circles indicate cells and a white arrow indicates matrix.

\section{Compression and Tension without offset}

Matrix fibers appeared thicker (indicated by white arrows) than the Day 0 constructs (Figure 3.10 and 3.11) in constructs stimulated with compression and tension without offset stimulation. The internal edge section showed even less porosity than the Day 0 (Figure 3.10(A) and 3.11(A)) and numerous cells were observed (indicated by white circles). Matrix deposition was more organized, rather than randomly placed in the direction of tension, as seen in both the internal edge (Figure 3.10A and 3.11A) and internal interior plane sections (Figure 3.10(B) and 3.11(B)). Internal construct interior plane (Figure 3.10(B) and 3.11(B)) had cells attached with a round morphology and construct 170A (Figure 3.11(B)) showed a greater number of noticeable cells than the Day 0 constructs and construct 166C. Furthermore, both constructs' perpendicular sections (Figure 3.10(C) and 3.11(C)) showed cells exhibiting a round morphology, and much of 
the pores were filled in with thick extracellular matrix (indicated by white arrows). Construct

166C had its greatest number of visible cells in the perpendicular section (Figure 3.10(C)).

Additional SEM images of stimulated constructs with no offset are in Appendix A.
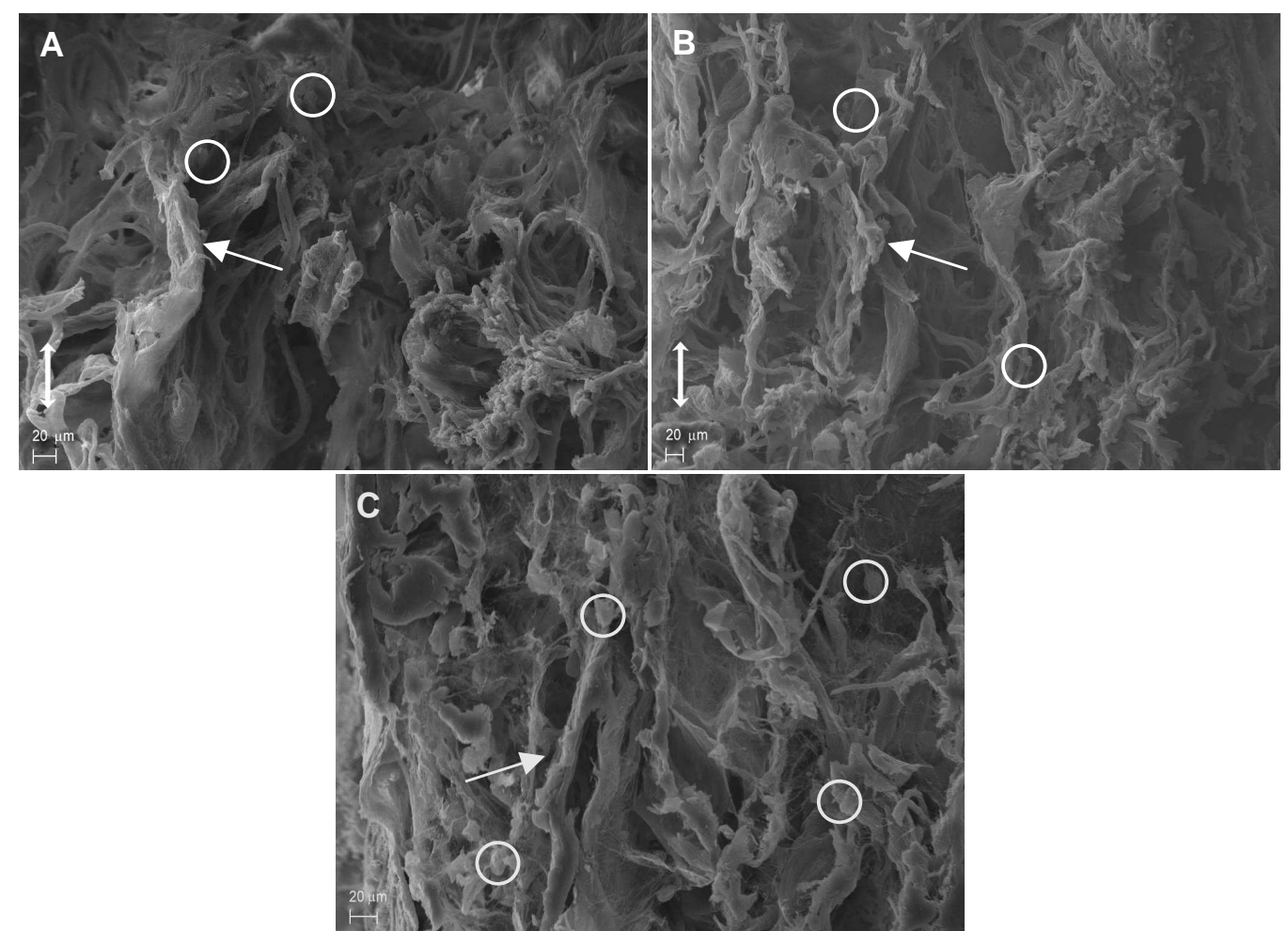

Figure 3.10 Construct 166C with both compression and tension without offset, illustrating an (A) internal edge section view, (B) interior plane section parallel to tension, and (C) internal edge section perpendicular to tension (tension direction is going in to picture). White circles indicate cells, white single-headed arrows indicate matrix, and white double-headed arrows indicate direction of tension application (scale bar is $20 \mu \mathrm{m}$ ). 


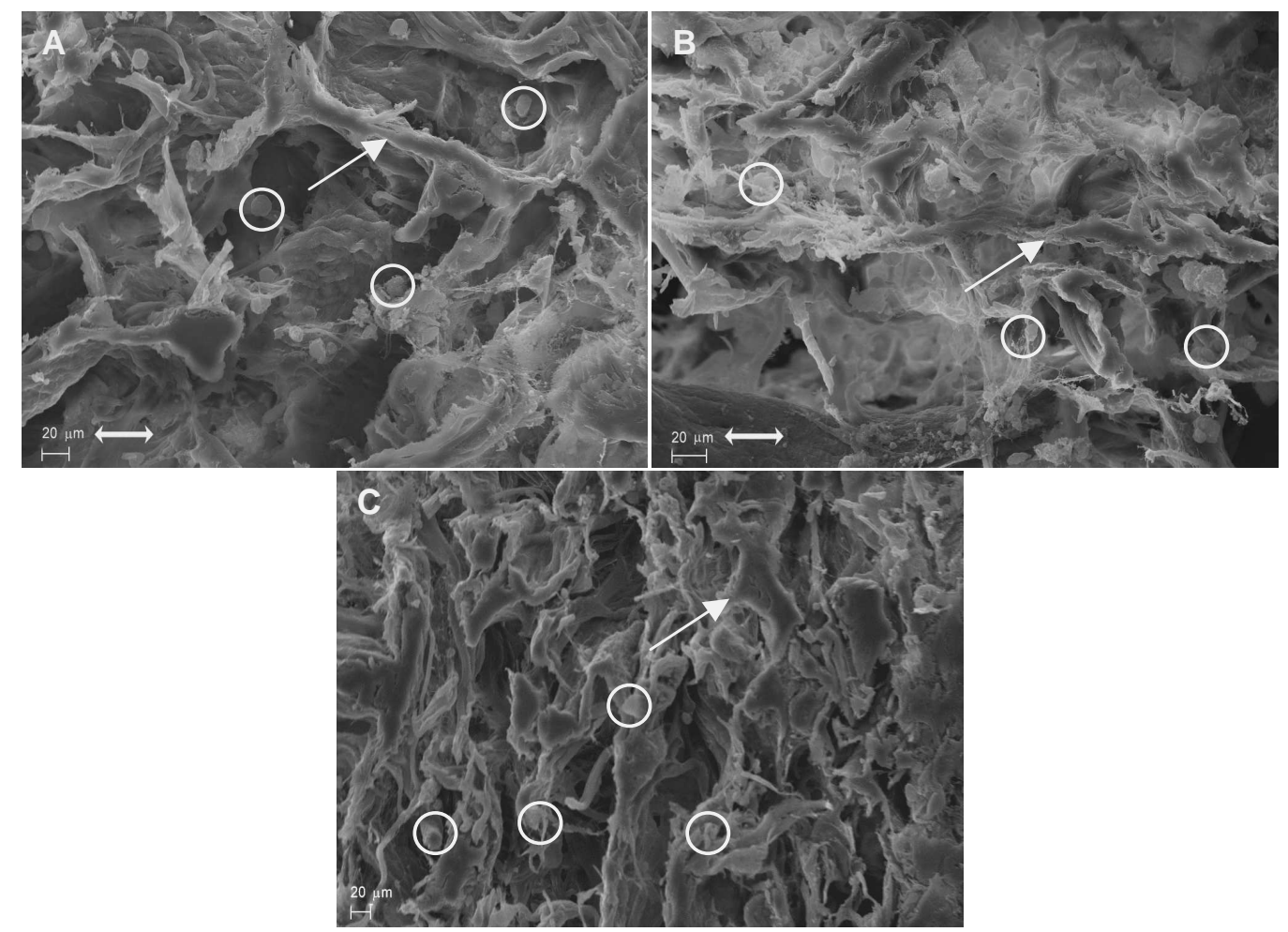

Figure 3.11 Construct 170A with both compression and tension without offset, illustrating an (A) internal edge section view, (B) internal parallel to interior plane section, and (C) internal edge section perpendicular to tension (tension direction is going in to picture). White circles indicate cells, white single-headed arrows indicate matrix, and white double-headed arrows indicate direction of tension application (scale bar is $20 \mu \mathrm{m}$ ).

\section{Compression and tension with offset}

Compression and tension with an offset stimulated constructs revealed matrix fibers (indicated by white arrows) that were thicker than the Day 0 constructs (Figure 3.12 and 3.13), but similar to constructs stimulated with compression and tension without an offset. The internal edge section also showed porosity similar to the constructs stimulated without an offset (Figure 3.12(A) and 3.13(A)) and the amount of visible cells (indicated by white circles) was consistent in each section (Figure 3.12(A, B, C) and 3.13(A, B, C)). Rather than random in appearance, matrix deposition was found to be more ordered in the direction of application of tension seen in both the internal edge (Figure 3.12(A) and 3.13(A)) and interior plane sections (Figure 3.12(B) and 3.13(B)). The internal construct interior plane (Figure 3.12B and 3.13B) had numerous cells 
attached with a round morphology. Furthermore, both construct's perpendicular sections (Figure 3.12(C) and 3.13(C)) had discernible cells, exhibiting a round morphology, and much of the pores were filled in, more so than the constructs stimulated without an offset. More SEM images of stimulated constructs with an offset are in Appendix A.
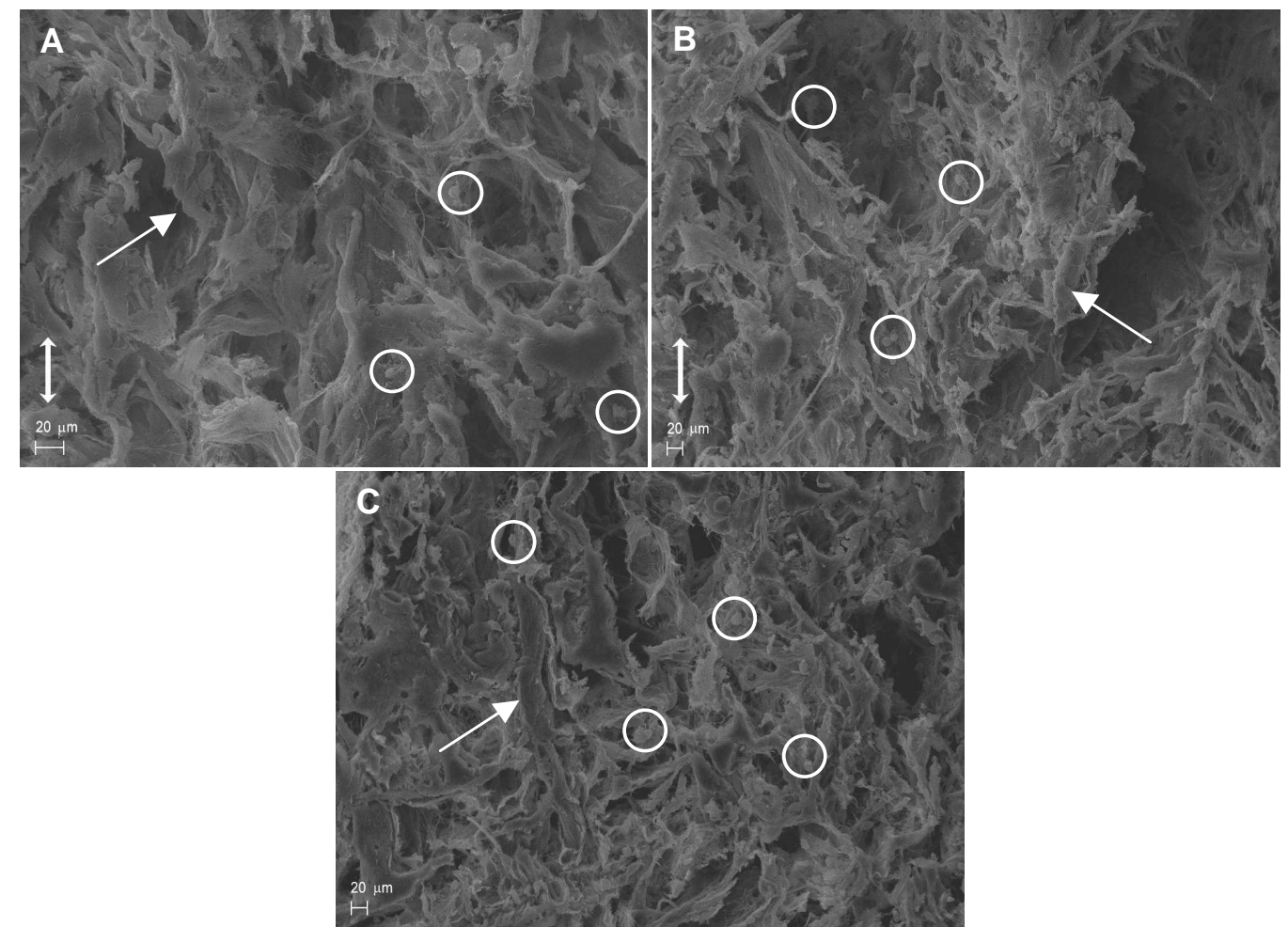

Figure 3.12 Construct 186B with both compression and tension with offset, illustrating an (A) internal edge section view, (B) interior plane section parallel to tension, and (C) internal edge section perpendicular to tension (tension direction is going in to picture). White circles indicate cells, white single-headed arrows indicate matrix, and white double-headed arrows indicate direction of tension application (scale bar is $20 \mu \mathrm{m}$ ). 

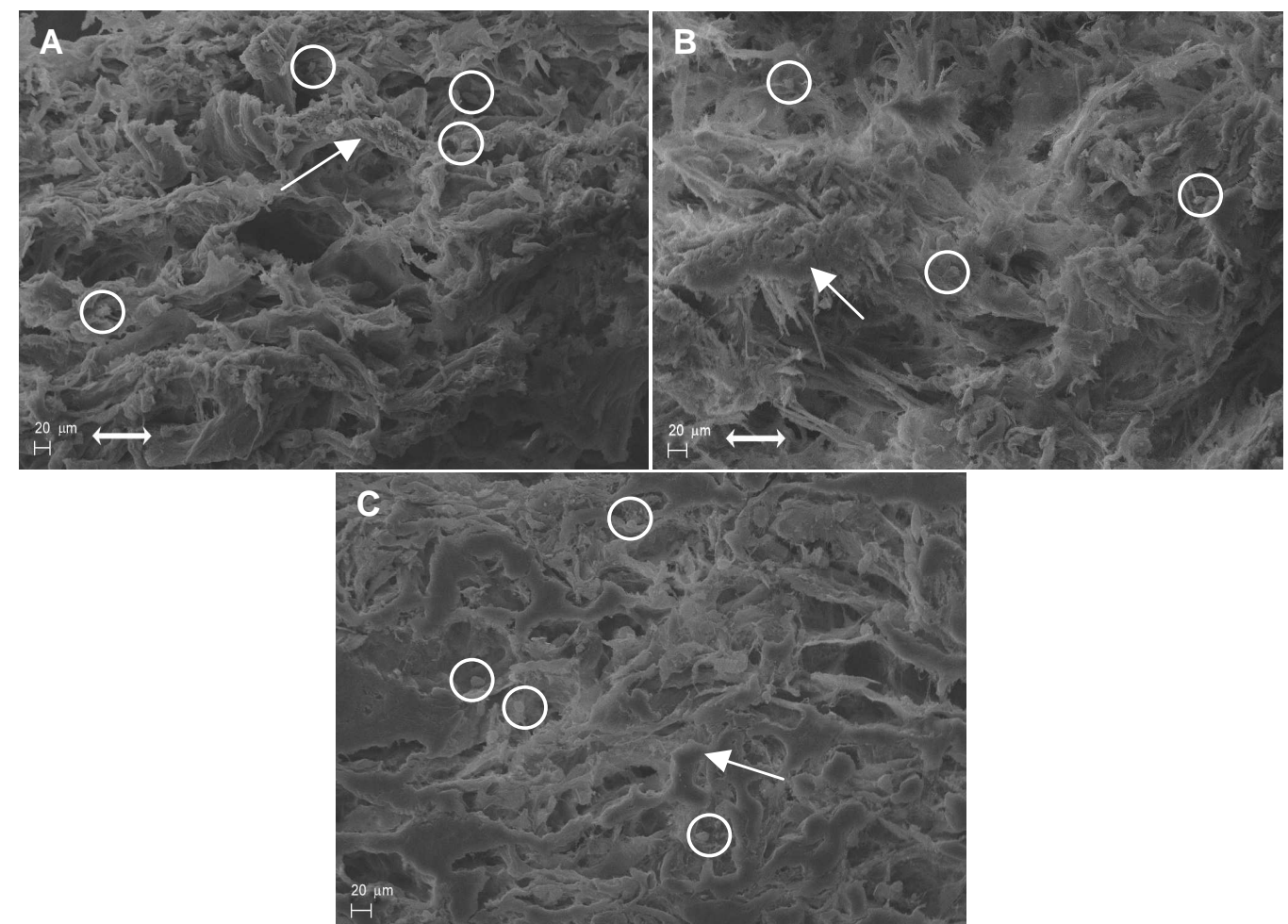

Figure 3.13 Construct 188B with both compression and tension with offset, illustrating an (A) internal edge section view, (B) internal parallel to interior plane section, and (C) internal edge perpendicular to tension (tension direction is going in to picture). White circles indicate cells, white single-headed arrows indicate matrix, and white doubleheaded arrows indicate direction of tension application (scale bar is $20 \mu \mathrm{m}$ ).

\section{$\underline{\text { Tension Only }}$}

Similar to the other stimulation algorithms, fibers of the constructs stimulated with tension only expressed matrix fibers (indicated by white arrows) thicker than the Day 0 constructs (Figure 3.14 and 3.15). Further, the internal edge section showed porosity similar to the other stimulations (Figure 3.14(A) and 3.15(A)). The internal edge section (indicated by white circles) in both constructs exhibited less observable cells than stimulation without offset and with offset. Matrix deposition was not similar to the other stimulation algorithms, the fibers did not seem to be in the direction of tension, but more random in placement, displayed in both the internal edge (Figure 3.14(A) and 3.15(A)) and internal interior plane sections (Figure 3.14(B) and 3.15(B)). Internal construct interior plane (Figure 3.16(B) and 3.17(B)) had less 
cellular attachment than the constructs of other stimulation protocols (Figure 3.14(B) and 3.15(B)). Both constructs' perpendicular sections (Figure 3.14(C) and 3.15(C)) had numerous visible cells, exhibiting a round morphology, and a majority of the area showed most of the pores completely filled in with extracellular matrix particularly in construct 199A. Additional tension only stimulated construct SEM images are in Appendix A.
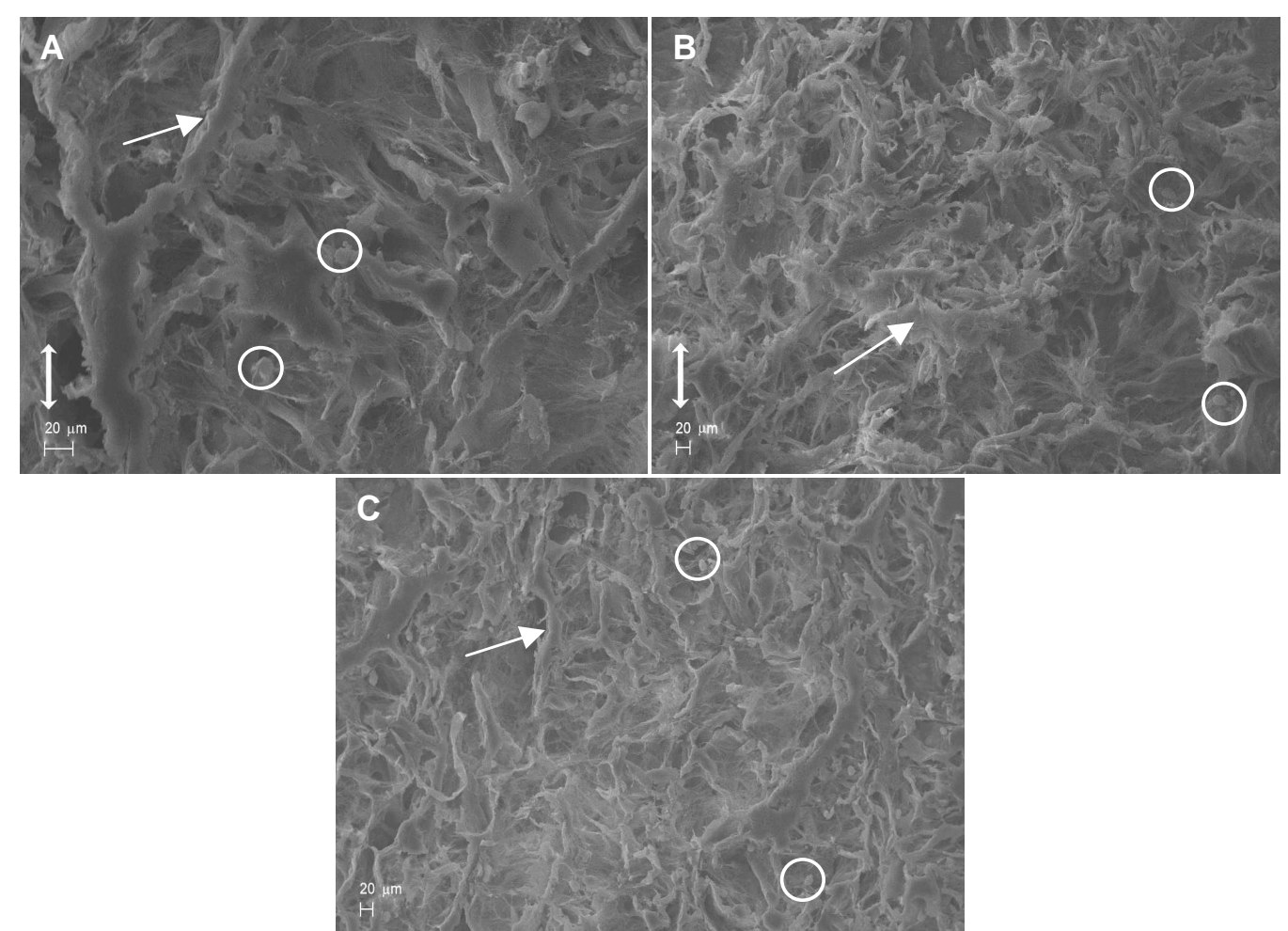

Figure 3.14 Construct 198B with tension only, illustrating an (A) internal edge section, (B) interior plane section parallel to tension, and (C) internal edge section perpendicular to tension (tension direction is going in to picture). White circles indicate cells, white single-headed arrows indicate matrix, and white double-headed arrows indicate direction of tension application (scale bar is $20 \mu \mathrm{m}$ ). 

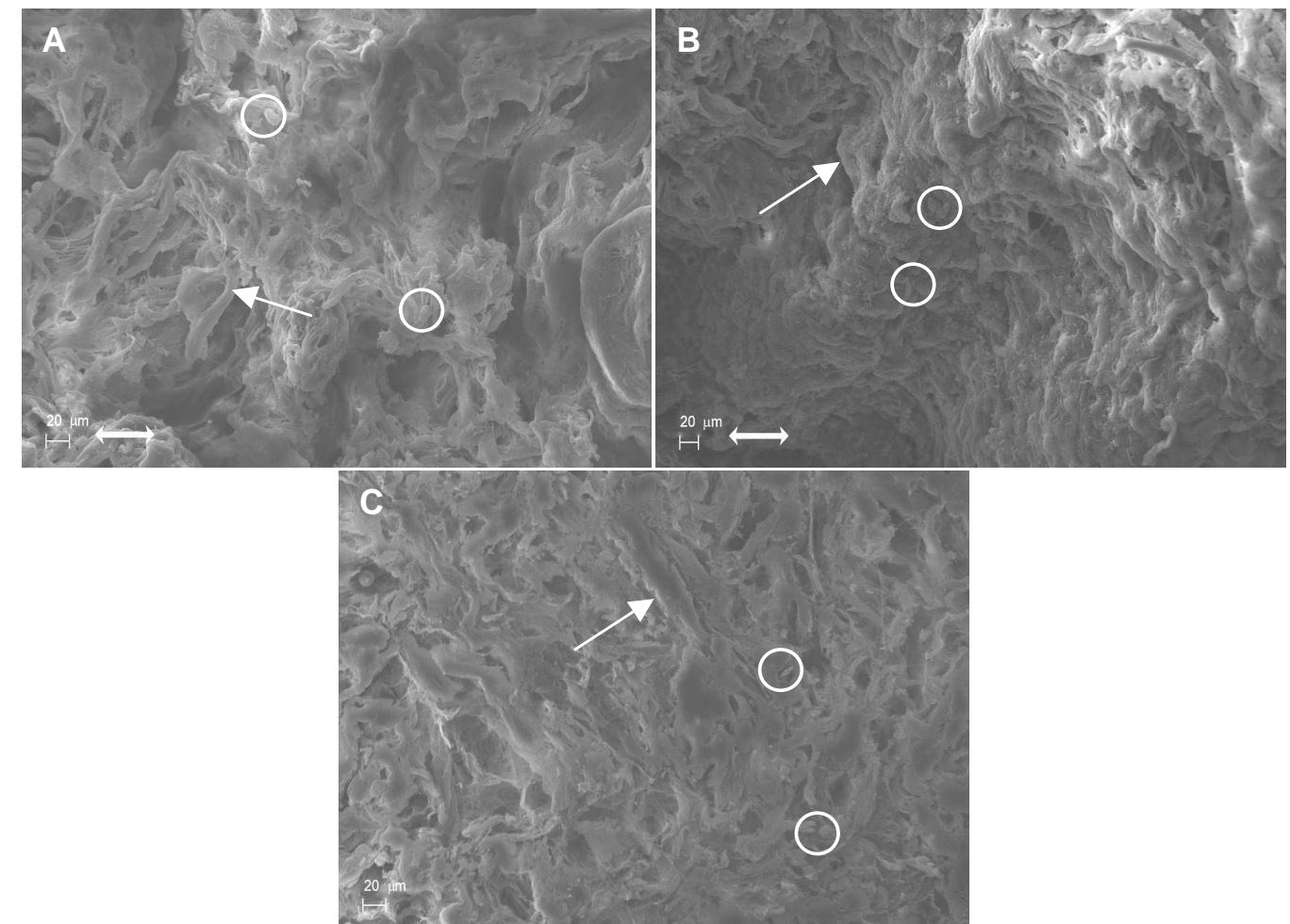

Figure 3.15 Construct 199A with tension only, illustrating an (A) internal edge section, (B) internal parallel to interior plane section, and (C) internal edge section perpendicular to tension (tension direction is going in to picture). White circles indicate cells, white single-headed arrows indicate matrix, and white double-headed arrows indicate direction of tension application (scale bar is $20 \mu \mathrm{m}$ ).

\subsection{CELL ALIGNMENT}

Analysis of preferred cell direction in the constructs involved using the histology images to determine the direction of cellular alignment. Figure 3.16 is a representative image depicting how the cells were circled and processed to determine the angle with respect to the direction of applied tension. The combined results of the angle of alignment analysis are graphed below in a frequency histogram (Figure 3.17). 


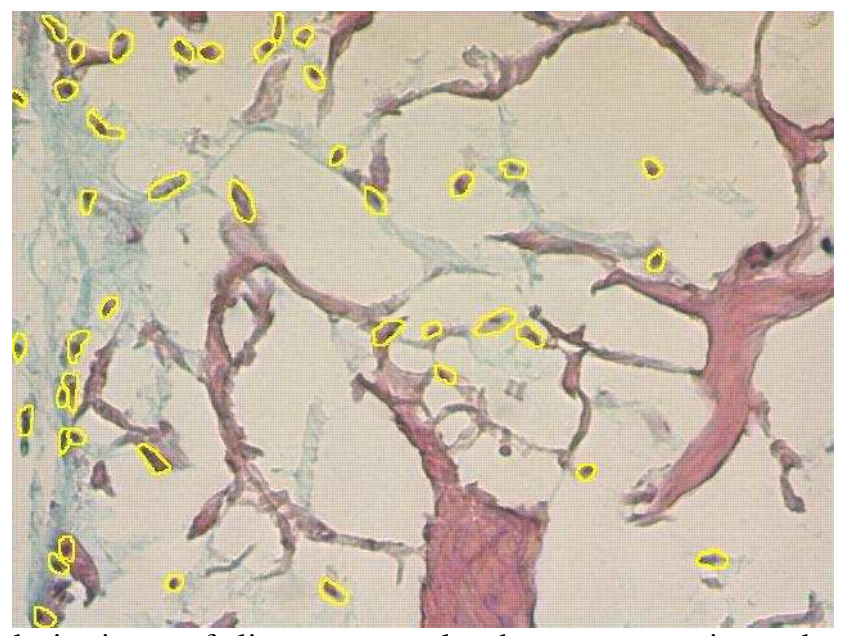

Figure 3.16 Example analysis picture of alignment completed on a compression and tension image of specimen $163 \mathrm{C}$ subjected to compression and tension with no offset (10X).

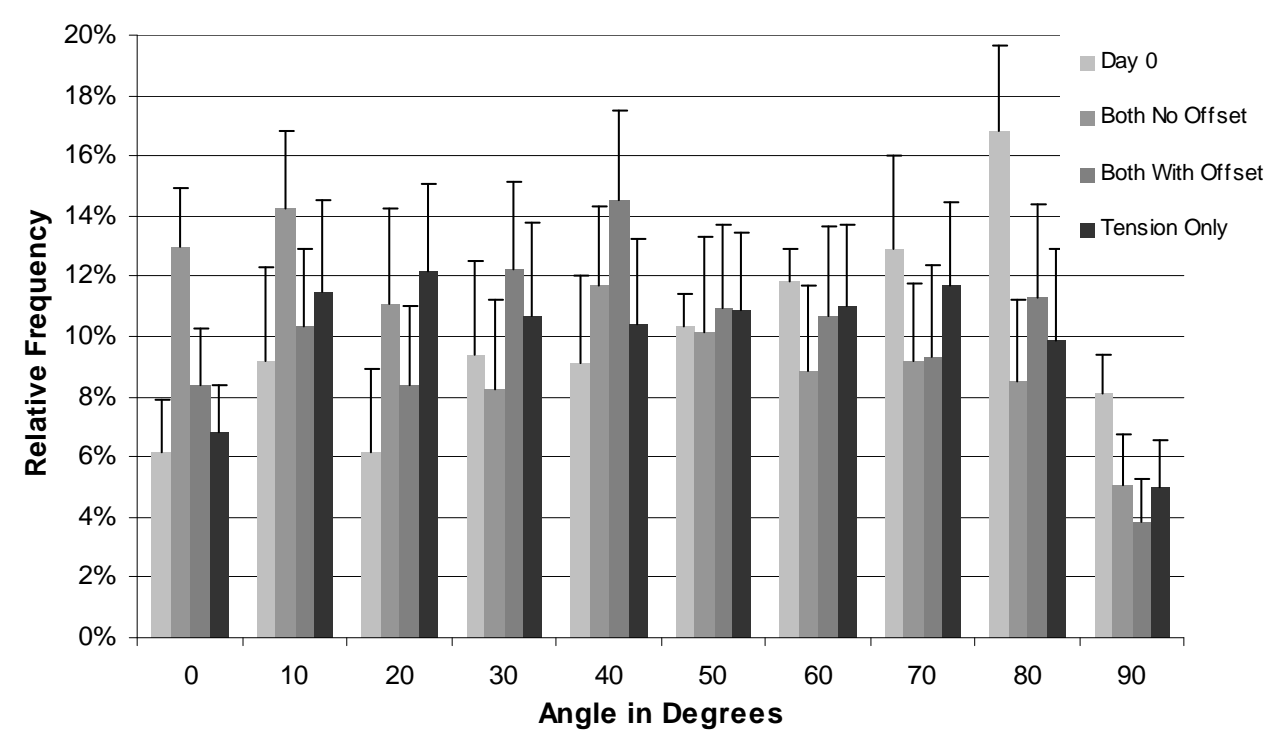

Figure 3.17 Relative frequency for cell alignment in constructs with the respect to the direction of applied tension for all stimulation protocols.

\section{$\underline{\operatorname{Day} O}$}

Day 0 constructs, denoted by white bars (Figure 3.17), showed a greater tendency of cells to be perpendicular to the long axis of the construct and the direction of applied tension. A chart of the Day 0 data only (Figure 3.18) trended toward an 80-degree angle at a relative frequency of greater than $16 \%$ for the constructs' cells. Additionally, the data showed an angle of 50-degrees 
up to 80 -degrees occurred at a relative frequency of $10 \%$ or more. Angles below 50-degrees, except for the 90-degree angle, occurred at a relative frequency found to be below $10 \%$.

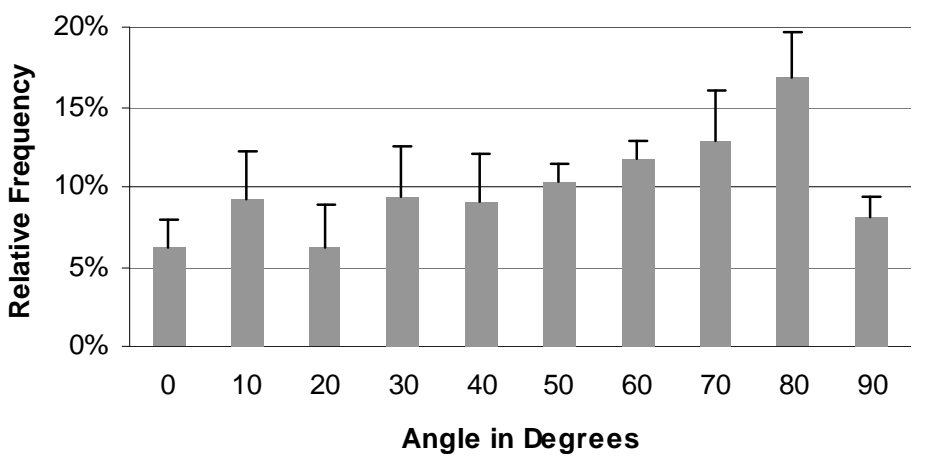

Figure 3.18 A histogram graph representing the alignment data from the Day 0 constructs with no stimulation.

\section{Compression and tension without offset}

Different from the Day 0 data, constructs stimulated with both compression and tension without an offset (denote by light gray bars in Figure 3.17), showed a greater tendency for the cell angle to be aligned with the long axis of the construct, which was the direction of tension application. Constructs stimulated without an offset had cells at angles of 0 to 20 degrees at a relative frequency greater than 10\% (Figure 3.19). Cells at angles greater than 50 degrees occurred less at a relative frequency that was less than $10 \%$.

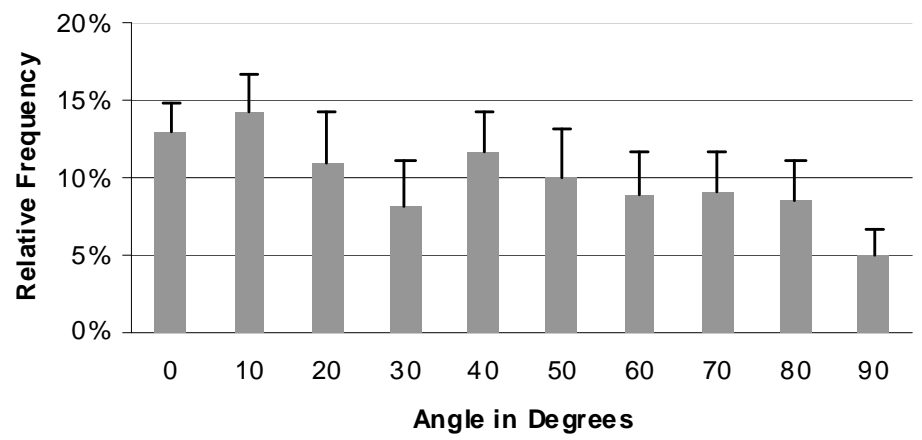

Figure 3.19 A histogram graph representing the alignment data from the stimulated constructs with both compression and tension without offset. 


\section{Compression and tension with offset}

The constructs stimulated with both compression and tension with an offset (denoted by dark gray bars in Figure 3.17) had cells that were more randomly aligned. The relative frequency of angles from 0-degrees up to 80-degrees occurred greater than 8\% (Figure 3.20). Only the 90-degree angle position occurred at a low relative frequency of less than 5\%.

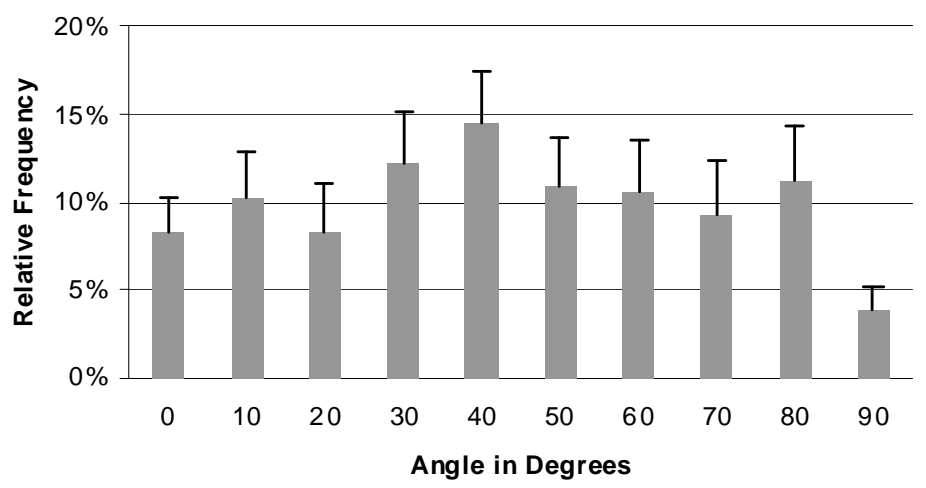

Figure 3.20 A histogram graph representing the alignment data from the stimulated constructs with both compression and tension and an offset.

\section{$\underline{\text { Tension only }}$}

Finally, the tension only stimulated constructs (denoted by black bars in Figure 3.17 ) were similar in appearance to the data for the compression and tension stimulated constructs with an offset in that the cells were more randomly positioned within the construct. Cells at angles 0degrees up to 80-degrees occurred at a relative frequency greater than $8 \%$ and cells at the 90 degree angle occurred at a relative frequency less than 5\% (Figure 3.21). 


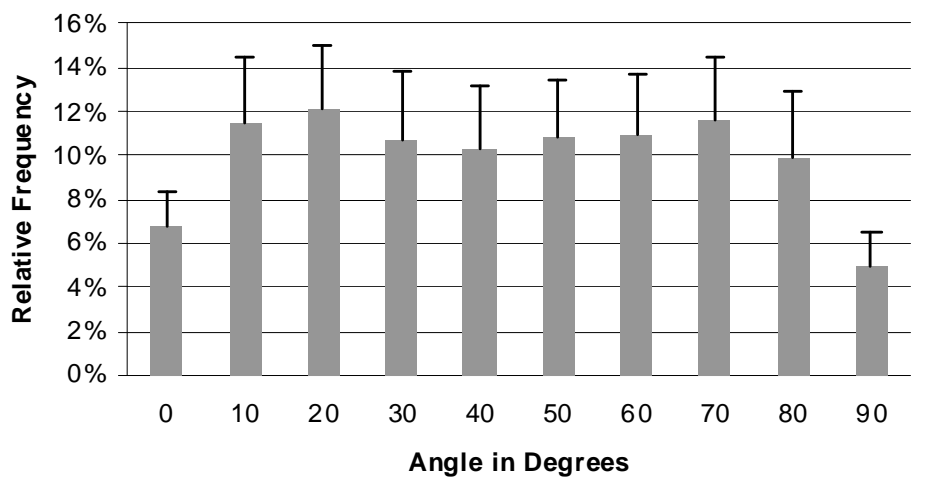

Figure 3.21 A histogram graph representing the alignment data from the stimulated constructs with tension only.

Figure 3.22 below shows the combined average of the cell angle for the constructs of each treatment. Statistical analysis of the alignment data revealed with a one-way ANOVA that there was a statistical difference between the means $(\mathrm{p}<0.00000)$. Post hoc evaluation with a Tukey-Kramer test revealed Day 0 and both compression and tension without offset were significantly different from all groups and each other. Compression and tension with offset and tension only were not statistically different from each other. Day 0 had the majority of its cells at a 50-degree angle, compression and tension with an offset and tension only had cells angled near 44-degrees, and compression and tension without offset had its cells angled near 38-degrees illustrating a trend of compression and tension without offset constructs cells aligning with application of tension. 


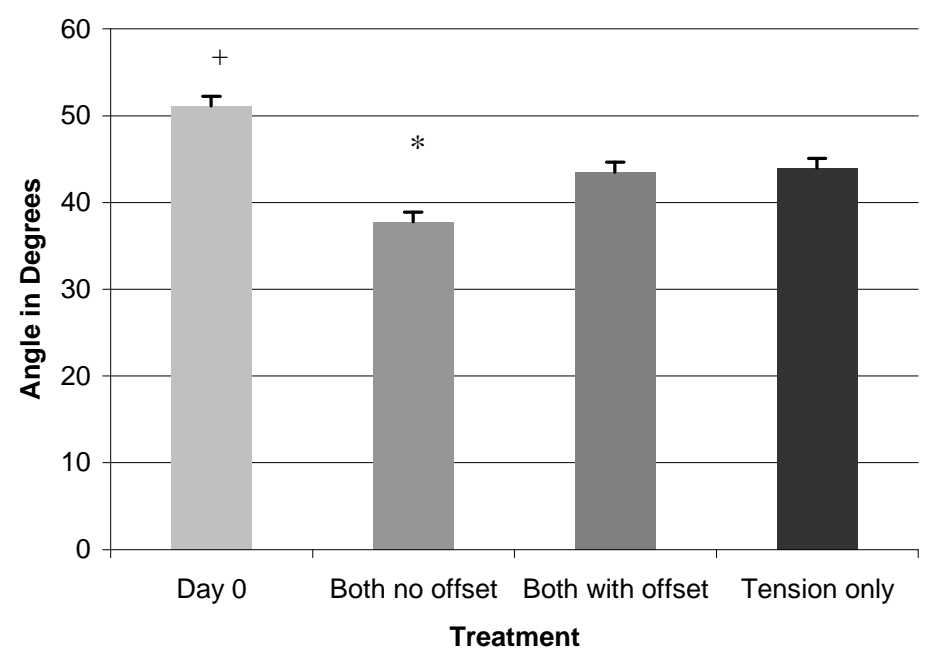

Figure 3.22 Average cell angle for all stimulation protocols in the alignment analysis. (+indicates $p<0.001$ vs. Both no offset, Both with offset, and Tension only;*indicates $\mathrm{p}<0.001$ vs. Both with offset and Tension only.)

\subsection{INDENTATION}

\section{$\underline{\operatorname{Day} 0}$}

Constructs 156A, 156B, 157C, 157D, 158E, 158F, 207A, 207B, and 207C were statically cultured for 1 week prior to indentation testing. The indentation curve-fit graphs are shown in Figures $3.23,3.24$, and 3.25 for these constructs. The measured deformation of each construct began at a small value, except for 157B that had an initial deformation greater than the other constructs in the Day 0 group. As $\mathrm{U} / \mathrm{H}$ increased the predicted curve underestimated the value early on, but as time progressed the predicted curve crossed over the measured and overestimated the curve to the end of the test. The measured curve cross occurred near 100 logarithm time for constructs $156 \mathrm{~A}, 156 \mathrm{~B}$, and $157 \mathrm{C}$ and earlier for the remaining constructs, indicating that the these constructs deformed and reached the predicted curve faster than their counterparts. 

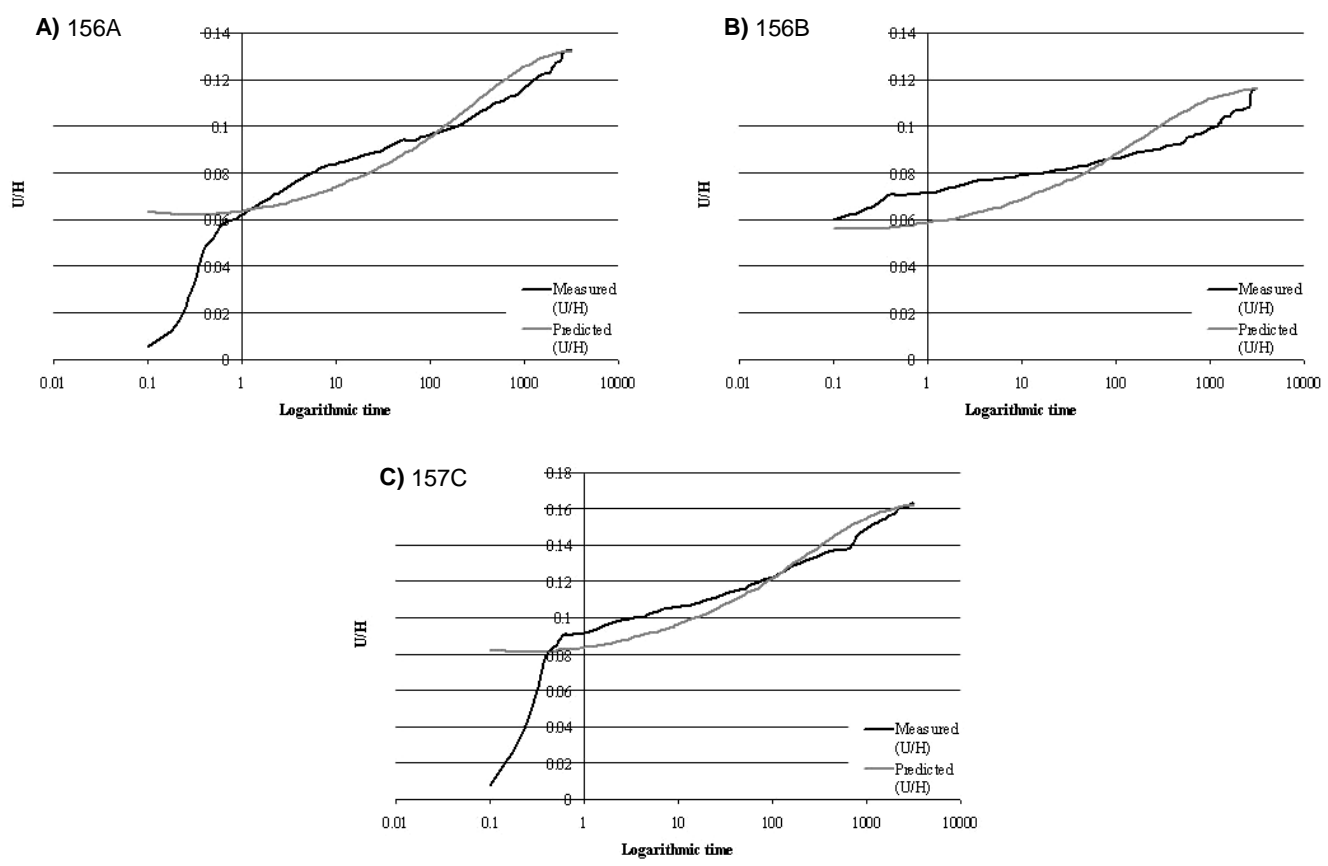

Figure 3.23 Normalized creep response (displacement/construct thickness) from indentation testing of constructs (A) $156 \mathrm{~A}$, (B) 156B, and (C) $157 \mathrm{C}$ and the corresponding curve-fit.
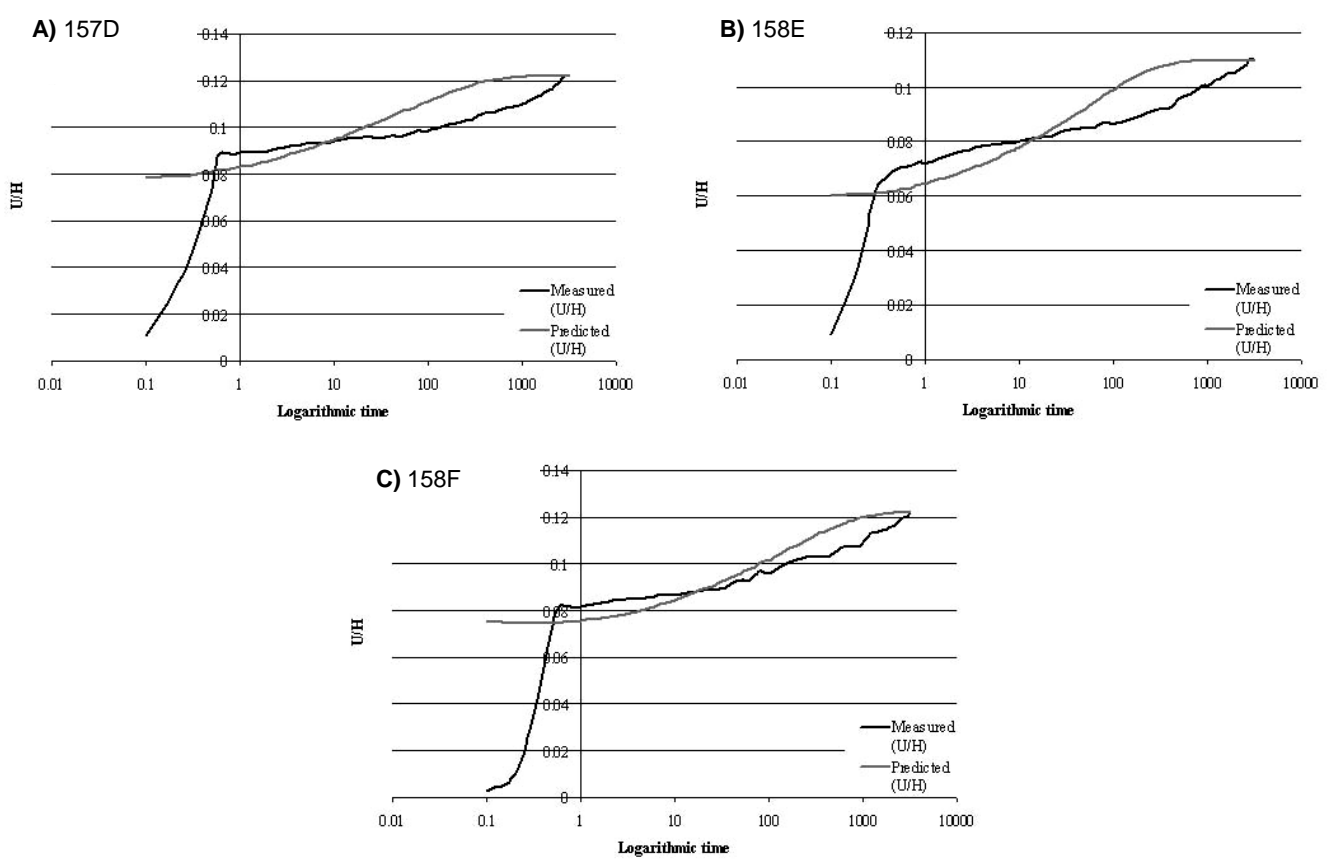

Figure 3.24 Normalized creep response (displacement/construct thickness) from indentation testing of construct (A) $157 \mathrm{D}$, (B) $158 \mathrm{E}$, and (C) $158 \mathrm{~F}$ and the corresponding curve-fit. 

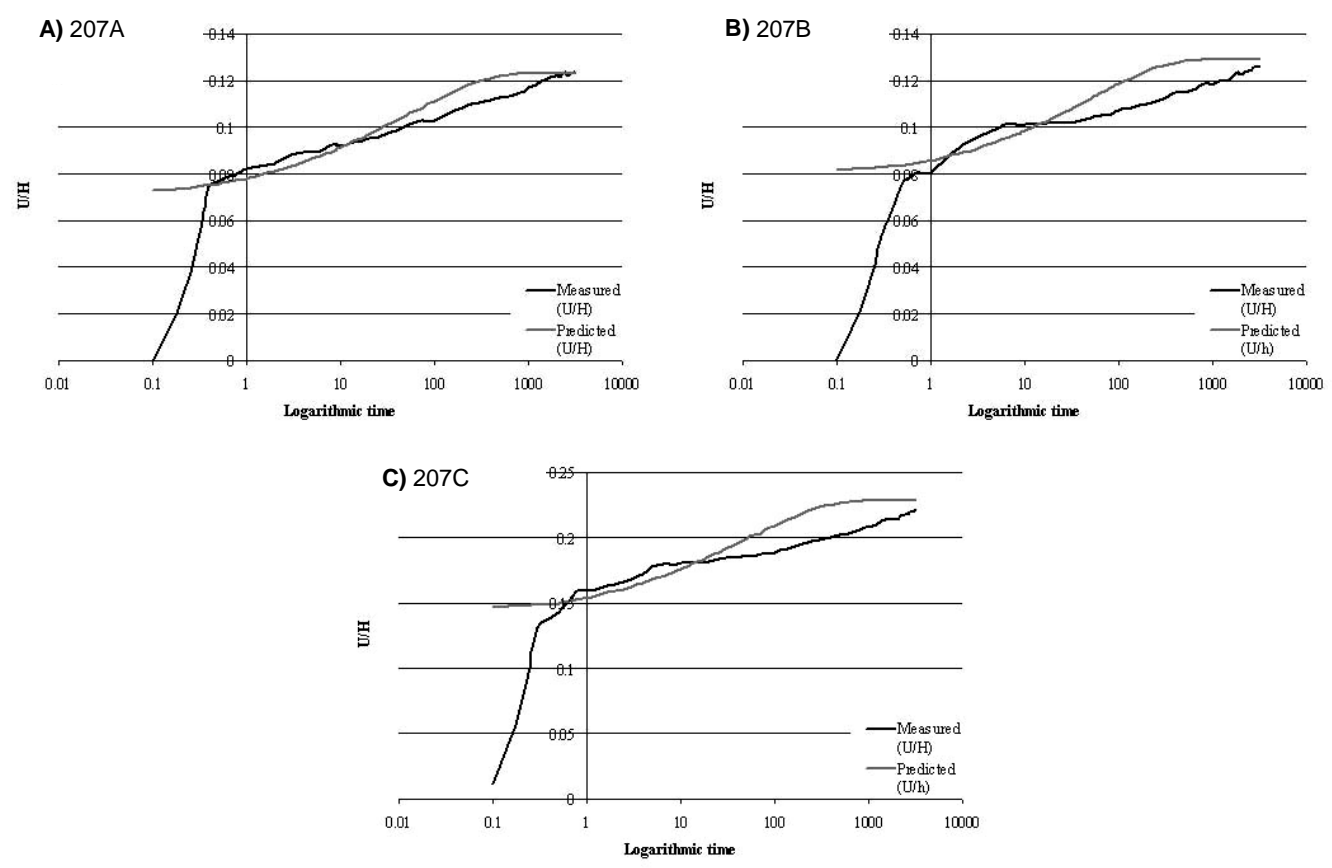

Figure 3.25 Normalized creep response (displacement/construct thickness) from indentation testing of construct (A) 207A, (B) 207B, and (C) 207C and the corresponding curve-fit.

\section{Compression and tension without offset}

Constructs 163A, 163B, 163C, 167B, 167C, 169C, 209A, 209B, and 209C were each stimulated with compression without offset, followed by a rest period, then tension without offset, followed by a rest period and repeated twice daily. The curve-fit graphs for these constructs are in Figures 3.26, 3.27, and 3.28. For all constructs of this group, the initial deformation curve quickly rose above where the predicted curve had expected. For all constructs except 209A and 209C, which were steeper and more similar to Day 0 group, there was less of an incline on the way to the final deformation indicating that the majority of constructs deformed greater initially but not much further as time progressed. For these constructs, the predicted curve crossed the measured curve at 10 logarithm time for all constructs and overestimated the end curve of each of the constructs indicating similarity in behavior for their deformation. The constructs from this group had less deformation at the end than the Day 0 group. 

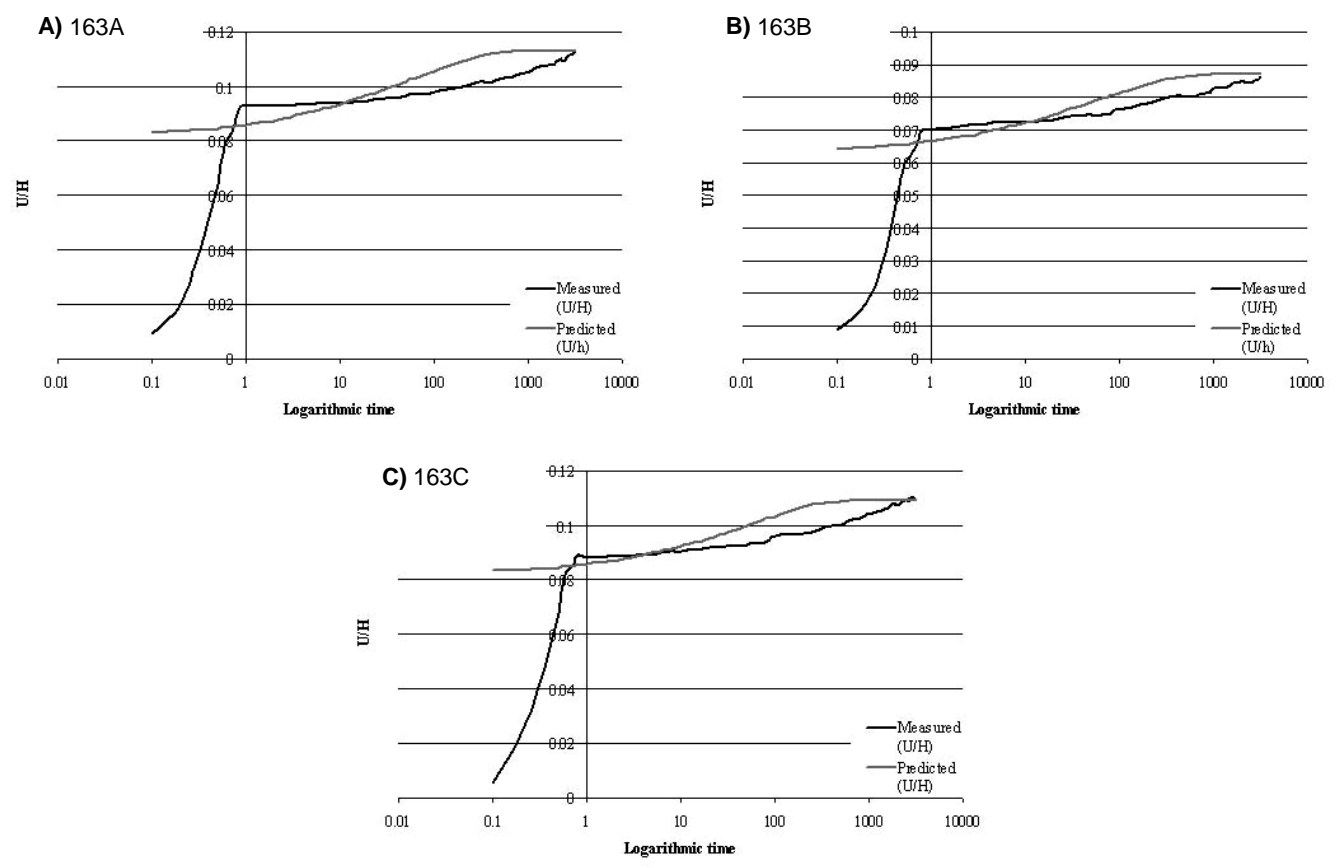

Figure 3.26 Normalized creep response (displacement/construct thickness) from indentation testing of construct (A) $163 \mathrm{~A}$, (B) 163B, and (C) $163 \mathrm{C}$ and the corresponding curve-fit.
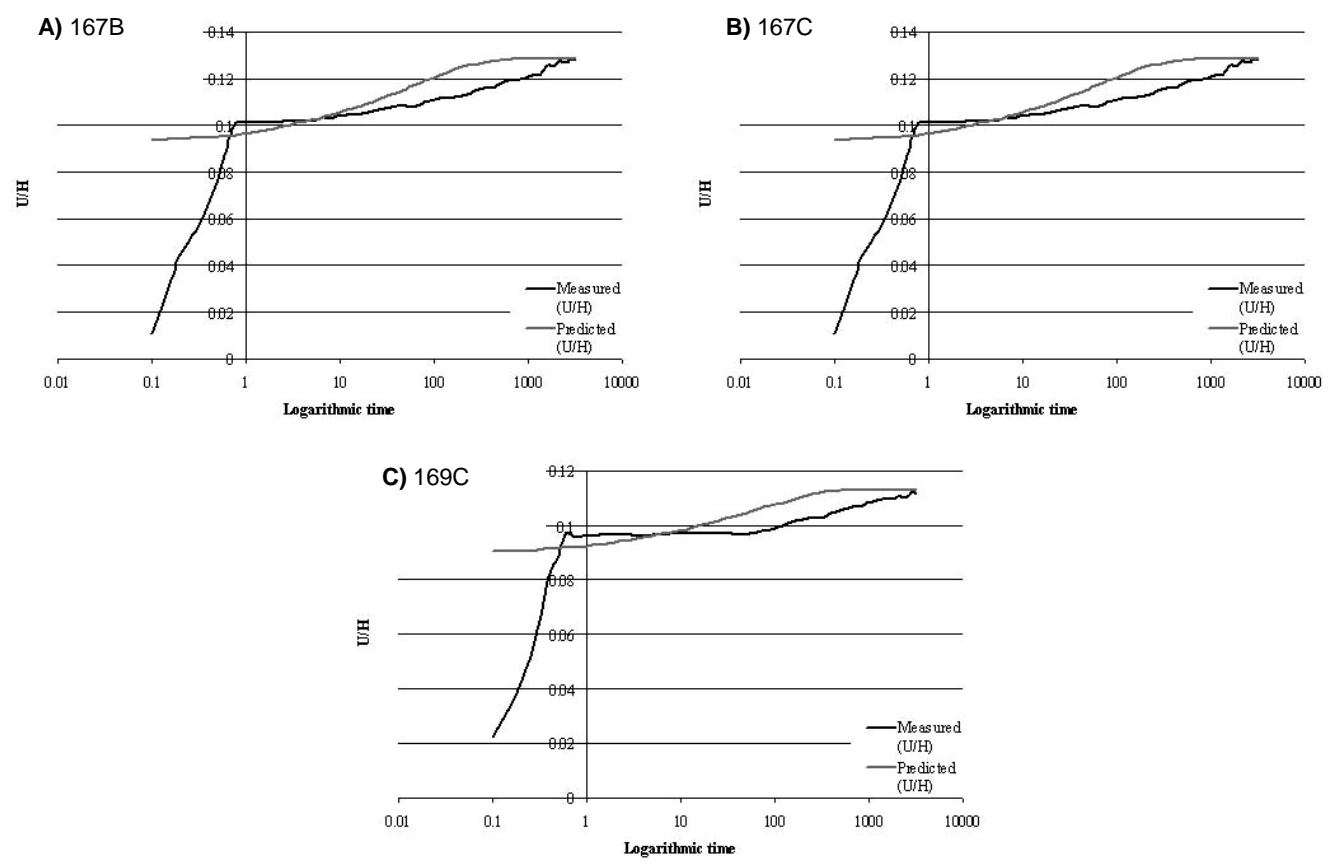

Figure 3.27 Normalized creep response (displacement/construct thickness) from indentation testing of construct (A) $167 \mathrm{~B},(\mathrm{~B}) 167 \mathrm{C}$, and (C) 169C and the corresponding curve-fit. 

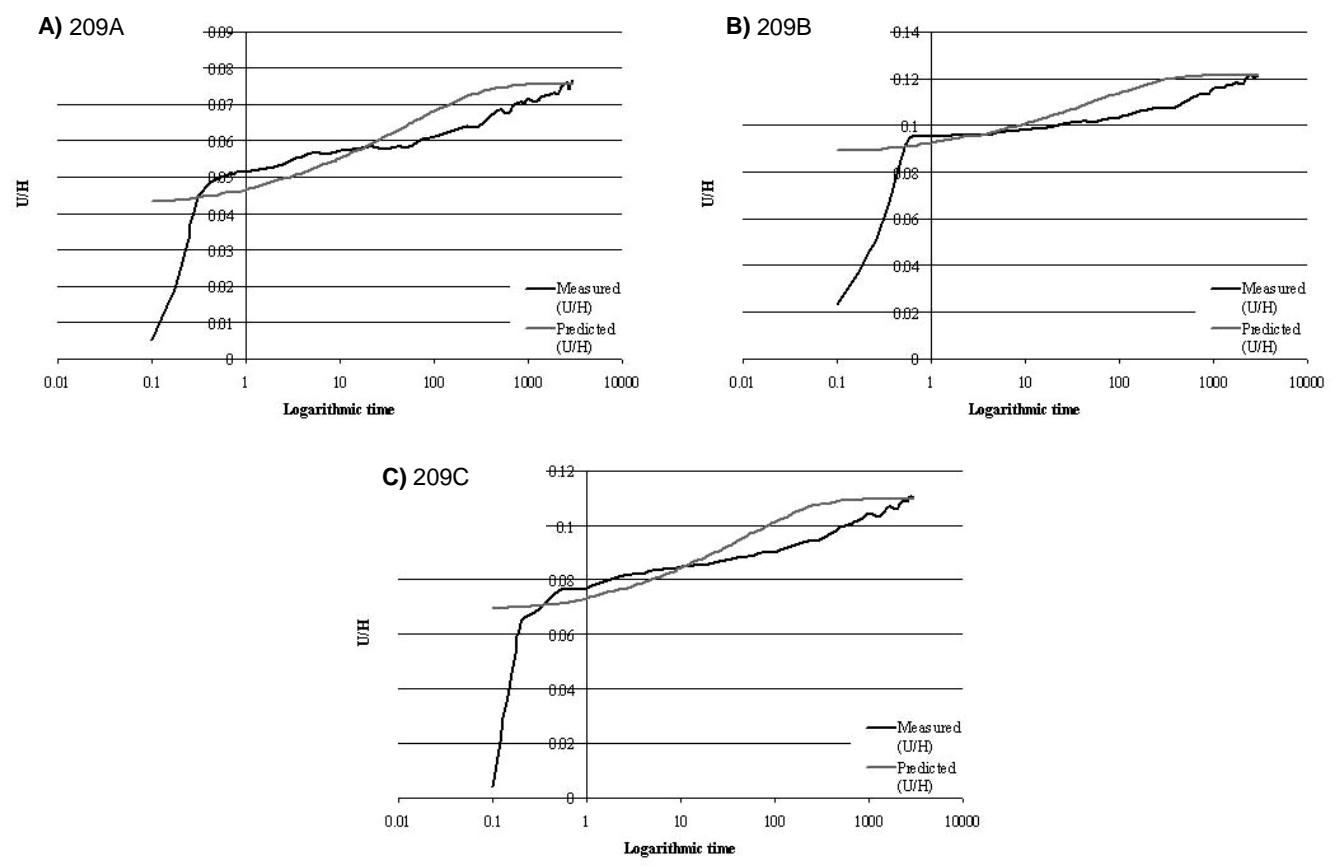

Figure 3.28 Normalized creep response (displacement/construct thickness) from indentation testing of construct (A) 209A, (B) 209B, and (C) 209C and the corresponding curve-fit.

\section{Compression and Tension with offset}

The following constructs 184A, 184B, 184C, 186A, 186C, 188A, 205B, 205C, and 205D were each stimulated with cyclic compression with a static offset, followed by a rest period, then cyclic tension with a static offset, followed by a rest period, which was repeated twice daily for two weeks, then indentation tested. The curve-fit results are shown in Figures 3.29, 3.30, and 3.31 and have a response for all with an initial small $\mathrm{U} / \mathrm{H}$ that immediately increased over a short time, then the rate slowed as the deformation continued. The behavior overall showed a larger $\mathrm{U} / \mathrm{H}$ than most of the both stimulation without offset constructs and a similar curve to that of the Day 0 constructs. The initial response of the measured $\mathrm{U} / \mathrm{H}$ was higher than the predicted estimation, then crossed the measured around 10 logarithmic time, except for construct 184A that crossed closer to 80 logarithmic time, which indicated that most of the constructs in this 
group deformed similarly as they progressed to the final $\mathrm{U} / \mathrm{H}$ value. The predicted curve over estimated the deformation from the intersected time to end of the curve.
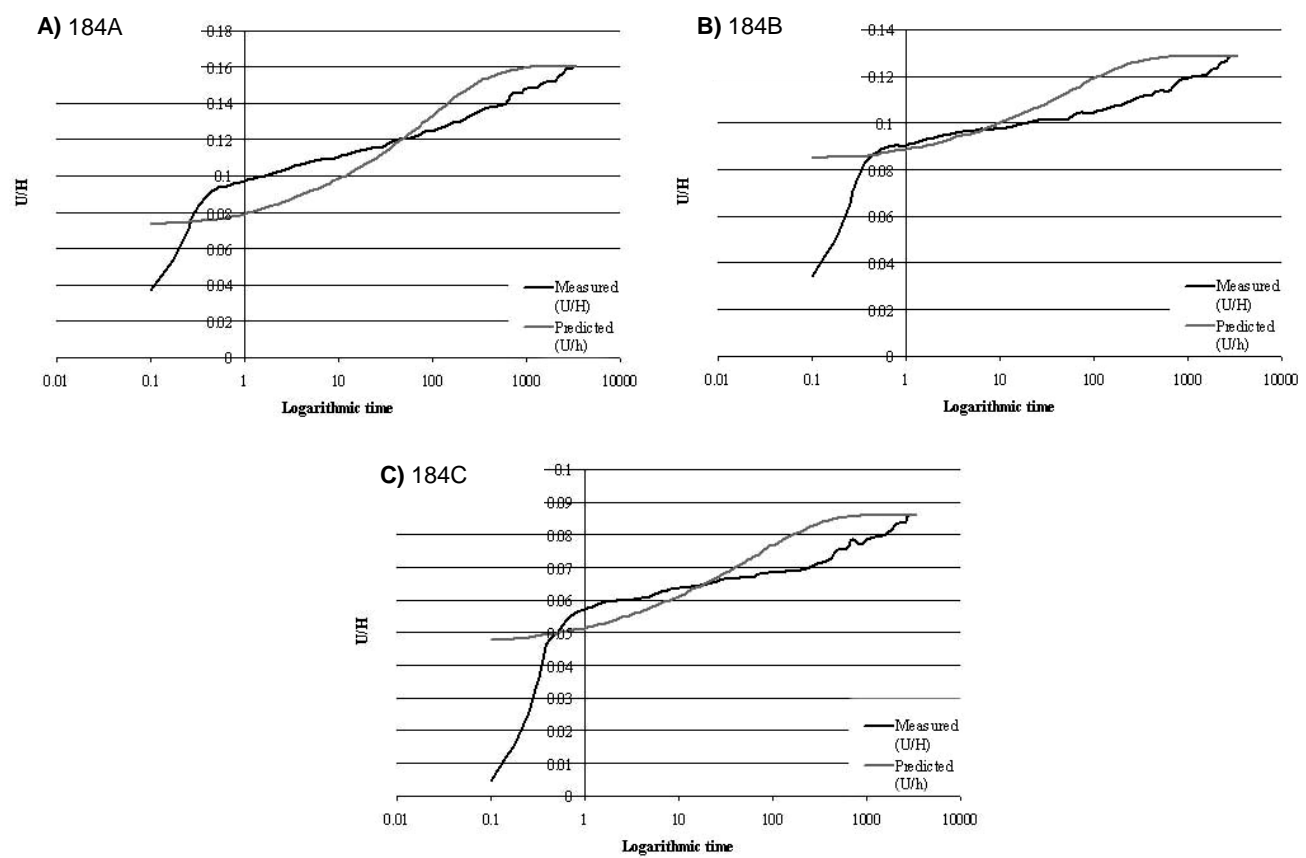

Figure 3.29 Normalized creep response (displacement/construct thickness) from indentation testing of construct (A) $184 \mathrm{~A},(\mathrm{~B}) 184 \mathrm{~B}$, and (C) $184 \mathrm{C}$ and the corresponding curve-fit. 

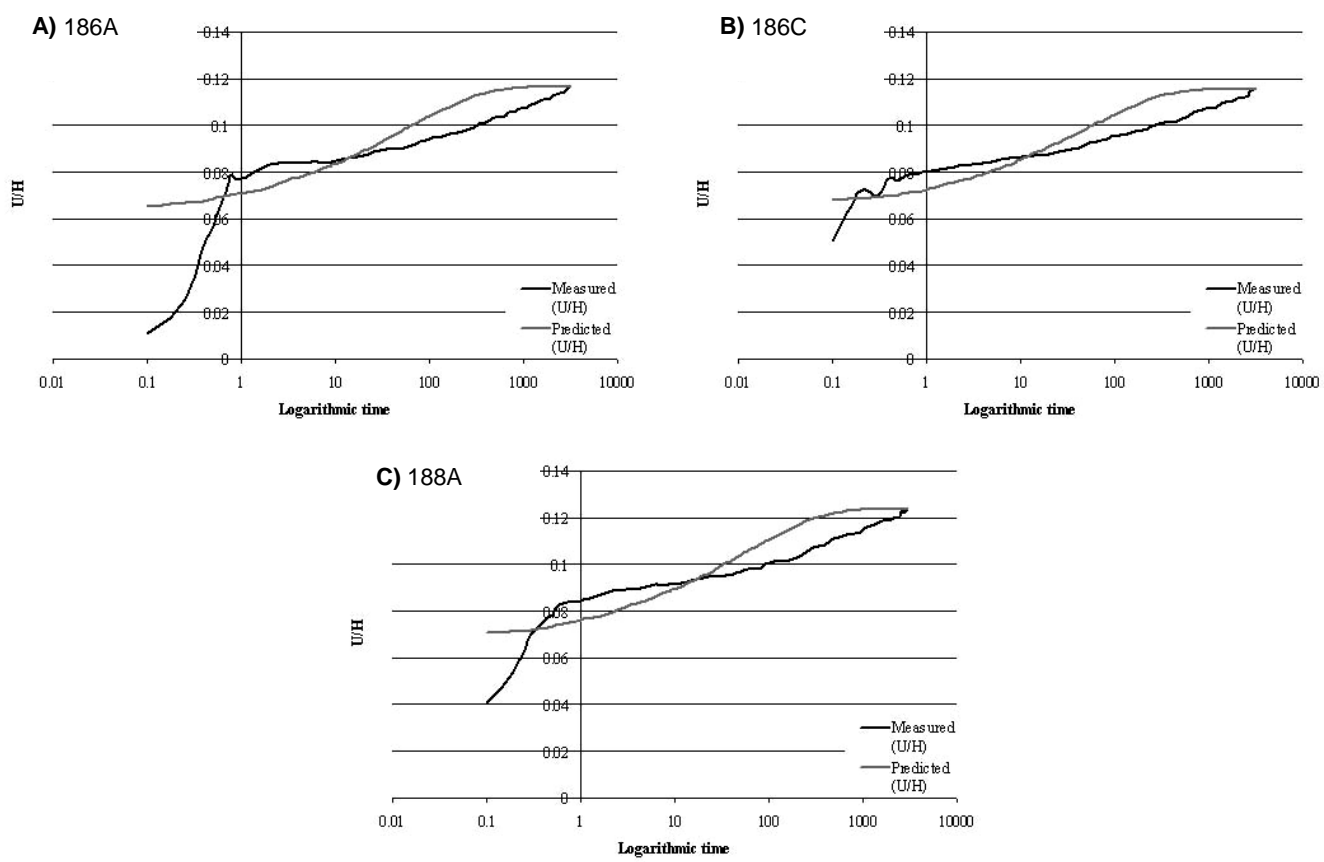

Figure 3.30 Normalized creep response (displacement/construct thickness) from indentation testing of construct (A) $186 \mathrm{~A}$, (B) 186C, and (C) 188A and the corresponding curve-fit.
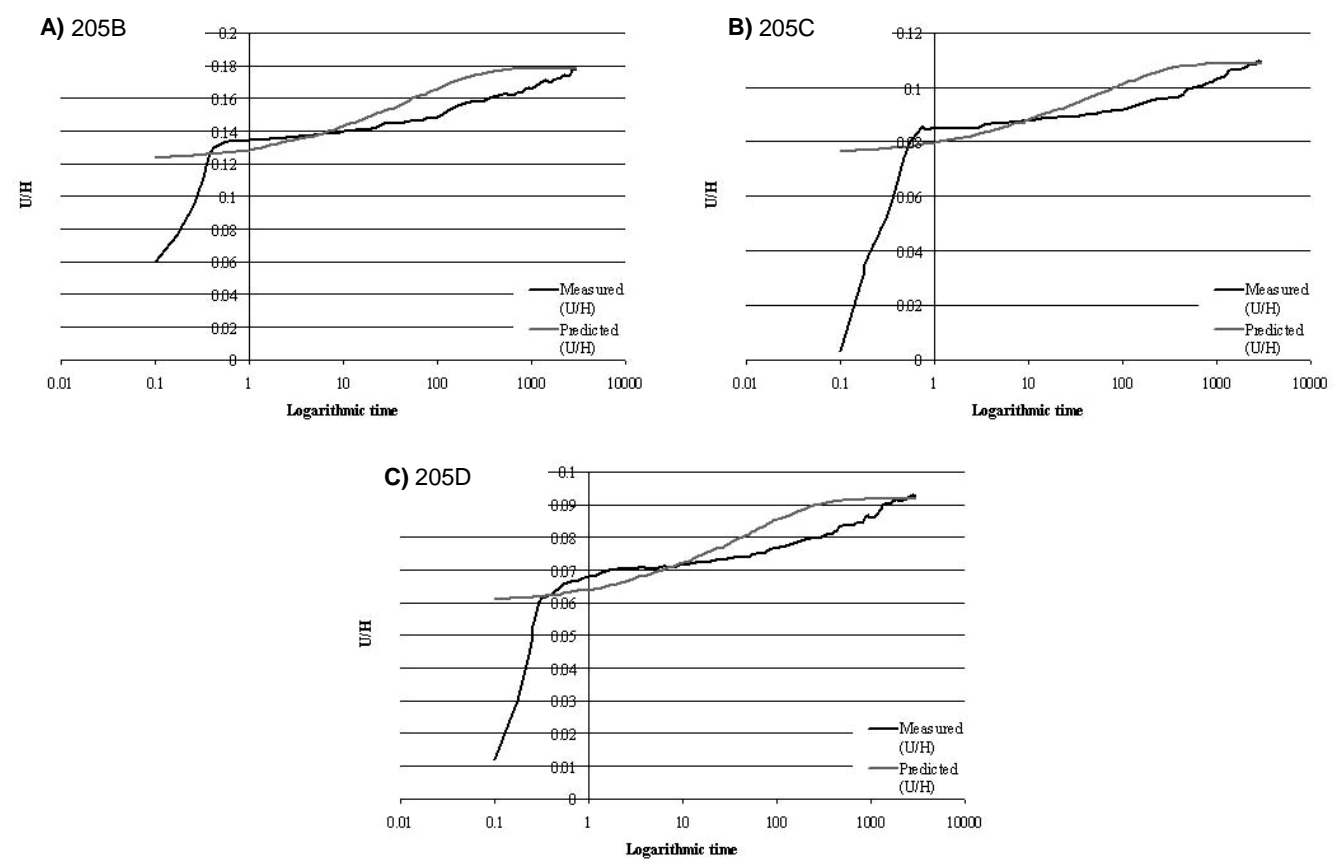

Figure 3.31 Normalized creep response (displacement/construct thickness) from indentation testing of construct (A) 205B, (B) 205C, and (C) 205D and the corresponding curve-fit. 


\section{$\underline{\text { Tension only }}$}

Constructs 178A, 178C, 181A, 197A, 197B, 198B, 208A, 208B, and 208C were each stimulated with cyclic tension three times daily for two weeks and indentation tested. Curve fit graphs for these constructs are in Figures 3.32, 3.33, and 3.34. The measured response for all constructs started low and quickly rose and leveled off within a few seconds of load application. Similar to the Day 0 and both stimulation with offset constructs, the slope progressed steeper up to the final deformation, but the final $\mathrm{U} / \mathrm{H}$ values were more similar to that of the both stimulation without offset constructs. Additionally, the predicted curve underestimated the behavior at the beginning of the curve and overestimated at the end of the curve, which was seen in all the tested constructs.
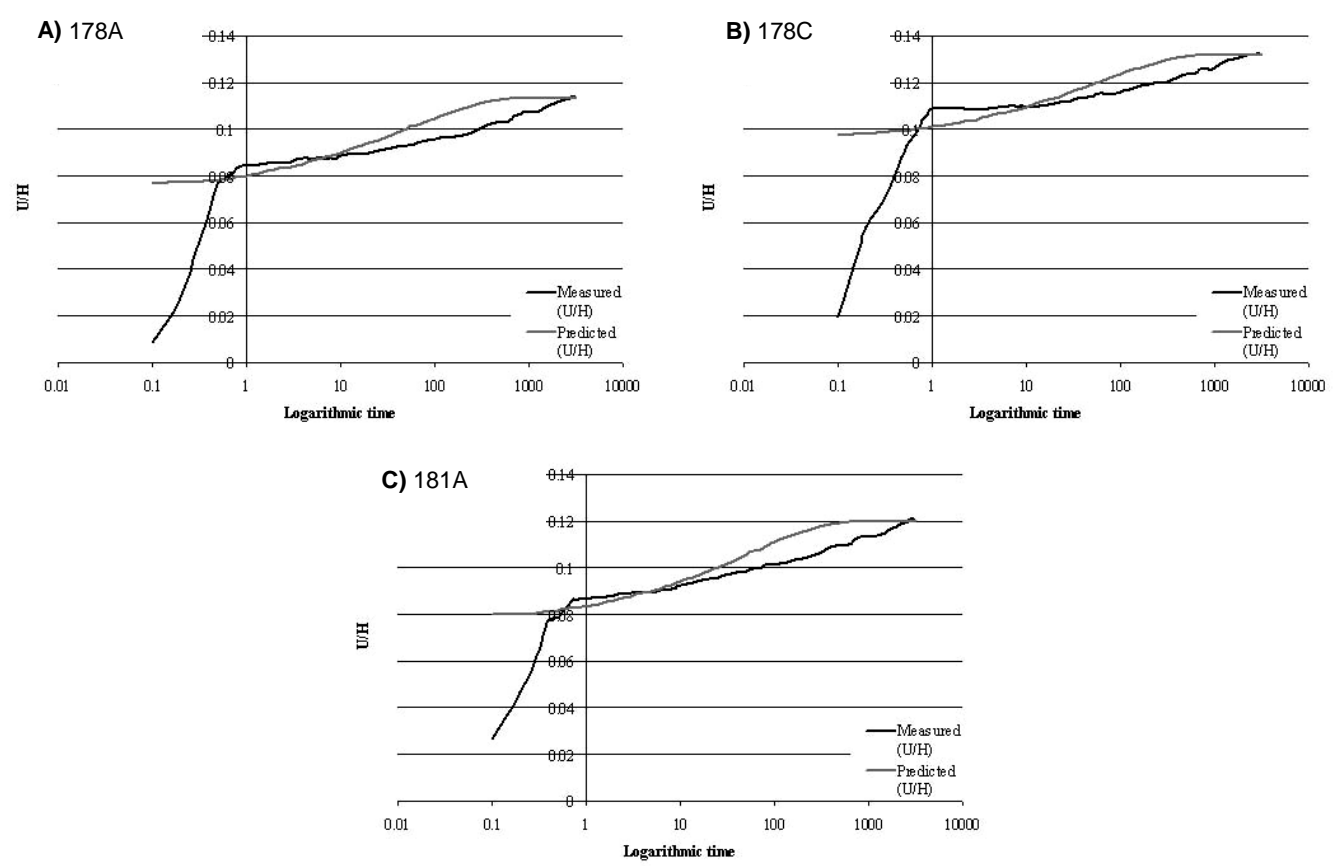

Figure 3.32 Normalized creep response (displacement/construct thickness) from indentation testing of construct (A) $178 \mathrm{~A},(\mathrm{~B}) 178 \mathrm{C}$, and (C) $181 \mathrm{~A}$ and the corresponding curve-fit. 

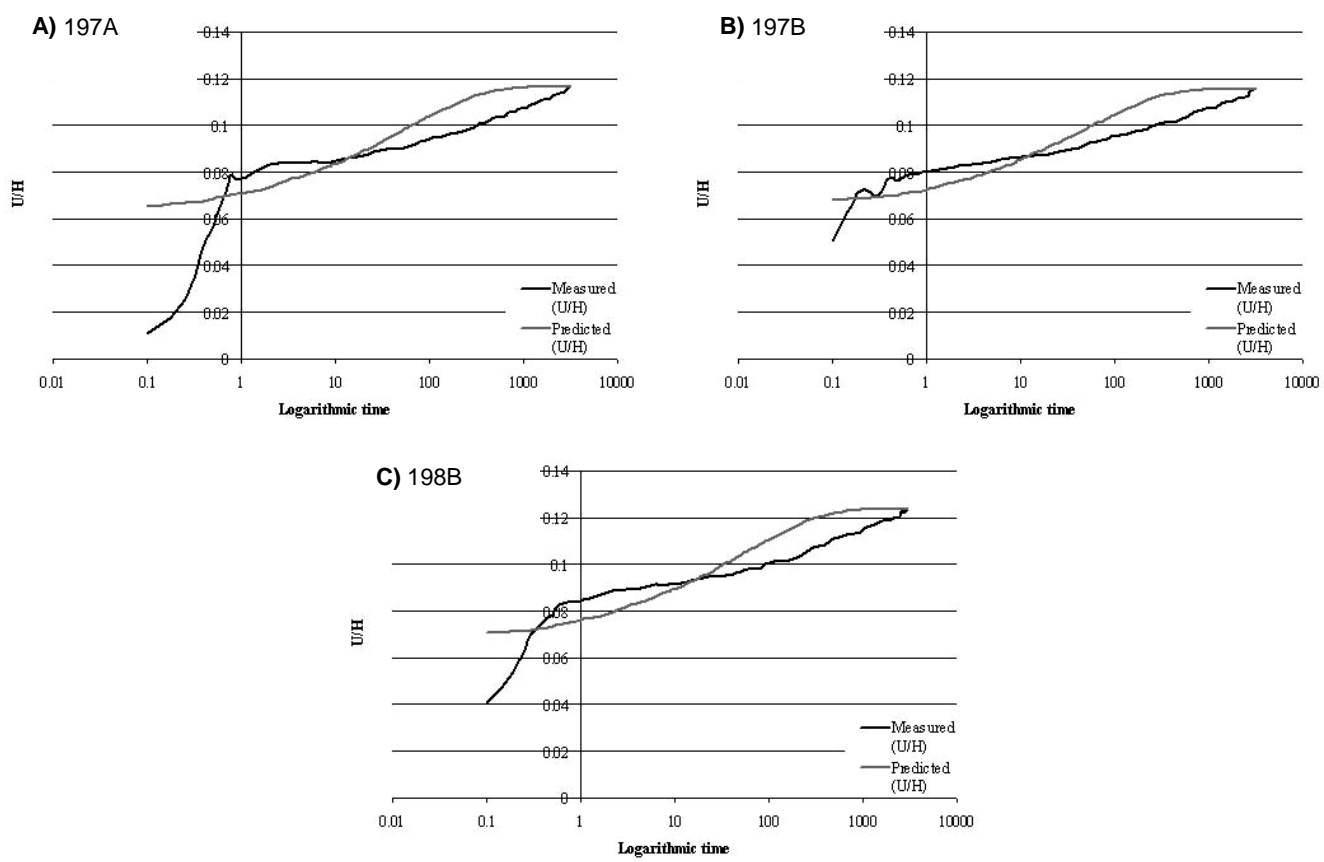

Figure 3.33 Normalized creep response (displacement/construct thickness) from indentation testing of construct (A) 197A, (B) 197B, and (C) 198B and the corresponding curve-fit.
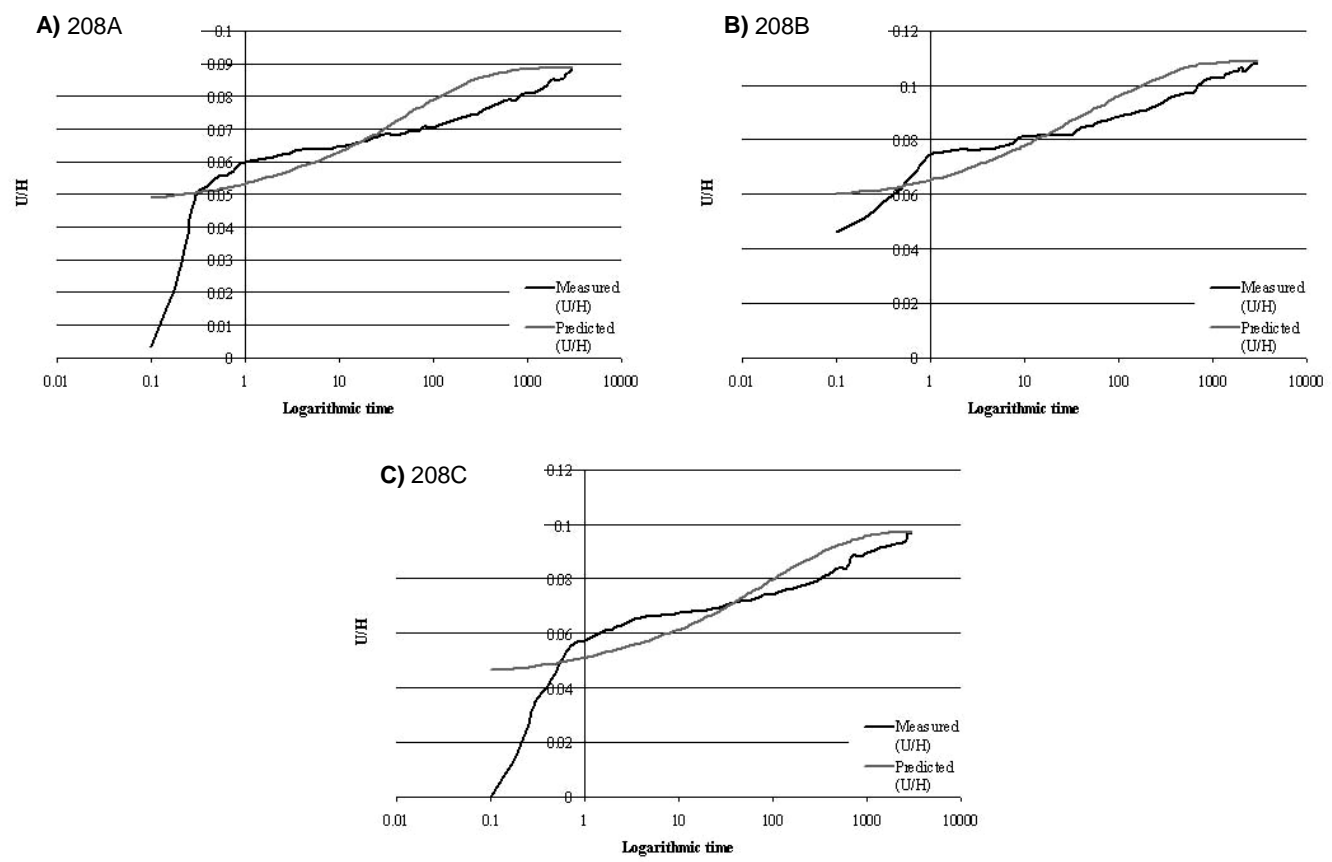

Figure 3.34 Normalized creep response (displacement/construct thickness) from indentation testing of construct (A) 208A, (B) 208B, and (C) 208C and the corresponding curve-fit. 
All curve-fits in this study were over predicted in the beginning and under predicted towards the end of the curve. The program is designed to analyze the data for the best fit throughout the curve. Although not evident on the logarithm time scale, there is a delay in the system response, which is overcome in less than a second. Table 3.2 shows a summary of all the constructs with their respective values for aggregate modulus, permeability, Poisson's ratio, and shear modulus. A statistical analysis of the aggregate modulus data (Figure 3.59) with a oneway ANOVA revealed a significant difference between at least one of the groups $(\mathrm{p}<0.000115)$. Further comparison revealed there was a significant different between stimulation of both compression and tension without offset and all other groups. There was no significant difference between the remaining groups. 


\begin{tabular}{|c|c|c|c|c|c|c|}
\hline Specimen & Stimulation & $\begin{array}{c}\text { Thickness } \\
(\mathrm{mm})\end{array}$ & $\begin{array}{c}\text { Aggregate } \\
\text { Modulus (MPa) }\end{array}$ & $\begin{array}{l}\text { Permeability } \\
\left(m^{\wedge} 4 / N^{\star} s\right)\end{array}$ & $\mathrm{Nu}$ & $\begin{array}{c}\text { Shear Modulus } \\
\text { (MPa) }\end{array}$ \\
\hline $156 \mathrm{~A}$ & Day 0 & 1.98 & 0.018 & $8.29 \mathrm{E}-14$ & 0.00 & 0.009 \\
\hline 156B & Day 0 & 2.22 & 0.019 & $1.18 \mathrm{E}-13$ & 0.00 & 0.010 \\
\hline $157 C$ & Day 0 & 2.10 & 0.014 & $1.27 \mathrm{E}-13$ & 0.05 & 0.007 \\
\hline 157D & Day 0 & 2.40 & 0.019 & $3.67 \mathrm{E}-13$ & 0.26 & 0.006 \\
\hline $158 \mathrm{E}$ & Day 0 & 1.70 & 0.023 & $3.05 E-13$ & 0.16 & 0.009 \\
\hline $158 \mathrm{~F}$ & Day 0 & 2.00 & 0.021 & $1.14 \mathrm{E}-13$ & 0.24 & 0.007 \\
\hline $207 \mathrm{~A}$ & Day 0 & 2.17 & 0.019 & $3.64 \mathrm{E}-13$ & 0.21 & 0.007 \\
\hline 207B & Day 0 & 1.87 & 0.021 & $3.30 \mathrm{E}-13$ & 0.26 & 0.007 \\
\hline \multirow[t]{3}{*}{$207 C$} & Day 0 & 2.13 & 0.011 & $6.36 \mathrm{E}-13$ & 0.26 & 0.004 \\
\hline & Mean & 2.06 & 0.018 & $2.715 \mathrm{E}-13$ & 0.16 & 0.007 \\
\hline & Stdev & 0.20 & 0.004 & 1.803E-13 & 0.11 & 0.002 \\
\hline $163 \mathrm{~A}$ & Both without offset & 2.12 & 0.026 & $2.68 \mathrm{E}-13$ & 0.34 & 0.006 \\
\hline $163 B$ & Both without offset & 2.12 & 0.033 & $2.12 E-13$ & 0.34 & 0.008 \\
\hline $163 C$ & Both without offset & 2.21 & 0.031 & $2.27 \mathrm{E}-13$ & 0.37 & 0.007 \\
\hline 167B & Both without offset & 1.94 & 0.024 & $2.89 E-13$ & 0.34 & 0.006 \\
\hline $167 C$ & Both without offset & 1.77 & 0.028 & $2.67 \mathrm{E}-13$ & 0.34 & 0.007 \\
\hline $169 \mathrm{C}$ & Both without offset & 2.04 & 0.033 & $2.12 \mathrm{E}-13$ & 0.39 & 0.006 \\
\hline $209 A$ & Both without offset & 2.13 & 0.031 & $2.39 \mathrm{E}-13$ & 0.18 & 0.012 \\
\hline $209 B$ & Both without offset & 2.11 & 0.024 & 3.07E-13 & 0.34 & 0.006 \\
\hline \multirow[t]{3}{*}{$209 \mathrm{C}$} & Both without offset & 1.92 & 0.025 & $3.04 \mathrm{E}-13$ & 0.26 & 0.008 \\
\hline & Mean & 2.04 & 0.028 & $2.585 \mathrm{E}-13$ & 0.32 & 0.007 \\
\hline & Stdev & 0.14 & 0.004 & 3.733E-14 & 0.06 & 0.002 \\
\hline $184 \mathrm{~A}$ & Both with offset & 1.85 & 0.016 & $2.87 \mathrm{E}-13$ & 0.00 & 0.008 \\
\hline 184B & Both with offset & 1.80 & 0.023 & $2.92 \mathrm{E}-13$ & 0.29 & 0.007 \\
\hline $184 C$ & Both with offset & 2.04 & 0.028 & $2.37 \mathrm{E}-13$ & 0.16 & 0.011 \\
\hline $186 \mathrm{~A}$ & Both with offset & 2.16 & 0.020 & $3.54 \mathrm{E}-13$ & 0.16 & 0.008 \\
\hline $186 \mathrm{C}$ & Both with offset & 2.07 & 0.021 & $3.31 \mathrm{E}-13$ & 0.21 & 0.008 \\
\hline $188 \mathrm{~A}$ & Both with offset & 1.90 & 0.030 & $2.51 \mathrm{E}-13$ & 0.32 & 0.008 \\
\hline 205B & Both with offset & 1.90 & 0.017 & 4.23E-13 & 0.32 & 0.005 \\
\hline $205 C$ & Both with offset & 2.16 & 0.025 & $2.80 \mathrm{E}-13$ & 0.32 & 0.007 \\
\hline \multirow[t]{3}{*}{ 205D } & Both with offset & 1.96 & 0.030 & $2.48 \mathrm{E}-13$ & 0.29 & 0.009 \\
\hline & Mean & 1.98 & 0.023 & $3.002 E-13$ & 0.23 & 0.008 \\
\hline & Stdev & 0.13 & 0.005 & $5.980 \mathrm{E}-14$ & 0.11 & 0.002 \\
\hline $178 \mathrm{~A}$ & Tension only & 2.22 & 0.023 & $3.13 E-13$ & 0.29 & 0.007 \\
\hline $178 \mathrm{C}$ & Tension only & 2.18 & 0.022 & $3.20 \mathrm{E}-13$ & 0.34 & 0.005 \\
\hline $181 \mathrm{~A}$ & Tension only & 1.96 & 0.023 & $3.04 \mathrm{E}-13$ & 0.29 & 0.007 \\
\hline $197 A$ & Tension only & 2.07 & 0.018 & $3.94 \mathrm{E}-13$ & 0.26 & 0.006 \\
\hline 197B & Tension only & 2.19 & 0.025 & $2.96 \mathrm{E}-13$ & 0.13 & 0.011 \\
\hline $198 \mathrm{~B}$ & Tension only & 2.28 & 0.018 & 4.16E-13 & 0.16 & 0.007 \\
\hline $208 \mathrm{~A}$ & Tension only & 2.23 & 0.025 & $2.96 \mathrm{E}-13$ & 0.13 & 0.011 \\
\hline 208B & Tension only & 2.69 & 0.018 & $3.99 \mathrm{E}-13$ & 0.16 & 0.007 \\
\hline \multirow[t]{3}{*}{$208 \mathrm{C}$} & Tension only & 2.46 & 0.021 & $2.11 \mathrm{E}-13$ & 0.00 & 0.011 \\
\hline & Mean & 2.25 & 0.021 & 3.276E-13 & 0.20 & 0.008 \\
\hline & Stdev & 0.21 & 0.003 & $6.472 \mathrm{E}-14$ & 0.11 & 0.002 \\
\hline
\end{tabular}

Table 3.1 Data summary for all constructs indentation tested. 


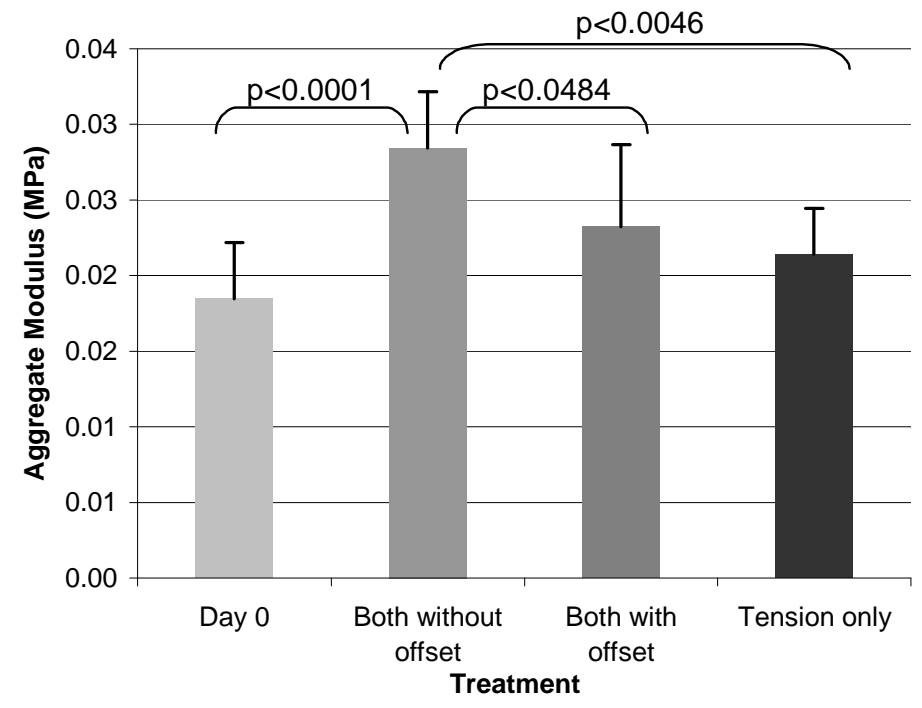

Figure 3.35 Aggregate modulus for all groups.

For permeability (Figure 3.60), no significant difference was found between any of the groups when compared with a one-way ANOVA.

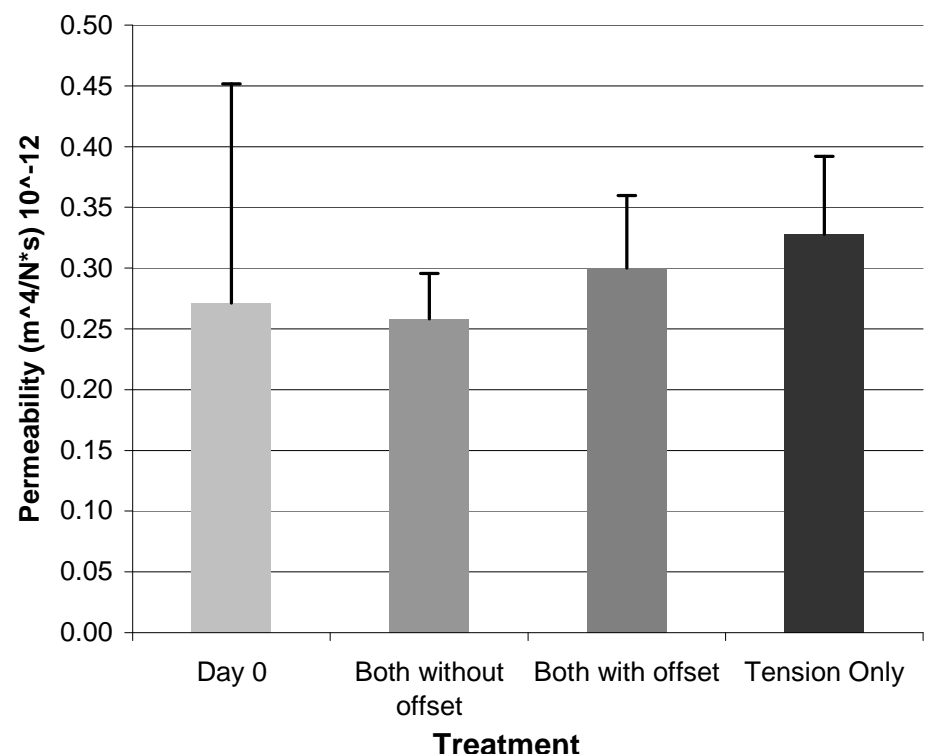

Figure 3.36 Permeability for all groups.

For Poisson's ratio (Figure 3.61), results of a one-way ANOVA showed a significant difference between the means of the groups ( $\mathrm{p}<0.010107)$. Multiple-comparisons showed both 
compression and tension without offset was significantly different from Day 0 and tension only, but not from both compression and tension with offset. Additionally, there were no significant differences between Day 0 and tension only.

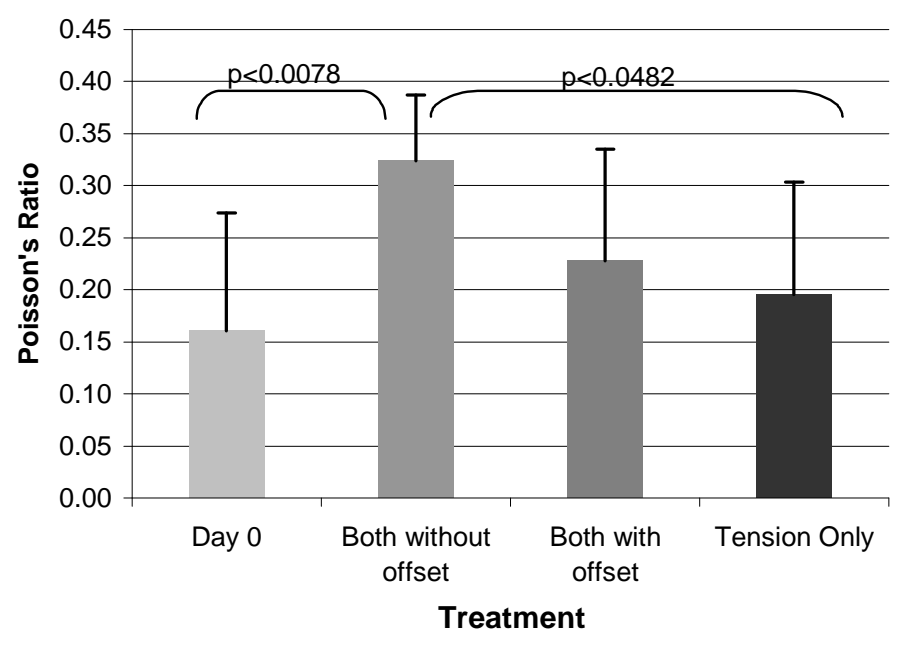

Figure 3.37 Poisson's ratio for all groups.

\subsection{TENSION TESTING}

$\underline{\operatorname{Day} O}$

The following constructs were statically cultured for 7 days prior to mechanically testing with indentation and following recovery, tested by tension testing to failure: 156A, 156B, 157C, 157D, 158E, 158F, 207A, 207B, and 207C.

The stress versus strain curves for constructs 156A, $156 \mathrm{~B}, 157 \mathrm{C}, 157 \mathrm{D}$, and 158E are shown in Figure 3.38. All responses showed a small toe region, after which the stress-strain response was proportional. Some yielding occurred prior to the peak stress for all constructs except $158 \mathrm{E}$, which instantly failed and returned to zero stress. Once the peak was reach, the 

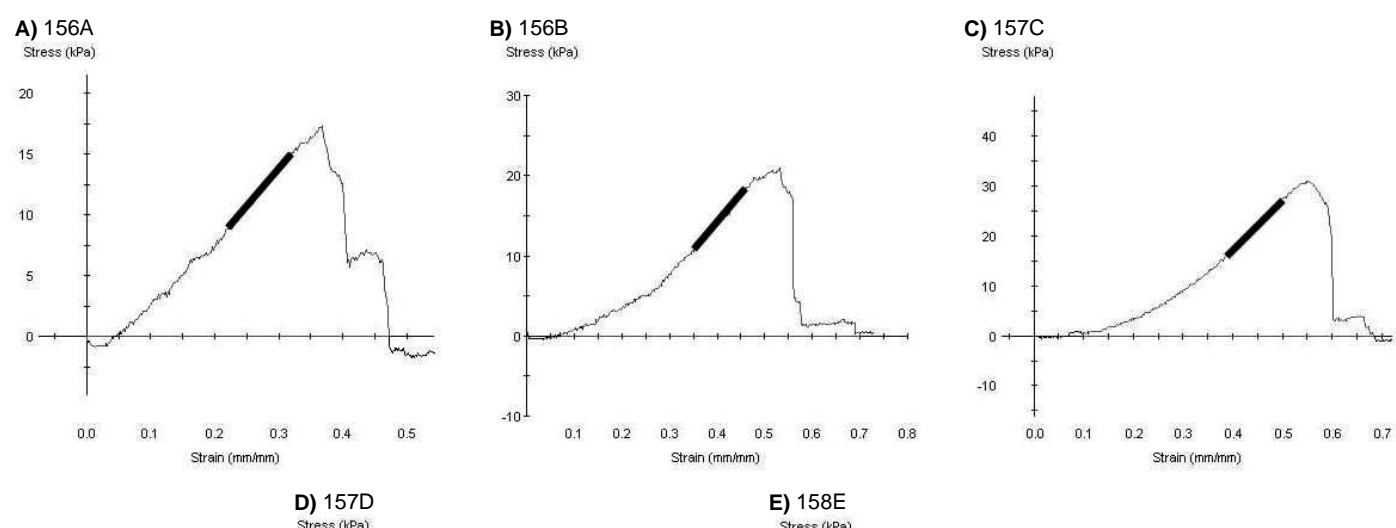

D) $157 \mathrm{D}$
Stress $(\mathrm{kPa})$
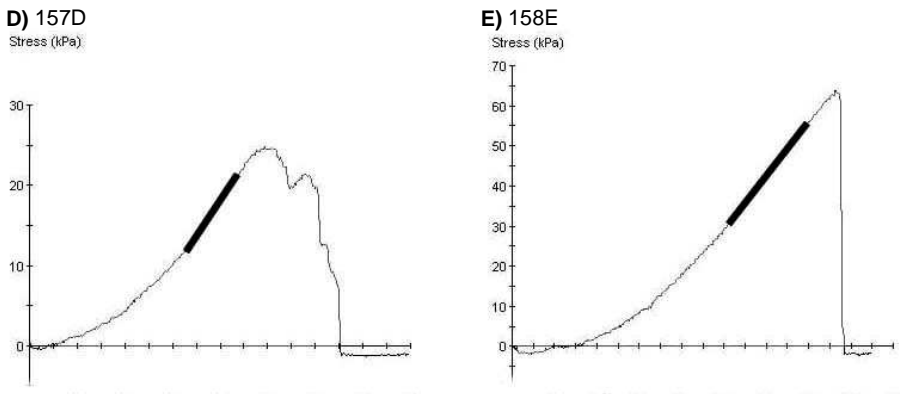

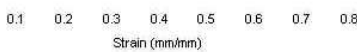

$\begin{array}{llllllllll}0.1 & 0.2 & 0.3 & 0.4 & 0.5 & 0.6 & 0.7 & 0.8 & 0.9\end{array}$

Figure 3.38 Stress versus strain curves for constructs (A) 156A, (B) 156B, (C) 157C, (D) 157D, and (E) 158E; the black line indicates the region used for calculation of modulus.

remaining constructs, continued to break and decreased in stress until each construct was completely severed and a zero stress state was attained. Modulus values for 156A, 156B, 157C, $157 \mathrm{D}$, and 158E were calculated as $60.3,69.1,71.2,85$, and $138 \mathrm{kPa}$, respectively. The stress strain curves (Figure 3.39) for constructs 158F, 207A, 207B, and 207C each exhibited a toe region before the reaction changed over to a linear response. At the peak stress there was rupture, which decreased in stress as the constructs continued to break followed by complete failure with a rapid return to zero stress. The modulus was calculated as $103 \mathrm{kPa}$ for $158 \mathrm{~F}$, as $117 \mathrm{kPa}$ for $207 \mathrm{~A}$, as $85 \mathrm{kPa}$ for $207 \mathrm{~B}$, and as $122.1 \mathrm{kPa}$ for $207 \mathrm{C}$. 

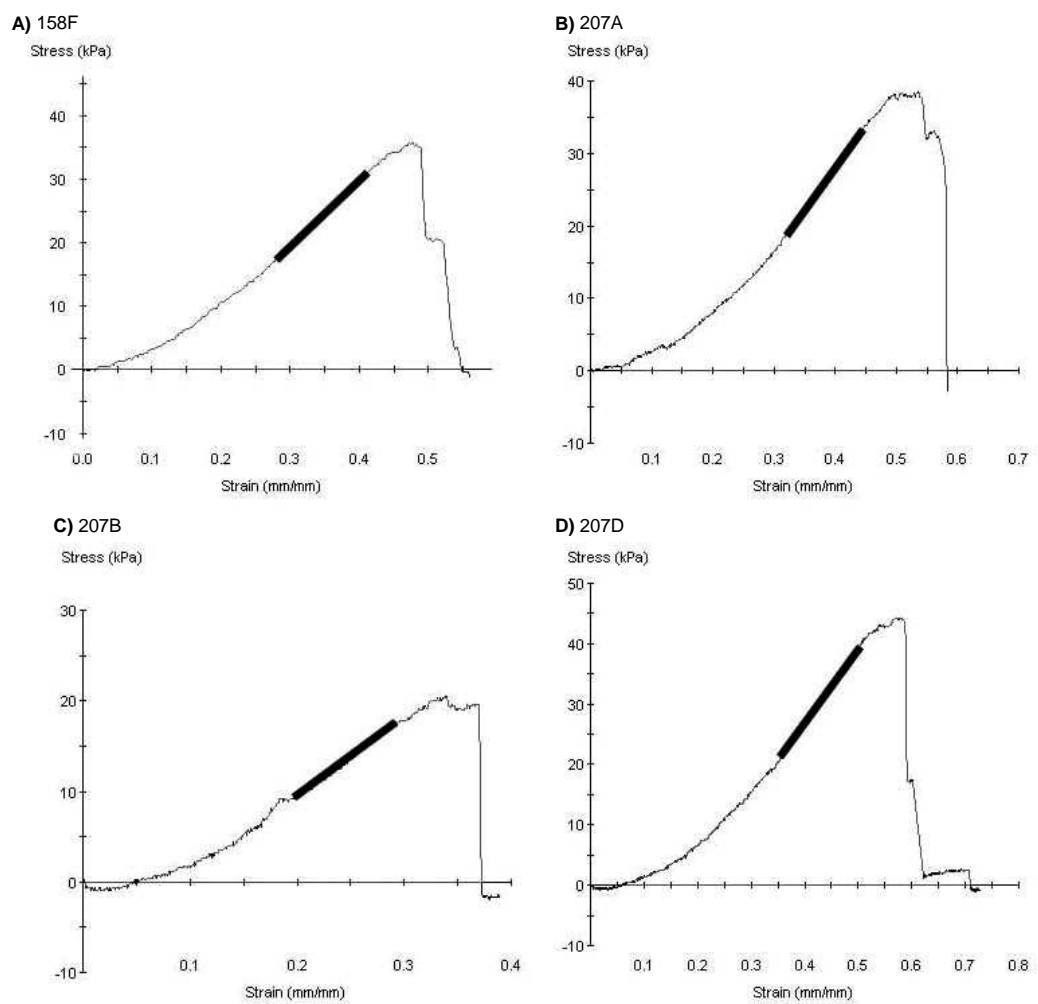

Figure 3.39 Stress versus strain curves for constructs (A) 158F, (B) 207A, (C) 207B, and (D) 207C; the black line indicates the region used for calculation of modulus.

\section{Compression and tension without offset}

Constructs tested with both compression followed by a rest and tension followed by a rest for a total of three times daily for a total of two weeks were the following: 163A, 163B, 163C, 167B, 167C, 169C, 209A, 209B, and 209C. Following the two week stimulation period, constructs were indentation tested, allowed to completely recover, then tensile tested to failure. The stress-strain responses (Figure 3.40) for constructs 163A, 163B, 163C, 167B, and 167C illustrated a toe region, however, constructs $163 \mathrm{~A}$ and $163 \mathrm{C}$ had very little toe region 

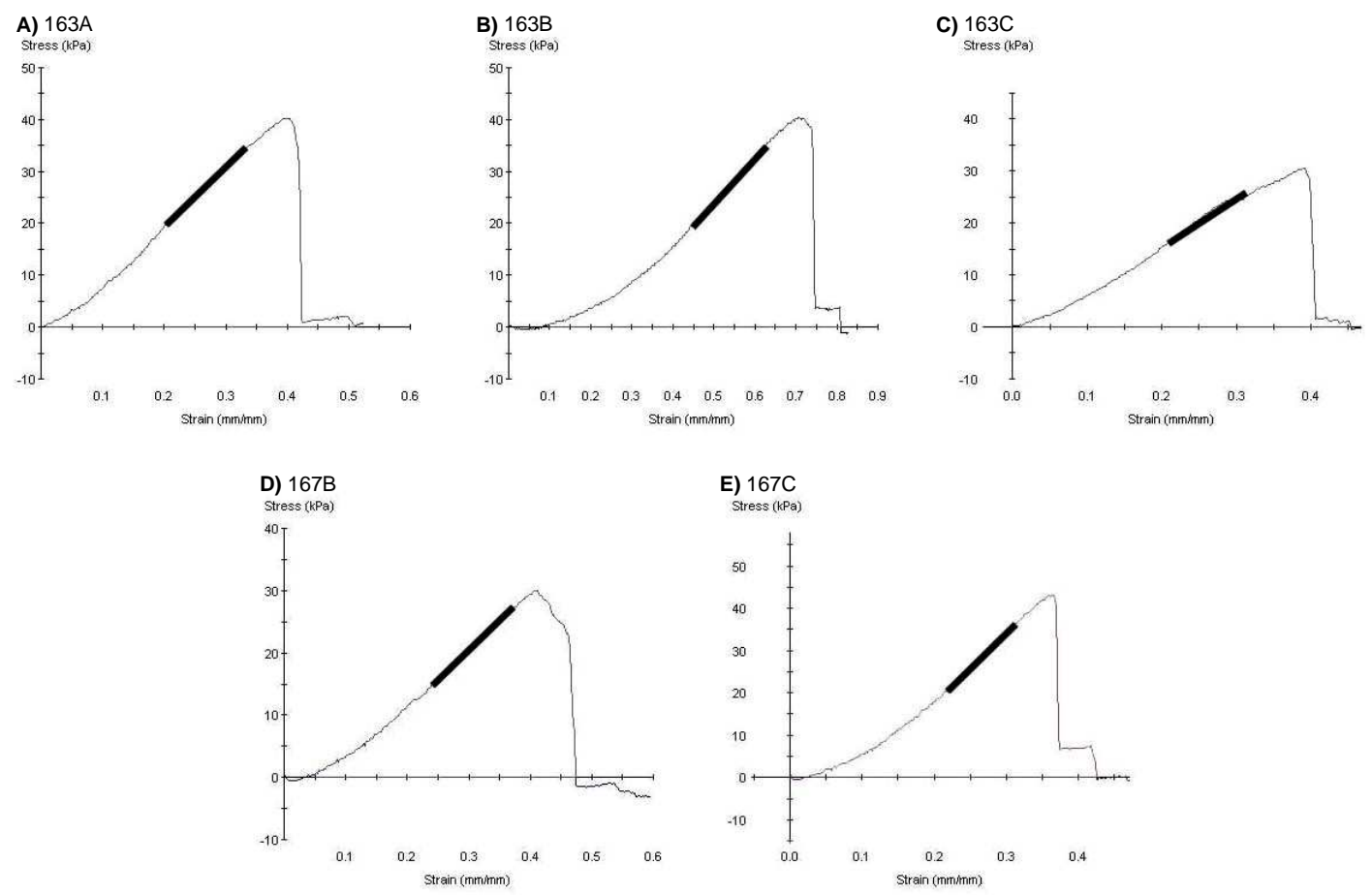

Figure 3.40 Stress versus strain curves for constructs (A) 163A, (B) 163B, (C) 163C, (D) 167B, and (E) 167C; the black line indicates the region used for calculation of modulus.

response. The responses following the toe were proportional for the slope until the peak stress was attained. Upon reaching this peak, the constructs failed immediately and continued until they were completely severed and attained zero stress. From each respective stress-strain curve a modulus of $109.9 \mathrm{kPa}$ for $163 \mathrm{~A}, 80.8 \mathrm{kPa}$ for $163 \mathrm{~B}, 84 \mathrm{kPa}$ for $163 \mathrm{C}, 95.2 \mathrm{kPa}$ for $167 \mathrm{~B}$, and $166.6 \mathrm{kPa}$ for $167 \mathrm{C}$ was calculated.

For constructs 167C, 209A, 209B, and 209C their stress-strain (Figure 3.41) responses followed a path with a toe region that quickly changed to linear. Constructs 209A and 209C continued their proportional response until the peak stress was achieved. However, 167C and 209B had some yielding prior to the peak. As the stress peaked, the constructs failed and continued to decrease in stress until zero stress and complete rupture occurred. From the linear 
portion of each curve the modulus was determined as $139.6,134,138.8$, and $156.7 \mathrm{kPa}$, respectively, for 167C, 209A, 209B, and 209C.
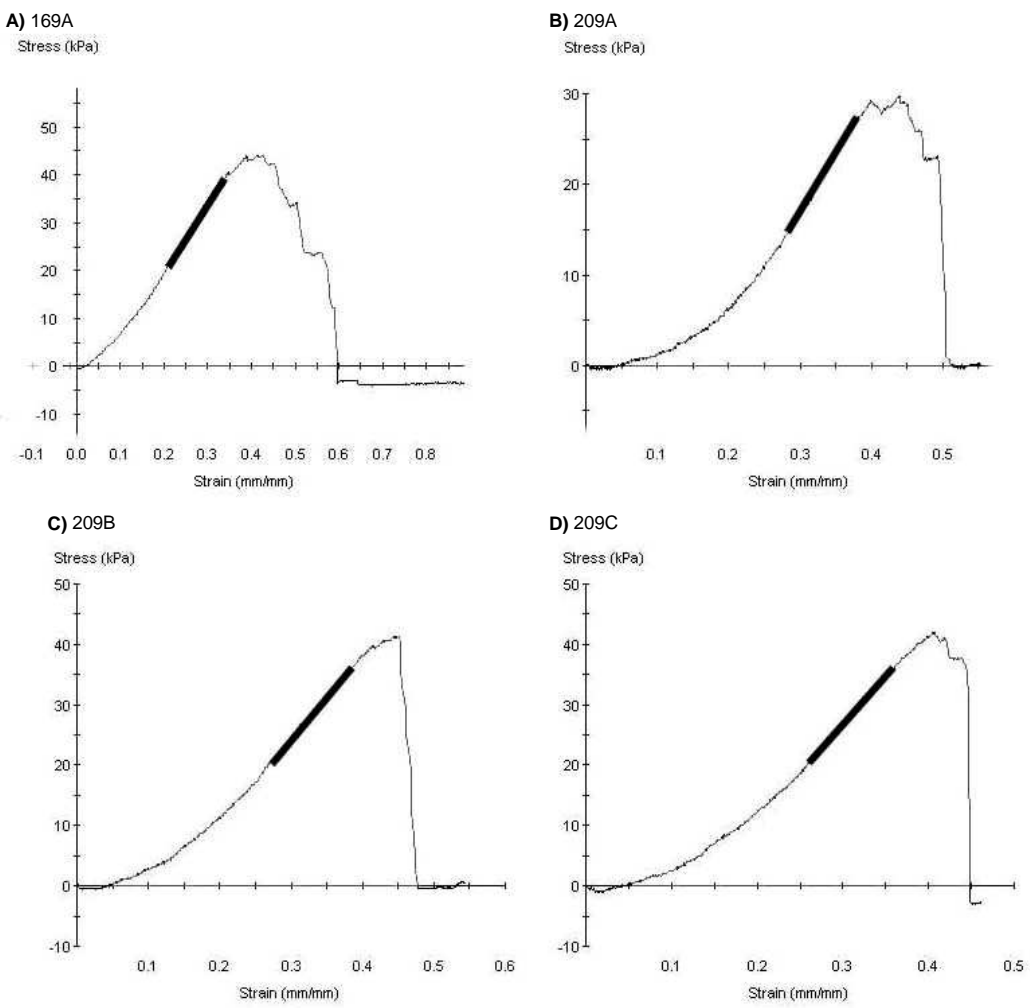

Figure 3.41 Stress versus strain curves for constructs (A) 169C, (B) 209A, (C) 209B, and (D) 209C; the black line indicates the region used for calculation of modulus.

\section{Compression and Tension with offset}

Constructs 184A, 184B, 184C, 186A, 186C, 188A, 205B, 205C, and 205D were stimulated with compression with an offset then rested, followed by tension with an offset, then rest. The stimulation with offset occurred for a total of three times daily over a two week time period. After indentation testing was completed and constructs recovered completely, samples were tensile tested until failure.

The stress versus strain curves (Figure 3.42) for constructs 184A, 184B, 184C, 186A, and 186C exhibited a toe region, which was small for construct $184 \mathrm{~B}$, that changed over to a linear 
portion as stress increased. Some yield in the constructs occurred prior to reaching the peak. Once the peak was reached, the constructs progressively failed until all attachment was severed and the stress reduced to zero. From the curve data, the modulus was calculated as $108.3 \mathrm{kPa}$ for $184 \mathrm{~A}, 91.8 \mathrm{kPa}$ for $184 \mathrm{~B}, 106.6 \mathrm{kPa}$ for $184 \mathrm{C}, 95.3 \mathrm{kPa}$ for $186 \mathrm{~A}$, and $54.5 \mathrm{kPa}$ for $186 \mathrm{C}$.
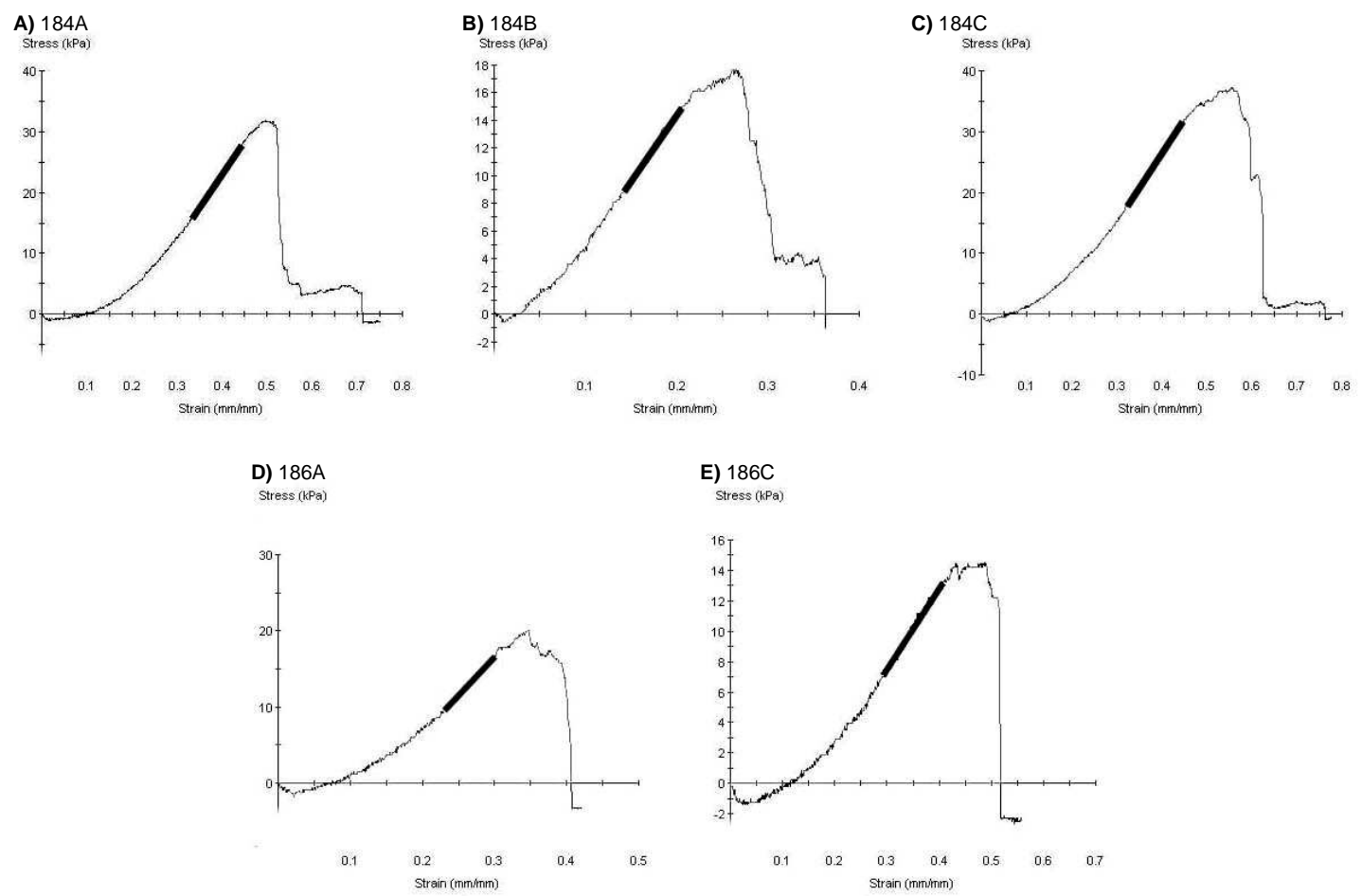

$\begin{array}{llcr}0.1 & 0.2 & 0.3 & 0.4 \\ & & \text { Strain (mnmum) }\end{array}$

Figure 3.42 Stress versus strain curves for constructs (A) 184A, (B) 184B, (C) 184C, (D) 186A, and 186 (C); the black line indicates the region used for calculation of modulus.

The tension tests for constructs 188A, 205B, 205C, and 205D showed stress-strain responses (Figure 3.43) with a toe region, although 188A was small, followed by proportional response almost up to the peak. Some yielding occurred prior to the peak for all constructs and at the peak the constructs ruptured, decreased rapidly in stress, and eventually there was 

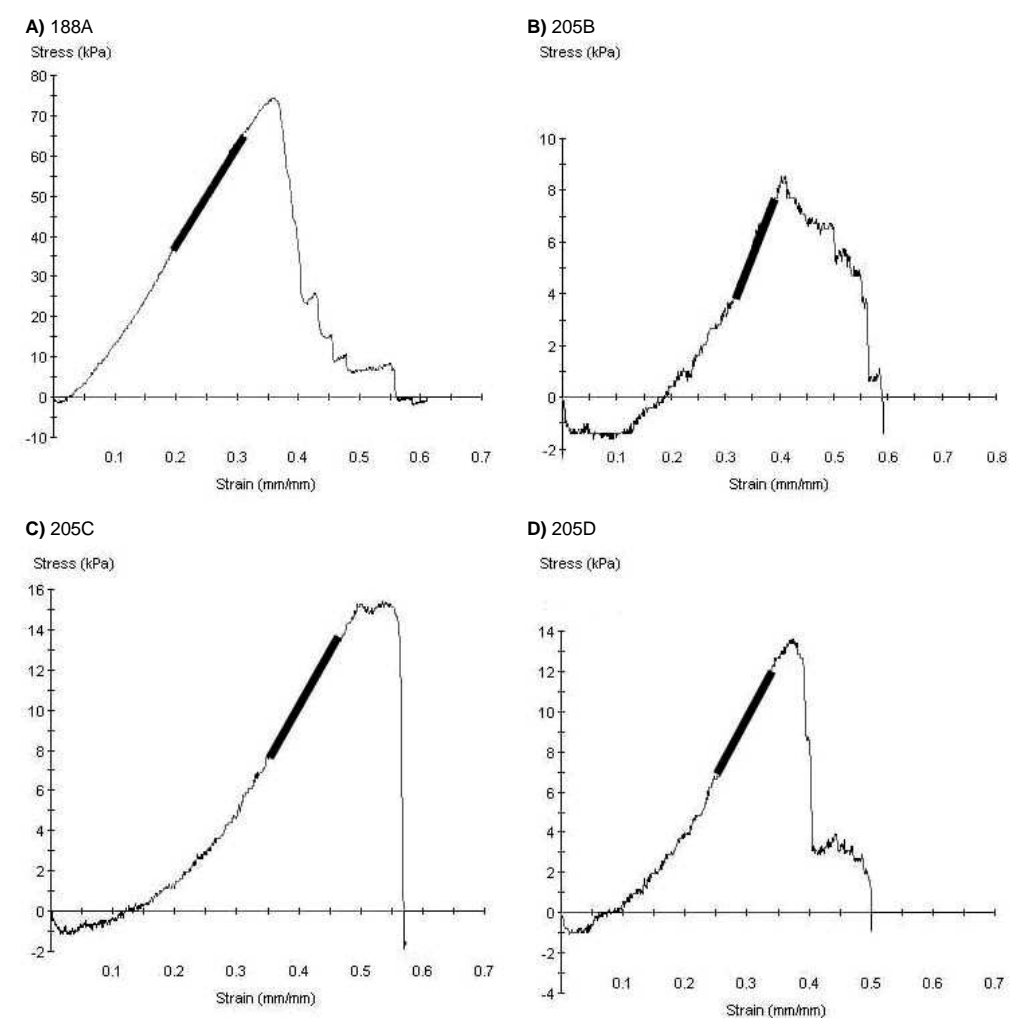

D) $205 \mathrm{D}$
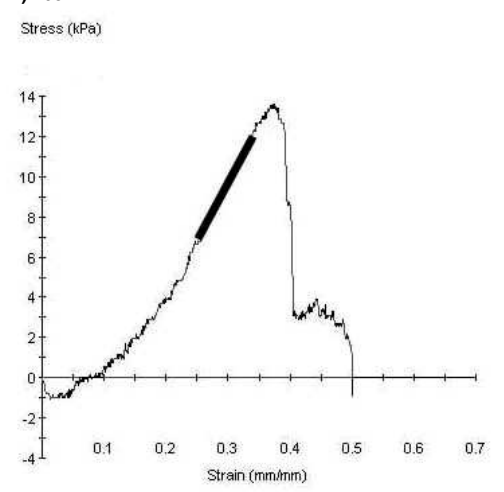

Figure 3.43 Stress versus strain curves for constructs (A) 188A, (B) 205B, (C) 205C, and (D) 205D; the black line indicates the region used for calculation of modulus.

complete failure with a zero stress state. From the linear portion of each stress-stain response the modulus for $188 \mathrm{~A}$ was $257 \mathrm{kPa}, 205 \mathrm{~B}$ was $36.7 \mathrm{kPa}, 205 \mathrm{C}$ was $48.3 \mathrm{kPa}$, and 205D was 59 $\mathrm{kPa}$.

\section{$\underline{\text { Tension only }}$}

Constructs 178A, 178C, 181A, 197A, 197B, 198B, 208A, 208B, and 208C were cultured for 1 week statically, then place in a bioreactor for application of $10 \%$ cyclic tension 3 times daily with a rest period in between stimulation. Constructs were cultured for 2 weeks and tensile tested after recovery from indentation testing.

For constructs 178A, 178C, 181A, 197A, and 197B their response for the stress-strain curve (Figure 3.44) showed an initial toe region that changed to a proportional response as the stress 
increased and continued to rise until yielding began shortly prior to the peak. Further failure occurred at the peak and the stress decreased somewhat before a majority of the damage occurred and the stress rapidly returned to zero as the remaining construct severed. The results of the linear portion of the curve produced a modulus of 41.5, 43, 104, 67.1, and $104 \mathrm{kPa}$ for 178, 178C, 181A, 197A, and 197B, respectively.
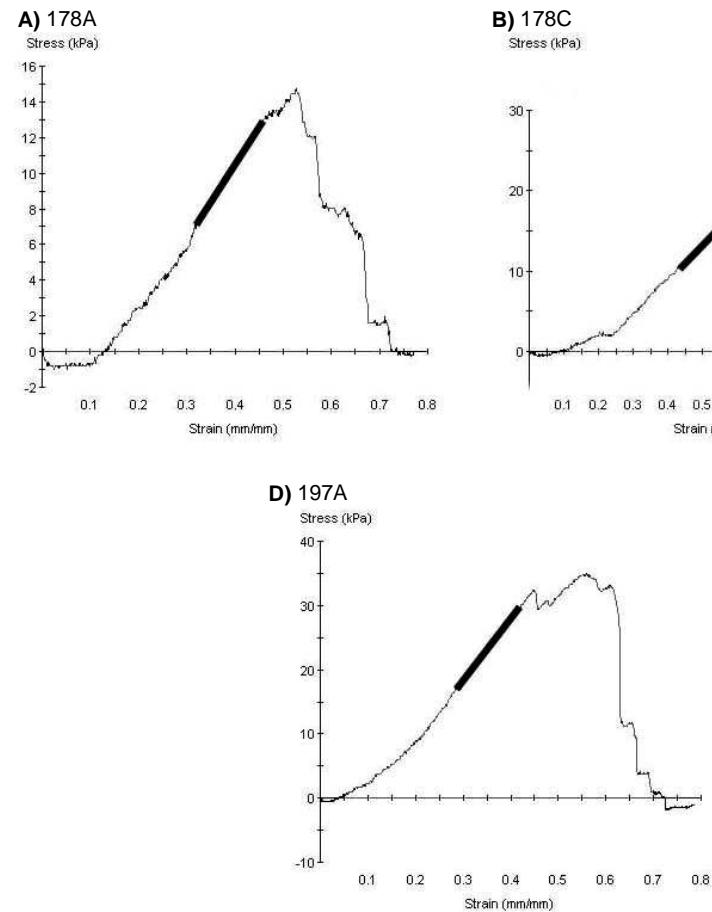

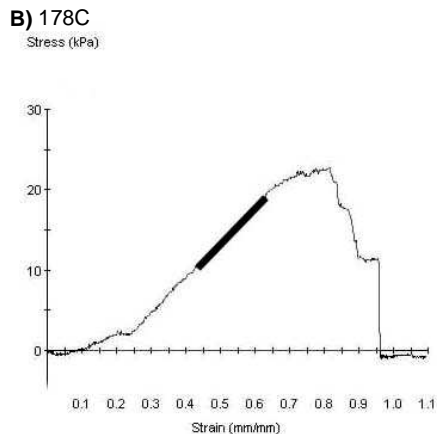

C) $181 \mathrm{~A}$

Stress (kPa)

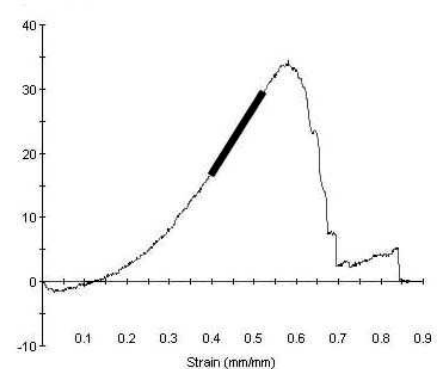

E) 197B

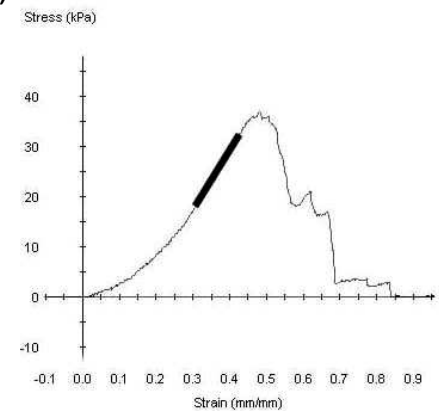

Figure 3.44 Stress versus strain curves for constructs (A) 178A, (B) 178C, (C) 181A, (D) 197A, and (E) 197B; the black line indicates the region used for calculation of modulus.

For constructs 198B, 208A, 208B, and 208C the data exhibited a response for each that began with a toe region for the stress-strain curve (Figure 3.45) and became linear as the stress increased. However, there was some failure prior to the peak stress for all constructs except 198A, which instantly failed at the peak. For the remaining constructs, the stress continued to increase and at the peak they ruptured. Afterwards, the stress quickly declined as the constructs continued to tear until complete failure occurred and zero stress obtained. Analysis of the linear 
portion of the stress-strain curve gave a modulus of $104.8 \mathrm{kPa}$ for $198 \mathrm{~A}, 100.1 \mathrm{kPa}$ for $208 \mathrm{~A}$, 110.5 for $208 \mathrm{~B}$, and 110.7 for $208 \mathrm{C}$.
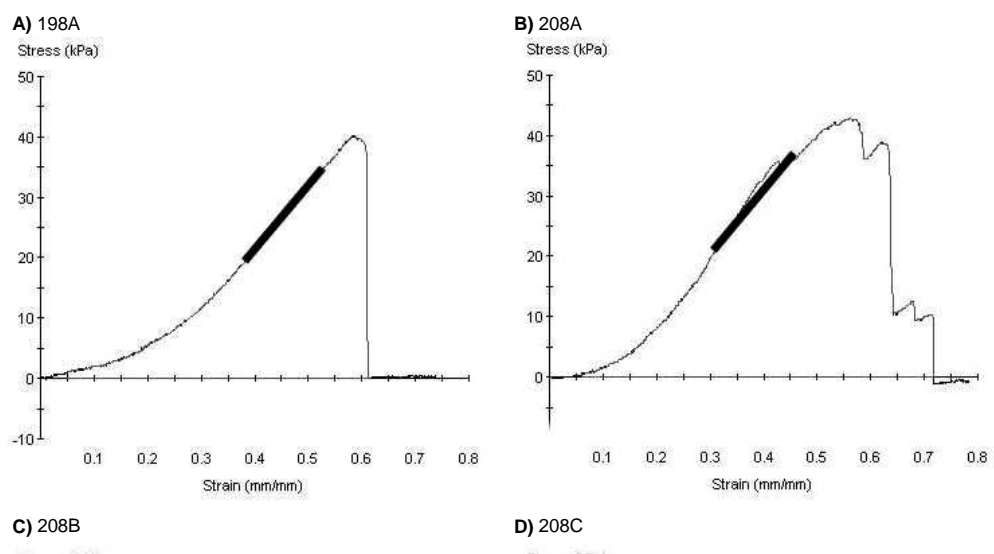

Stress ( $\mathrm{KPa}$

D) $208 \mathrm{C}$

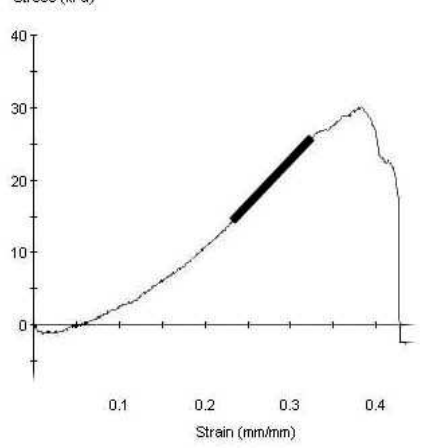

Stress (kPa)

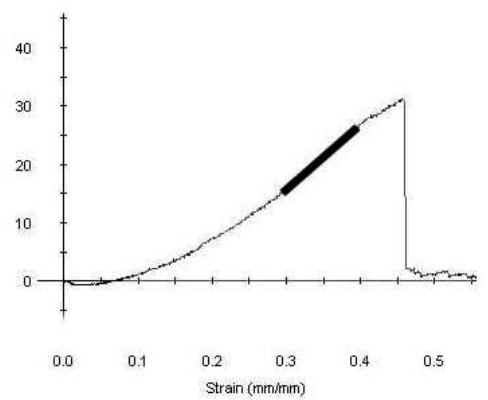

Figure 3.45 Stress versus strain curves for constructs (A) 198A (B) 208A, (C) 208B, and (D) 208C; the black line indicates the region used for calculation of modulus.

Some of the constructs in each group appeared negative in stress at the beginning of the test, which was due to the sensitivity of the $100 \mathrm{~N}$ load cell at small loads. Table 3.2 shows a summary of tensile data for all tested constructs with values for cross-sectional area, peak load, peak stress, and modulus. 


\begin{tabular}{|c|c|c|c|c|c|}
\hline Specimen & Stimulation & $\begin{array}{c}\text { Cross- } \\
\text { sectional } \\
\text { Area } \mathrm{mm}^{\wedge} 2\end{array}$ & $\begin{array}{c}\text { Peak } \\
\text { Load (N) }\end{array}$ & $\begin{array}{l}\text { Peak } \\
\text { Stress } \\
\text { (kPa) }\end{array}$ & $\begin{array}{l}\text { Modulus } \\
\quad(\mathrm{kPa})\end{array}$ \\
\hline $156 \mathrm{~A}$ & None & 5.23 & 0.09 & 17.3 & 60.3 \\
\hline $156 \mathrm{~B}$ & None & 5.55 & 0.12 & 20.9 & 69.1 \\
\hline $157 \mathrm{C}$ & None & 5.25 & 0.16 & 30.9 & 71.2 \\
\hline 157D & None & 6.22 & 0.15 & 24.8 & 85.0 \\
\hline $158 \mathrm{E}$ & None & 3.20 & 0.20 & 43.8 & 138.0 \\
\hline $158 \mathrm{~F}$ & None & 4.88 & 0.18 & 35.8 & 103.0 \\
\hline $207 A$ & None & 5.43 & 0.21 & 38.5 & 117.0 \\
\hline 207B & None & 4.59 & 0.09 & 20.5 & 85.0 \\
\hline \multirow[t]{3}{*}{$207 C$} & None & 5.07 & 0.22 & 44.2 & 122.1 \\
\hline & Mean & 5.05 & 0.16 & 30.7 & 94.5 \\
\hline & Stdev & 0.83 & 0.05 & 10.3 & 26.9 \\
\hline $163 \mathrm{~A}$ & Both no offset & 6.08 & 0.25 & 40.0 & 109.9 \\
\hline 163B & Both no offset & 6.61 & 0.27 & 40.4 & 80.8 \\
\hline $163 C$ & Both no offset & 7.18 & 0.24 & 34.0 & 84.0 \\
\hline 167B & Both no offset & 5.72 & 0.17 & 30.1 & 95.2 \\
\hline $167 \mathrm{C}$ & Both no offset & 4.41 & 0.19 & 43.1 & 166.6 \\
\hline $169 \mathrm{C}$ & Both no offset & 6.61 & 0.21 & 32.4 & 139.6 \\
\hline $209 A$ & Both no offset & 6.61 & 0.20 & 29.8 & 134.0 \\
\hline 209B & Both no offset & 6.48 & 0.27 & 41.3 & 138.8 \\
\hline \multirow[t]{3}{*}{$209 C$} & Both no offset & 5.82 & 0.24 & 41.9 & 156.7 \\
\hline & Mean & 6.17 & 0.22 & 36.7 & 112.7 \\
\hline & Stdev & 0.80 & 0.03 & 5.2 & 34.0 \\
\hline $184 \mathrm{~A}$ & Both with offset & 6.36 & 0.20 & 31.9 & 108.3 \\
\hline 184B & Both with offset & 5.58 & 0.10 & 18.0 & 91.8 \\
\hline $184 C$ & Both with offset & 6.59 & 0.25 & 37.3 & 106.6 \\
\hline $186 \mathrm{~A}$ & Both with offset & 5.31 & 0.03 & 19.0 & 95.3 \\
\hline $186 C$ & Both with offset & 4.84 & 0.86 & 20.0 & 54.5 \\
\hline $188 \mathrm{~A}$ & Both with offset & 5.42 & 0.23 & 86.0 & 257.0 \\
\hline 205B & Both with offset & 4.92 & 0.04 & 8.5 & 36.7 \\
\hline $205 C$ & Both with offset & 6.09 & 0.09 & 15.4 & 48.3 \\
\hline \multirow[t]{3}{*}{ 205D } & Both with offset & 5.15 & 0.07 & 13.6 & 59.0 \\
\hline & Mean & 5.59 & 0.21 & 27.7 & 95.3 \\
\hline & Stdev & 0.63 & 0.26 & 23.6 & 66.2 \\
\hline $178 \mathrm{~A}$ & Tension only & 6.22 & 0.09 & 14.7 & 41.5 \\
\hline $178 \mathrm{C}$ & Tension only & 6.30 & 0.14 & 22.7 & 43.0 \\
\hline $181 \mathrm{~A}$ & Tension only & 4.92 & 0.17 & 34.3 & 104.0 \\
\hline $197 A$ & Tension only & 6.79 & 0.24 & 35.0 & 67.1 \\
\hline 197B & Tension only & 7.07 & 0.26 & 36.0 & 104.0 \\
\hline 198B & Tension only & 6.70 & 0.27 & 40.0 & 104.8 \\
\hline $208 A$ & Tension only & 6.34 & 0.27 & 42.9 & 100.1 \\
\hline 208B & Tension only & 6.91 & 0.21 & 30.1 & 110.5 \\
\hline \multirow[t]{3}{*}{$208 C$} & Tension only & 6.81 & 0.21 & 31.1 & 110.7 \\
\hline & Mean & 6.45 & 0.21 & 31.9 & 87.3 \\
\hline & Stdev & 0.65 & 0.06 & 8.7 & 28.7 \\
\hline
\end{tabular}

Table 3.2 Data summary for all tension tested constructs.

The statistical analysis for cross-sectional area (Figure 3.46) showed a significant difference between all groups $(\mathrm{p}<0.0013)$. Additional comparisons showed the groups both no offset and tension were different from Day 0. There were no other differences between groups. 


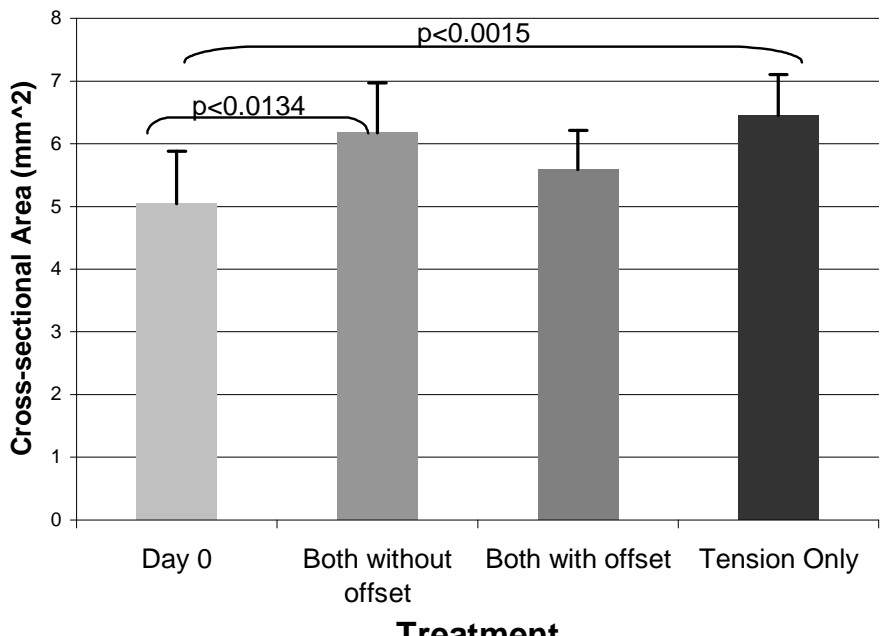

Figure 3.46 Cross-sectional area for each of the groups.

The statistical analysis for peak load (Figure 3.47) with a one-way ANOVA showed the means of the groups were significantly different $(\mathrm{p}<0.0035)$. Further comparison showed there was a significant difference between the following groups: stimulation with both compression and tension without offset, stimulation with both compression and tension with offset, and tension only. Day 0 was not significantly different from any of the groups. 


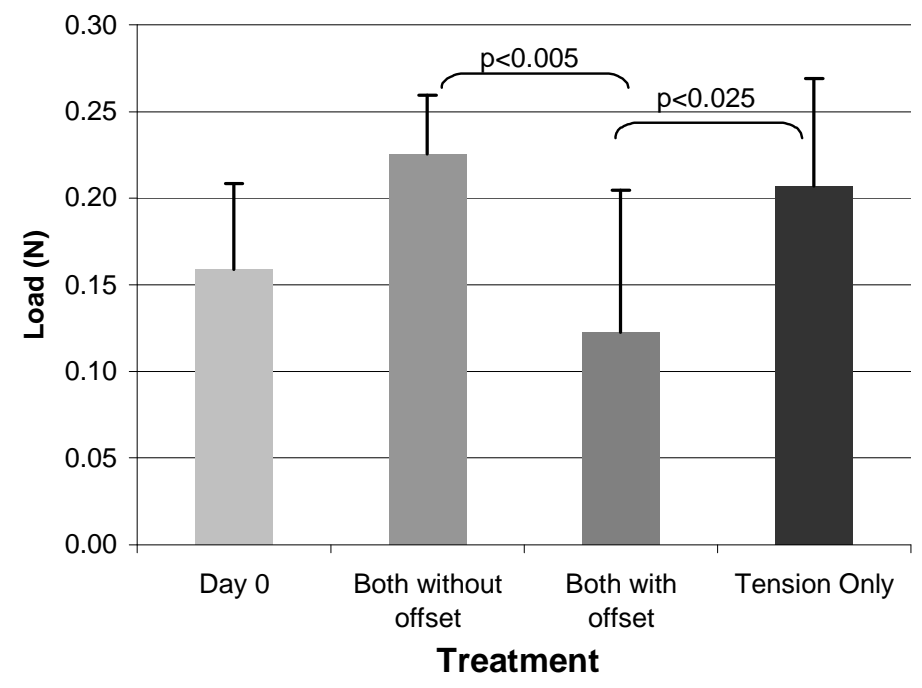

Figure 3.47 Peak load for each of the groups.

The analysis of peak stress (Figure 3.48) with a statistical one-way ANOVA showed no significant difference between any of the group means.

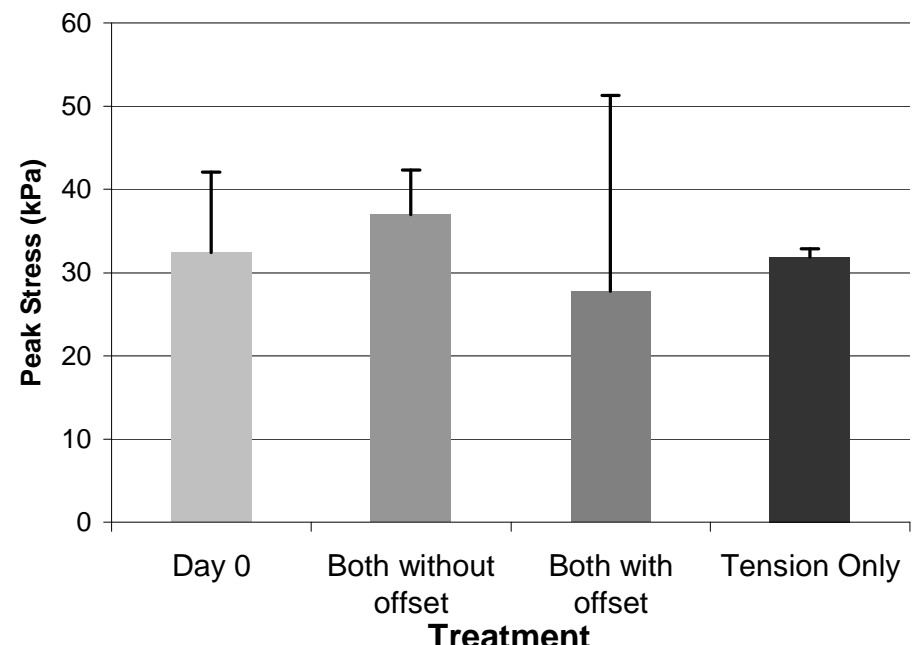

Figure 3.48 Peak stress for each of the groups.

Finally, for evaluation of the modulus (Figure 3.49) a one-way ANOVA showed there was no significant difference between any of the evaluated groups. 


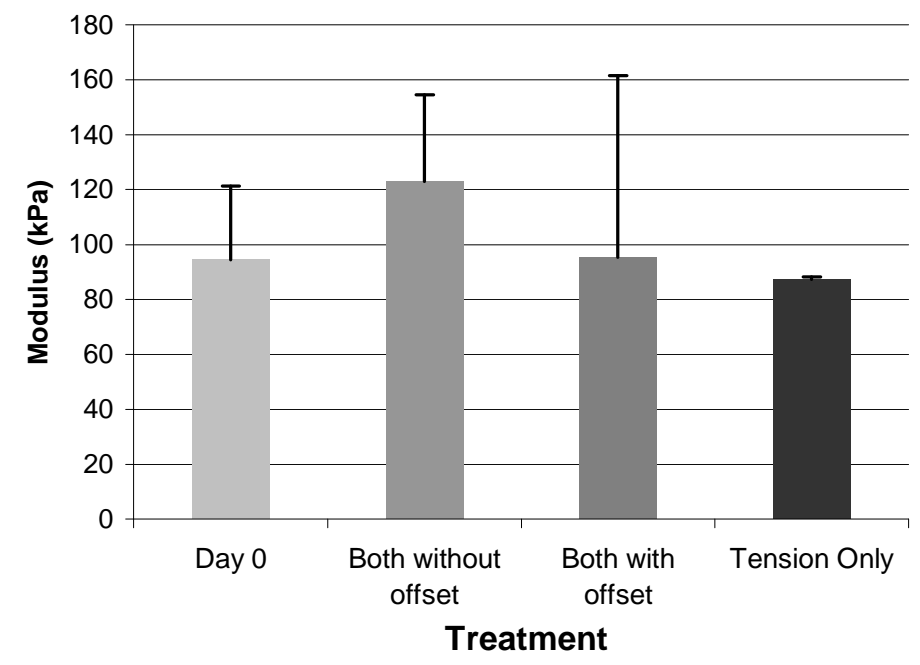

Figure 3.49 Tensile modulus for each of the tested groups.

\subsection{WESTERN BLOT}

Western blot analysis was performed on 3 constructs from each of the following groups: Day 0 (constructs 206A, 206B, and 206C), compression and tension without offset (constructs 200A, 200B, and 200C), compression and tension with offset (203B, 203C, and 203D), and tension only (199A, 199B, and 199C). Figure 3.50 shows images of gels 1 and 2 with both the major and minor aggrecan band and GAPDH for normalization ${ }^{77}$.

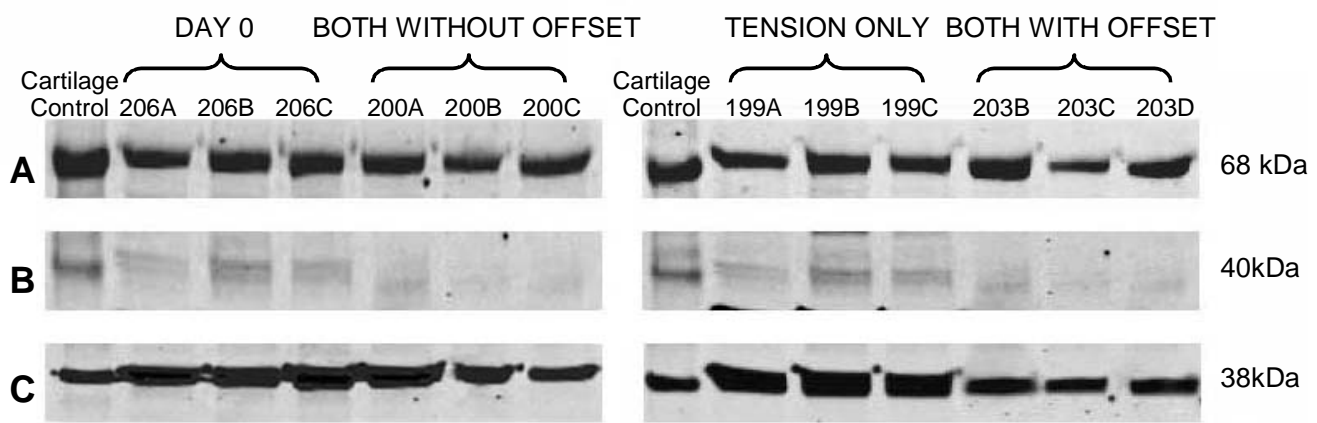

Figure 3.50 Picture of western blot gels after treatment with antibody to aggrecan was completed A) major band of aggrecan, B) minor band of aggrecan, and C) GAPDH (loading control). 
The major aggrecan band with a molecular weight of $68 \mathrm{kDa}$ was found in all samples and the cartilage control, although overall the stimulations of both with offset and tension only constructs showed less intensity, which indicated that there stimulation algorithms did not produce as much higher band aggrecan fragment as the Day 0 and both without offset. However, when normalized to the loading control both with offset showed a higher expression (Figure 3.51).

For the minor aggrecan band with a molecular weight of $40 \mathrm{kDa}$ there was less intensity in the both without offset and with offset bands. Though, when the data was normalized to the intensity of the GAPDH (Figure 3.52) the tension and both without offset were actually lower in the amount in each construct than the Day 0 and with offset, which indicated that these stimulations produced less of the lower band aggrecan fragment.

Evaluation of the normalized data to the GAPDH loading control with a one-way ANOVA showed that there was a significant difference between the means $(\mathrm{p}<0.00189)$. Posthoc comparison with a Tukey-Kramer test revealed Day 0 was significantly different from all groups. There was no significant difference between the remaining groups. Figure 3.51 is a graph of the upper band of aggrecan means. 


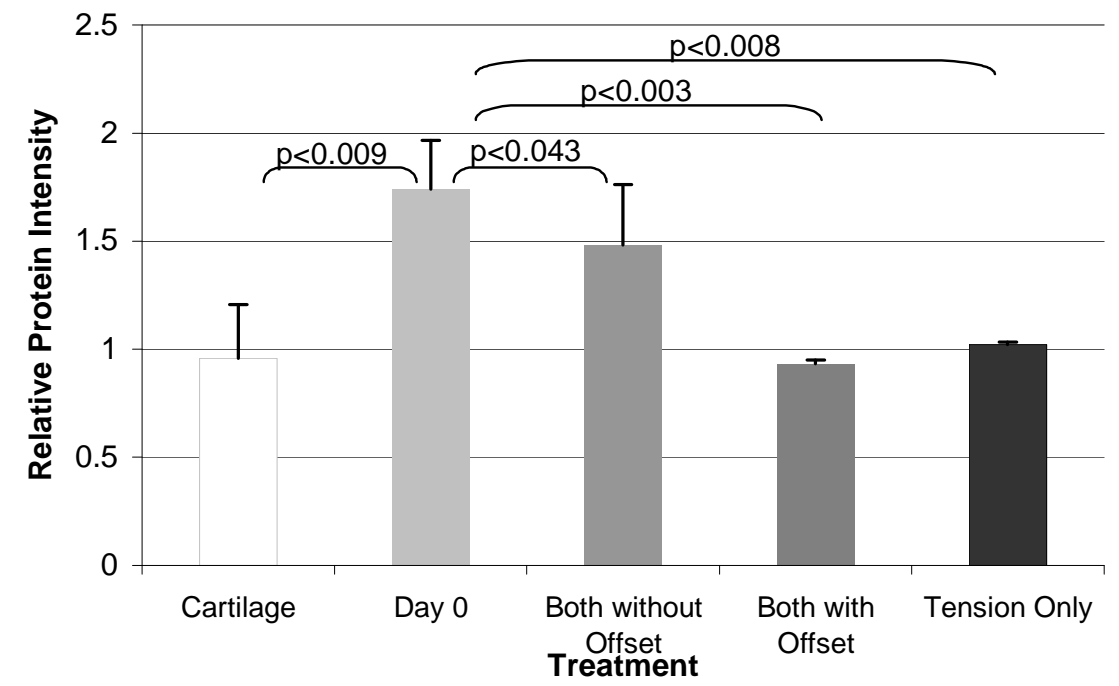

Figure 3.51 Relative protein intensity for aggrecan from the upper band for human articular cartilage and all tested groups normalized to the GAPDH band.

The results from the statistical analysis for the lower band of aggrecan showed there was a significant difference between the means $(\mathrm{p}<0.00287)$ with a one-way ANOVA (Figure 3.52). Further post-hoc testing with a Tukey-Kramer comparison showed a significant difference between Day 0 and all other groups, except the both without offset group. Additionally, there was no significant difference between any of the remaining groups. 


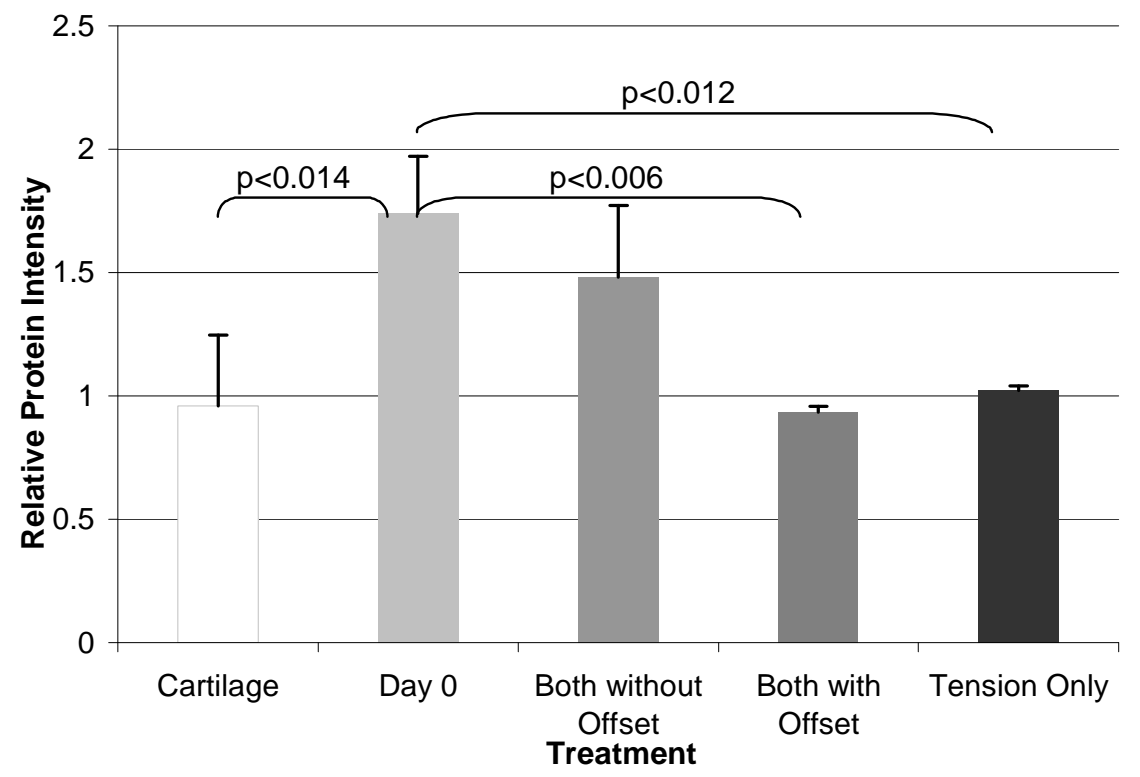

Figure 3.52 Relative protein intensity for aggrecan from the lower band for human articular cartilage and all tested groups normalized to the GAPDH band. 


\section{CHAPTER 4 DISCUSSION}

\subsection{BRIEF OVERVIEW}

This work as well as many others in the literature has demonstrated the ability to improve the structural and functional characteristics of tissue engineered constructs as possible tissue replacement products through the application of mechanical stimulation algorithms. This work however is the first to focus these efforts in developing a superficial tangential zone product for articular cartilage repair. We hypothesized that in using MSCs with collagen type I scaffolds for our constructs, we would have an abundant source of cells that would produce cartilage-like components when treated with chondrogenic media. We further hypothesized that application of compression would increase production of characteristics seen in the superficial tangential zone, such as proteoglycans and matrix production along with improved mechanical properties. Additionally, further stimulation with tension loading would manipulate cell growth directionally aligned. Our results illustrated a variety of promising improvements to tissue constructs with matrix production, mechanical properties, and cell/matrix arrangement that may eventually lead to long-term cartilage repair by replacement of the superficial tangential zone layer at onset of surface damage or as a layer for full thickness defects. The results of the different stimulation algorithms evaluated are examined below.

\subsection{DAY 0}

Constructs cultured without mechanical stimulation showed some increase in matrix as compared to scaffold alone due to the cues provided the cells by the chondrogenic media. Additionally, cell distribution was visible throughout the collagen scaffold and exhibited a round morphology commonly seen in three-dimensional cultures ${ }^{40,78,79}$. In the present study, there was 
some alcian blue staining present in the Day 0 scaffolds, but not a great amount. These findings are consistent with previous work done by Connelly et al. that found an increased content of GAGs in as little as 1 week on static cultures ${ }^{79}$. In Day 0 constructs, GAGs were present in higher concentration on the surface than deeper within the constructs, which may be due to static culture conditions not providing as much nutrient exchange to the cells in the interior of the construct. Free-swelling static cultures were found to exhibit fibers with more of a homogeneous matrix ${ }^{79}$.

SEM micrographs of the Day 0 constructs showed some matrix deposition and visible cells. The matrix was more organized than scaffold alone, but did not seem as organized at stimulated constructs. The deposition of matrix materials was evident, which may be both collagen types I and II, as this was not assessed in our study ${ }^{80}$. Caterson et al. detected cartilage specific traits of collagen type II and IX and aggrecan in 1 week static culture of MSCs seeded on polylactide/alginate amalgam ${ }^{65}$. Additionally, our results are consistent with previous findings using poly (DL-lactide-co-glycolide) (PLGA) sponge scaffolds to culture rat chondrocytes with use of a dynamic seeding method to infiltrate cells throughout when visualized with SEM ${ }^{81}$. These support our results of MSCs in collagen type I scaffold producing cartilage-like components with aggrecan and increased matrix. In a study by Stok et al. with human MSCs seeded on hyaluronan based-scaffolds whereby initial seeding to 1 week in static culture increased expression of aggrecan and collagen type I, there was very little increase in collagen type $\mathrm{II}^{82}$. Additionally, there was some increase in the amount of cells present from seeding to 1 week. Our constructs were not compared on the initial seeded constructs since cell infiltration was seen throughout the depth of the construct after a week in static culture. 
Cell morphology in our Day 0 static constructs were rounded and their alignment overall tended to angle more in a random than patterned, although they had a higher relative frequency of cell angle near 80 degrees. There may be some effect produced by the collagen type I scaffolds used in this study that induce them to grow more in one direction. Previous studies have confirmed a preferential alignment for cells grown on nanofibrous oriented electrospun scaffold in static culture ${ }^{83}$. In our experiment, all scaffolds were cut exactly the same way from the KN scaffolds and the histology and SEM was performed always using the long axis as reference. Since the scaffolds specifics are proprietary, there is no information about the fiber design that shows a tendency to grow cells in a specific configuration. In previous studies, scaffold patterning can influence cells and fiber deposition directionally ${ }^{84}$. Static culture longer than 1 week may produce different alignment effects of more random design or cells may continue to align perpendicular to a particular axis inherent in the scaffold. Visual examination of the KN collagen type I scaffolds without cells via_histology and SEM does not show any clear pattern of the collagen fibers.

Mechanical properties obtained from indentation testing for Day 0 constructs had properties below that found in normal articular cartilage. Indentation of normal cartilage demonstrates an instantaneous compressive load with a tissue response of instant deformation followed by slow increased deformation over time ${ }^{85}$. Our Day 0 samples exhibited this behavior, however they progressed to their final deformation quickly and reach equilibrium in a short time, indicating less strength in the tissue. The results from indentation show an aggregate modulus of $0.018 \mathrm{MPa}$ that is below native cartilage, which is usually in the range of 0.5 to $0.9 \mathrm{MPa}{ }^{15}$. Since aggregate modulus indicates the stiffness of the construct and is directly related to 
proteoglycan content, it fits that Day 0 aggregate modulus is the lowest as supported by the results obtained in histology showing limited GAG content in the depths of the construct.

Huang et al. found the human humeral head superficial cartilage to have a permeability of $1.0 \times 10^{-14} \mathrm{~m}^{4} / \mathrm{Ns}$; Day 0 constructs had a permeability of $2.71 \times 10^{-13} \mathrm{~m}^{4} / \mathrm{Ns}{ }^{86}$. With this higher permeability, a greater amount of fluid flows through the construct when the load was applied, which coincides with the deformation seen in the indentation graphs and the speed at which equilibrium was reached. The lack of GAGs present to assist in resistance of fluid exudation could be responsible for a higher permeability. As for Poisson's ratio, the value was determined at 0.16 for this group, which for normal articular cartilage, evaluated in many studies produced a variety of ranges; the majority of these studies show cartilage at value of 0.5 at the equilibrium. A Poisson's ratio near 0.5 means when the specimen deforms over time and when reaches equilibrium, becomes incompressible. Mak et al. ${ }^{76}$ modeled Poisson's ratio as 0.5 , Hori et al. ${ }^{87}$ determined Poisson's ratio as $0.42-0.49$, Korhonen et al. ${ }^{88}$ determined values at $0.3-0.8$ and Athanasiou et al. determined the value as 0.197. Importantly, some of the varying parameters in these studies used to determine Poisson's ratio range included testing environment, boundary conditions, and area of articular surface ${ }^{15}$. Our overall mechanical testing results for Day 0 constructs are lower than any of the predicted values, indicating at equilibrium these constructs are not incompressible and have inferior qualities to native cartilage.

The tensile properties of Day 0 constructs exhibited a stress strain curve similar to native cartilage. However, the results showed inferior properties of modulus $94.5 \mathrm{kPa}$. Native articular cartilage has been shown in tensile experiments to exhibit modulus ranges of 5-50 MPa ${ }^{86}$. Due to the short culture time of the Day 0 constructs inferior modulus was expected. MSCs seeded in hydrogels and cultured statically for 56 days in chondrogenic media achieved a modulus of 405 
$\mathrm{kPa}{ }^{37}$. It follows that extended culturing time of Day 0 constructs could improve tensile properties.

Western Blot results show that Day 0 constructs exhibited the highest amount compared to all tested construct and cartilage of aggrecan content in both the major and minor aggrecan bands. This is consistent with previous data that shows MSCs cultured for at least 6 days in 3D scaffold with chondrogenic media have been shown to exhibit increased expression of aggrecan genes ${ }^{80}$. Additionally, Emin et al. demonstrated that after 1 week in static culture, rat chondrocytes seeded in PLGA scaffold exhibit aggrecan staining ${ }^{81}$. When we compare our Day 0 results of our western blot with that of the surface of human articular cartilage, the number was much higher than that of native articular cartilage superficial tangential zone. In examining previous studies, the superficial tangential zone contains the least amount of proteoglycans such as aggrecan compared to the underlying zones ${ }^{89,90}$. Alcian blue staining was not as strong as the other groups we tested. These finding suggest some possibilities for the high aggrecan content that contradicts the histological results that the frozen slices are very fragile and there may have been some loss of GAG from the slides or the GAGs did not pick up the staining as they should in the deeper section from procedural error. Day 0 constructs may have a greater presence of integrins to break down aggrecan in to fragments than the stimulated constructs and normal cartilage, thereby creating the illusion of a greater amount of aggrecan through the fragments. Previous work has indicated application of compression inhibits aggrecan breakdown by integrins ${ }^{91}$. Additional research that focuses on aggrecan content in both non-stimulated and stimulated constructs is necessary to better understand the role of aggrecan within these constructs. 


\subsection{COMPRESSION AND TENSION WITHOUT OFFSET}

For constructs cultured with both compression and tension without an offset, the histology showed similarities to Day 0 constructs in their distribution of cells throughout the construct. However, the cells seemed more elongated than no stimulation. In previous studies with application of tension, cells seeded in a 3-D scaffold tended to aligned parallel to direction of the applied tension ${ }^{92}$. The stimulated constructs without offset also had more GAGs present further within the scaffold, which may be due to application of compression allowing further nutrient/waste exchange deeper within the construct. Previous studies with application of compression have found increased staining for GAG with application of compression ${ }^{45,93}$. Studies performed with tension on constructs containing bovine MSCs seeded in fibrin gel found increased GAG content with application of cyclic tensile loading evaluated after 12 days in culture $^{79}$. Furthermore, DeWitt et al. found increased GAG precursors after only 24 hours of cyclic tensile strain ${ }^{54}$.

For SEM more cells were visible than in the Day 0 constructs. Terraciano et al. found with live-dead assay that there were more cells present after application of mechanical stimulation than in non-stimulated constructs and found an increase in the amount of aggrecan and collagen type II expression ${ }^{94}$. We had an increase in aggrecan seen in the histology as compared to the Day 0 constructs and in the SEM there was more matrix deposition, although it was not analyzed for type of collagen expression. Terraciano et al. also found an increased daily frequency increased the amount of expression in the cultures, which supports our repeated stimulation of 3 times daily with both compression and tension was an appropriate choice for inducing chondrogenic traits in our tissue constructs ${ }^{94}$. 
The cellular alignment had a highest relative frequency at 10 degrees. It seems the compression and tension without offset group had cells after two weeks of stimulation migrating towards 0 degrees and aligning with direction of application of tension. Average alignment for both compression and tension without offset was 38 degrees. This finding is consistent with a previous study that applied tension to fibroblasts seeded in a collagen gel. The constructs were subjected to cyclic strain and by day 4 found a linear distribution of cells and fibers as seen by histological evaluation ${ }^{95}$. Furthermore, studies with compression also found cells tend to reduce their strain by orienting with a preferential direction ${ }^{96}$. Cells generally shape themselves in a way that reduces the amount of strain they endure. Application of mechanical forces aid cell cultures in producing a preferential alignment. Our study group with application of both compression and tension allowed for the cells to elongate in the direction of applied tension. Unlike all other tested groups, this group had the majority of its cells closest to the direction of tension application. It may be that inclusion of compression along with application of tension, with no offset strain, produces a synergistic affect that was not seen in the other groups that led to the majority of cells in a specific direction. Connelly et al. found preferentially directed fibers parallel to the application of tension in their stimulated constructs ${ }^{79}$.

Application of both compression and tension without offset produced the highest modulus value overall of any of the indentation tested specimens of $0.028 \mathrm{MPa}$. This indicated a greater stiffness than the other tested groups. Additionally, the constructs in this group deformed instantly, but reached their final deformation slower than the Day 0 constructs. The indentation curve-fit results also showed greater permeability in both compression and tension with no offset constructs than normal native cartilage values stated above. Additionally, there was no overall difference between the tested groups, although the general trend in the bar graph showed 
permeability higher than all other tested groups. There was a higher value of Poisson's ratio of 0.32, which is more in the scope of native articular cartilage, although, the experimental results for human adult articular cartilage measurements for Poisson's ratio vary greatly from study to study depending on site evaluated and testing conditions. Again the stimulation without offset seemed to induce a synergistic effect creating a higher modulus and Poisson's ratio compared to the other groups with and without stimulation.

The results for tensile testing of the both compression and tension with no offset resulted in a modulus of $112.7 \mathrm{kPa}$. Although, there was no significant difference in the modulus values between all groups, trends seen in modulus show this group did have the highest value compared to the other groups. The modulus, however, was not in the range of normal articular cartilage mentioned above. It may be, then, that since the modulus was lower than normal cartilage, such strategies as longer culture time could improve the modulus results.

The western blot results showed the highest content of aggrecan after the amount in Day 0 for the compression and tension without offset, although it was not significantly higher than native cartilage in both the major and minor aggrecan bands. The histological staining supports the higher content of aggrecan. In a previous study evaluating repair models, there was increased cell adhesion and more filling of the defect enhanced by treatment with enzymatic solution to decrease the PG content at the defect site ${ }^{97}$. A higher aggrecan content than native cartilage may be a necessary characteristic to survive long term in the in vivo environment from possible breakdown. 


\subsection{COMPRESSION AND TENSION WITH OFFSET}

The histological sections of compression and tension with offset had similar findings to without offset, cell infiltration, rounded cells, and GAGs found throughout its depth. Studies conducted with compression and offset have found increased GAG compared to free-swelling cultures. In a studies completed by Davidson et al. ${ }^{98}$ and Lee et al. ${ }^{45}$, increased GAG content with application of dynamic compressive stimulation during culture that included a static offset. Additionally, fluid flow within the bioreactor may contribute to increased GAG content. Seidel et al. found increased GAG concentration with application of dynamic compression with a static offset and fluid perfusion ${ }^{99}$. Loading with application of both compression and tension with an offset has not been previously evaluated. Over a two week stimulation period there were no visual differences in the histology between the without offset and offset groups. Further studies are need to evaluate the long-term effects of this combination of both compression and tension with offset to see if there are any additional difference with use of offset on both axes.

The SEM results for both compression and tension with offset were similar to that of the without offset results. This finding in the quality of the internal structure suggests that the stimulation with or without offset did not affect the amount of matrix present, cell layout, or GAG without further investigation. The results of histological staining and SEM are almost identical for both testing algorithms that include compression and tension, however, closer examination of the mechanical properties and western blot results show differences revealed between the loading algorithms.

Cell alignment in the compression and tension with an offset was more random than the results from both without offset; however, the results were not similar to Day 0 either. Accordingly, application of offset with both compression and tension affect the cells so they 
were not able to determine or incapable of responding to a particular direction to lessen the effects of strain. This is likely due to the potential impact of offset strain in both the compression and tension directions. Evaluation of offset strain effects on directional growth of cells has not been thoroughly evaluated, however, a study completed with an offset and dynamic compression applied for 7 days induced alignment along loading direction in human MSCs seeded in agarose gel ${ }^{96}$. The compression and tension with offset group, although mostly random in alignment, had a greater tendency for cells at a 44 degree angle to the direction of applied tension. The resultant 44 degree angle is similar to our results obtained with tension only, indicating a synergistic effect to promote cell alignment with both compression and tension without an offset, but with the addition of offset or tension alone it may take longer for the cells to reach a preferential alignment along the axis of applied tension. Studies that include longer culture time with this algorithm are needed to investigate alignment further. .

Indentation results showed properties inferior to native cartilage as in the previously tested groups; however there was improvement over the Day 0 non-stimulated constructs. The modulus was closer in value to that of without offset than any of the other results, although there was no statistical difference found between its modulus and that of Day 0 and tension only. Additional findings on permeability show similar findings to the other groups with inferior permeability values in the $10^{-13} \mathrm{~m}^{4} / \mathrm{Ns}$ range, higher permeability than the range for normal cartilage stated above. Poisson's ratio of 0.23 was lower than native, but a higher value than Day 0 and closer to the range of normal cartilage.

Overall indentation results for the construct group with offset showed an instantaneous deformation, which over time had a greater initial instant deformation that continued to deform a greater amount than the group without offset. These findings suggest that the determined 
properties from evaluation of the construct with creep are inferior to that of the without offset group. Buschmann et al. applied dynamic compression with an offset and found modulus increased and permeability decreased ${ }^{78}$. It may be in the present study, use of tension with an offset on the constructs did not result in an additional benefit to the mechanical properties.

Tensile testing results showed inferior properties for constructs with compression and tension with offset, which was lower than normal articular cartilage. Although not statistically significant, there was a trend send in a higher value of modulus obtained in testing the both with an offset compared to the Day 0 and tension only results. As such, the outcome benefit to the mechanical properties when offset loading is applied in this stimulation algorithm cannot be made without additional study.

Western Blot results showed that constructs stimulated by compression and tension with offset had significantly less content of aggrecan protein than the Day 0 and without offset group, however, the results were similar to that of the native cartilage evaluated, indicating that the static offset stimulation may assist with keeping the cells from over producing the aggrecan content for a STZ replacement construct. However, results contradicted the histology staining, although the increased blue staining may be from proteoglycans other than aggrecan.

\subsection{TENSION ONLY}

Constructs stimulated with tension alone had cells throughout their layers, however, there did not seem to be as abundant. In a previous tension study performed on tendon constructs, decreased cell proliferation was seen in constructs stimulated with cyclic stretching for 60 minutes ${ }^{100}$. Possibly with tension only, there is an increase in cell death due to less nutrient exchange than with application of compression. Although there was a decrease in cell number 
there was still GAG staining present within the construct depths. In tension only studies GAG content was found to increase after stimulation with tension only ${ }^{57,60}$.

For SEM there was some fiber alignment but not as much matrix was seen as in the other stimulation algorithms; more pores were present in the tension only constructs. It may be that compression with or without offset is a necessary component to induce extracellular production, particularly collagen type II. Application of cyclic compressive forces in various studies has seen an increase in the amount of collagen type II in the constructs ${ }^{44,67,94}$.

Tension only stimulated constructs had similar cell distribution to that of both compression and tension with offset stimulation group. Perhaps tension alone did not produce a strain effect large enough to cause the cells to align in the direction of tension or it was too large for the cells to be able to respond to consistently create a mean alignment at a relative angle of 44 degrees. A study that supports the tension only finding was completed on murine stem cells seeded in Kensey Nash collagen I scaffolds and found cells randomly aligned after 14 days of cyclic tensile loading at $2.4 \%$ peak strain applied at 20 seconds of cycling followed by 100 seconds rest for 5 hours daily ${ }^{49,67}$. It could be that the amount of tension strain was not enough to induce preferential alignment with the MSCs seeded in the collagen I scaffold. More research is needed to compare tension application with other scaffold sources and different loading regimens.

The mechanical properties of the tension only constructs showed an aggregate modulus that was not significantly different from Day 0 and both with offset, furthermore, it was much lower than that of normal articular cartilage. As with all the test groups, the permeability values were near $10^{-13} \mathrm{~m}^{4} / \mathrm{Ns}$, which is higher than native cartilage values of $10^{-15} \mathrm{~m}^{4} / \mathrm{Ns}$. Additionally, the tension only group exhibited the lowest Poisson's ratio compared to the other stimulation 
groups, however, it was still higher than Day 0. The indentation data indicated an instantaneous deformation that followed a greater amount of deformation on the way to equilibrium, which was similar to the behavior found in the Day 0 and with offset groups. The behavior of the tension only stimulated constructs is inferior to that obtained from the both without offset group.

Tension testing results showed no difference between any of the groups for modulus. Additionally, the tension data exhibited the lowest value of modulus. All results were lower than native articular cartilage. Further testing is necessary to see if there is a difference in tension properties. The western blot analysis showed the aggrecan content for both the major and minor fragments in tension only constructs was similar to that obtained for the with offset stimulation and the cartilage control. These findings suggest several possibilities as with the results of the other stimulation groups previously mentioned. Specifically, our tension stimulation algorithm produced an effect that is similar to the superficial tangential zone aggrecan content and does not overproduce as when used in conjunction with compression.

\subsection{CONCLUSION, LIMITATIONS, AND FUTURE WORK}

Our hypothesis for this study was to create a functional tissue engineered construct with similar properties to the superficial zone using MSCs as a cell source, application of compression to increase matrix components, and application of tension for cellular and fiber alignment. Although the mechanical properties are inferior to native articular cartilage, the potential implications for the compression and tension loading regimen without offset shows improved properties overall. Additionally, the resultant aggregate modulus was only a magnitude lower and the tensile modulus $20 \mathrm{kPa}$ higher than reported values of repair tissue ${ }^{101}$. Improving the parameters of the culturing time period could possible enhance these properties. Various studies 
further support this theory - Angele et al. applied stimulation and retained cultures for 14 or 28 days before evaluation ${ }^{67}$, Lee et al. varied the pre-culture time period before application of stimulation ${ }^{102}$, and Terraciano et al. only precultured for 24 hours prior to stimulation regimens lasting 1,2 , or 3 weeks ${ }^{94}$. However, extension of time in culture needs to be carefully balanced as properties can plateau eventually and begin to decrease.

Our study design limited the amount of samples able to be tested. There is only one bioreactor chamber that can incorporate and mechanically stimulate 3 constructs at once. The extension of any culture past 2 weeks would create extremely slow production of samples to be evaluated. It may be necessary to replicate a second bioreactor or improve upon the design of the current setup to include loading of more samples for stimulation. The ability to stimulate more samples concurrently would allow for a greater number of samples tested, plus the ability to evaluate many different algorithms.

Additionally, the culturing of a static construct for the same time as our stimulated constructs was not feasible, due to excessive shrinkage in static culture of MSCs seeded in collagen I scaffolds, along with time constraints. By culturing non-stimulated constructs in the bioreactor, the grips would prevent lateral contraction along with an enhancement in media exchange through fluid flow providing better clues to further explain of the current data. Perfusion of samples in the chamber without stimulation was not included due to the limited capacity of the chamber, which would increase the study by 6 months or more to include perfused samples.

Western blot evaluated should have included collagen type II since it is a necessary component of cartilage tissue and would aid in identification of production of cartilage-like tissue. We did not evaluate for collagen type II due to cost and time constraints. We also only 
evaluated MSCs seeded on one type of scaffold material of collagen type I that is not native to articular cartilage. Perhaps using scaffolds of other compositions would achieve improved properties, particular collagen type II that would activate signals in the cells that are seen in native cartilage. Nerher et al. found different cell morphologies present when comparing canine chondrocytes seeded in either collagen type I or II sponges, illustrating scaffolds can effect cell morphology 35

The outcome of compression only with and without offset and tension only with offset, would aid in gaining a better knowledge of what algorithms best fit creating a STZ. Future work will include various extended culture in the chamber with various loading algorithms to include compression only with an offset and tension only with an offset, in addition to the previously tested loading parameters. Furthermore, use of other sponge scaffolds, such as collagen type II, which is native to articular cartilage, need to be evaluated with stimulation. Finally, inclusion of collagen type II analysis with western blot for verifying matrix components and fiber alignment analysis.

Overall, all stimulated constructs had some beneficial increase in content or structure taking into consideration all the results from the Day 0 and the different stimulated algorithms of this study. It seems that the combination of compression and tension with no offset produced the best characteristics out of all of our groups for a functional tissue engineered construct. It is possible that this chosen loading regimen created an environment that facilitated positive cellular response to many cues such as enhanced transport of nutrients and waste products and cellmatrix interactions creating increased matrix deposition and cell preferential arrangement that may ultimately lead to an STZ replacement with future cartilage tissue engineering exploration. 


\section{List of References}

1. Prevalence and most common causes of disability among adults--United States, 2005. MMWR Morb Mortal Wkly Rep 2009;58(16):421-6.

2. Glass GG. Osteoarthritis. Dis Mon 2006;52(9):343-62.

3. Mollenhauer JA. Perspectives on articular cartilage biology and osteoarthritis. Injury 2008;39 Suppl 1:S5-12.

4. Felson DT, Lawrence RC, Dieppe PA, Hirsch R, Helmick CG, Jordan JM, Kington RS, Lane NE, Nevitt MC, Zhang Y and others. Osteoarthritis: new insights. Part 1: the disease and its risk factors. Ann Intern Med 2000;133(8):635-46.

5. Simon TM, Jackson DW. Articular cartilage: injury pathways and treatment options. Sports Med Arthrosc 2006;14(3):146-54.

6. DeLee JC, Drez J. DeLee \& Drez's Orthopaedic Sports Medicine: Saunders; 2003.

7. Hunziker EB. Articular cartilage repair: basic science and clinical progress. A review of the current status and prospects. Osteoarthritis Cartilage 2002;10(6):432-63.

8. Buckwalter JA, Mankin HJ. Articular cartilage: tissue design and chondrocyte-matrix interactions. Instr Course Lect 1998;47:477-86.

9. Williams RJ, Peterson L, editors. Cartilage Repair Strategies. Totowa: Humana Press Inc.; 2007. $371 \mathrm{p}$.

10. Mow VC, Guo XE. Mechano-electrochemical Properties of Articular Cartilage: Their Inhomogeneities and Anisotropies. Annu Rev Biomed Eng 2002;4:175-209.

11. Cremer MA, Rosloniec EF, Kang AH. The cartilage collagens: a review of their structure, organization, and role in the pathogenesis of experimental arthritis in animals and in human rheumatic disease. J Mol Med 1998;76(3-4):275-88.

12. Gentili C, Cancedda R. Cartilage and bone extracellular matrix. Curr Pharm Des 2009;15(12):1334-48.

13. Kumar P, Oka M, Toguchida J, Kobayashi M, Uchida E, Nakamura T, Tanaka K. Role of uppermost superficial surface layer of articular cartilage in the lubrication mechanism of joints. J Anat 2001;199(Pt 3):241-50.

14. Oatis CA. Kinesiology: The Mechanics and Pathomechanics of Human Movement. Philadelphia: Lippincott Williams \& Wilkins; 2004.

15. Athanasiou KA, Rosenwasser MP, Buckwalter JA, Malinin TI, Mow VC. Interspecies comparisons of in situ intrinsic mechanical properties of distal femoral cartilage. J Orthop Res 1991;9(3):330-40.

16. Maroudas A. Physicochemical properties of cartilage in the light of ion exchange theory. Biophys J 1968;8(5):575-95.

17. Maroudas A, Bullough P. Permeability of articular cartilage. Nature 1968;219(5160):1260-1.

18. Maroudas A, Bullough P, Swanson SA, Freeman MA. The permeability of articular cartilage. J Bone Joint Surg Br 1968;50(1):166-77.

19. Lai WM, Mow VC. Drag-induced compression of articular cartilage during a permeation experiment. Biorheology 1980;17(1-2):111-23. 
20. Mansour JM, Mow VC. The permeability of articular cartilage under compressive strain and at high pressures. J Bone Joint Surg Am 1976;58(4):509-16.

21. Hayes WC, Bodine AJ. Flow-independent viscoelastic properties of articular cartilage matrix. J Biomech 1978;11(8-9):407-19.

22. Roth V, Mow VC. The intrinsic tensile behavior of the matrix of bovine articular cartilage and its variation with age. J Bone Joint Surg Am 1980;62(7):1102-17.

23. Schmidt MB, Mow VC, Chun LE, Eyre DR. Effects of proteoglycan extraction on the tensile behavior of articular cartilage. J Orthop Res 1990;8(3):353-63.

24. Buckwalter JA, Mankin HJ. Articular cartilage: degeneration and osteoarthritis, repair, regeneration, and transplantation. Instr Course Lect 1998;47:487-504.

25. Linn FC. Lubrication of animal joints. I. The arthrotripsometer. J Bone Joint Surg Am 1967;49(6):1079-98.

26. Redman SN, Oldfield SF, Archer CW. Current strategies for articular cartilage repair. Eur Cell Mater 2005;9:23-32; discussion 23-32.

27. Vinatier C, Mrugala D, Jorgensen C, Guicheux J, Noel D. Cartilage engineering: a crucial combination of cells, biomaterials and biofactors. Trends Biotechnol 2009.

28. van Osch GJ, Brittberg M, Dennis JE, Bastiaansen-Jenniskens YM, Erben RG, Konttinen YT, Luyten FP. Cartilage repair: past and future. J Cell Mol Med 2009.

29. Hunter CJ, Mouw JK, Levenston ME. Dynamic compression of chondrocyte-seeded fibrin gels: effects on matrix accumulation and mechanical stiffness. Osteoarthritis Cartilage 2004;12(2):117-30.

30. Chu CR, Convery FR, Akeson WH, Meyers M, Amiel D. Articular cartilage transplantation. Clinical results in the knee. Clin Orthop Relat Res 1999(360):159-68.

31. Brighton CT, Shadle CA, Jimenez SA, Irwin JT, Lane JM, Lipton M. Articular cartilage preservation and storage. I. Application of tissue culture techniques to the storage of viable articular cartilage. Arthritis Rheum 1979;22(10):1093-101.

32. Luyten FP, Hascall VC, Nissley SP, Morales TI, Reddi AH. Insulin-like growth factors maintain steady-state metabolism of proteoglycans in bovine articular cartilage explants. Arch Biochem Biophys 1988;267(2):416-25.

33. Strehl R, Tallheden T, Sjogren-Jansson E, Minuth WW, Lindahl A. Long-term maintenance of human articular cartilage in culture for biomaterial testing. Biomaterials 2005;26(22):4540-9.

34. Ficklin T, Thomas G, Barthel JC, Asanbaeva A, Thonar EJ, Masuda K, Chen AC, Sah RL, Davol A, Klisch SM. Articular cartilage mechanical and biochemical property relations before and after in vitro growth. J Biomech 2007;40(16):3607-14.

35. Nehrer S, Breinan HA, Ramappa A, Shortkroff S, Young G, Minas T, Sledge CB, Yannas IV, Spector M. Canine chondrocytes seeded in type I and type II collagen implants investigated in vitro. J Biomed Mater Res 1997;38(2):95-104.

36. Lee HJ, Yu C, Chansakul T, Hwang NS, Varghese S, Yu SM, Elisseeff JH. Enhanced chondrogenesis of mesenchymal stem cells in collagen mimetic peptide-mediated microenvironment. Tissue Eng Part A 2008;14(11):1843-51.

37. Huang AH, Yeger-McKeever M, Stein A, Mauck RL. Tensile properties of engineered cartilage formed from chondrocyte- and MSC-laden hydrogels. Osteoarthritis Cartilage 2008;16(9):1074-82. 
38. Huang CY, Reuben PM, D'Ippolito G, Schiller PC, Cheung HS. Chondrogenesis of human bone marrow-derived mesenchymal stem cells in agarose culture. Anat Rec A Discov Mol Cell Evol Biol 2004;278(1):428-36.

39. Lee CR, Grodzinsky AJ, Hsu HP, Spector M. Effects of a cultured autologous chondrocyte-seeded type II collagen scaffold on the healing of a chondral defect in a canine model. J Orthop Res 2003;21(2):272-81.

40. Mauck RL, Yuan X, Tuan RS. Chondrogenic differentiation and functional maturation of bovine mesenchymal stem cells in long-term agarose culture. Osteoarthritis Cartilage 2006;14(2):179-89.

41. Jones IL, Klamfeldt A, Sandstrom T. The effect of continuous mechanical pressure upon the turnover of articular cartilage proteoglycans in vitro. Clin Orthop Relat Res 1982(165):283-9.

42. Palmoski MJ, Brandt KD. Effects of static and cyclic compressive loading on articular cartilage plugs in vitro. Arthritis Rheum 1984;27(6):675-81.

43. Sah RL, Kim YJ, Doong JY, Grodzinsky AJ, Plaas AH, Sandy JD. Biosynthetic response of cartilage explants to dynamic compression. J Orthop Res 1989;7(5):619-36.

44. Waldman SD, Couto DC, Grynpas MD, Pilliar RM, Kandel RA. A single application of cyclic loading can accelerate matrix deposition and enhance the properties of tissueengineered cartilage. Osteoarthritis Cartilage 2006;14(4):323-30.

45. Lee CR, Grodzinsky AJ, Spector M. Biosynthetic response of passaged chondrocytes in a type II collagen scaffold to mechanical compression. J Biomed Mater Res 2003;64A(3):560-9.

46. Demarteau O, Wendt D, Braccini A, Jakob M, Schafer D, Heberer M, Martin I. Dynamic compression of cartilage constructs engineered from expanded human articular chondrocytes. Biochem Biophys Res Commun 2003;310(2):580-8.

47. Pelaez D, Huang CY, Cheung HS. Cyclic Compression Maintains Viability and Induces Chondrogenesis of Human Mesenchymal Stem Cells in Fibrin Gel Scaffolds. Stem Cells Dev 2008 .

48. Huang CY, Hagar KL, Frost LE, Sun Y, Cheung HS. Effects of cyclic compressive loading on chondrogenesis of rabbit bone-marrow derived mesenchymal stem cells. Stem Cells 2004;22(3):313-23.

49. Ng KW, Mauck RL, Statman LY, Lin EY, Ateshian GA, Hung CT. Dynamic deformational loading results in selective application of mechanical stimulation in a layered, tissue-engineered cartilage construct. Biorheology 2006;43(3-4):497-507.

50. Lima EG, Bian L, Mauck RL, Byers BA, Tuan RS, Ateshian GA, Hung CT. The effect of applied compressive loading on tissue-engineered cartilage constructs cultured with TGFbeta3. Conf Proc IEEE Eng Med Biol Soc 2006;1:779-82.

51. Toyoda T, Saito S, Inokuchi S, Yabe Y. The effects of tensile load on the metabolism of cultured chondrocytes. Clin Orthop 1999(359):221-8.

52. Honda K, Ohno S, Tanimoto K, Ijuin C, Tanaka N, Doi T, Kato Y, Tanne K. The effects of high magnitude cyclic tensile load on cartilage matrix metabolism in cultured chondrocytes. Eur J Cell Biol 2000;79(9):601-9.

53. Fukuda K, Asada S, Kumano F, Saitoh M, Otani K, Tanaka S. Cyclic tensile stretch on bovine articular chondrocytes inhibits protein kinase $\mathrm{C}$ activity. J Lab Clin Med 1997;130(2):209-15. 
54. De Witt MT, Handley CJ, Oakes BW, Lowther DA. In vitro response of chondrocytes to mechanical loading. The effect of short term mechanical tension. Connect Tissue Res 1984;12(2):97-109.

55. Yang X, Vezeridis PS, Nicholas B, Crisco JJ, Moore DC, Chen Q. Differential expression of type $\mathrm{X}$ collagen in a mechanically active 3-D chondrocyte culture system: a quantitative study. J Orthop Surg Res 2006;1:15.

56. Liu M, Montazeri S, Jedlovsky T, Van Wert R, Zhang J, Li RK, Yan J. Bio-stretch, a computerized cell strain apparatus for three-dimensional organotypic cultures. In Vitro Cell Dev Biol Anim 1999;35(2):87-93.

57. Vanderploeg EJ, Imler SM, Brodkin KR, Garcia AJ, Levenston ME. Oscillatory tension differentially modulates matrix metabolism and cytoskeletal organization in chondrocytes and fibrochondrocytes. J Biomech 2004;37(12):1941-52.

58. McMahon LA, Campbell VA, Prendergast PJ. Involvement of stretch-activated ion channels in strain-regulated glycosaminoglycan synthesis in mesenchymal stem cellseeded 3D scaffolds. J Biomech 2008;41(9):2055-9.

59. Nirmalanandhan VS, Dressler MR, Shearn JT, Juncosa-Melvin N, Rao M, Gooch C, Bradica G, Butler DL. Mechanical stimulation of tissue engineered tendon constructs: effect of scaffold materials. J Biomech Eng 2007;129(6):919-23.

60. Chokalingam K, Juncosa-Melvin N, Hunter SA, Gooch C, Frede C, Bradica G, Wenstrup R, Butler DL. Tensile Stimulation Of Murine Stem Cell-Collagen Sponge Constructs Increases Collagen Type I Gene Expression And Linear Stiffness. Tissue Eng Part A 2009.

61. Abousleiman RI, Reyes Y, McFetridge P, Sikavitsas V. Tendon tissue engineering using cell-seeded umbilical veins cultured in a mechanical stimulator. Tissue Eng Part A 2009;15(4):787-95.

62. Setton LA, Zhu W, Mow VC. The biphasic poroviscoelastic behavior of articular cartilage: role of the surface zone in governing the compressive behavior. J Biomech 1993;26(4-5):581-92.

63. Pountos I, Jones E, Tzioupis C, McGonagle D, Giannoudis PV. Growing bone and cartilage: THE ROLE OF MESENCHYMAL STEM CELLS. J Bone Joint Surg Br 2006;88-B(4):421-426.

64. Worster AA, Brower-Toland BD, Fortier LA, Bent SJ, Williams J, Nixon AJ. Chondrocytic differentiation of mesenchymal stem cells sequentially exposed to transforming growth factor-beta1 in monolayer and insulin-like growth factor-I in a three-dimensional matrix. J Orthop Res 2001;19(4):738-49.

65. Caterson EJ, Nesti LJ, Li WJ, Danielson KG, Albert TJ, Vaccaro AR, Tuan RS. Threedimensional cartilage formation by bone marrow-derived cells seeded in polylactide/alginate amalgam. J Biomed Mater Res 2001;57(3):394-403.

66. Li WJ, Tuli R, Huang X, Laquerriere P, Tuan RS. Multilineage differentiation of human mesenchymal stem cells in a three-dimensional nanofibrous scaffold. Biomaterials 2005;26(25):5158-66.

67. Angele P, Yoo JU, Smith C, Mansour J, Jepsen KJ, Nerlich M, Johnstone B. Cyclic hydrostatic pressure enhances the chondrogenic phenotype of human mesenchymal progenitor cells differentiated in vitro. J Orthop Res 2003;21(3):451-7. 
68. Angele P, Schumann D, Angele M, Kinner B, Englert C, Hente R, Fuchtmeier B, Nerlich M, Neumann C, Kujat R. Cyclic, mechanical compression enhances chondrogenesis of mesenchymal progenitor cells in tissue engineering scaffolds. Biorheology 2004;41(34):335-46.

69. Waldman SD, Spiteri CG, Grynpas MD, Pilliar RM, Kandel RA. Long-term intermittent compressive stimulation improves the composition and mechanical properties of tissueengineered cartilage. Tissue Eng 2004;10(9-10):1323-31.

70. The Public Fountains of the City of Dijon by Henry Darcy (1856). Dubuque; 2004.

71. Wartella KA, Wayne JS. Permeability of Mesenchymal Stem Cell Laden Cartilage Constructs. 2008; San Francisco, CA.

72. Maroudas A. Biophysical chemistry of cartilaginous tissues with special reference to solute and fluid transport. Biorheology 1975;12(3-4):233-48.

73. Wartella KA, Wayne JS. Bioreactor for biaxial mechanical stimulation to tissue engineered constructs. J Biomech Eng 2009;131(4):044501.

74. Houtchens GR, Foster MD, Desai TA, Morgan EF, Wong JY. Combined effects of microtopography and cyclic strain on vascular smooth muscle cell orientation. J Biomech 2008;41(4):762-9.

75. Mow VC, Good PM, Gardner TR. A new method to determine the tensile properties of articular cartilage using the indentation test. 2000.

76. Mak AF, Lai WM, Mow VC. Biphasic indentation of articular cartilage--I. Theoretical analysis. J Biomech 1987;20(7):703-14.

77. Sumer EU, Madsen SH, Sondergaard B-C, Christiansen C, Qvist P, Karsdal MA. Aggrecan Constists of Two Distinct Pools: One High-Molecular-Weight, Aggrecanase Degradable Reversible Pool, As Well As An Irreversible, MMP-Susceptible LowMolecular-Weight Pool. Osteoarthritis and Cartilage 2007;15.

78. Buschmann MD, Gluzband YA, Grodzinsky AJ, Hunziker EB. Mechanical compression modulates matrix biosynthesis in chondrocyte/agarose culture. J Cell Sci 1995;108 (Pt 4):1497-508.

79. Connelly JT, Vanderploeg EJ, Mouw JK, Wilson CG, Levenston ME. Tensile loading modulates bone marrow stromal cell differentiation and the development of engineered fibrocartilage constructs. Tissue Eng Part A;16(6):1913-23.

80. Li Z, Kupcsik L, Yao SJ, Alini M, Stoddart MJ. Chondrogenesis of Human Bone Marrow Mesenchymal Stem Cells in Fibrin-Polyurethane Composites. Tissue Eng Part A 2008.

81. Emin N, Koc A, Durkut S, Elcin AE, Elcin YM. Engineering of rat articular cartilage on porous sponges: effects of tgf-beta 1 and microgravity bioreactor culture. Artif Cells Blood Substit Immobil Biotechnol 2008;36(2):123-37.

82. Stok KS, Lisignoli G, Cristino S, Facchini A, Muller R. Mechano-functional assessment of human mesenchymal stem cells grown in three-dimensional hyaluronan-based scaffolds for cartilage tissue engineering. J Biomed Mater Res A;93(1):37-45.

83. Wise JK, Yarin AL, Megaridis CM, Cho M. Chondrogenic differentiation of human mesenchymal stem cells on oriented nanofibrous scaffolds: engineering the superficial zone of articular cartilage. Tissue Eng Part A 2009;15(4):913-21.

84. Valmikinathan CM, Tian J, Wang J, Yu X. Novel nanofibrous spiral scaffolds for neural tissue engineering. J Neural Eng 2008;5(4):422-32. 
85. Woo SL, Mow VC, Lai WM. Biomechanical Properties of Articular Cartilage. In: Skalak R, Chien S, editors. Handbook of Bioengineering. New York: McGraw-Hill; 1986.

86. Huang CY, Stankiewicz A, Ateshian GA, Mow VC. Anisotropy, inhomogeneity, and tension-compression nonlinearity of human glenohumeral cartilage in finite deformation. J Biomech 2005;38(4):799-809.

87. Hori RY, Mockros LF. Indentation tests of human articular cartilage. J Biomech 1976;9(4):259-68.

88. Korhonen RK, Laasanen MS, Toyras J, Rieppo J, Hirvonen J, Helminen HJ, Jurvelin JS. Comparison of the equilibrium response of articular cartilage in unconfined compression, confined compression and indentation. J Biomech 2002;35(7):903-9.

89. Hunziker EB, Quinn TM, Hauselmann HJ. Quantitative structural organization of normal adult human articular cartilage. Osteoarthritis Cartilage 2002;10(7):564-72.

90. Poole AR, Kojima T, Yasuda T, Mwale F, Kobayashi M, Laverty S. Composition and structure of articular cartilage: a template for tissue repair. Clin Orthop Relat Res 2001(391 Suppl):S26-33.

91. Holledge MM, Millward-Sadler SJ, Nuki G, Salter DM. Mechanical regulation of proteoglycan synthesis in normal and osteoarthritic human articular chondrocytes--roles for alpha5 and alphaVbeta5 integrins. Biorheology 2008;45(3-4):275-88.

92. Nguyen TD, Liang R, Woo SL, Burton SD, Wu C, Almarza A, Sacks MS, Abramowitch $\mathrm{S}$. Effects of cell seeding and cyclic stretch on the fiber remodeling in an extracellular matrix-derived bioscaffold. Tissue Eng Part A 2009;15(4):957-63.

93. Mauck RL, Byers BA, Yuan X, Tuan RS. Regulation of cartilaginous ECM gene transcription by chondrocytes and MSCs in 3D culture in response to dynamic loading. Biomech Model Mechanobiol 2007;6(1-2):113-25.

94. Terraciano V, Hwang N, Moroni L, Park HB, Zhang Z, Mizrahi J, Seliktar D, Elisseeff J. Differential response of adult and embryonic mesenchymal progenitor cells to mechanical compression in hydrogels. Stem Cells 2007;25(11):2730-8.

95. Henshaw DR, Attia E, Bhargava M, Hannafin JA. Canine ACL fibroblast integrin expression and cell alignment in response to cyclic tensile strain in three-dimensional collagen gels. J Orthop Res 2006;24(3):481-90.

96. Au-Yeung KL, Sze KY, Sham MH, Chan BP. Development of a micromanipulator-based loading device for mechanoregulation study of human mesenchymal stem cells in threedimensional collagen constructs. Tissue Eng Part C Methods;16(1):93-107.

97. Hunziker EB, Kapfinger E. Removal of proteoglycans from the surface of defects in articular cartilage transiently enhances coverage by repair cells. J Bone Joint Surg Br 1998;80(1):144-50.

98. Davisson T, Kunig S, Chen A, Sah R, Ratcliffe A. Static and dynamic compression modulate matrix metabolism in tissue engineered cartilage. J Orthop Res 2002;20(4):8428 .

99. Seidel JO, Pei M, Gray ML, Langer R, Freed LE, Vunjak-Novakovic G. Long-term culture of tissue engineered cartilage in a perfused chamber with mechanical stimulation. Biorheology 2004;41(3-4):445-58.

100. Zeichen J, van Griensven M, Bosch U. The proliferative response of isolated human tendon fibroblasts to cyclic biaxial mechanical strain. Am J Sports Med 2000;28(6):88892. 
101. Cohen B, Gardner T, Ateshian GA. The influence of transverse isotropy on cartilage indentation behavior: a study of the human humeral head. 1993.

102. Lee CR, Breinan HA, Nehrer S, Spector M. Articular cartilage chondrocytes in type I and type II collagen-GAG matrices exhibit contractile behavior in vitro. Tissue Eng 2000;6(5):555-65. 


\section{APPENDIX A}
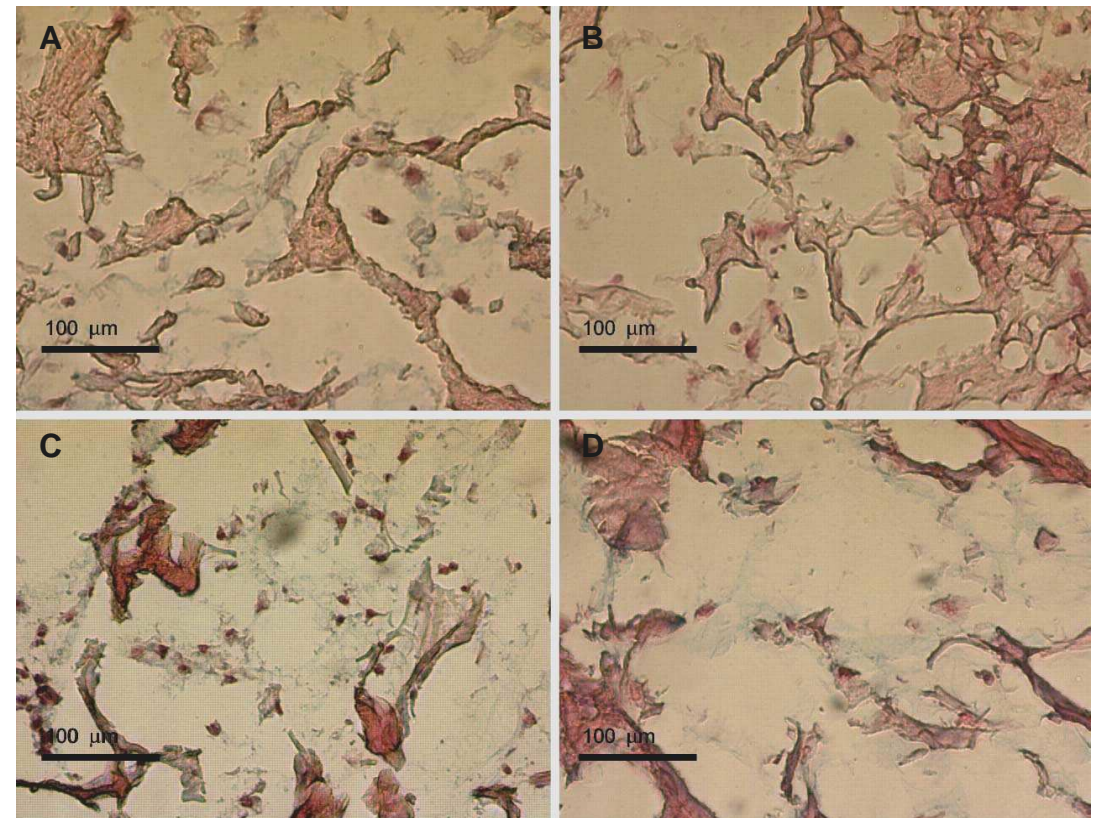

Figure A.1 Construct 155A (A and B) and Construct 186D (C and D), Day 0 with no mechanical stimulation. Shown are edge sections with black arrows indicating the exterior surface (A and C) and a plane parallel to the surface (B and D). (scale bar is $100 \square \mathrm{m}$ ).
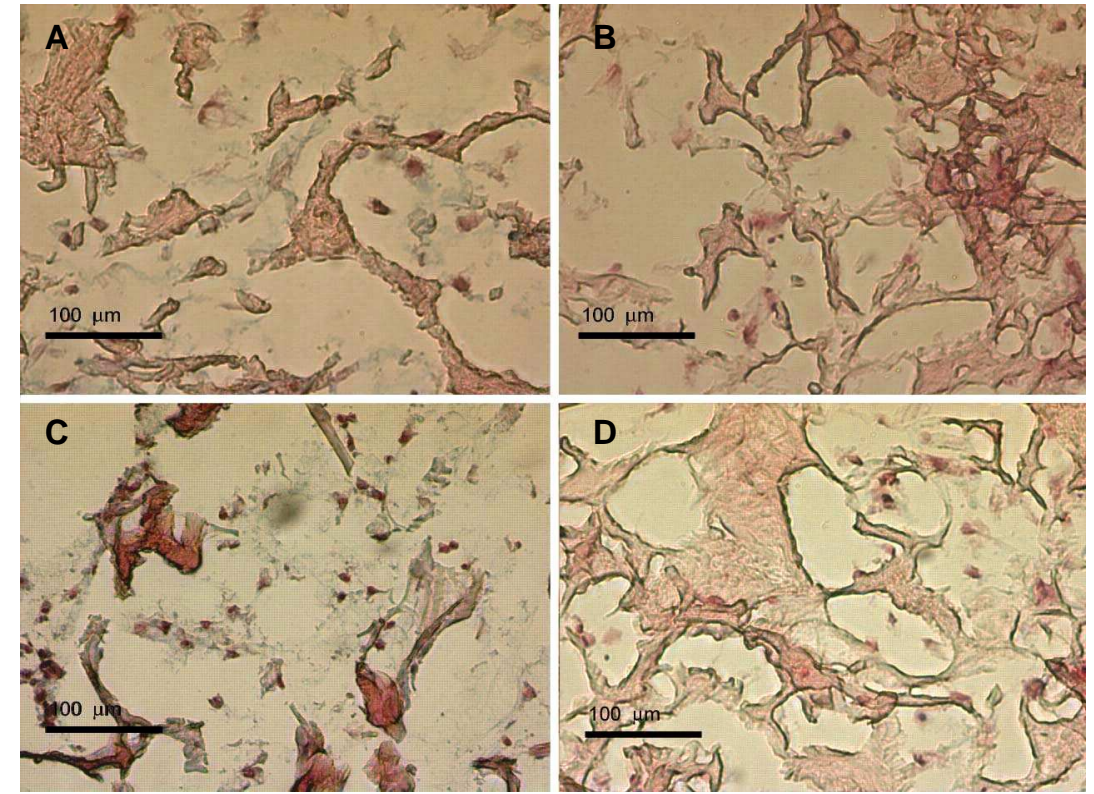

Figure A.2 Construct 155A (A and B) and Construct 186D (C and D), Day 0 with no mechanical stimulation. Shown are edge sections with black arrows indicating the exterior surface (A and C) and a plane parallel to the surface (B and D). (scale bar is $100 \mu \mathrm{m}$ ). 

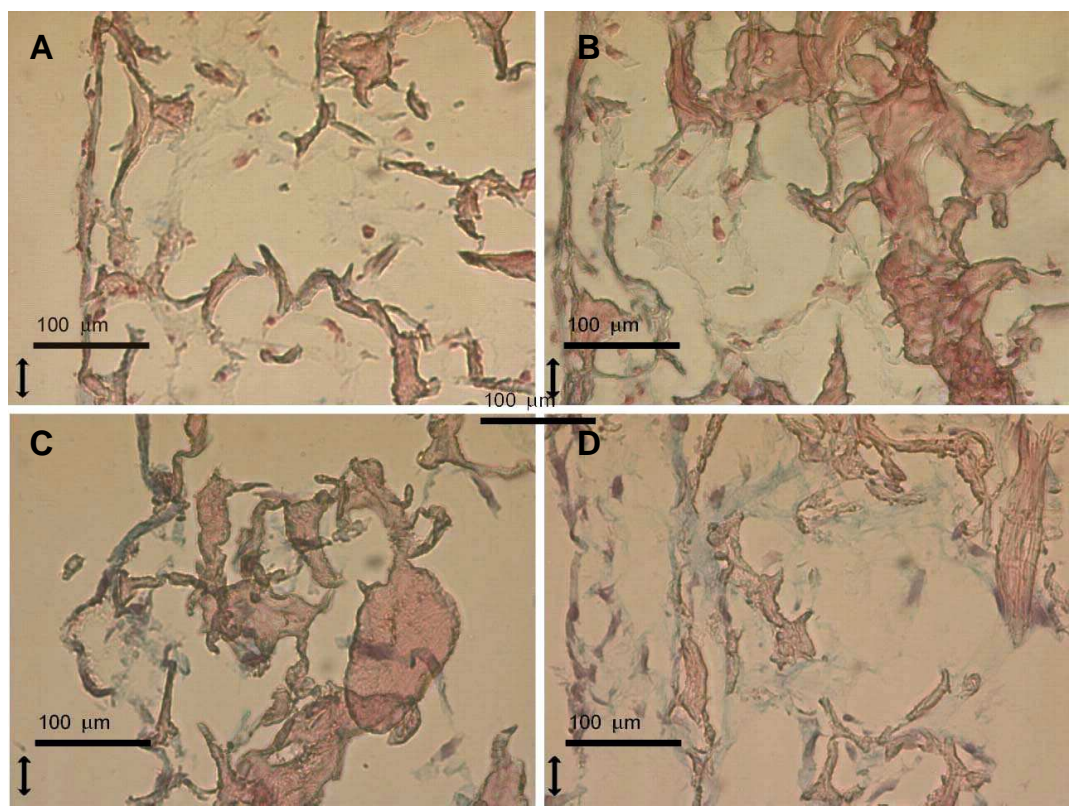

Figure A.3 Construct 163A (A and B) and Construct 209A (C and D) with both compression and tension without offset. Shown are edge sections, with black arrows indicating the exterior surface (A and C), and interior plane sections (B and D). (scale bar is $100 \square \mathrm{m}$ ).
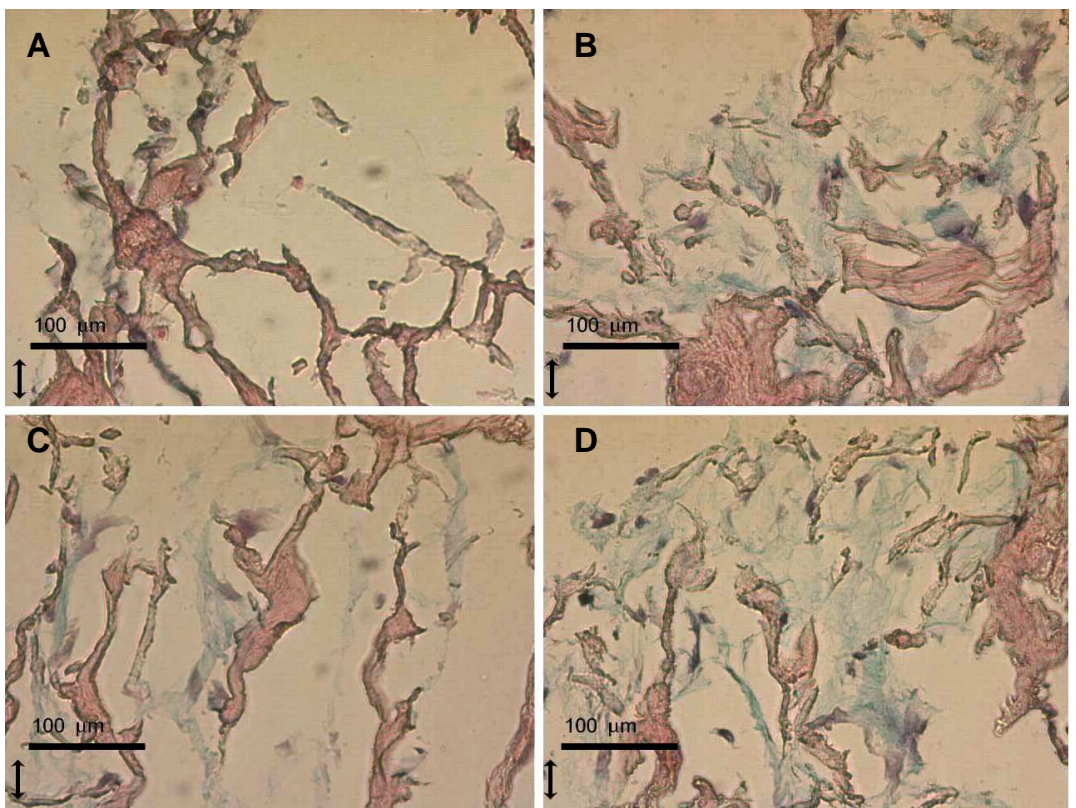

Figure A.4 Construct 163A (A and B) and Construct 209A (C and D) with both compression and tension without offset. Shown are edge sections, with black arrows indicating the exterior surface (A and C), and interior plane sections (B and D). (scale bar is $100 \mu \mathrm{m}$ ). 

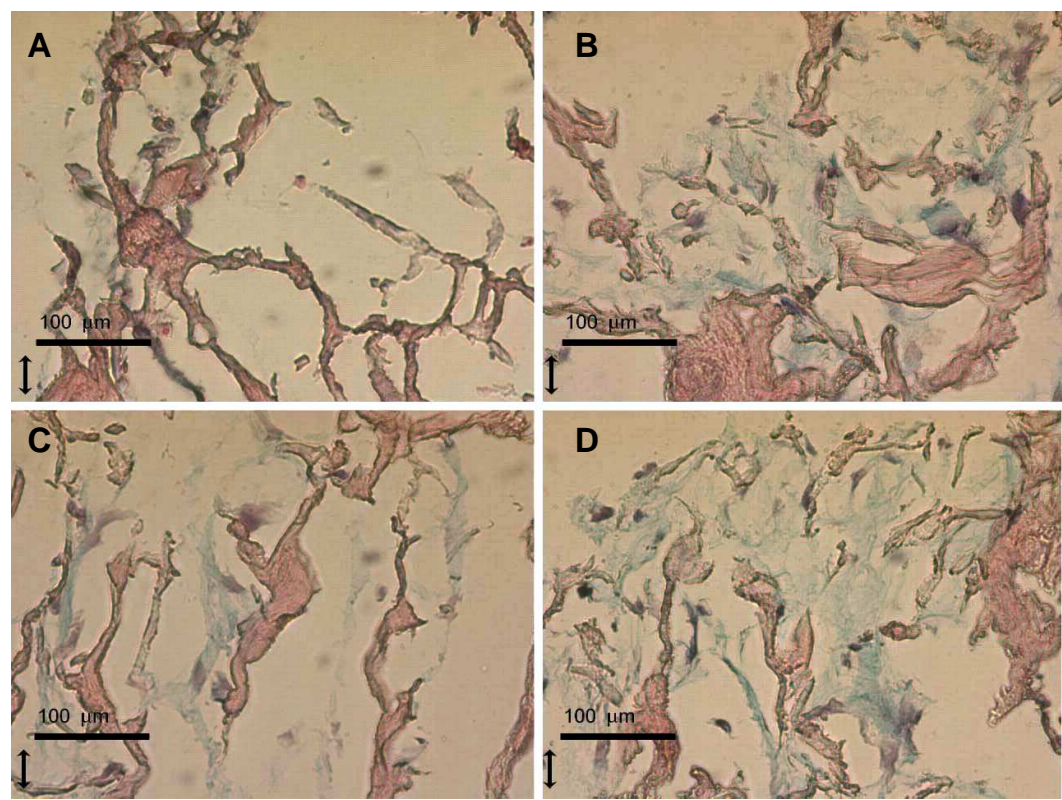

Figure A.5 Construct 186B (A and B) and Construct 188B (C and D) with both compression and tension with an offset, illustrating edge sections, with black arrows indicating the exterior surface $(\mathrm{A}$ and $\mathrm{C})$ and interior plane sections (B and D). (scale bar is $100 \square \mathrm{m}$ ).

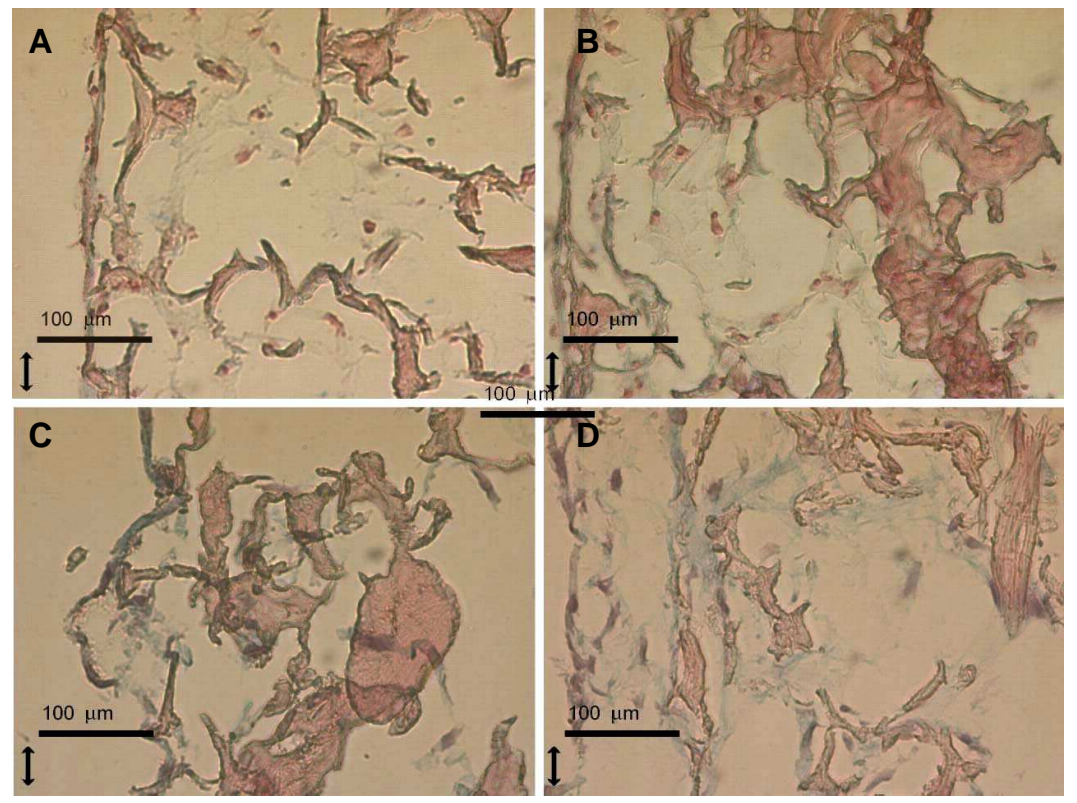

Figure A.6 Construct 186B (A and B) and Construct 188B (C and D) with both compression and tension with an offset, illustrating edge sections, with black arrows indicating the exterior surface (A and $\mathrm{C})$ and interior plane sections (B and D). (scale bar is $100 \mu \mathrm{m}$ ). 

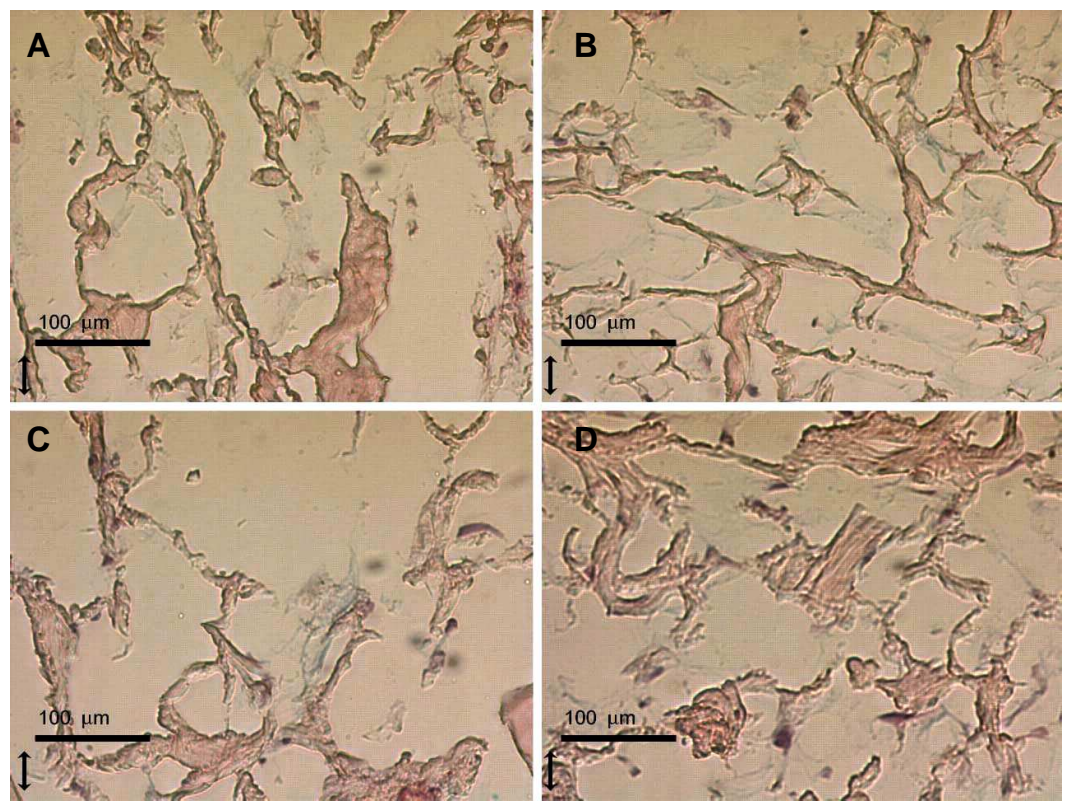

Figure A.7 Construct 197B (A and B) and Construct 199A (C and D) with both compression and tension with an offset, illustrating edge sections, with black arrows indicating the exterior surface (A and $\mathrm{C}$ ) and interior plane sections (B and D). (scale bar is $100 \square \mathrm{m}$ ).

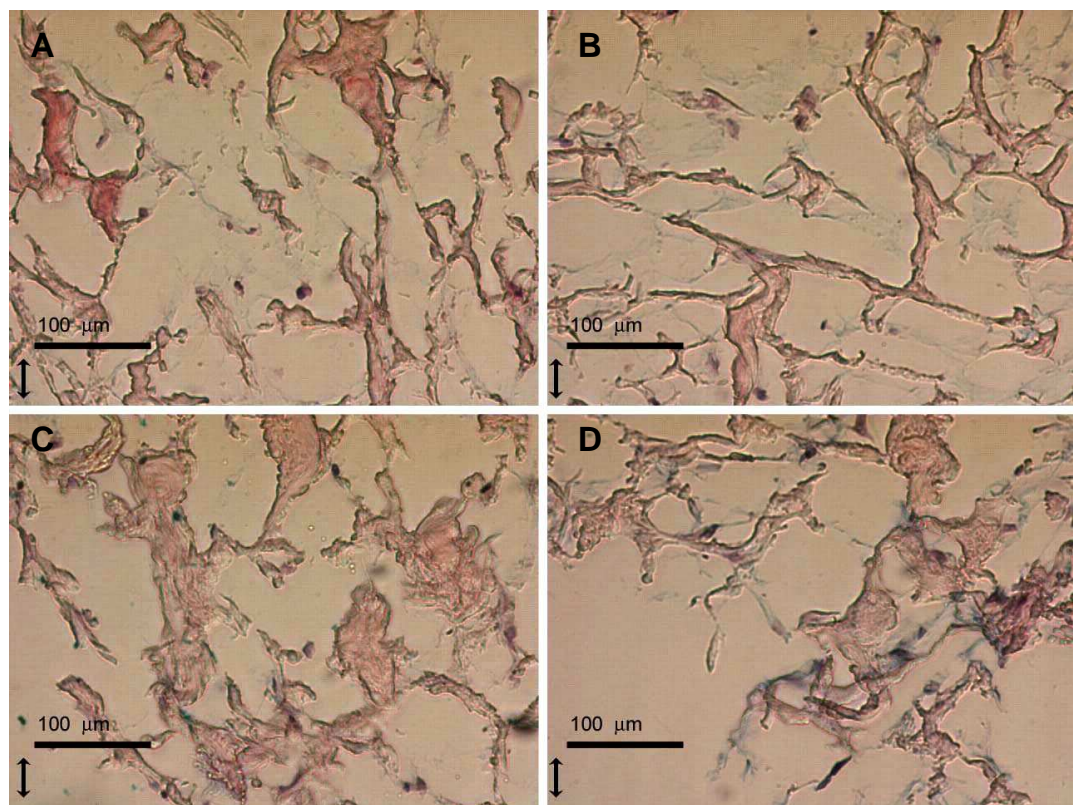

Figure A.8 Construct 197B (A and B) and Construct 199A (C and D) with both compression and tension with an offset, illustrating edge sections, with black arrows indicating the exterior surface (A and $\mathrm{C}$ ) and interior plane sections (B and D). (scale bar is $100 \square \mathrm{m}$ ). 

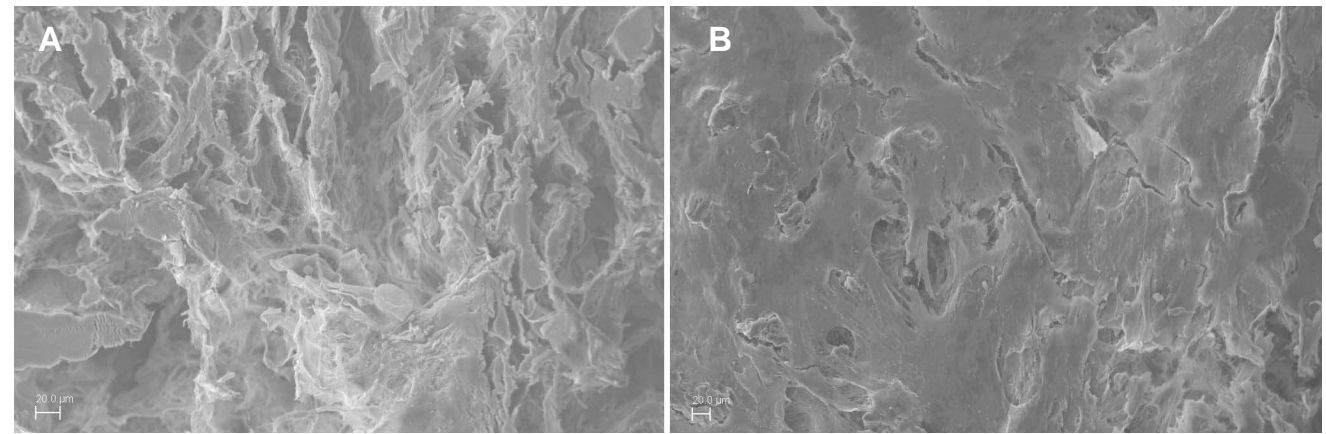

Figure A.9 Construct 212A with no stimulation, illustrating an (A) internal edge section view and (B) internal parallel to interior plane section (scale bar is $20 \mu \mathrm{m}$ ).
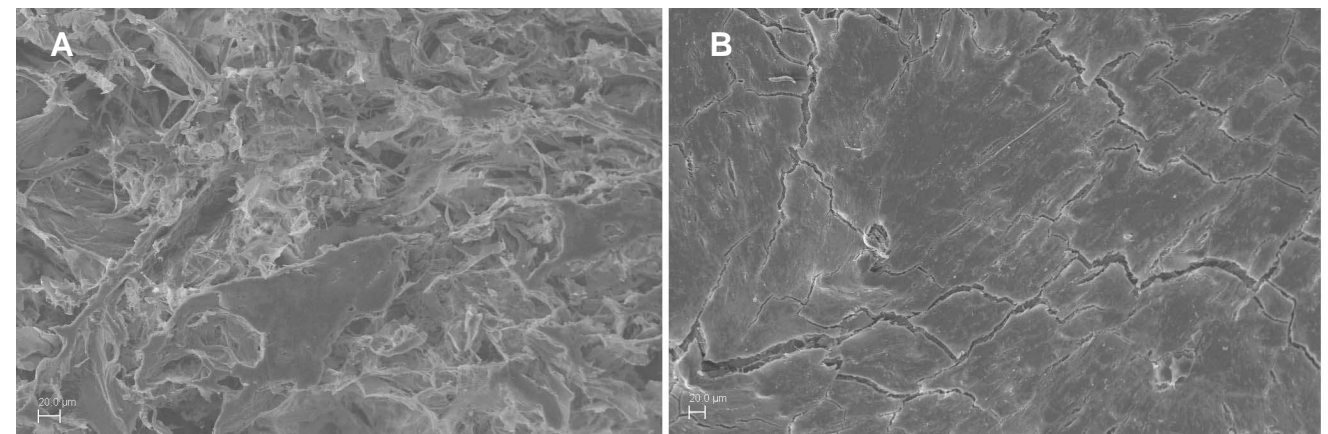

Figure A.10 Construct 212B with no stimulation, illustrating an (A) internal edge section view and (B) internal parallel to interior plane section (scale bar is $20 \mu \mathrm{m}$ ).
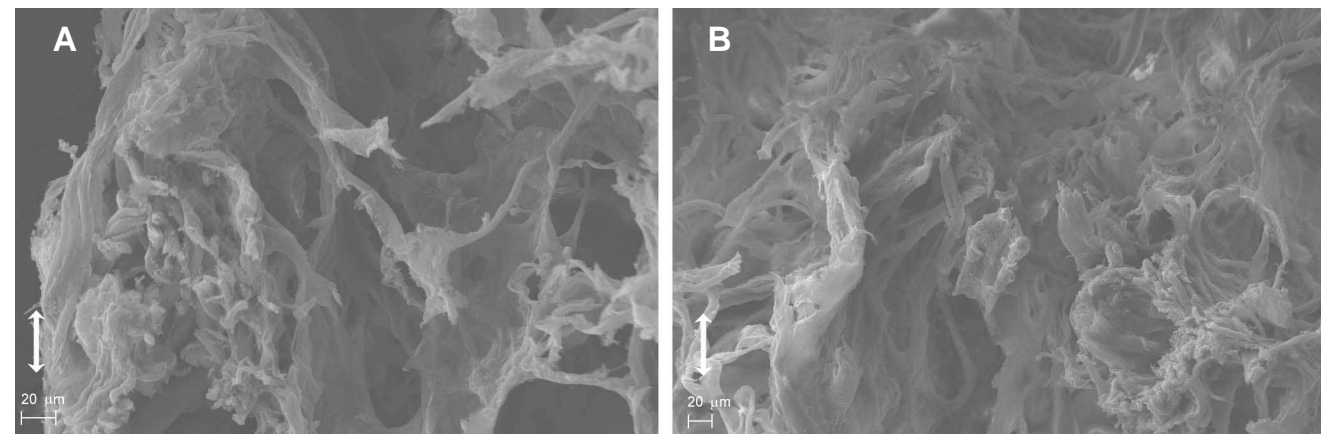

Figure A.11 Construct 166C with both compression and tension without offset, illustrating an (A) internal edge section view and (B) internal parallel to interior plane section, white double-headed arrows indicate direction of tension application (scale bar is $20 \mu \mathrm{m}$ ). 

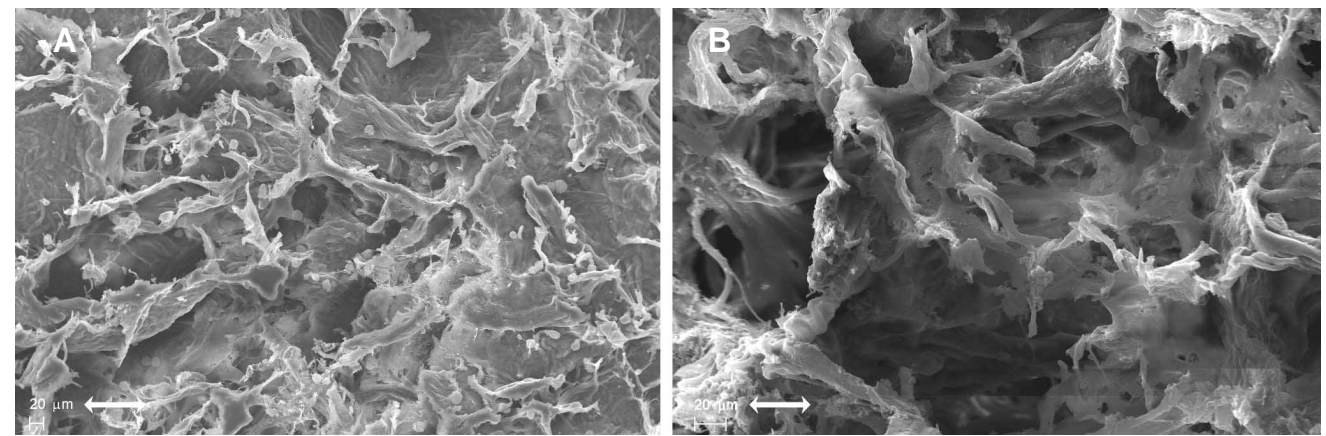

Figure A.12 Construct 170A with both compression and tension without offset, illustrating an (A) internal edge section view and (B) internal parallel to interior plane section, white double-headed arrows indicate direction of tension application (scale bar is $20 \mu \mathrm{m}$ ).
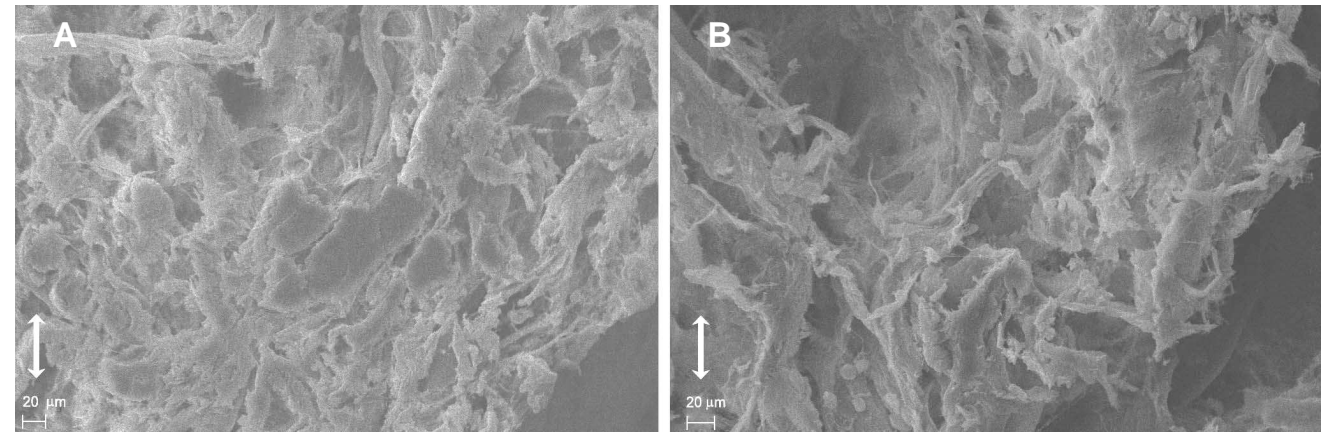

Figure A.13 Construct 186B with both compression and tension with offset, illustrating an (A) internal edge section view and (B) internal parallel to interior plane section, white double-headed arrows indicate direction of tension application (scale bar is $20 \mu \mathrm{m}$ ).
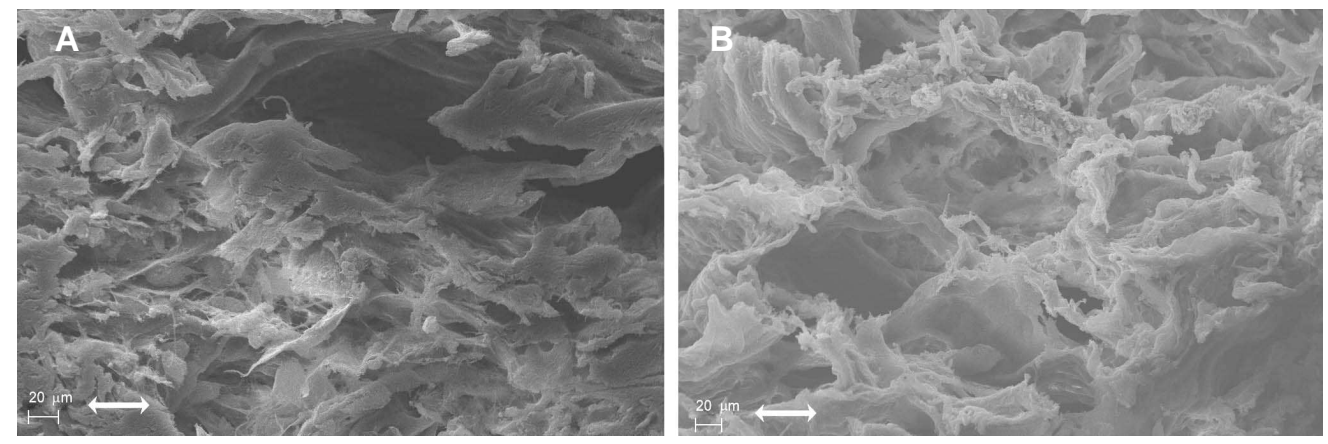

Figure A.14 Construct 188B with both compression and tension with offset, illustrating an (A) internal edge section view and (B) internal parallel to interior plane section, white double-headed arrows indicate direction of tension application (scale bar is $20 \mu \mathrm{m}$ ). 

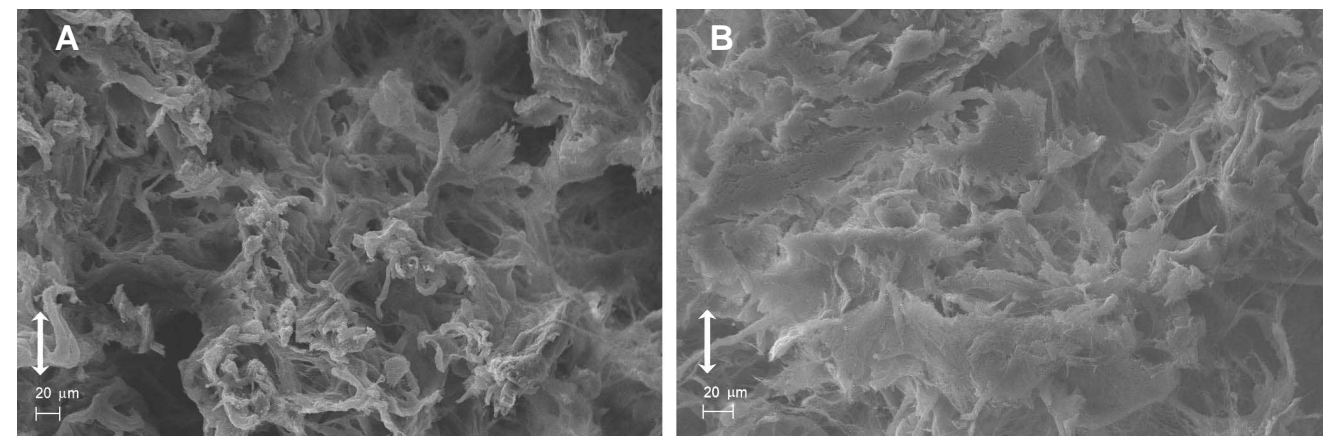

Figure A.15 Construct 198B with tension only, illustrating an (A) internal edge section view and (B) internal parallel to interior plane section, white double-headed arrows indicate direction of tension application (scale bar is $20 \mu \mathrm{m})$.
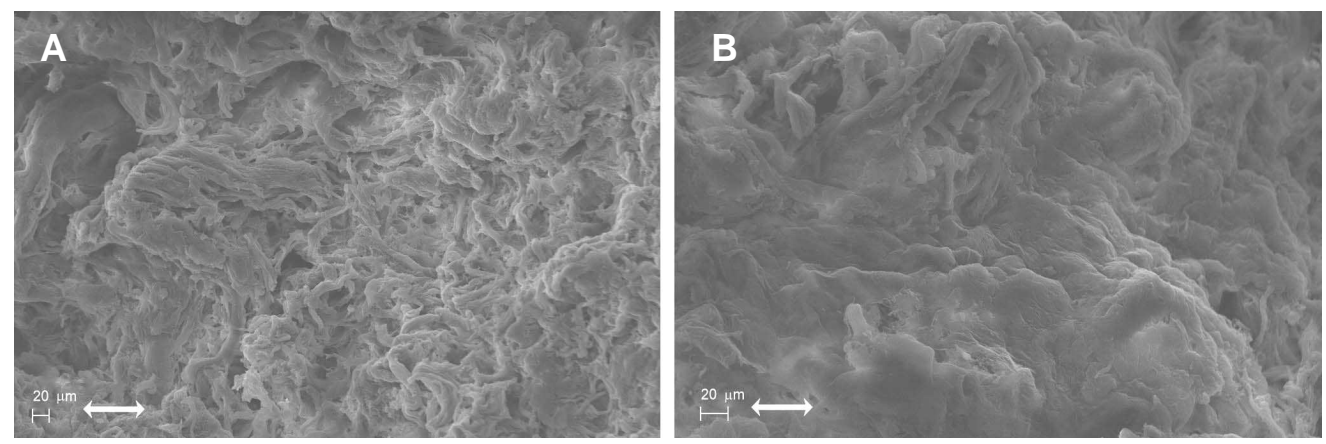

Figure A.16 Construct 199A with tension only, illustrating an (A) internal edge section view and (B) internal parallel to interior plane section, white double-headed arrows indicate direction of tension application (scale bar is $20 \mu \mathrm{m})$. 
Vita

Karin Anastasia Wartella was born on September 25, 1974, in Wilkes-Barre Pennsylvania. She graduated from Dallas High School, Dallas, Pennsylvania in 1992. In 1997 she graduated cum laude with her Bachelor of Science in Biology form King's College, Wilkes-Barre, Pennsylvania. She worked in pharmaceutical research at Covance Laboratories, Vienna, Virginia from 1999 to 2002. She received a Master of Science in Biomedical Engineering from Virginia Commonwealth University in 2005. 